
Pal Seanor - D.B.C. $-4 b^{\prime}$ 



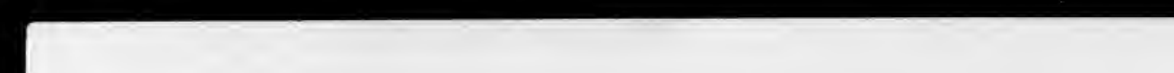




\section{BEGINNER'S GRAMMAR OF}

THE GREEK NEW TESTAMENT

WILliaM HERSEY DAVIS, M.A., TH.D. 


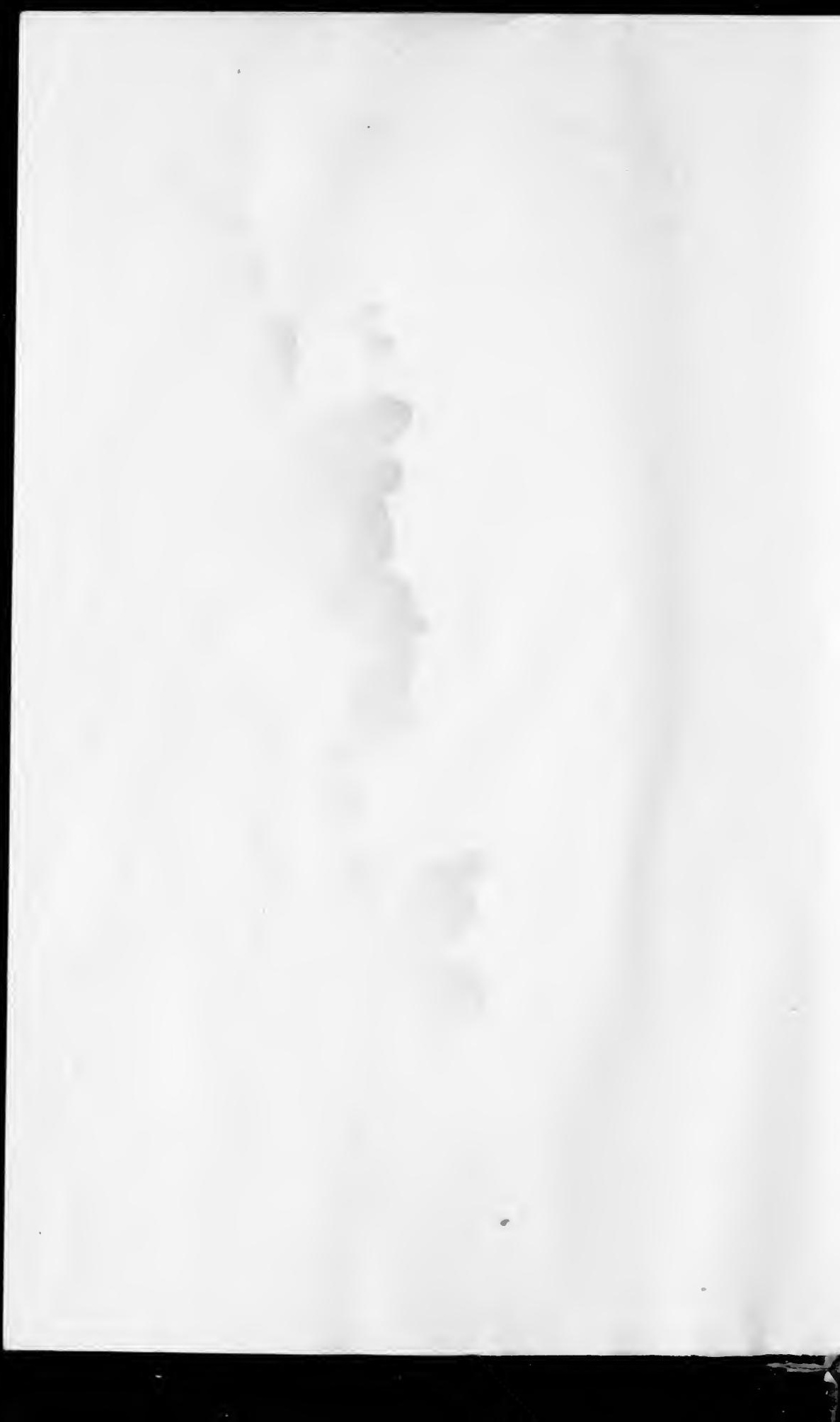




\section{BEGINNER'S GRAMMAR}

\section{OF THE \\ GREEK NEW TESTAMENT}

BY

WILLIAM HERSEY DAVIS, M.A., TH.D. ASSOCIATE PROFESSOR OF NEW TESTAMENT INTERPRETATION IN THE SOUTHERN BAPTIST THEOLOGICAL SEMINARY LOUISVILLE, KENTUCKY

HARPER \& BROTHERS PUBLISHERS NEW YORK AND LONDON 
COPYRIGHT, I923,

BY THE SUNDAY SCHOOL BOARD

OF THE SOUTHERN BAPTIST CONVENTION

BEGINNER'S GRAMMAR OF THE GREEK NEW TESTAMENT PRINTED IN THE UNITED STATES OF AMERICA

$$
\text { A-V }
$$


To

A. T. ROBERTSON, D.D., LL.D.,

INCOMPARABLE MASTER AND TEACHER OF THE GREEK NEW TESTAMENT

This Volume

is affectionately Dedicated 


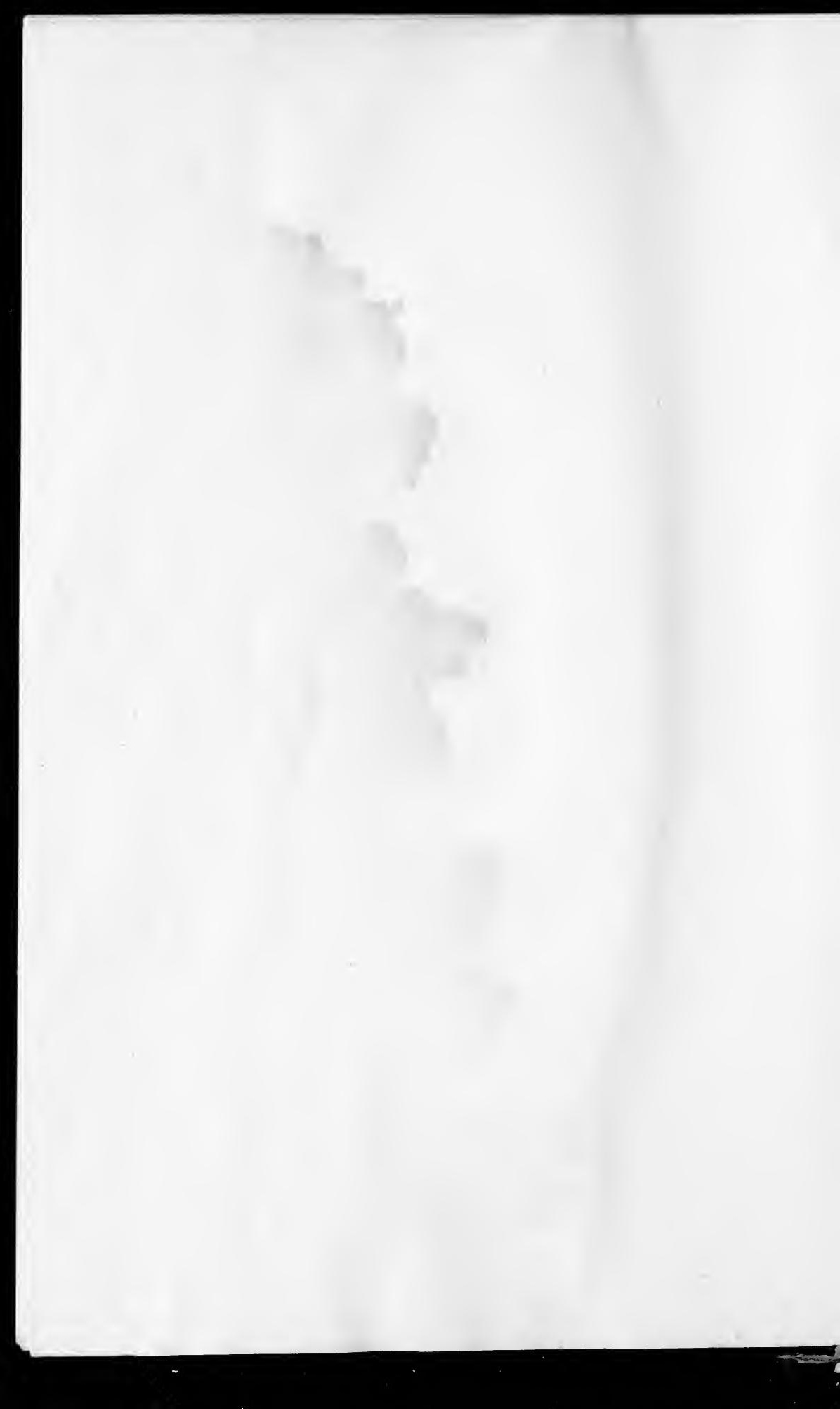




\section{INTRODUCTION}

It gives me the greatest pleasure to write some words of an introductory nature to the Beginner's Grammar of the Greek New Testament by my beloved colleague, Dr. W. H. Davis. The need of this book is urgent. Hardly a week goes by that I am not asked to recommend such a book to young ministers, to pastors, to laymen, to women, many of whom wish to learn how to read the Greek New Testament without the advantage of a teacher. There are a number of grammars that undertake to do this thing, but they all start in the wrong way, except Moulton's Introduction, which is not well suited to American schools.

It is a curious thing how traditionalism in linguistic teaching has held in slavery so many men who teach Greek today precisely as it was done a hundred years ago. The revolutionary progress made by Brugmann and Delbrück in comparative philology is left to one side for technical scholars. Professor Davis starts the student right. The standpoint of Thumb's revision of Brugmann's Griechische Grammatik is presented with clearness and precision. The student who starts with Davis's Beginner's Grammar can go right on to my Short Grammar of the Greek New Testament without a break or jolt. Then he will be ready for my Grammar of the Greek Nerw vii 
viii

Testament in the Light of Historical Research. It is only a step further to the Brugmann-Thumb Griechische Grammatik and in the same direction. In my experience of thirty-five years as a teacher of the Greek New Testament I have always had numbers of men who floundered over the cases, the prepositions, the tenses, the voices, the modes, because they had learned these basal things in the old unscientific way. It is like pulling eye-teeth for such a one to learn that the genitive is not the whencecase, but only the case of kind or genus, and that the ablative is the whence-case. If one gets it into his head that the root idea of tense is time, he may never get it out and he will therefore never understand the beauty of the Greek tense, the most wonderful development in the history of language. Professor Davis is absolutely at home in the new science of language and, I may add, is the most brilliant student of Greek that I have ever had. One should, if possible, take the college course in ancient Greek. He needs this background and this contact with the glorious period of the Greek language. But the New Testament is the chief glory of the Greek tongue, and one can begin it in the right way under Professor Davis's tutelage.

Professor Davis is a master of the papyri and so of the Koine in which the New Testament books are written. He is not giving the grammar of the literary Attic, but the grammar of the Koiné of the first century A.D. This fact is the second linguistic discovery that has revolutionized the study of the Greek New Testament. Comparative philology and 
the papyri discoveries have put the old grammars out of date and all the new ones that ignore the tremendous progress thus made. It is now known that the Greek of the New Testament is not literary Attic nor is it a peculiar Hebrew jargon or sacred Greek dialect. At bottom it is simply straight Koiné of the first century A.D. like that found in the inscriptions of Asia Minor and in the papyri of Egypt. The papyri give us many thousands of examples of the language of the life of the first century A.D. in Egypt. There are business contracts, bills, deeds, marriage contracts, wills, decrees, love letters, business correspondence, anything and everything that made up the life of the people of the time. These relics preserve the language of people of all degrees of culture. The Koiné means the language common to people everywhere, not merely the language of the common people. It was the means of communication all over the Roman Empire. The most of the papyri examples give the vernacular form of the Koiné, but there are specimens of the literary Koiné also. The New Testament is mainly in the vernacular Koiné, but it is the vernacular of men of great ability and some of them have a decided literary flavor, as we see in the writings of Luke, the Epistles of Paul, the Epistle to the Hebrews.

Language changes with the years if it is alive. Changes occur in the meaning of words, and here the papyri give very great help in showing what the words of the New Testament meant in everyday life. Dr. Davis himself has found over two thousand words in the papyri not given in any of the 
Greek lexicons. But the forms of the Koine show numerous changes from those in the Attic. Dr. Davis's Grammar gives the forms of the Koiné, not of the Attic Greek. Syntax shows some changes also, and these are given rightly.

There are Hebraisms and Aramaisms in the Greek New Testament, but the number is nothing like so great as was once thought to be the case. It is natural that Jews who spoke and wrote the Koiné should reveal here and there familiarity with $\mathrm{He}$ brew and Aramaic. Even Luke, probably a Greek, has the ear-marks of Aramaic sources and of knowledge of the Septuagint. But, in the main, the New Testament is written in the current Koiné, as one would expect.

It should be added that Dr. Davis confines himself to a Beginner's Grammar. He does not try to teach the ancient Attic on the one hand nor to go over the ground of my Short Grammar on the other. He definitely undertakes to prepare students for the Short.Grammar, and he does it with consummate skill. He supplies in masterly fashion the book that was needed. He will smooth the path for the beginner in the Greek New Testarient. He will make it so easy that one will wonder why he was so long starting on the road that leads one into the heart of the greatest of all the books of earth, the Greek New Testament.

Louisville, $K y$.

A. T. ROBERTSON 


\section{NOTE TO FIFTH EDITION}

In the present edition various corrections and a few additions have been incorporated. A revised and enlarged edition is planned to appear when circumstances warrant it. I thank my colleague, Dr. E. A. McDowell, Jr., for corrections and valuable suggestions. $\mathrm{He}$ is at work on a Koine reader which may be used in conjunction with the Grammar.

W. Hersey Davis

Louisville, Kentucky,

1942 


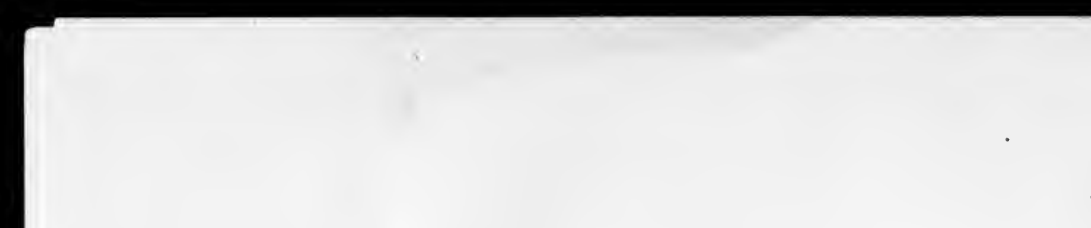




\section{PREFACE}

Dr. A. T. Robertson wrote in the Preface to his Short Grammar of the Greek New Testament: "Three types of New Testament grammars are needed: a beginner's grammar for men who have had no Greek training, an advanced and complete grammar for scholars and more critical seminary work, an intermediate handy working grammar for men familiar with the elements of Greek both in school and in the pastorate." This book is designed to meet the need for the first type. It is intended for those who are beginning the study of the Greek New Testament or have an imperfect knowledge of the essentials of the Greek of the New Testament, and to serve as a preparation for A Short Grammar of the Grizek New Testament (A. T. Robertson).

The book is a beginner's book. It is the result of class-room experience of many years. The need and preparation of a beginner's class in Greek has determined the method and order of presentation. The Greek of the New Testament is the Koine of the first century A.D. It is presented as such in this book. The historical development of the Greek language has been kept in mind.

No forms or words are given which do not occur in the Greek New Testament. All illustrations and sentences for translation have been taken from the ni 
New Testament. Those words which are of the most frequent occurrence are presented first.

In this book especial stress has been laid upon the meaning of the cases, the prepositions, and the tenses, wherein most beginner's books have been faulty.

The author wishes to record his great indebtedness to his teacher, Professor A. T. Robertson, D.D., LL.D., of the Southern Baptist Theological Seminary, for instruction and for invaluable assistance. Without his encouragement the work would not have been begun nor would it have been brought to completion. Whatever of worth this book may have, it owes much (if not all) to his rare scholarship and experience of many years as a teacher of the Greek New Testament. In fact his monumental work $A$ Grammar of the Greek New Testament in the Light of Historical Research is the authority from which this book drew at all points. Of course he is not at all responsible for any faults or errors which this book may contain.

The names of many writers whose works were consulted, the author cannot here recount, except Brugmann-Thumb (Griechische Grammatik).

In conclusion the author wishes here to express his thanks to his colleague, Professor F. M. Powell, A.M., Th.D., for valuable criticisms of a great part of the book in manuscript, to his friend, Dr. A. R. Bond, for expert criticism and preparation of the Index, and to his father, Rev. Q. C. Davis, Albemarle, N.C., for his sympathy and guidance.

Louisville, $K y$.

W. Hersey Davis 


\section{CONTENTS}

INTRODUCTION BY REV. PROF A T ROBERTSON, D PAgE PREFACE . . . . . . . . . . . . xi

\section{PART I: LESSONS}

LESSON

I The Alphabet . . . . . . . . Ig

II Vowels, Diphthongs, Breathing . 2 I

III ACCENT . . . . . . . . . . 22

IV Present Indicative Active . . . 25

V Second or O-Declension . . . . 27

VI Declension of o-Stems (Continued) . 30

VII Declension of o-Stems (Continued) . 32

VIII Declension of o-Stems (Concluded) • 35

IX Present Indicative Middle . . . 36

X Present Indicative Passive . • 39

XI Imperfect Indicative Active . . . 4I

XII Prepositions . . . . . . . . . 43

XIII Imperfect Indicative Middle and

$$
\text { PASSIVE . . . . . . . . . . . } 46
$$

XIV First Declension or Declension of $\alpha$-STEMS . . . . . . . . . . 49

XV First Declension (Continued) • . $5 \mathrm{I}$

XVI First Declension (Continued). . 53

XVII Adjectives of the First and Second

Declensions . . . . . . . . 56

XVIII Adjectives of the First and Second

Declensions (Continued) . . . . 59 xïi 
xiv

CONTENTS

LESSON

XIX Personal Pronouns and eiju, I am .

XX Third Personal Pronoun. ImperFECT OF siui . . . . . . . . 65

XXI Defective ("Deponent") Verbs. Demonstrative Pronouns oũtos AND ÉxEIVOS . . . . . . . . . 69

XXII Present Subjunctive Active . . . 73

XXiII Present Subjunctive Middle and Passive . . . . . . . . . . 75

XXIV Second Aorist Indicative Active ANd Middle . . . . . . . . 77

XXV Second Aorist Subjunctive Active AND MIDDLE

$8 \mathrm{I}$

XXVI Third Declension: Neuter SubSTANTIVES IN $-\mu \alpha \tau$. . . . . . 83

XXVII Future Indicative Active and MidDLE .

XXVIII Future Indicative Active and MidDLE (Continued)

XXIX Third Declension: Lingual Mute Stems

XXX Participles: The Present, Active, Middle and Passive . . . .

XXXI Participles: The Second Aorist Active and Middle . . . . . iol

XXXII Third Declension (Continued): Mutes and Liquids

106

XXXIII Third Declension (Continued): Liquid Stems in -ep (Syncopated). The Relative Pronoun . . . . Iio

XXXIV Third Declension (Continued): Stems in - . The Interrogative and Indefinite Pronouns . . II3

XXXV Third Declension (Continued):

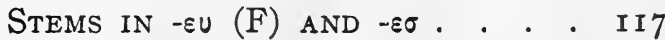




\section{CONTENTS}

XXXVI First Aorist Indicative Active and

Middle . . . . . . . . . .

XXXVII First Aorist Subjunctive Active and Middle . . . . . . . .

XXXVIII First Aorist Active and Middle Participle. Adjectives of the Third Declension. Declension of $\pi \tilde{\alpha} \varsigma$.

XXXIX Adjectives of the Third DeclenSION: STEMS IN -es. IRREgULAR

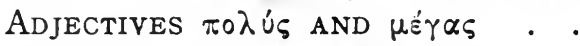

XL First Aorist Passive Indicative and Subjunctive. Future Passive InDICATIVE

XLI Aorist Passive (Continued) . . . 142

Xlit Some Irregular Substantives of the Third Declension. Some Uses of the Infinitive . . . . $\mathrm{I}_{4} 6$

XliI The Perfect and Pluperfect Indicative Active

XliV The Perfect Tense (Continued) . I54

XlV Reflexive Pronouns. Reciprocal Pronoun. Indefinite Relative Pronoun . . . . . . . . . $\mathrm{I}_{5} 8$

XlVI Future Indicative and Aorist Active AND Middle of Liquid VerbS I62

XLVII The Imperative Mode . . . . I66

XlViII Numerals. oujoil . . . . . . . I7I

XLIX Present System of Contract Verbs

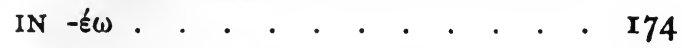

L Comparison of Adjectives and AdVERBS . . . . . . . . . . . 178

ZI Comparison of Adjectives and Adverbs (Continued). DECLENSION OP Adjbctives with Stems in -ov . . 182 
LESSON

LiI Present System of Contract Verbs

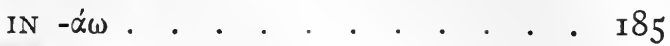

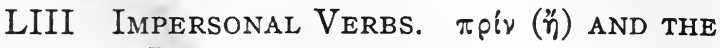

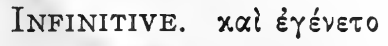

LiV Present System of Contract Verbs IN -ów

LV Conjugation of $\mu l$-Verbs: $\delta \delta \delta \omega \mu \ell$

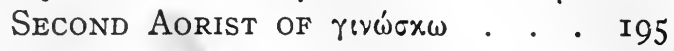

LVI Conjugation of $\mu_{l}$-Verbs (Continued): " $\sigma \tau \eta \mu$. Present Imperative of eifil. Second Aorist of

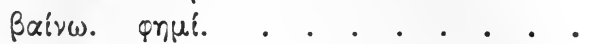

LVII Conjugation of $\mu l$-Verbs (Con-

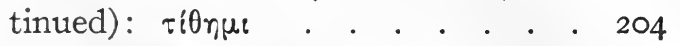

LVIII Conjugation of $\mu \iota$-Verbs (Continued): deinul, ouvinul. OTHER Verbs: Old Forms . . . . . 209

LiX The Optative Mode. Wishes . . 212

PART II: SUPPLEMENT TO PART I

A. Sounds and Writing . . . . . . . 217

B. Paradigms of Nouns . . . . . . . 2I9

C. Pronouns . . . . . . . . . . . . 224

D. Paradigms of the Verb . . . . . . 225

E. Classes of Verbs . . . . . . . 232

Note on Perfect Middle (Passive) of Mute Stems . • . • . • • • • • • • • 239 ENGLISH INDEX . • • • • • . 243 VOCABULARIES . . . . . . . 255 
PART I: LESSONS 


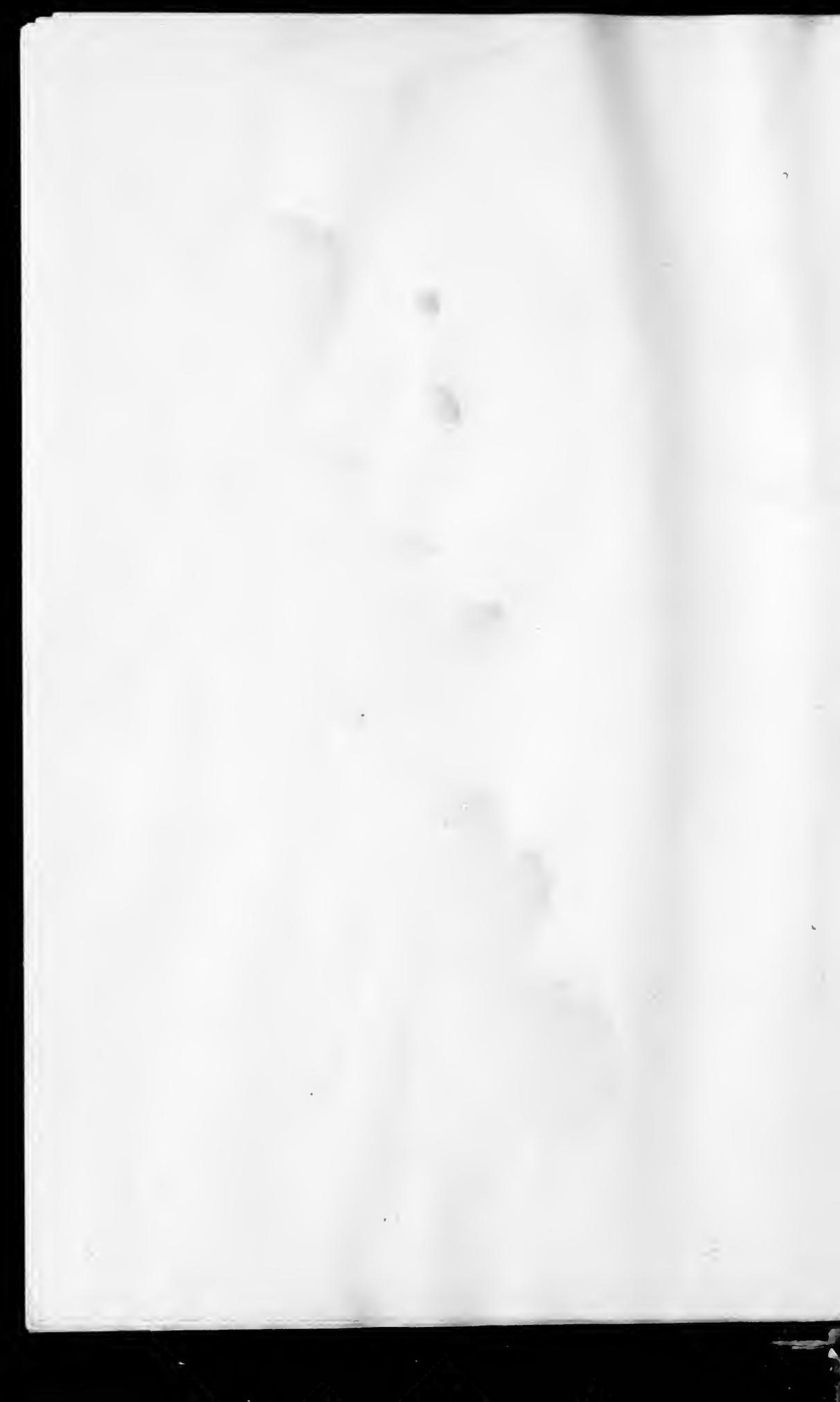




\section{BEGINNER'S GRAMMAR OF THE GREEK NEW TESTAMENT}

\section{LESSON I}

\section{The Alphabet}

1. The Greek alphabet had in the Koine or Hellenistic period twenty-four letters.

$\begin{array}{clcl}\begin{array}{c}\text { Form of capital } \\ \text { letters }\end{array} & \text { Name } & \begin{array}{c}\text { Form of small } \\ \text { letters }\end{array} & \text { Sounded as } \\ \text { A } & \text { alpha } & \alpha & \text { a in father } \\ \text { B } & \text { beta } & \beta & \mathrm{b} \text { in boy } \\ \Gamma & \text { gamma } & \gamma & \mathrm{g} \text { in go } \\ \Delta & \text { delta } & \delta & \mathrm{d} \text { in day } \\ \text { E } & \text { epsilon } & \varepsilon & \text { e (short) in met } \\ \text { Z } & \text { zeta } & \zeta & \mathrm{z} \text { in daze } \\ \text { H } & \text { eta } & \eta & \left\{\begin{array}{l}\mathrm{e} \text { in fête } \\ \mathrm{a} \text { in mate }\end{array}\right. \\ \Theta & \text { theta } & \theta & \text { th in thin } \\ \text { I } & \text { iota } & \checkmark & \left\{\begin{array}{l}\mathrm{i} \text { in police } \\ \mathrm{i} \text { in fit }\end{array}\right.\end{array}$

1 The sounds adopted as equivalent to the vowels in Greek are given for the sake of a consistent method of pronunciation. Already in the first century A.D. some of the vowels and diphthongs were sometimes pronounced alike, as in Modern Greek, and consequently were confused: thus $\varepsilon t, \imath, \eta, n, u$, ut, ot (being pronounced alike) were sometimes written one for another; so with $\varepsilon$ and $\alpha_{l}$, and $\circ$ and $\omega$. The confusion of vowels and diphthongs of $\bar{e}$ sound is called itacism.

There is clear evidence that in the first century A.D., $\beta$ had the twofold pronunciation of $b$ and $v$ (labiodental), as in Modern Greek, and $\gamma$ had begun to have the value of $y$ (the $j$ value of $i$ before $e$ and $i$ suunds). 
BEGINNER'S GREEK GRAMMAR

\begin{tabular}{|c|c|c|c|}
\hline $\begin{array}{c}\text { Form of capital } \\
\text { letters }\end{array}$ & Name & $\begin{array}{c}\text { Form of small } \\
\text { letters }\end{array}$ & Sounded as \\
\hline $\mathrm{K}$ & kappa & $x$ & $\mathrm{k}$ in $k$ eep \\
\hline$\Lambda$ & lambda & $\lambda$ & 1 in led \\
\hline M & $\mathrm{mu}$ & $\mu$ & $\mathrm{m}$ in $\operatorname{man}$ \\
\hline $\mathrm{N}$ & $\mathrm{nu}$ & $\nu$ & $\mathrm{n}$ in $n$ et \\
\hline$\Xi$ & $\mathrm{xi}$ & $\xi$ & $\mathrm{x}$ in $\operatorname{la} x$ \\
\hline $\mathrm{O}$ & omicron & 0 & o (short) in omit \\
\hline$\Pi$ & pi & $\pi$ & $p$ in peg \\
\hline $\mathrm{P}$ & rho & p & $\mathrm{r}$ in run \\
\hline$\Sigma$ & sigma & $\sigma \varsigma$ & $s$ in sit \\
\hline $\mathrm{T}$ & tau & $\tau$ & $\mathrm{t}$ in $\operatorname{ten}$ \\
\hline$\Upsilon$ & upsilon & $u$ & $\begin{array}{c}\mathfrak{u} \text { in Fr. } t u, \text { Ger. } \\
\text { Tür }\end{array}$ \\
\hline$\Phi$ & phi & $\varphi$ & ph in graphic \\
\hline $\mathrm{X}$ & chi & $x$ & $\begin{array}{l}\text { ch in Ger. ich, } \\
\text { Scotch loch } \\
\text { chasm }\end{array}$ \\
\hline$\Psi$ & psi & $\psi$ & ps in tops \\
\hline$\Omega$ & omega & $\omega$ & $\bar{o}$ (long) in note \\
\hline
\end{tabular}

$a$. At the end of a word sigma is written $\varsigma$, elsewhere $\sigma$; as in osıurós.

At first learn the form of the small letters only. Write each letter many, many times, pronouncing its name each time, until the whole alphabet can from memory be uttered and written without hesitation.

2. Every Greek word has as many syllables as it has separate vowels or diphthongs.

Learn the sound of each letter.

Pronounce aloud the following words:

$\begin{array}{llll}\theta \varepsilon-\lambda \omega, & \text { the-lō } & \gamma \rho \alpha-\varphi \eta, & \text { gra-phē } \\ \nu 0-\mu \circ 5, & \text { no-mos } & \lambda \varepsilon-\gamma \omega, & \text { le-gō }\end{array}$




$\begin{array}{llll}\beta \lambda \varepsilon-\pi \omega, & \text { ble-pō } & \sigma \omega-\zeta \omega, & \text { sō-zō } \\ \varphi t-\lambda \circ \varsigma, & \text { phi-los } & x \circ \sigma-\mu \circ \varsigma, & \text { kos-mos } \\ \nu \cup \xi, & \text { nux } & \varepsilon-\chi \omega, & \text { e-chō } \\ \alpha-\delta \varepsilon \lambda-\varphi \circ \varsigma, & \text { a-del-phos } & \psi U-\chi \eta, & \text { psu-chē } \\ \alpha \nu-\theta \rho \omega-\pi \circ \varsigma, & \text { an-thrō-pos } & \theta \varepsilon-\lambda \eta-\mu \alpha & \text { the-lē-ma }\end{array}$

\section{LESSON II}

\section{Vowels, Diphthongs, Breathing}

3. There are seven vowels: $\alpha, \varepsilon, \eta, \imath, 0, u, \omega . \eta$ is the long form of $\varepsilon$, and $\omega$ is the long form of 0 ; Eand 0 are always shorf, and $\omega$ always long. This list, then, corresponds in a way to the English a, e, i, o, u. $\alpha$, , $u$ are sometimes long and sometimes short; the long and short forms are not distinguished by separate characters.

4. A diphthong is two vowel sounds fused into one. The diphthongs are:

$\alpha_{t}=$ ai in aisle

$\alpha \cup\left\{\begin{array}{l}=\mathrm{au} \text { in Ger. haus } \\ =\mathrm{ou} \text { in house }\end{array}\right.$

$-\varepsilon \iota=$ ei in height

$o:=0 i$ in oil $\circ v=o u$ in group

$\varepsilon v=e u$ in $\mathrm{feud}$

$\eta v=$ approximately the same sound as eu.

$v_{t}=$ we

Also there are $\alpha, n, \omega$; but the (iota written underneath a vowel is called 'iota-subscript') does not affect the sound of the vowel.

5. Many Greek words begin with a sound equivalent to the English $h$. This sound is indicated by writing a sign (') called the rough breathing over a vowel or 
diphthong at the beginning of a word (over the second vowel of a diphthong). Thus $\delta \delta 0 \varsigma=$ hodos; $\varepsilon \dot{u}$ p $\odot \times \omega=$ heurisko. If an initial vowel or diphthong is not pronounced with an $h$, the sign ('), called the smooth breathing, is written over it. Thus $\alpha \times 0 v \omega=$ akouo; oupavos=ouranos. Initial $u$ always has the rough breathing.

6. Write the following in English (Roman) letters in accordance with the equivalents given in I and 4 .

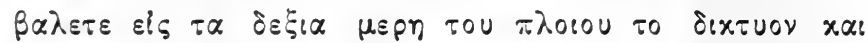

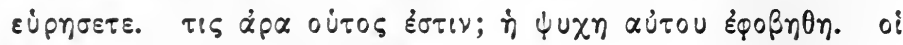
¿่े $\theta \rho \omega \pi 0 \iota$ है $\xi \eta \lambda \theta 0 \nu$ Éx rou oixou.

Write the following words in Greek characters: kai palin ērxato didaskein para tēn thalassan. kai sunagetai pros auton ochlos pleistos, hōste auton eis ploion embanta kathēsthai. periblepsamenos autous legei ide hẽ mētēr mou kai hoi adelphoi mou.

\section{LESSON III}

\section{Accent}

7. Most Greek words are written with accents. The accents are the acute ('), the grave ('), and the

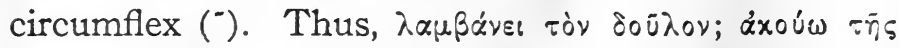
owvins. On inspection it will be noticed that the accent stands over the vowel of the accented syllable, and in a diphthong over the second vowel.

8. To us in English accent means a stress of the voice. Also to the native Greeks of today it means simply stress of voice. Originally, however, accents indicated the tone or pitch of the voice in pronouncing syllables. 
In pronunciation we make no distinction between the accents.

9. The last syllable of a word is called the ultima; the next to the last, the penult; and the one before the penult, the antepenult.

10. Learn the following verbs:

\begin{tabular}{|c|c|c|c|}
\hline 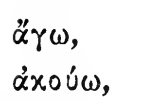 & $\begin{array}{l}\text { I lead, bring, go } \\
\text { I hear }\end{array}$ & $\begin{array}{l}0 \hat{\varepsilon} \lambda \omega, \\
\lambda \alpha \mu \beta \dot{\alpha} \nu \omega,\end{array}$ & $\begin{array}{l}\text { I wish, will } \\
\text { I take, receiv }\end{array}$ \\
\hline & I see, look at & 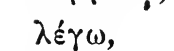 & $I s$ \\
\hline & I know & $\pi \dot{\varepsilon} \mu \pi \mathrm{c}$ & $I$ sen \\
\hline & I write & $\pi เ \sigma \tau \varepsilon \cup ́ \omega$ & I believe \\
\hline$\omega$ & I find & ${ }^{\prime} \chi \omega$, & I ha \\
\hline
\end{tabular}

Write and pronounce aloud each of these words (with the proper breathing and accent) fifteen to twenty times, associating with each word its meaning, as

ă $\gamma \omega$ á-gō, I lead. Notice how the breathing and accent are written together when they occur on the same syllable.

áxoúw, a-koú-o, I hear. Observe that the accent is written over the second vowel of the diphthong ou. See $7 \cdot{ }^{1}$

11. Observe that: I. Every initial vowel or diphthong has a breathing. 2. The acute accent stands on the penult. The accent of verbs is generally thrown as far back as possible from the last syllable. This is known as recessive accent. Here the position of the accent is determined by the last syllable:

1 Sections in the Lesson Part are referred to by the simple number (as 7). Sections in the Part dealing with Etymology are referred to by a section sign ( $\$$ ) before the number (as $\$ 6$ ). 
(I) If the last syllable is long, the accent falls on the penult. (2) If the last syllable is short, the accent falls on the antepenult. (3) A syllable is long if it contains a long vowel or diphthong; otherwise it is short. 3. The ending $-\omega$ in each of these words has the force of the personal pronoun $I$ in English.

12. I. In Greek the endings of verbs generally express the different persons, as $I$, thou (you), he, $w e$, ye (you), they. What is the ending of each verb in ro? The endings of verbs denoting person are called personal endings; they are fragments of old pronouns and are inseparable from the verb. But in English the personal pronouns are separate from the verb and are generally written before it.

In the case of most verbs the original personal endings in the singular of the present indicative are no longer apparent in the forms of the $-\omega$ verbs.

2. The verb affirms action (including "state"). A Greek verb has tense, mode, and voice. Tense expresses the state of the action of the verb; mode gives the manner of affirmation of the verb, how it is made; voice tells how the action of the verb is related to the subject. Verbs indicate affirmation by the personal endings.

The student should now begin to make a GreekEnglish and an English-Greek vocabulary arranged according to the alphabet. A good note book of convenient size should be used. 


\title{
LESSON IV
}

13.

\author{
Present Indicative Active
}

I. $\lambda \dot{\varepsilon} \gamma-\omega, I$ am saying, $\lambda \varepsilon^{\prime} \gamma-0-\mu \varepsilon \nu$, we are saying, I say

say

2. $\lambda \varepsilon^{\prime} \gamma-\varepsilon เ \varsigma$, you are say- $\lambda \varepsilon^{\prime} \gamma-\varepsilon-\tau \varepsilon$, ye are saying, ing, say say

3. $\lambda \dot{\varepsilon} \gamma-\varepsilon$, he, she, or it $\lambda \hat{\varepsilon}^{\prime} \gamma-0 \mathrm{vol}$, they are sayis saying, says ing, say

Infinitive, $\lambda \dot{\varepsilon} \gamma-\varepsilon เ \nu$, to be saying, to say

14. Only in the indicative mode in Greek do the tenses show time absolutely. The main idea of tense is the "kind of action," the state of action. Even in the indicative time is a secondary idea. Continued action, or a state of incompletion, is denoted by the present tense,-this kind of action is called durative or linear. The action of the verb is shown in progress, as going on. Observe that the indicative mode in Greek has practically the same declarative force as it has in English. In 13 the subject is represented as acting.

15. To conjugate a verb is to give all the variations in its terminations in the proper order. As given in I3, $\lambda \dot{\varepsilon} \gamma \omega$ is said to be conjugated in the present in. dicative active and present infinitive active.

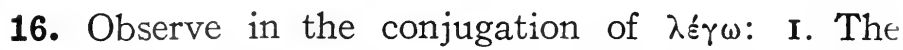
stem $\lambda \varepsilon \gamma$ - remains unchanged throughout. 2. A vowel follows this stem. 3. The vowel is o before endings that begin with $\mu$ or $\nu$, and $\varepsilon$ before other letters. This vowel (sometimes designated $\% / \epsilon$ ) is called the thematic vowel. 4. The thematic vowel 
is followed by an ending (the personal ending, see 12) clearly seen in $-\mu \varepsilon \nu$ and $-\tau \varepsilon$ of the first and second persons plural.

Thus, $\lambda \varepsilon \gamma-\varepsilon-\tau \varepsilon$ is composed of the stem $\lambda \varepsilon \gamma-$, the thematic vowel $-\varepsilon-$, and the personal ending $-\tau \varepsilon$.

17. The personal endings of the active voice, primary tenses, ${ }^{1}$ in their primitive form were these:

Singular

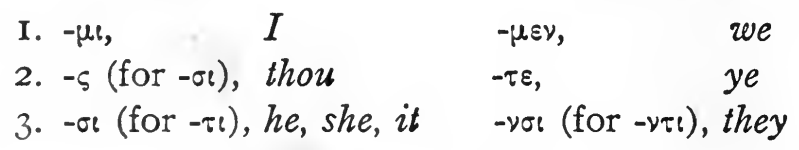

Plural
The personal endings are remnants of personal pronouns.

18. The thematic vowel with the personal ending may be exhibited thus:
I. $0-\mu s$
2. $\varepsilon-\varsigma$ (for $\varepsilon-\sigma \ell$ )
$0-\mu \varepsilon \nu$
3. $\varepsilon-\sigma !$ (for $\varepsilon \pi t)$
$\varepsilon-\tau \varepsilon$
$0-\nu \sigma t$ (for $0-\nu \tau t)$

a. The first person singular $-\omega$ is probably the result of dropping the personal ending $-\mu$ and the consequent lengthening of the thematic vowel o to $\omega$. b. $-\varepsilon \sigma \iota$ and $-\varepsilon \tau \iota$ of the second and third persons singular result in -sis and $-\varepsilon t$ respectively. $c$. In -ovo of the third person plural $\nu$ is expelled and 0 is lengthened to ou (such vowel change is called compensatory lengthening).

19. The resultant endings from combination of the thematic vowel and the personal ending are

1 The primary tenses are the present, the future, and the perfect; the secondary tenses are the imperfect, the aorist, and the pluperfect. 


\section{THE SECOND DECLENSION}

I. $-\omega, I$

2. $-\varepsilon เ \varsigma$, thou

3. - $\varepsilon 1$, he, she, it $-o \mu \varepsilon \nu, w e$

$-\varepsilon \tau \varepsilon, \quad y e$

-ouve, they

These forms must be mastered. Nothing short of absolute mastery of forms will answer the purpose.

20. The infinitive does not have personal endings.

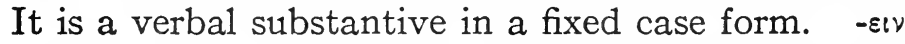
is the result of contraction of the thematic vowel $\varepsilon$ and the old locative ending $-\varepsilon \nu(-\varepsilon v t)$.

21. Exercises for pronunciation and translation.

I. Translate into English:

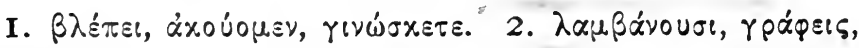

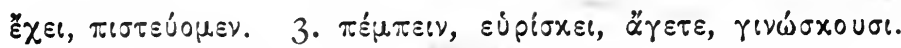

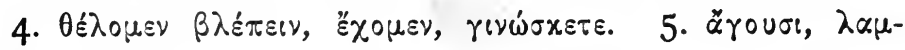

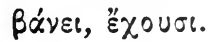

II. Translate into Greek:

I. We know, I see, he finds. 2. You send, they know, ye lead. 3. We wish to know, he hears. 4. They write, he has, you believe.

\section{LESSON V}

The Second Declension or Declension of o-Stems

22.

VOCABULARY

$$
\begin{aligned}
& \text { äpros, bread } \\
& \text { oporvos, throne }
\end{aligned}
$$

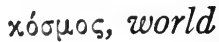

$$
\begin{aligned}
& \lambda(\theta \circ s, \text { stone } \\
& \text { ג6ros, word }
\end{aligned}
$$

vónos, law

$\therefore \not \chi \lambda \circ \varsigma$, crowd $\tau \delta \pi \circ \varsigma$, place xpbvos, time фi $\lambda \circ 5$, friend 
The student should take up the words of the vocabulary, one at a time, writing and pronouncing (aloud) each word with its proper accent and breathing until it can be spoken or written without hesitation. Learn thoroughly the meaning of each word. Do not take up a new word until the preceding word has been thoroughly mastered.

23. Observe: I. All the words in the vocabulary end in -os. 2. All these substantives belong to the odeclension. 3. They all have an acute accent on the penult.

24. In Greek all nouns (substantive and adjectives) are declined in one of three declensions. Substantives of the second declension have stems in -0-. The stem of a word is that part of it which remains virtually unchanged in all its forms.

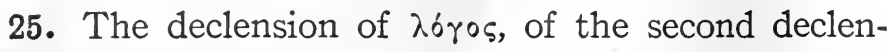
sion, is:

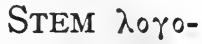

Singular

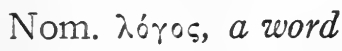

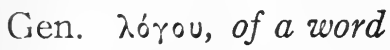

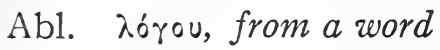

Loc. $\lambda \dot{6} \gamma \omega$, in, or at, a word

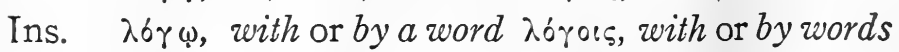

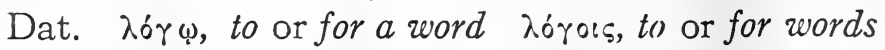

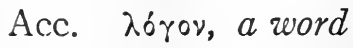

Voc. $\lambda o ́ \gamma \varepsilon, O$ word
Plural

$\lambda$ óro:, words

$\lambda 6 \gamma \omega \nu$, of words

$\lambda$ io $\omega \nu$, from words

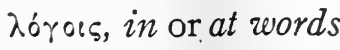

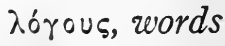

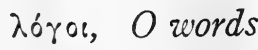

$a$. In the dat. sing. the ending $-\omega$ is for $-0+\alpha$ (dat. case-ending $)=\omega \iota=\omega$. In the loc. sing. the 


\section{THE SECOND DECLENSION}

ending $-\omega$ is for $-0+\imath$ (loc. case-ending) $=0 \imath=\omega t=\omega$. In the ins. sing. the ending $-\omega$ is for $-0+\alpha$ (ins. caseending $)=\omega$. Because the forms of these cases were pronounced alike, they early came to be written alike. $b$. The genitive and ablative cases early came to have their forms alike. c. The loc., ins., and dat. plural have the ending of the instrumental case -oıs. $d$. So far as the form goes the

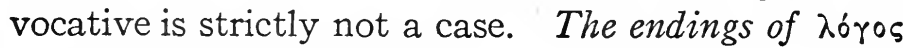
must be absolutely mastered.

26. I. Note that the accent on doros remains on the same syllable throughout the declension. In the declension of a substantive the accent is kept, if possible, on the same syllable on which it rests in the nominative case.

2. The accent of the nominative case must be learned by observation of each word.

27. It is to be observed, from 25, that in Greek there are eight cases (appearing under five caseforms): Nominative, Genitive, Ablative, Locative, Instrumental, Dative, Accusative, and Vocative. The nominative is the case of the subject, corresponding roughly to the English nominative. The genitive is the specifying case, expressed in English by the possessive or the objective with of. The ablative is the whence case (origin or separation), expressed in English by off, out, from, away, etc. The locative is the in case, corresponding to the English in, on, among, at, by. The instrumental is the case of means or association, expressed in English by with, by, etc. The dative is the case of personal 
interest (denoting advantage or disadvantage), corresponding to the English to or for, or indirect object. The accusative is the case of extension (whether of thought or verbal action), corresponding roughly to the English direct object. The vocative is the case of address.

28. In Greek the case-endings of nouns express the relation of words to each other, and to other parts of the sentence. In English this relation is generally expressed by prepositions (such as of, for, at, on, in, $b y$, etc.) and position of words.

29.

EXERCISES

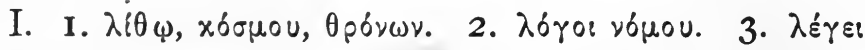

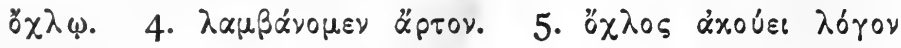
vópou.

II. I. In a place, of a world. 2. For a friend, laws of thrones. 3. He takes a stone. 4. We have bread for a world. 5. Ye speak words to crowds.

\section{LESSON VI}

\section{Declension of o-Stems (Continued)}

30. VOCABULARY

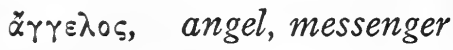

$\ddot{\alpha} \nu \theta \rho \omega \pi \circ$ s, man

$\dot{\alpha} \pi \sigma \sigma \tau 0 \lambda .5$, apostle

$\delta\llcorner\delta \dot{\alpha} \sigma x \alpha \lambda \circ \varsigma$, teacher

$\theta \dot{\alpha} \nu \alpha \tau \circ s, \quad$ death

xúpros, Lord

In $\ddot{\alpha} \gamma \gamma \varepsilon \lambda \circ \varsigma$ the first $\gamma$ is pronounced like $n g$. $\gamma$ is always pronounced $n g$ when it comes before $x, \gamma$, $\chi$, or $\xi$. Some words of the vocabularies so far given occur Iooo times in the New Testament. 
31. Nouns of the o-declension whose nominatives end in -os are generally masculine in gender (rarely feminine).

32. Declension of the masculine article $\delta$ the, and $\ddot{\alpha} \nu 0 \omega \pi \circ \varsigma$ :

\section{STEM $\alpha \dot{\alpha} \theta \rho \omega \pi 0-$}

\section{Singular}

Plural

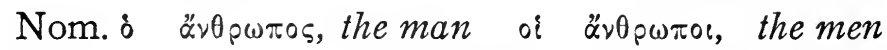
Gen. $\tau \circ \tilde{u} \dot{\alpha} \nu \theta \rho \dot{\omega} \pi \circ u$, of the man $\tau \tilde{\omega} \nu \dot{\alpha} \nu \rho \rho \dot{\omega} \pi \omega \nu$, of themen

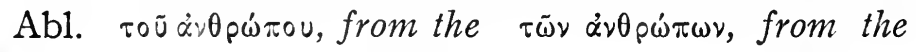
man

men

Loc. $\tau \tilde{\varphi} \dot{\alpha} \nu \theta p \dot{s} \pi \omega$, in or at $\tau$ oís $\dot{\alpha} \nu \theta p \dot{\omega} \pi 015$, in or at the man

the men

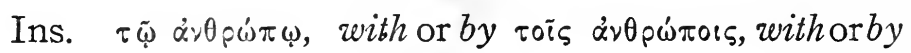
the man

the men

Dat. $\tau \tilde{\varphi} \dot{\alpha} \nu \theta p \dot{\omega} \pi \omega$, to or for $\tau 0 \tilde{\iota} \varsigma \dot{\alpha} \nu \theta \rho \dot{\omega} \pi 01 \varsigma$, to or for the man the men

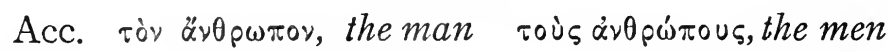
Voc. $\quad \ddot{2} \theta \rho \omega \pi \varepsilon, O$ man $\quad \ddot{\nu}, \theta \rho \omega \pi \circ, O$ men

33. Observe: In the declension of $\ddot{\alpha} v \theta \rho \omega \pi \circ \varsigma$ : I. The acute accent stands on the antepenult in the nominative case, and the ending -05 is short. 2. When the ultima becomes long, as in the endings $-o v,-\omega,-\omega \nu,-015,-0 \cup 5(3,4$, and II, (3)) the accent moves to the penult. Cf. 23, 2. 3. Final -ot, although a diphthong, is considered short in determining the place of accent in the 0 - declension.

34. The acute accent may stand on the ultima, penult, or antepenult. 


\section{BEGINNER'S GREEK GRAMMAR}

I. The acute accent cannot stand on the antepenult when the ultima is long, but may stand on the penult.

2. The acute accent (') on a final syllable is changed to the grave (") when another word immediately follows without any intervening mark of punctuation.

35. Note: I. The article in the nominative case has no accent, - it is to be pronounced with the following word. 2. The circumflex accent is written over the gen., abl., loc., inst., dat. cases of the article; and the syllable on which it stands is long. 3. The grave accent in the acc. case of the article. 4. The rough breathing over the nom. case of the article.

36. The definite article $\delta$, the, is an adjective, and, like all adjectives in Greek, it is declined and agrees in gender, number, and case with the word it modifies.

37.

EXERCISES

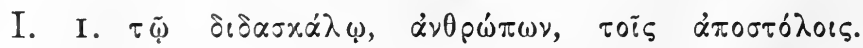

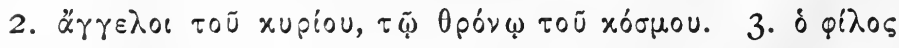

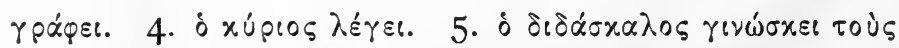

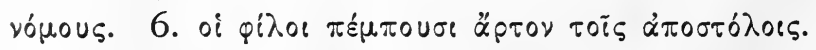

II. I. With a stone, at the place, of the world. 2. He sees the crowd. 3. The man wishes to find bread for the apostles. 4. We see the friends of the Lord. 


\section{LESSON VII}

Declension of o-Stems (Continued)

38.

VOCABULARY

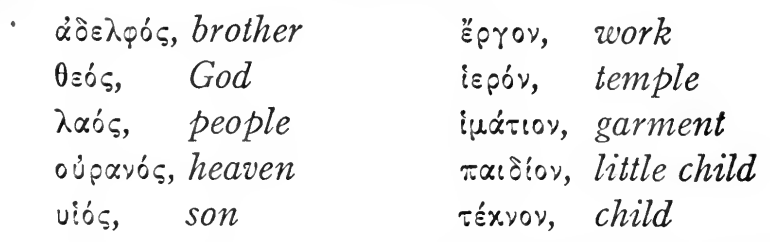

The diphthong in vi with the rough breathing, as in viós, is pronounced like hwee.

39. Declension of oujpavós.

\begin{tabular}{|c|c|}
\hline \multicolumn{2}{|c|}{ STEM oupavo- } \\
\hline Singular & Plural \\
\hline Nom.ouparós & oupavoí \\
\hline Gen. oúpavoũ & 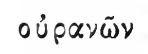 \\
\hline Ab1. oủpavoũ & 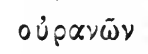 \\
\hline Loc. oúpav̄̄ & oúpavo:s \\
\hline Ins. oúpav ب़ & oupavois \\
\hline Dat. oujpavị & oúpavois \\
\hline Acc. oúpavóv & oúpavoús \\
\hline Voc. oủpavé & oủparoí \\
\hline
\end{tabular}

40. Note that: I. In every gen., ab1., loc., ins., and dat. the acute (') is changed to the circumflex ("). 2. In the diphthongs -ov and -ois the circumflex is written over the second vowel. See 7 .

41. A long ultima in the gen., abl., loc., ins., and dat. cases, if accented, receives the circumflex accent. 42. Learn the declension of the neuter substantive Éprov with the neuter article to the. Neuter substantives of the 0 - declension have their nominatives sing. in $-\%$ 


\begin{tabular}{|c|c|}
\hline Singular & Plural \\
\hline Nom. $\tau \dot{~ E ̈ p r o v ~}$ & $\tau \dot{\alpha}$ हैp $\gamma \alpha$ \\
\hline 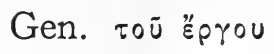 & $\tau \tilde{\omega} \nu \stackrel{\varepsilon}{\varepsilon} \rho \gamma \omega \nu$ \\
\hline Abl. тоü Ëprou & 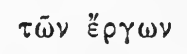 \\
\hline 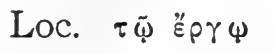 & tois हैprols \\
\hline Ins. $\tau \tilde{\varphi}$ हैp $\varphi$ & tois हैprols \\
\hline 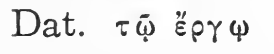 & tois हैprols \\
\hline 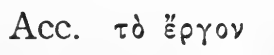 & $\tau \grave{\alpha}$ हैp $p \alpha$ \\
\hline Voc. Ëprov & Ëp $\alpha$ \\
\hline
\end{tabular}

43. It is to be observed in the declension of Ěprov: I. The nom., acc., and voc. cases in the singular have the same ending, -ov; and the same cases in the plural have the ending $-\alpha .2$. The inflection of the other cases is the same as that of masculine substantives.

44. The neuter article, $\tau$ to the, differs in its inflection from the masculine article only in the nom. and acc. cases.

45.

EXERCISES

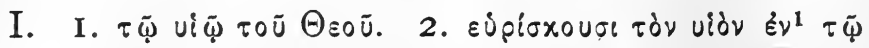

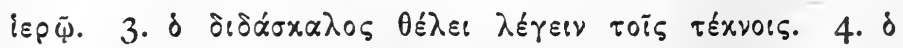

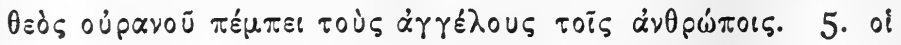

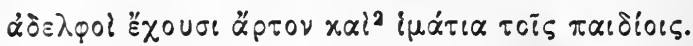

II. I. For the brothers and of the brothers. 2. The friend finds the garments of the children. 3. We see the son in the temple. 4. The teacher wishes to speak to the little child.

$\therefore c^{1} \varepsilon^{2}$, in, is used with the locative case.

${ }^{2}$ xal, and, used more times than any other conjunction in the New Testament. 


\section{LESSON VIII}

\section{Declension of o-Stems (Concluded)}

46.

VOCABULARY

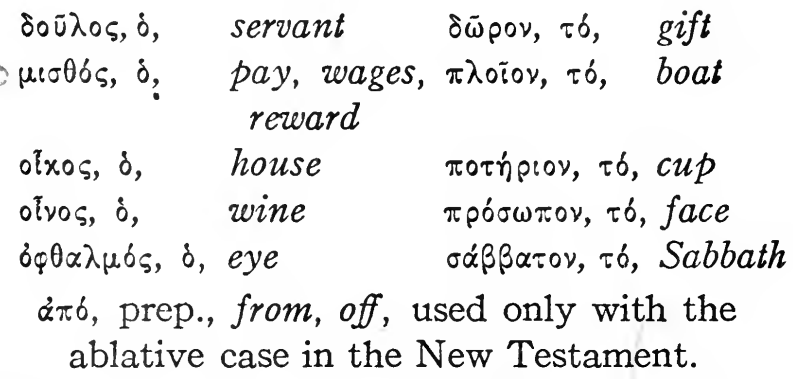

Notice that when the breathing and circumflex accent belong to the same vowel, the circumflex is written directly over the breathing, as in oixos, etc.

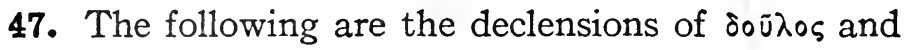

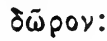

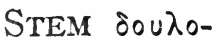

Singular

Nom. $\delta 0 \tilde{\lambda} 05$

Gen. soúrou

Abl. $\delta 0 \cup \operatorname{\lambda ou}$

Loc. $\delta 0 \hat{u} \lambda \varphi$

Ins. $\delta 0 u \dot{\lambda} \varphi$

Dat. $\delta 0 \hat{\nu} \lambda \varphi$

Acc. $\delta \circ \tilde{u} \lambda \circ \mathrm{V}$

Voc. $\delta \circ \tilde{u} \lambda \varepsilon$
Plural

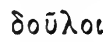

$\delta 0 u \dot{\lambda} \omega \nu$

$\delta 0 u \dot{\lambda \omega \nu}$

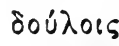

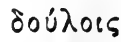

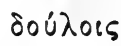

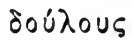

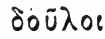

STEM $\delta \omega \rho o-$

Singular Plural

Nom. $\delta \tilde{\omega} \rho \circ \nu \quad \delta \bar{\omega} \rho \alpha$

Gen. $\delta \omega \dot{\omega} \rho 0 u \quad \delta \dot{\omega} \rho \omega \nu$

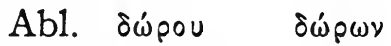

Loc. $\delta \omega \dot{\rho} \varphi \quad \delta \omega$ pors

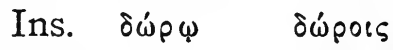

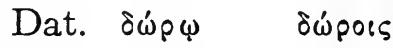

Acc. $\delta \bar{\omega} \rho \circ \nu \quad \delta \bar{\omega} \rho \alpha$

Voc. $\delta \tilde{\omega} \rho 0 \nu \quad \delta \bar{\omega} \rho \alpha$

48. Observe that: I. The circumflex accent $\left(^{-}\right)$occurs on a long syllable only. 2. When the circumflex accent is written on the penult, the last syllable 
is short. (3.) When the last syllable becomes long, the circumflex accent is changed to the acute. Cf. 26, I.

49. The circumflex accent may stand on the ultima or the penult. It cannot stand on the antepenult. The circumflex accent cannot stand on the penult when the ultima is long. When the ultima is short and the penult is long, the penult takes the circumflex accent, if it is to be accented.

50.

EXERCISES

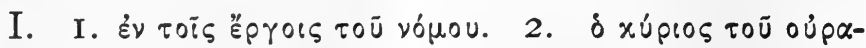

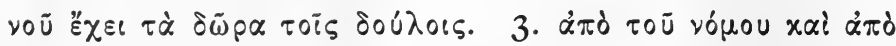

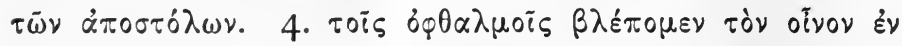

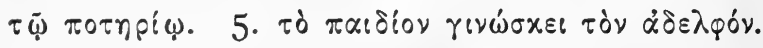

II. I. In the world and in the temple. 2. From the temple and from heaven. 3. The apostles receive bread for the servants. 4. We know the law and believe. 5. The son wishes to speak to the children.

\section{LESSON IX}

\section{Present Indicative Middle}

51. The Greek verb has three voices: active, middle, and passive. The active and passive voices are used as in English; the active voice represents the subject as acting; the passive voice represents the subject as acted upon.

52. The middle voice represents the subject as acting with reference to himself. Thus: I. As 
acting directly on himself (direct middle): $\lambda_{0}$ ú $\omega, I$

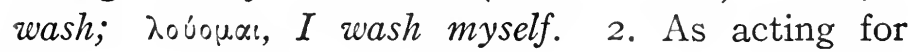
himself or for his own interest in some way: $\dot{\alpha} \gamma \circ \alpha^{\prime} \zeta_{\omega} \omega$, I buy; àyopásoua, I buy for myself.

a. Precisely how the subject acts with reference to himself, the middle voice per se does not tell. This precise relation is determined by the meaning of the verb itself and the context. $b$. Often it is impossible to translate the shade of meaning given by the middle. Yet in some verbs there is a bold change in meaning.

53. The conjugation of the present indicative middle of $\lambda 0^{\prime} \omega, I$ wash, is

\section{Singular Plural}

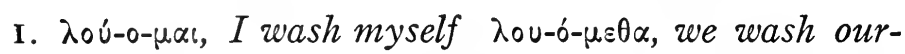
selves

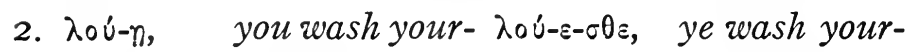
self selves

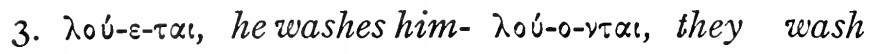
self themselves

Present infinitive middle $\lambda_{\circ} \dot{u}-\varepsilon-\sigma \theta \alpha$, to wash oneself.

Observe that the appended translation is the direct middle. If àyopáboual I buy for myself, etc., were given, the indirect middle would be seen.

54. The primary middle personal endings are:
Singular
Plural
I. $-\mu \alpha,, I$
$-\mu \varepsilon \theta \alpha$, we
2. $-\sigma \alpha \ell$, thou (you)
$-\sigma \theta \varepsilon, \quad y e$
3. $-\tau \alpha$, he, she, it
$-\nu \tau \alpha$, they 


\section{BEGINNER'S GREEK GRAMMAR}

The thematic vowel $(\% / \epsilon)$ and personal endings:

$$
\begin{aligned}
& \text { I. }-o \mu \alpha \ell \quad-o \mu \varepsilon \theta \alpha \\
& \text { 2. }-\varepsilon \sigma \alpha 6 \quad-\varepsilon \sigma \theta \varepsilon \\
& \text { 3. - } \tau \tau \alpha l \quad-o \nu \tau \alpha \iota
\end{aligned}
$$

Observe in the conjugation of $\lambda_{0} \cup \omega$ that:

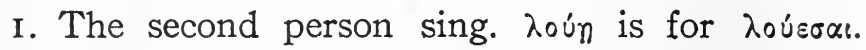
$-n$ arose from the dropping of $\sigma$ and the contraction of $\varepsilon$ and $\alpha_{\imath}=\eta \imath=n$. Rarely is $\varepsilon l$ found instead of n.

2. $\alpha_{l}$ in these personal endings is considered short, hence the accent on the antepenult. The same principle of accent is to be observed as in II and $\mathrm{I} 3$.

3. The thematic vowel $(\% / \epsilon)$ is found as in the active voice.

4. The present middle infinitive ending is $-\sigma \theta \alpha$. The $\alpha_{t}$ is considered short.

55.

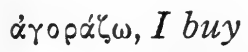

$\check{\alpha} \pi \tau \omega, \quad I$ fasten to

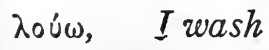

$\nu\{\pi \tau \omega, \quad I$ wash

$\pi \alpha u ́ \omega, \quad I$ stop

$\varphi \cup \lambda \alpha ́ \sigma \sigma \omega$

\section{VOCABULARY}

a'yopá̧oua, I buy for myself

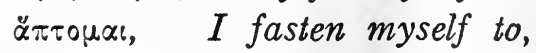
touch

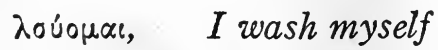

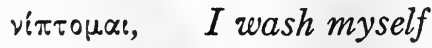
$\pi \alpha \dot{o} \mu_{\alpha}, \quad I$ stop myself, cease

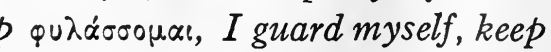
myself

This vocabulary is given as a simple illustration of the middle. Any verb may be used in the middle voice. 
56.

EXERCISES

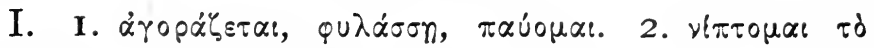

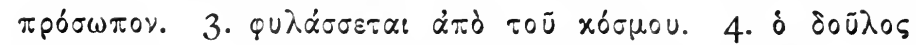

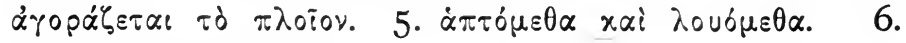

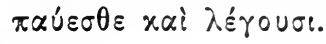

II. I. He ceases, they wash themselves. 2. You buy bread for yourself. 3. The apostle washes his (the) face. 4. The child guards himself from the man.

\section{LESSON $\mathrm{X}$}

\section{Present Indicative Passive}

57.

VOCABULARY

$\beta \alpha \dot{\lambda} \lambda \omega, \quad I$ throw

$\delta เ \delta \dot{\alpha} \sigma \times \omega$, I teach

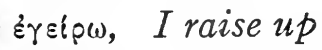

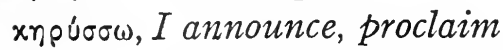

zis, prep., into, used with the accusative only. i $\pi 6$, prep., used with the ablative (see 86), by; with the accusative, under.

58. The passive voice is later than the active and middle and did not develop distinctive personal endings. The middle and passive are the same in form, except in the future and aorist. For the meaning of passive see $5 \mathrm{I}$.

59. The following is the present indicative passive of $\lambda$ ú I loose. 


\section{BEGINNER'S GREEK GRAMMAR}

Singular

I. $\lambda \dot{u}-0-\mu \alpha_{l}, \operatorname{I~am~(being)~} \lambda u-b-\mu \varepsilon \theta \alpha$, we are (being) loosed

2. $\lambda \dot{u}-n$ you are (being) loosed

3. $\lambda \dot{u}-\varepsilon-\tau \alpha$, he is (being) loosed

\section{Plural}

loosed

$\lambda \dot{u}-\varepsilon-\sigma \theta \varepsilon, \quad y e$ are (being) loosed

$\lambda \dot{u}-0-\nu \tau \alpha \iota$ they are (being) loosed

Present passive infinitive $\lambda \dot{u}-\varepsilon-\sigma \theta \alpha$, to be loosed.

60. It is to be observed that the present passive voice uses the present middle endings. The present middle and passive voices have the same form.

61. Generally the context will make clear whether the middle or passive voice is meant.

62.

\section{EXERCISES}

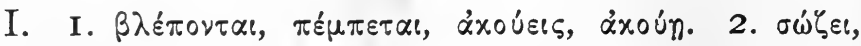

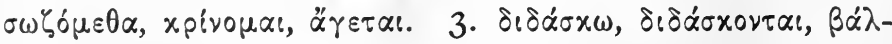

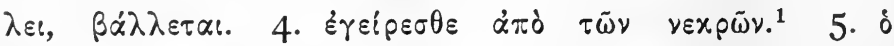

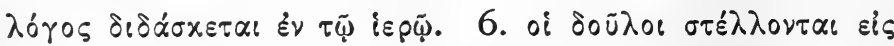

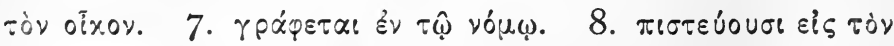

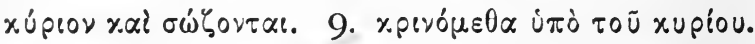

" II. I. He sends and is sent. 2. He believes and is saved. 3. The word is proclaimed in the temple. 4. The son of man is judged. 5. The stone is thrown into the house. 6. The bread is taken from the apostle. 7. You are judged by the son of man.

1 vexpbs, dead. 


\section{LESSON XI}

\section{Imperfect Indicative Active}

63.

VOCABULARY

alp $\omega, \quad$ I take up, bear $\quad \mu \varepsilon \dot{\varepsilon} \lambda \omega, I$ am about (or go$\beta \alpha \pi \tau i \zeta \omega, I$ baptize $\varepsilon \sigma \theta ! \omega, \quad I$ eat

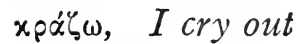
ing) to do something $\mu$ év $\omega$, I remain

64. The imperfect tense is made on the present

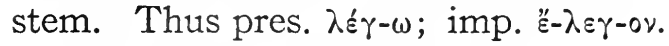

65. The imperfect indicative represents an action as going on in past time (durative or linear action in past time),- this action may be simultaneous, prolonged, descriptive, repeated, customary, interrupted, attempted, or begun, according to the context and the meaning of the verb itself.

66. The personal endings of the secondary ${ }^{1}$ active tenses are:
Singular
Plural
I. $->$
$-\mu \varepsilon \nu$
2. -5
$-\tau \varepsilon$
3. none
$-\nu$ or $-\sigma \alpha \nu$.

67. The imperfect indicative active of $\lambda_{0} \cup$ w, I wash:

Singular

Plural

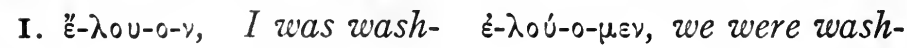
ing ing

2. $\varepsilon-\lambda \circ \cup-\varepsilon-\varsigma$, you were $\varepsilon-\lambda \circ u ́-\varepsilon-\tau \varepsilon$, ye were washwashing ing

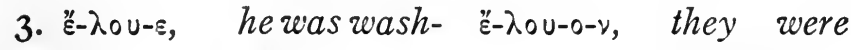
ing washing

${ }^{1}$ See 17 , footnote 1 . 
$a$. In the third pers. plu. the form $\varepsilon-\lambda \circ u^{\prime}-0-\sigma \alpha \nu$ is sometimes found. $b$. In the plural forms like

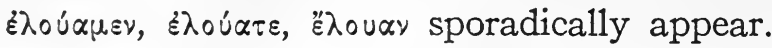

68. Observe that: I. The thematic vowel is $\%$ as in the present indicative. 2. The third person sing. has no personal ending. 3. Before the stem nouis $\varepsilon$. This $\varepsilon$ is called the augment.

69. The augment is probably an old adverb for "then." The augment in the imperfect places linear action in past time. At times it is difficult to translate this Greek tense into English, because of the absence of a true imperfect in English.

70. The indicative of the secondary (or historical) tenses, besides having different personal endings, has also an augment. This augment is of two forms: I. If the stem begins with a consonant, the vowel $\varepsilon$ is commonly prefixed-called syllabic augment. 2. But if the stem begins with a vowel, that vowel is lengthened-called temporal augment. Thus:

$\alpha$ becomes $\eta \quad(\alpha$ becomes $n)$

$\varepsilon$ becomes $\eta$ (al becomes $n$ ) [except in 2 Ti. i:I6, - becomes $\omega$ ( $\alpha u$ becomes $\eta u$ ) where it remains $\alpha$ ] $\checkmark$ becomes i

$\checkmark$ becomes $\bar{u}$

$\varepsilon \iota$ may become $n$, but remains $\varepsilon$ in New Testament. $\varepsilon u$ may become $\eta u$, but usually remains $\varepsilon u$ in New Testament.

o generally becomes $\psi$, but sometimes remains or in New Testament.

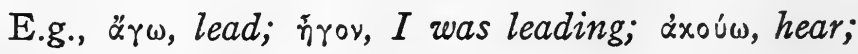




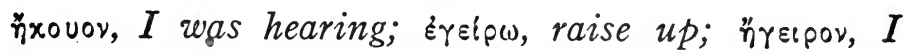

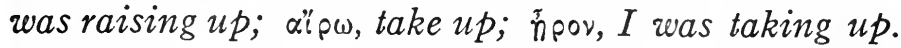

$\theta \dot{\varepsilon} \lambda \omega$ has $\eta$ in the imperfect, $\ddot{\eta} \theta \varepsilon \lambda$ ov. Sometimes $\mu \varepsilon \hat{\varepsilon} \lambda \lambda \omega$ has $\eta$, thus $\eta \mu \varepsilon \lambda \lambda$ ov.

71.

Exercises

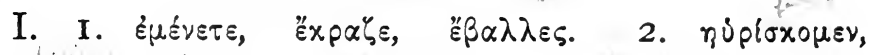

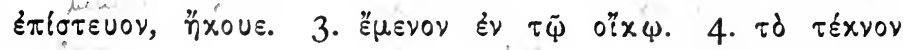

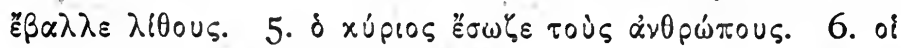

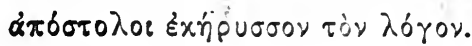

II. I. You were saying. 2.' They were eating the bread. 3. The teacher was sending garments for the children. 4. He was taking up the child from the boat. 5. The men were leading the servants into the house.

\section{LESSON XII}

\section{Prepositions}

72.

VOCABULARY

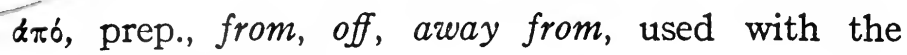
ablative only.

eis, prep., into, used with the accusative only.

$\varepsilon x(\xi \xi)$, prep., out, out of, from within, used with the ablative only. $\xi \xi$ before words beginning with a vowel.

iv, prep., in, used with the locative only. The resultant meaning of $\varepsilon v$ and the locative is sometimes instrumental.

$\pi \alpha \rho \alpha$, prep., beside, used with the locative, ablative, and accusative. 
oúv, prep., with, used with the instrumental only. $\dot{\alpha} \pi \circ \sigma \tau \dot{\varepsilon} \lambda \lambda \omega, \quad$ send forth

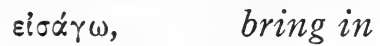

Ex $\beta \alpha \lambda \lambda \lambda \omega, \quad$ throw out, cast out

$\pi \alpha \rho \alpha \lambda \alpha \mu \beta \alpha \dot{\nu} \omega$, take, receive

ouvárw, gather together

Some prepositions do not have an accent. Words which do not have an accent are called proclitics and are to be pronounced with the following words.

73. Prepositions are adverbs specialized to define more clearly the meanings of cases, many of which come to be used in composition with verbs.

Prepositions were originally free adverbs. These adverbs were brought gradually into closer relation with nouns, and many of them into a closer connection with verbs.

74. Prepositions are used to bring out more clearly the idea of case. They help the cases; the case calls in the preposition to aid in expressing more sharply the meaning of the case. "It is the case which indicates the meaning of the preposition, and not the preposition which gives the meaning to the case." Then, strictly speaking, prepositions (in Greek) do not "govern" cases. Take $\pi \alpha \rho \alpha$, meaning beside, for example: with locative, $\pi \alpha \rho \dot{\alpha} \tau \tilde{\varphi} \delta \delta \dot{\lambda} \lambda \omega$, by or at the side of the servant; with the ablative, $\pi \alpha \rho \dot{\alpha} \tau \circ \tilde{u} \delta \circ u$ inou, from the side of the servant; with the accusative,

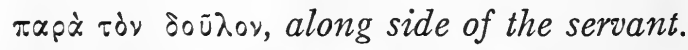

$\checkmark a$. The cases used with prepositions are the ablative, genitive, locative, instrumental, and accusative. 
$b$. The dative is not used with any of the preposi-

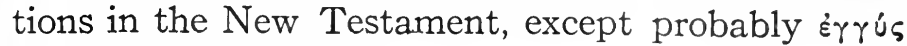
(Acts ix:38) and $\varepsilon$ eेt.

(75. In composition with verbs the preposition has commonly two uses.

I. The preposition is merely local: e.g., $\varepsilon x-\beta \dot{\alpha} \lambda \lambda \omega$, I throw out, cast out; $x \alpha \tau \alpha \beta \alpha i v \omega, I$ am going down.

2. The preposition intensifies or completes the idea of the verb: e.g., cs $s \theta^{\prime} \omega, I$ eat; $x \alpha \tau \varepsilon \sigma \theta i \omega$, I eat up (down). This is called the "perfective" force of the preposition.

$a$. Sometimes prepositions change the meaning of the verb and blend with it.

76. When a preposition ends in a vowel, as $\alpha \pi \dot{\alpha}, \pi \alpha \rho \alpha$, the final vowel is dropped before a verb that begins with a vowel: e.g., $\pi \alpha \rho \varepsilon$ é $\omega$ is for $\pi \alpha \rho \alpha+\varepsilon \chi \omega, I$ provide, supply. When compound verbs receive the augment, the final vowel of the preposition is dropped: e.g., $\pi \alpha \rho \alpha \lambda \alpha \mu \beta \alpha^{\prime} \nu \omega, I$ receive; $\pi \alpha \rho \varepsilon \lambda \alpha \dot{\alpha} \beta \alpha \nu \circ \nu$, $I$ was receiving; " $\dot{\alpha} \pi \circ \tau \varepsilon \dot{\varepsilon} \lambda \lambda \omega$, I send forth, $\dot{\alpha} \pi \varepsilon \sigma \tau \varepsilon \lambda \lambda \circ \nu$, $I$ was sending forth; $\kappa \alpha \tau \dot{\alpha} \gamma \omega, \kappa \alpha \tau \hat{\eta} \gamma \circ \nu$.

$a$. The prepositions $\pi \varepsilon p !$ and $\pi \rho b$ do not drop their final vowel; e.g., $\pi \rho \circ \alpha \dot{\gamma} \omega, I$ go before; $\pi \varepsilon \rho\llcorner\alpha \dot{\gamma} \omega$, I go about.

77.

\section{EXERCISES}

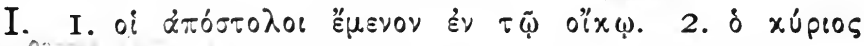

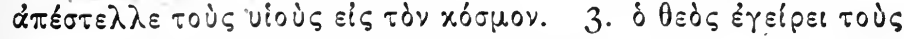

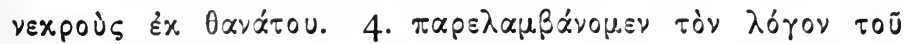

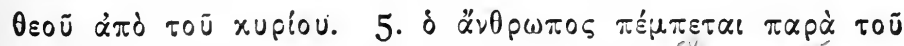

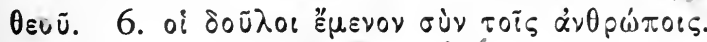


II. I. He was sending the child out of the boat. 2. The child was throwing stones into the house. 3. The servants were eating up the bread. 4. The Lord was sending forth the apostles. 5. The teacher is about to receive the bread from the child.

\section{LESSON XIII}

The Imperfect Indicative Middle and Passive

78. VOCABULARY

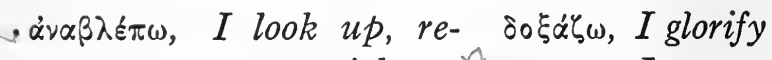
cover sight $\quad \varepsilon_{\pi i \theta \omega,}$ I persuade

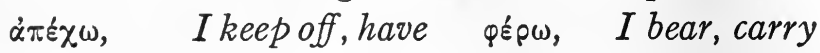
in full (of re- ává, prep., on, upon, ceipts); Midd. along; used with I keep myself accusative only $\dot{\alpha} \pi \circ \theta \nu \dot{\gamma} \sigma x \omega, I$ die from, abstain. in New Testa-

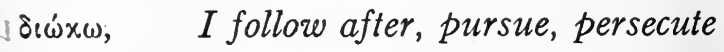

79. The personal endings in the secondary ${ }^{1}$ tenses of the indicative middle and passive are:

Singular

I. $-\mu \eta \eta, I$

2. $-\sigma 0$, thou (you)

3. $-\tau 0$, he, etc.
Plural

$-\mu \varepsilon \theta \alpha$, we

$-\sigma \theta \varepsilon, \quad y e($ you $)$

$-\nu \tau 0$, they

1 The terms "primary" and "secondary" apply to the indicative mode only. 
IMPF. IND. MIDD. AND PASS.

80. The conjugation of $\lambda \hat{u} \omega$ in the imperfect indicative middle is:

\section{Singular}

Plural

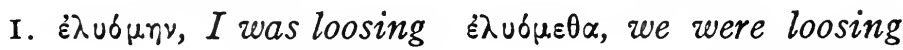
(for) myself (for) ourselves

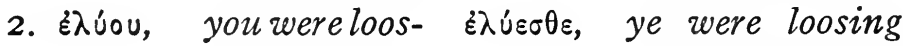
ing (for) (for) yourselves yourself

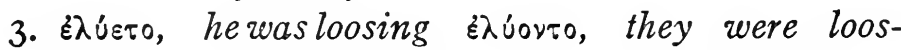
(for) himself ing (for) themselves

a. In the second pers. sing., Ėuvou, -ou is for $-\varepsilon \sigma 0$; intersonantic $\sigma$ drops out and $\varepsilon \circ$ contract to ou.

81. Observe: I. The thematic vowel $\%$. 2. The augment. 3. The accent in first pers. sing.

82. As in the present tense, so also in the imperfect, the middle and passive voices are alike in form.

83. The conjugation of $\lambda \dot{v} \omega$ in the imperfect indicative passive is:

Singular

Plural

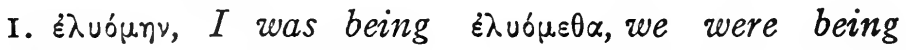
loosed

loosed

2. Ė่úou, you were be- $\varepsilon \lambda{ }^{\prime} \varepsilon \sigma \theta \varepsilon$, you were being ing loosed

loosed

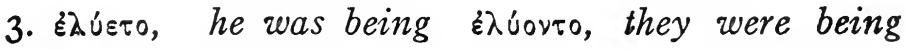
loosed loosed

84. Review the present indicative active (13), middle (53), and passive (59), and the imperfect indicative active (67). 
85. The personal endings of the verb have to express

I. The person of the verb.

2. The number of the verb.

3. The voice of the verb.

In fact they express everything that has to do with the subject.

4. In the indicative they tell whether primary or secondary.

The personal endings of the verb must be mastered. The importance of the forms of the verb cannot be overstressed. If the verb-forms thus far given are thoroughly learned, the student has overcome the greater part of the difficulty in the regular verb.

86. $i \pi \delta$ (prep.) is used with the ablative case to denote the agent, by, especially with the passive

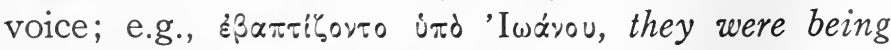
baptized by John.

87.

EXERCISES

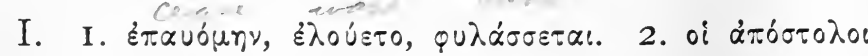

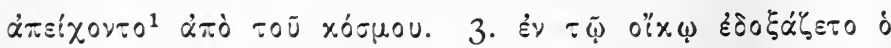

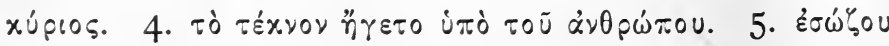
$x \alpha i \dot{\alpha} \vee \varepsilon \dot{\beta} \lambda \varepsilon \pi \varepsilon \varepsilon$.

II. I. The word was being preached by the apostles. 2. The children were washing themselves. 3. I was being judged by men. 4. The angel of the Lord was being heard. 5. The law was being taught in the temple. 6. The servants were looking up into the heavens.

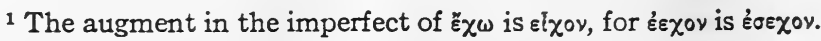

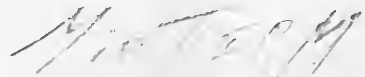




\section{LESSON XIV}

\section{The First Declension or Declension of $\alpha$ - Stems}

\section{8.}

\section{VOCABULARY}

ápxń, beginning

rpapn', writing, scripture

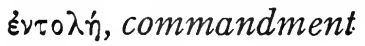

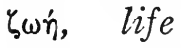

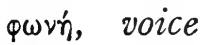

$\psi \cup \times \dot{n}$, soul $\dot{\alpha} \gamma \dot{\alpha} \pi \eta, \quad$ love

$\delta\llcorner\alpha \theta \dot{n} \times \eta$, covenant, testament

$\delta เ x \alpha\llcorner\circ \sigma u ́ v \eta$, righteousness Eipńn, peace xúun, village

89. All substantives of the first declension whose nominatives end in $\alpha$ or $\eta$ are feminine in gender.

90. The feminine article $(\dot{\eta})$ and nominatives in $-\eta$ are declined as follows:

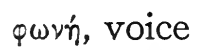

STEM $\varphi \omega \nu \alpha-$

\section{Singular Plural}

Nom. $\dot{\eta} \varphi \omega r \dot{n}$

$$
\alpha i \varphi \omega v \alpha i
$$

Gen. $\tau \tilde{\eta} \varsigma \varphi \omega \nu \tilde{\eta} s \quad \tau \tilde{\omega} \nu \varphi \omega \nu \tilde{\omega} \nu$

Abl. $\tau \tilde{\eta} \varsigma \varphi \omega \nu \tilde{\eta} \varsigma \quad \tau \tilde{\omega} \nu \varphi \omega \nu \tilde{\omega} \nu$

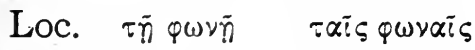

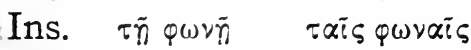

Dat. $\tau \tilde{n} \varphi(\omega) \tilde{n} \quad \tau \alpha i \varsigma \varphi \omega v \alpha i \varsigma$

Acc. $\tau \dot{\eta} \nu \varphi \omega \nu \dot{\eta} \nu \tau \dot{\alpha} \varsigma \varphi \omega \nu \alpha \dot{\alpha} \varsigma$

Voc. $\varphi \omega \nu \dot{n}$ $x \omega \dot{\omega} \mu \eta$, village

\section{STEM $x \omega \mu \alpha-$}

Singular Plural $x \dot{\omega} \mu \eta \quad x \tilde{\omega} \mu \alpha t$ $x \dot{\omega} \mu \eta \bar{s} \quad x \omega \mu \tilde{\omega} \nu$ $x \omega \dot{\omega} \mu \eta \bar{s} \quad x \omega \mu \tilde{\omega} \nu$ $x \dot{\omega} \mu n \quad x \dot{\omega} \mu \alpha i s$ $x \dot{\omega} \mu n \quad x \dot{\omega} \mu \alpha t s$ $x \dot{\omega} \mu n \quad x \dot{\omega} \mu \alpha i s$ $x \dot{\omega} \mu \eta \eta$ $x \dot{\omega} \mu \eta \quad \quad x \tilde{\omega} \mu \alpha \iota$

91. Observe: I. The stem ends in $\alpha$, and is therefore called the $\alpha$-declension. 2. Iota-subscript is always written in the loc., ins., and dat. singular (see $25 a$ ). 
3. When the nominative sing. ends in $-\eta$ the $\eta$ is retained throughout the singular. 4 . In the nominative plural $-\alpha \iota$ is considered short in determining place and kind of accent.

92. The ending - ass in the loc., ins., and dat. plural is a new formation on the analogy of -ors in o-stems. See $25 c$.

93. Nouns that have an acute accent on the last syllable (ultima) are called oxytones (sharp tones); e.g., $\varphi \omega \nu \eta$, $\omega \dot{\eta}$. Learn that all oxytones of the first and second declension have the circumflex accent over the ultima in all the genitives, ablatives, locatives, instrumentals, and datives, See 40.

94. The gen. and abl. plu. of substantives of the first or $\alpha$-declension always have the circumflex accent on the ultima, no matter where the accent is in the nominative singular, since $-\bar{\omega} \nu$ is contracted from

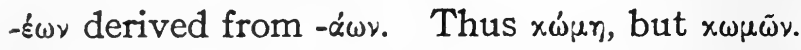

95. Compare the feminine article $(\dot{\eta})$ with the endings of $\varphi \omega r \eta$. Notice that, as in the case of the forms $\delta$ and $o i$ of the masculine article, the forms $\dot{\eta}$ and $\alpha$ i do not have an accent. These forms of the article are called proclitic: they are to be pronounced as a part of the following word.

96.

\section{EXERCISES}

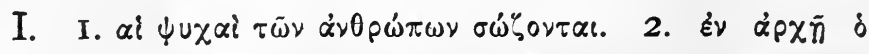

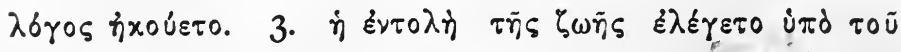

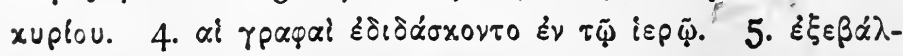

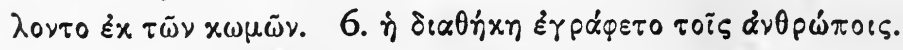


II. I. From the beginning, in the village. (2, $\mathrm{He}$ is teaching the scriptures. 3. We have peace in the soul. 4. The sons were receiving the testament. 5. The apostles heard the commandment from the Lord. 6. The soul is being saved and finds peace and righteousness.

\section{LESSON XV}

First Declension (Continued)

97. VOCABULARY

$\dot{\alpha} \mu \alpha \rho \tau i \alpha, \dot{\eta}, \sin$

$\beta \alpha \sigma \imath \lambda \varepsilon i \alpha, \dot{\eta}$, kingdom

$\dot{E} \times \times \lambda \eta \nabla i \alpha, \dot{\eta}$, assembly, (church)

$\dot{\varepsilon} \xi \circ \cup \sigma i \alpha$, $\dot{\eta}$, authority, power

$\dot{\eta} \mu \varepsilon \dot{\rho} \rho, \dot{\eta}, \quad d a y$ $x \alpha \rho \delta i \alpha, \dot{\eta}$, heart бopi $\alpha, \dot{\eta}, \quad$ wisdom

$\dot{\alpha} \lambda \dot{n} \theta \varepsilon \iota \alpha, \dot{\eta}$, truth $\dot{\alpha} \sigma \theta \varepsilon \dot{v \varepsilon \iota} \alpha, \dot{\eta}$, weakness $\mu \dot{\alpha} x \alpha \iota \rho \alpha, \dot{\eta}$, sword

98. I. As we have seen, a substantive in Greek has case, number, and gender.

2. There are three genders: masculine, feminine and neuter.

99. Nearly all substantives of the second declension with nominatives in -os are masculine; ${ }^{1}$ and all substantives of the second declension with nominatives in -ov are neuter.

100. All substantives of the first declension with nominatives in $-\eta$ or $-\alpha$ are feminine. Those with

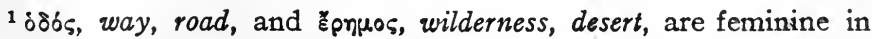
gender.
} 
nominatives in $-\eta \varsigma$ or $-\alpha \varsigma$ are masculine. See Lesson XVI.

But the gender of substantives must often be learned by observation. Hereafter in this book the gender will be indicated in the vocabulary by the article placed after the substantive. (This method is used by most lexicons.) The masculine article, $\dot{0}$, indicates masculine gender; the feminine article, $\dot{\eta}$, feminine gender; the neuter article, $\tau$ ó, neuter gender.

101. Substantives of the first declension with nominatives in $-\alpha$ (preceded by $\varepsilon$,, , or $\rho$ ) are declined as follows:

$x \alpha \rho \delta i \alpha$, heart

STEM $x \alpha \rho \delta\llcorner\alpha-$

\section{Singular}

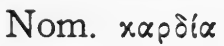

Gen. xapoias

Abl. xapdias

Loc. $x \alpha p \delta i \alpha$

Ins. $x \alpha \rho \delta i \alpha$

Dat. $\quad x \alpha \rho \delta i \alpha$

Acc. $x \alpha p \delta i \alpha \nu$

Voc. $x \alpha \rho \delta i \alpha$
Plural xapoía

$x \alpha \rho \delta ! \tilde{\omega} \nu$

$x \alpha \rho \delta เ \tilde{\omega} \nu$

xapoials

xapdiats

xapoials

$x \alpha p \delta i \alpha s$

$x \alpha p \delta i a$, $\dot{\alpha} \lambda \dot{n} \theta \varepsilon เ \alpha$, truth

STEM $\alpha \lambda \eta \theta \varepsilon \iota \alpha-$

Singular

Plural

$\alpha \lambda \hat{n} \theta=\iota \alpha$

$\alpha \lambda \dot{n} \theta \varepsilon \iota \iota$

$\dot{\alpha} \lambda \eta \theta$ sias

$\dot{\alpha} \lambda \eta \theta \varepsilon เ \tilde{\omega} \nu$

$\alpha \lambda \lambda \theta \theta \varepsilon i \alpha \varsigma$

$\dot{\alpha} \lambda \eta \theta \varepsilon เ \tilde{\omega} \nu$

$\alpha \lambda \eta \theta \varepsilon i \dot{\alpha}$.

$\alpha \lambda \eta \theta \varepsilon i \alpha i s$

$\alpha \lambda \gamma \theta \varepsilon i \dot{\alpha}$.

$\alpha \lambda \eta \theta \theta i \alpha i s$

$\dot{\alpha} \lambda \eta \theta \varepsilon i \dot{\alpha}$.

$\alpha \lambda \eta \theta$ siais

$\alpha \lambda \lambda \dot{\theta} \theta \varepsilon \iota \alpha$

$\alpha \lambda \eta \theta \varepsilon i \alpha \varsigma$

$\alpha \lambda \dot{n} \theta \varepsilon \ll \alpha$

$\dot{\alpha} \lambda \dot{n} \theta \varepsilon \ll \iota$

102. Observe: I. When $\varepsilon$, ', or $p$ precedes $-\alpha$ in the nominative singular, the $\alpha$ is retained throughout the singular. ${ }^{1}$ 2. The $-\alpha$ after $\varepsilon$, !, or $p$ may be long as in $x \alpha \rho \delta^{\prime} i \alpha$ or short as in $\alpha \lambda \dot{n}^{\prime} \theta \varepsilon \iota \alpha .3$ When the $-\alpha$ is short (in the first declension) in the nominative singular,

${ }^{1}$ Sometimes $-\eta \varsigma$ is found in the gen. and abl. sing. after $\varepsilon$, $\iota$, or p. 
it is also short in the accusative singular. But in the accusative plural the $-\alpha$ is long in the first declension. 4. In the gen., ab1., loc., ins., and dat. singular of $\alpha \lambda \dot{r} \theta \varepsilon \varepsilon \alpha$ the accent is brought forward to the penult because the ultima is long. Cf. 33,2 , and 26, I.

103.

\section{EXERCISES}

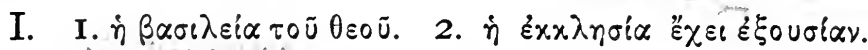

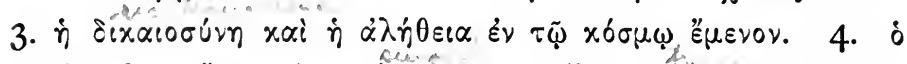

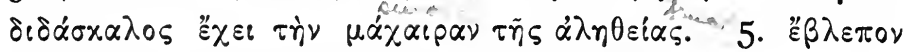

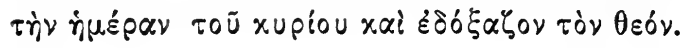

II. I. In the hearts of men. 2. Wisdom was being taught in the church. 3. It is being written in truth. 4. The sword of truth pursues sin. 5. The Lord has power to save men.

\section{LESSON XVI}

\section{First Declension (Continued)}

104.

$\gamma \lambda \tilde{\omega} \sigma \sigma \alpha, \dot{\eta}, \quad$ tongue

$\delta \delta \xi \alpha, \dot{\eta}, \quad$ glory

$\theta \alpha \dot{\lambda} \alpha \sigma \sigma \alpha, \dot{\eta}, \quad$ sea

$\mu \alpha \theta \eta \tau \dot{\eta} s, \delta$, disciple

$\pi$ poprins, $\delta$, prophet

$\varepsilon \pi \alpha \gamma \gamma \varepsilon \lambda i \alpha, \dot{\eta}$, promise
VOCABULARY

$x \varepsilon \varphi \alpha \lambda \dot{\eta}, \dot{\eta}, \quad h e a d$

oixia, $\dot{\eta}, \quad$ house

$\pi \alpha \rho \alpha \beta \circ \lambda \dot{\eta}, \dot{\eta}$, parable

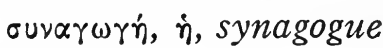

$\chi \propto \rho \alpha \dot{\alpha}, \dot{\eta}, \quad j o y$

๘ँp $, \dot{\eta}, \quad$ hour 
105. Substantives of the first declension with nominatives in $-\alpha$, not after $\varepsilon$, 1 , or $\rho$, are declined as follows:

\section{STEM $\gamma \lambda \omega \sigma \sigma \alpha-$}

\section{Singular}

Nom. $\quad \gamma \lambda \tilde{\omega} \sigma \sigma \alpha$

Gen. $\quad \gamma \lambda \omega \operatorname{sons}$

Abl. $\quad \gamma \lambda \omega ́ s o n s$

Loc. $\quad \gamma \lambda \omega \dot{\omega} \sigma 0 n$

Ins. $\quad \gamma \lambda \omega \sigma \sigma n$

Dat. $\quad \gamma \lambda \omega \sigma o n$

Acc. $\quad \gamma \lambda \bar{\omega} \sigma \sigma \alpha \nu$

Voc. $\quad \gamma \lambda \bar{\omega} \sigma \sigma \alpha$
Plural

$\gamma \lambda \bar{\omega} \sigma \sigma \alpha i$

$\gamma \lambda \omega \sigma \sigma \tilde{\omega} y$

$\gamma \lambda \omega \sigma \sigma \tilde{\omega} \nu$

$\gamma \lambda \omega \dot{\omega} \sigma \alpha i s$

$\gamma \lambda \omega \dot{\sigma \sigma \alpha l s}$

$\gamma \lambda \omega \dot{\sigma \sigma \alpha a l s}$

$\gamma \lambda \omega \sigma \sigma \alpha \varsigma$

$\gamma \lambda \bar{\omega} \sigma \sigma \alpha !$

106. Observe: I. When $\varepsilon$, , or $\rho$ does not precede $\alpha$ of the nom. sing., the $\alpha$ is changed to $\eta$ in the gen., ab1., loc., ins., and dat. sing. 2. When the $\alpha$ is short in the nom. sing. it is also short in the acc. sing.

107. Contract substantives of the first declension as $\gamma \tilde{\eta}, \dot{\eta}$, earth, and $\mu \nu \tilde{\alpha}, \dot{\eta}$, mina (a weight and sum of money) are declined like $\varphi \omega \nu \dot{n}$ and $x \alpha \rho \delta i \alpha$ respectively, except that they have the circumflex accent on the ultima throughout.

108. Substantives of the first declension with nom. sing. in $-\eta \varsigma$ or $-\alpha \varsigma$ are masculine in gender. See Ioo.

109. The declension of $\pi$ poprirns, $\delta$, prophet; is 


\section{THE FIRST DECLENSION}

\section{STEM $\pi p \circ p \eta \tau \alpha-$}

\section{Singular}

Nom. $\pi$ popinins

Gen. $\quad \pi p 0 p$ nं 000

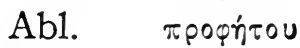

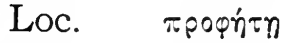

Ins. $\quad \pi$ popinn

Dat. $\quad \pi p o p r i n$

Acc. $\quad \pi p o p$ ring

Voc. $\pi \rho \circ p \tilde{\eta} \tau \alpha$

\section{Plural}

$\pi \rho \circ \tilde{\eta} \tau \alpha \ell$

$\pi \rho \circ p \eta \tau \tilde{\omega} \nu$

$\pi \rho \circ p \eta \tau \bar{\omega} \nu$

$\pi$ popritals

$\pi \rho o p \dot{r} \tau \alpha s$

$\pi \rho o p \dot{i} \tau \alpha \cdot s$

$\pi \rho \circ \varphi \hat{r} \tau \alpha \varsigma$

$\pi \rho \circ p \tilde{r} \tau \alpha \iota$

110. Observe: I. The gen. and abl. sing. -ou is the same as in the o-declension. 2. The voc. sing. is $-\alpha$. Masculine substantives of the first declension in - $\tau$. have $-\alpha$ in the vocative sing. 3. The plurals of all substantives of the $\alpha$-declension are alike.

111. The singular of veavias, $\delta$, youth, is

Nom. veavias,

Loc. veavía,

Acc. $\nu \varepsilon \alpha v^{\prime} \alpha \nu$,
Gen. veaviou,

Ins. $v \varepsilon \alpha v^{\prime} \alpha$,

Voc. veavía.

Abl. veavíou,

Dat. veavía,

\section{2.}

\section{EXERCISES}

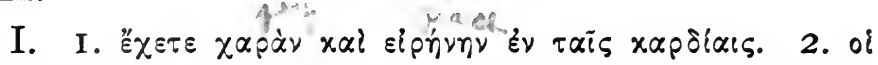

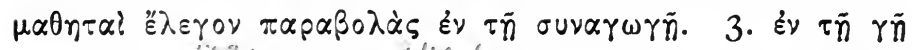

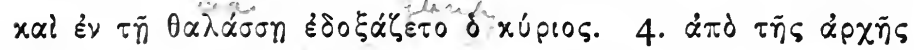

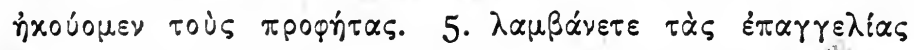

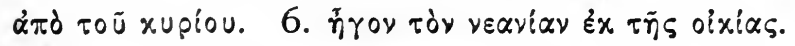

II. I. They were remaining in the house. 2. Righteousness and love remain in the world. 3. The hour of the Lord is announced. 4. The prophets are teaching the disciples in parables. 5. The promises were spoken from the beginning. 


\section{LESSON XVII}

\section{Adjectives of the First and Second Declension}

\section{3.}

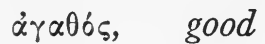

$\dot{\alpha} \gamma \alpha \pi \eta \tau \dot{s} \varsigma$, beloved

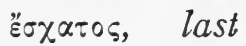

\%axós, evil, bad
VOCABULARY

xàós, good, beautiful

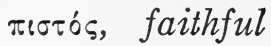

$\pi \rho \tilde{\omega} \tau \circ$ s, first

114. Most adjectives of the vowel (first and second) declension have three endings, -os (masc.), $-\eta$ or $-\alpha$ (fem.), -ov (neut.). Adjectives are declined in gender, number and case.

115. The adjective $\dot{\alpha} \gamma \alpha \theta$ ós is declined as follows:

Singular

Plural

Masc. Fem. Neut. Masc. Fem. Neut.

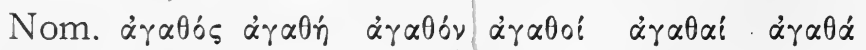

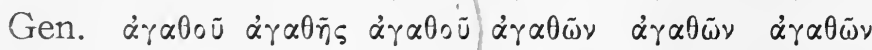
Abl. $\dot{\alpha} \gamma \alpha \theta 0 \tilde{u} \dot{\alpha} \gamma \alpha \theta \tilde{\eta} s \quad \dot{\alpha} \gamma \alpha \theta 0 \tilde{u} \dot{\alpha} \gamma \alpha \theta \tilde{\omega} \nu \quad \dot{\gamma} \gamma \alpha \theta \tilde{\omega} \nu \quad \dot{\alpha} \gamma \alpha \theta \tilde{\omega} \nu$ Loc. $\quad \dot{\alpha} \gamma \alpha \theta \tilde{\varphi} \quad \dot{\alpha} \gamma \alpha \theta \tilde{n} \quad \dot{\alpha} \gamma \alpha \theta \tilde{\omega} \quad \dot{\alpha} \gamma \alpha \theta 0 \tilde{i} \varsigma \quad \dot{\alpha} \gamma \alpha \theta \alpha \bar{i} \varsigma \quad \dot{\alpha} \gamma \alpha \theta_{0} i \bar{s}$

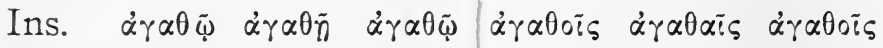
Dat. $\dot{\alpha} \gamma \alpha \theta \tilde{\varphi} \quad \dot{\alpha} \gamma \alpha \theta \tilde{n} \quad \dot{\alpha} \gamma \alpha \theta \tilde{\varphi} \quad \dot{\alpha} \gamma \alpha \theta 0 \tilde{s} s \quad \dot{\alpha} \gamma \alpha \theta \alpha \tilde{\iota} \varsigma \quad \dot{\alpha} \gamma \alpha \theta 0 \tilde{c}$

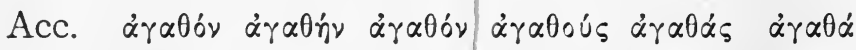

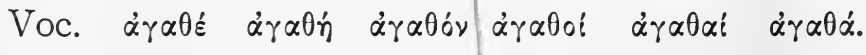

116. Observe: I. In form the masc. is declined exactly like a masculine substantive of the second declension (see oupavós, 39); the neuter like a neuter substantive of the second declension (see हैprov, 42); and the feminine like a feminine substantive in $-\eta$ (see $\varphi \omega \nu \dot{n}, 90$ ). 
a. The accent of the feminine genitive and ablative plural does not follow the accent of the feminine substantive of the $\alpha$-declension (given in 94), but the regular accent of the masculine: thus the genitive and ablative plural feminine form of है $\sigma \chi \alpha \tau \circ \varsigma$ is $\dot{\varepsilon} \sigma \chi \alpha \dot{\alpha} \tau \omega \nu$.

117. Adjectives agree with the substantives which they modify, in gender, number, and case; e.g.,

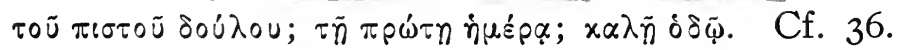

118. Adjectives are used tc refer to substantives in two ways, either (I) as an attribute or (2) as a predicate.

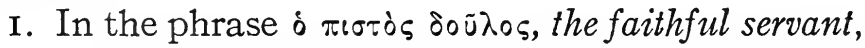
$\pi \imath \sigma o^{\prime}$, faithful, is an attribute adjective; it qualifies

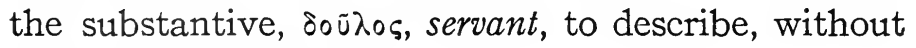
any assertion about it.

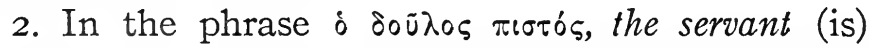
faithful, the predicate adjective $\pi \iota \sigma \delta s$, faithful, makes an assertion about the substantive $\delta \circ \tilde{u} \lambda \circ$ s, servant.

It is important to understand this distinction between the attribute and the predicate adjective in Greek. The distinction lies in just this, that the predicate presents an additional statement, while the attribute is an adherent description.

119. Examples of the positions of the adjective:

I. Attributive position of the adjective-

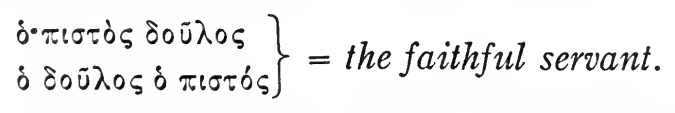

Note that the adjective comes immediately after the article. 
There is another order of the attributive position,

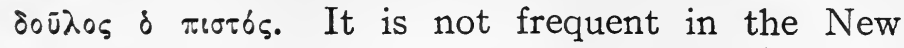
Testament.

2. Predicate position of the adjective-

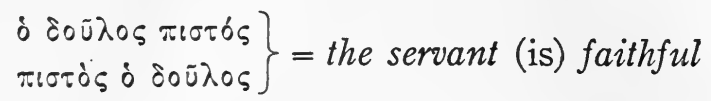

Note that the adjective does not come immediately after the article but either precedes the article or follows the substantive.

120. When the article is not present, the context must decide whether an adjective is attributive or

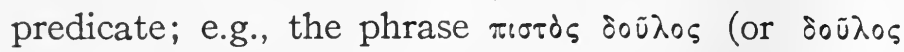
$\pi เ \sigma \tau \delta$ ) may be either attributive, a faithful servant, or predicate, a servant (is) faithful.

121. In the New Testament $8 \lambda_{0}$, whole, never has the attributive position.

122.

\section{EXERCISES}

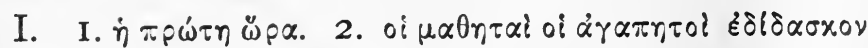

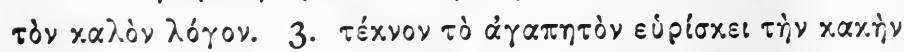

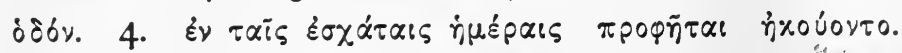

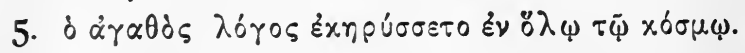

II. I. The evil prophet was not ${ }^{1}$ proclaiming the good promises. (2.) On the last day the disciple was speaking in the synagogue. 3. The whole house was receiving the word of God. 4. In the first hour of the day they were glorifying the Lord.

$$
1 \text { See } 130 .
$$




\section{LESSON XVIII}

Adjectives of the First and Second Declension (Continued)

123.

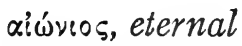

$\delta i x \alpha 10 s$, righteous

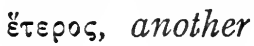

¿810s, one's own
VOCABULARY

$$
\begin{aligned}
& \text { rovos, only, alone } \\
& \text { uıxpós, small, little } \\
& \text { Tounpos, evil }
\end{aligned}
$$

124. Learn the declension of ' $\delta 105$, one's own, and uıxpós, small, in $\S \mathrm{B} \mathrm{I} 3$.

Observe: I. When $\varepsilon$, ', or $\rho$ precedes the final vowel of the stem, the feminine has $-\alpha$ in the nominative sing.

2. In the nom. and gen. plur. fem. the accent follows the masc.

125. Some adjectives (especially compounds) have only two endings, the masc. and fem. having the

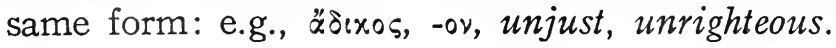

126. Prepositional phrases or adverbs are often used like adjectives in the attributive position:

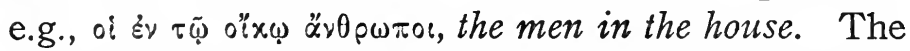

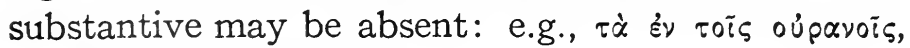
the things in the heavens.

127. The adjective in any gender without a substantive is often used as a practical substantive, usually with the article, but not always: e.g., oi $x \alpha \lambda_{\text {of }}$, the good (men or people); to $\alpha$ ratóv, the good thing;

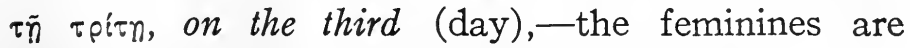
usually examples of ellipsis of $i \mu \varepsilon \varepsilon^{\prime} \rho \alpha$, $\delta \delta \delta$, etc. 
128. As a complement, the infinitive (mostly in the active voice) is used with adjectives, substantives, and verbs that imply power or ability, fitness,

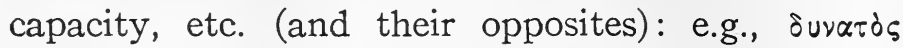

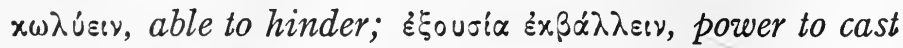

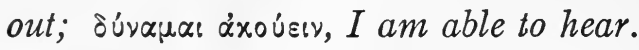

129.

EXERCISES

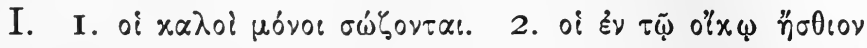

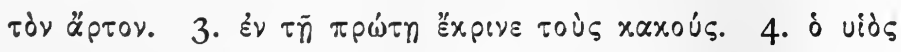

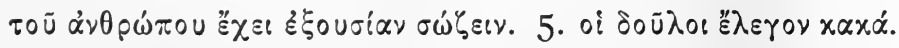

II. I. On the first day he was preaching in the synagogue. 2. The first, last; the last, first. 3. The faithful are saved. 4. He has power to cast out the evil. 5. The men in the boat know the sea.

\section{LESSON XIX}

Personal Pronouns and siui, I am

130. VOCABULARY

$\dot{\alpha} \lambda \lambda \dot{\alpha}$, adversative conj., but ráp, co-ordinating conj., for

$\gamma \hat{\varepsilon}, \quad$ enclitic postpositive particle ย̌ Eipi, I am giving special prominence to

a word, indeed, at least

$\delta \dot{\varepsilon}$, copulative and adversative (milder than $\alpha \lambda \lambda \dot{\alpha}$ ) conj., in the next place, and; but, on the other hand.

$$
\left.\begin{array}{l}
\text { oú } \\
\text { oúx } \\
\text { oux }
\end{array}\right\} \text { not }
$$


$a$. Words that cannot come first in a sentence are called postpositives. $\gamma \dot{\alpha} p, \gamma \dot{\varepsilon}$, and $\delta \dot{\varepsilon}$ are postpositives. $b$. ou is written before consonants; $o \dot{u} x$ before vowels; oux before the rough breathing.

131. Generally speaking, the pronoun is a word that stands in place of a substantive. The idea that is set forth by a pronoun is the relation of a subject or object to the speaker. The reason for the use of the pronoun, then, is to avoid the repetition of the substantive.

132. The declension of the first personal pronoun

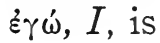

\section{Singular}

Nom. èr

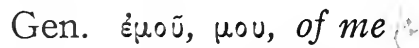

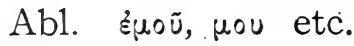

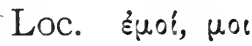

Ins. Époi, $\mu \circ$

Dat. É $\mu o t, \mu o \varepsilon$

Acc. Ėนé, $\mu \varepsilon$
Plural insis, we $\dot{\eta} \mu \tilde{\omega} \nu$, of $u s$ $\dot{\eta} \mu \tilde{\omega} \nu$, etc. $\dot{\eta} \mu i \nu$ $\dot{\eta} \mu i \tau$ $\dot{\eta} \mu \tilde{\tau}$ $\dot{\eta} \mu \tilde{\alpha} \varsigma$

133. The declension of the second personal pronoun, oú, thou, is

Singular

Nom. oú,

Gen. coũ, oou, of thee

Abl. $00 \tilde{u}$, oou etc.

Loc. coí, ool,

Ins. ooi, oor

Dat. ooi, oci

Acc. $\sigma \varepsilon, \sigma \varepsilon$.
Plural ipeis, ye (you)

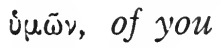
i $\mu \tilde{\omega} \nu$ etc.

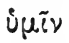

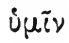
ineiv $i \mu \tilde{\alpha} \varsigma$ 
134. Observe: In the singular of the first and second personal pronouns there are, except in the nominative, two forms for each case, an accented form and an unaccented form (which in the first person is also shorter than the accented form). These forms are called enclitics (see I38).

135. I. Commonly the accented or emphatic forms are used when emphasis or contrast is desired. Yet it is not certain that all emphasis is absent when the unaccented or enclitic forms are used.

2. With prepositions the emphatic or accented forms are used generally, except with $\pi \rho b s$, which ordinarily has $\pi$ pós $\mu \varepsilon$.

3. In general the personal pronouns were not used in the nominative case unless emphasis or contrast

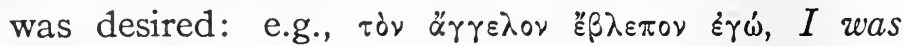
looking at the angel (It was $I$ who was looking at the angel). This follows from the fact that the verb uses the personal pronouns as personal endings (as explained in 17 ), and no need was felt for the separate expression of the personal pronoun in the nominative.

136. The conjugation of the present indicative of eipi, I am, is as follows:

Singular

I. eipl, I am

2. Ei, thou art

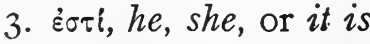

Plural

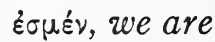
દorर́, ye (you) are Eioi, they are

Present infinitive giva, to be

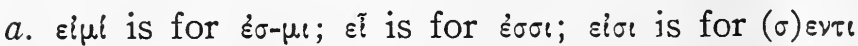
for $(h) \varepsilon \nu \tau \imath$; $\varepsilon^{i} \nu \alpha \iota$ is probably for $\varepsilon^{\prime} \sigma-\nu \alpha \iota$. $b$. All 
the forms of the present indicative of ei $\mu$, except $\varepsilon$, are enclitic (see 138). c. It was noted in 18 that the primary act. end. $-\mu$, was dropped, and the preceding $\circ$ lengthened to $\omega$. Some verbs retain this $-\mu$ and do not have the thematic $\%$. The former make up what is called the $\omega$ - conjugaticil, the latter the $\mu$ - conjugation. To the latter belongs ei $\mu l$.

137. When the verb eipl is used merely as a connective or copula, it has the predicate nominative:

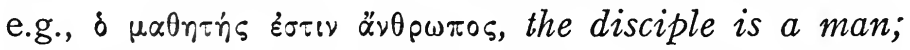
see examples below under $138, \mathrm{I}-5$. Note that the subject may be known from the predicate whenever the subject has the article and the predicate does

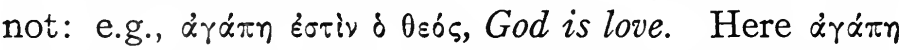
is the predicate because it does not have the article, while $\theta$ rós does have the article.

138. Enclitics are words attaching themselves so closely to the preceding word as to be pronounced with it. Usually they have no accents of their own.

The word before an enclitic is treated as follows:

I. If the preceding word has an acute accent on the antepenult, it receives an additional accent (acute) on its ultima from any enclitic, whether of one syllable (monosyllabic) or of two syllables (dissyllabic):

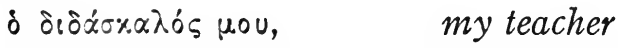

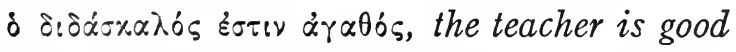

2. If the preceding word has an acute accent on the penult, its accent is not affected in any way: 
then a monosyllabic enclitic loses its accent; but a dissyllabic enclitic retains its accent:

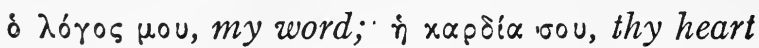

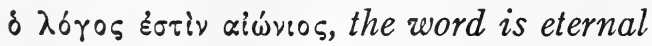

3. If the preceding word naturally has an acute accent on the ultima, it keeps its own accent, and any enclitic loses its accent.

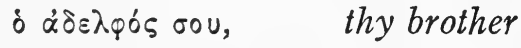

oi $\alpha \delta \varepsilon \lambda \varphi \circ i$ siol $\pi \imath \tau \tau o l$, the brothers are faithful

4. If the preceding word has a circumflex accent on the penult, it receives an additional accent (acute) on its ultima from any enclitic:

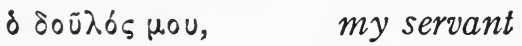

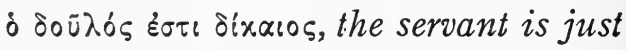

5. If the preceding word has a circumflex accent on the ultima, its accent is not affected in any way, and any enclitic loses its accent:

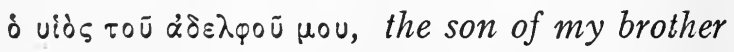

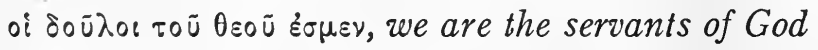

139. Observe: I. A monosyllabic enclitic regularly loses its accent. 2. A dissyllabic enclitic retains its accent only under the condition named in 2 above.

140. An enclitic sometimes retains its accent:

I. When there is emphasis on the enclitic or when the enclitic begins a sentence.

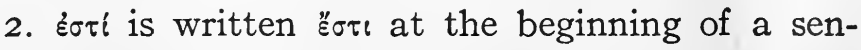
tence, when it means exist or is possible, and when it

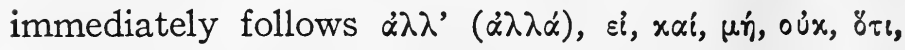

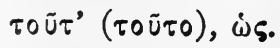


141. A proclitic (see note to 72 and 95) or an enclitic followed by an enclitic receives an acute accent: e.g. ö $\gamma \varepsilon \delta 0 \tilde{\nu} \lambda \dot{s} \varsigma \mu \circ \dot{\varepsilon} \delta \tau \tau$.

(But under I38-I40 modern critics and editors differ.)

142.

EXERCISES

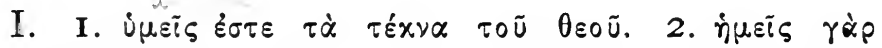

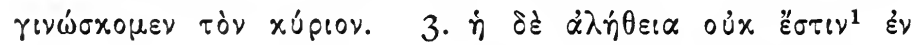

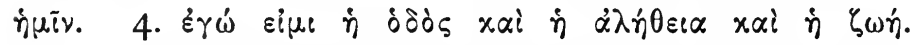

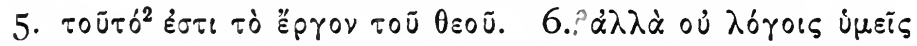
$\sigma \omega \dot{\zeta} \zeta \varepsilon \sigma \theta \varepsilon$.

II. I. My house is in the village. 2. We are the servants of the Lord. 3. The way is bad, but you know me. 4. You are a prophet, for from you are sent forth words of wisdom. 5. We have bread for you (sing.). 6. You are my disciples.

\section{LESSON XX}

Third Personal Pronoun. Imperfect Ind. of eipl 143.

VOCABULARY

$\ddot{\alpha} \lambda \lambda \circ \varsigma,-\eta,-0$, other

в!, conj., if

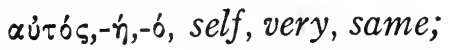

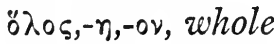

$$
\begin{aligned}
& \text { he, she, it } \text { örl, conj., because, that } \\
& \text { oxorí, } \dot{\eta}, \text { darkness }
\end{aligned}
$$

1 Certain words, i.e. words ending in - $\sigma$, the third personal sing. of past tenses (in $-\varepsilon$ ), and é $\sigma i$, may add $\nu$. This is called movable $v$. Movable $\nu$ in the older Greek was written when it would be followed by a word beginning with a vowel; but later it was written

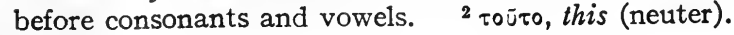


144. The declension of aurós is as follows:

Singular

\begin{tabular}{|c|c|c|c|}
\hline Nom. & $\begin{array}{l}\text { Masc. } \\
\text { aúrós }\end{array}$ & 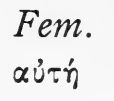 & $\begin{array}{l}\text { Neut. } \\
\text { aúró }\end{array}$ \\
\hline Gen. & $\alpha \dot{U} \tau 0 \tilde{U}$ & $\alpha \dot{U} \tau \tilde{\eta} S$ & $\alpha \dot{U} \tau \circ \tilde{u}$ \\
\hline Abl. & $\alpha \dot{U} \tau 0 \tilde{U}$ & $\alpha \dot{U} \tau \tilde{\eta} s$ & $\alpha \dot{U} \tau 0 \tilde{U}$ \\
\hline Loc. & $\alpha \dot{\nu} \tau \bar{\varphi}$ & $\alpha \dot{u}=\bar{n}$ & $\alpha \dot{U} \div \bar{\varphi}$ \\
\hline Ins. & $\alpha \dot{u} \tau \bar{\varphi}$ & $\alpha \dot{u}=\tilde{n}$ & $\alpha \dot{U} \tau \tilde{\varphi}$ \\
\hline Dat. & $\alpha \dot{U} \tau \bar{\varphi}$ & $\alpha \dot{u}=\tilde{n}$ & $\alpha \dot{U} \tau \bar{\varphi}$ \\
\hline Acc. & 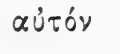 & $\alpha \dot{u} \tau \dot{n} \nu$ & $\alpha u ̉ \tau o ́$ \\
\hline \multicolumn{4}{|c|}{ Plural } \\
\hline Nom. & 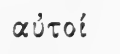 & $\alpha \dot{U} \tau \alpha i$ & $\alpha \dot{\nu} \tau \alpha^{\prime}$ \\
\hline Gen. & $\alpha \dot{u} \tau \tilde{\omega} \nu$ & $\alpha \dot{U} \tau \tilde{\omega} \nu$ & $\alpha \dot{\tau} \tau i$ \\
\hline Abl. & $\alpha \dot{u} \tau \tilde{\omega} \nu$ & $\alpha \dot{u} \tau \tilde{\omega} \nu$ & $\alpha \dot{u} \tau \bar{u}$ \\
\hline Loc. & aúrois & 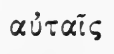 & Q'ं $\tau 0$ \\
\hline Ins. & aủroĩs & $\alpha \dot{u} \tau \alpha \tilde{\imath} \varsigma$ & $\alpha u ̉ \tau 0$ \\
\hline Dat. & $\alpha \dot{v} \tau 0 \tilde{s}$ & $\alpha \dot{u} \tau \alpha i \bar{s}$ & $\alpha \cup \dot{0}=0$ \\
\hline Acc. & aủzoús & $\alpha \dot{u}=\alpha^{\prime} s$ & $\alpha \dot{u} \approx \alpha^{\prime}$ \\
\hline
\end{tabular}

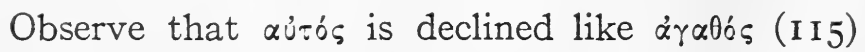
except that $\alpha \dot{u}$ rós has no vocative and the neuter nom. and acc. sing. have no $-v$.

145. Meaning and uses of aútós. It is properly a demonstrative.

I. As an intensive pronoun aurós means self; himself, herself, itself, etc.; and is in the predicate position ( I I 9, 2):

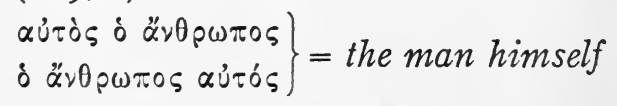

2. As an identical pronoun aurós means same, and is in the attributive position (I $8, \mathbf{I}$ ):

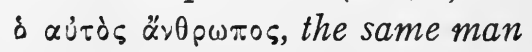


When the article precedes aũbs, the meaning is always the same.

3. When used alone in the genitive, ablative, locative, instrumental, dative, and accusative cases (the "oblique" cases), this word is the simple personal pronoun of the third person:

$\beta \lambda \varepsilon ́ \pi \omega$ aủróv, I see him $\pi \hat{\varepsilon} \mu \pi \circ \mu \varepsilon \nu$ aúroús, we send them

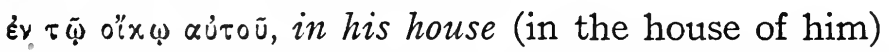

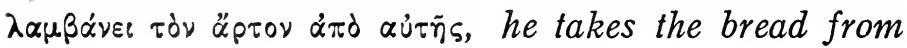
her

(With aũós in the nominative, sometimes it is not clear whether we have simply an emphatic "he," etc., or an intensive "self.")

146. I. The substantive to which a pronoun refers is called its antecedent:

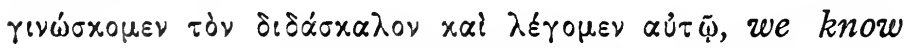
the teacher and speak to him.

$\tau \delta \nu \delta \iota \delta \dot{\alpha} \sigma x \alpha \lambda_{0 \nu}$ is the antecedent of $\alpha \dot{\tau} \tau \tilde{\varphi}$

2. A pronoun agrees with its antecedent in gender and number. Cf. $\delta \_\delta \dot{\alpha} \sigma \alpha \lambda \lambda_{0 \nu}$ (masc. gender, sing. number) and $\alpha \dot{\tau} \tau \tilde{\varphi}$ (masc. gender, sing. number).

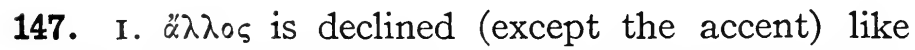
aúrós. Note -0 in the nom. and acc. neuter singular. a $\lambda \lambda{ }_{0}$ is used alone and with the article (but in New Testament never in the senses of "the rest of").

2. $8 \lambda_{0}$ s always has the predicate position in the

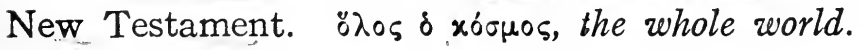


148. The imperfect indicative of sipi, $I \mathrm{am}$, is

Singular

I. ( $\tilde{\eta} \nu)$ and $\ddot{\eta} \mu \eta \nu, I$ was

2. $\tilde{\eta} s$ and $\dot{\eta} \sigma \theta \alpha$, thou wast

3. $\tilde{\eta} \nu$, he was
Plural

$\tilde{\eta} \mu \varepsilon \nu$ and $\ddot{\eta} \mu \varepsilon \theta \alpha$, we were

$\tilde{\eta} \tau \varepsilon$, $\tilde{n} \sigma \alpha \nu$, ye were

they were

a. The middle form $\ddot{n} \mu \eta \nu$ has practically thrust out the active form $\tilde{\eta} \nu . b$. $\tilde{\eta} \sigma \theta \alpha$ is an old perf. form, found twice in New Testament. c. $\eta \mu \varepsilon \theta \alpha$ is found nearly as often as $\eta_{\mu \varepsilon \nu}$ in New Testament.

For the meaning of the imperfect indicative see 65 .

149. Conditional Sentences.

There are four separate forms for Greek conditions. The first is:

\section{The condition determined as fulfilled.}

Here any tense of the indicatiye is used, generally after $\varepsilon$, if, 1 in the protasis (the if-clause). The apodosis (conclusion) generally has the indicative (any tense), but any mode may be used according to what is wanted, e.g.:

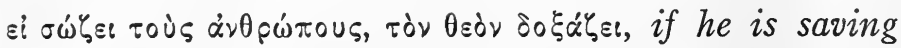
men, he is glorifying God.

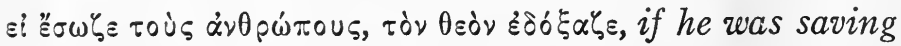
men, he was glorifying God.

"The indicative states the condition as a fact. It may or may not be true in fact. The condition has nothing to do with that, but only with the statement."

The negative of the protasis is generally oú, not. ${ }^{2}$

1 Infrequently $\varepsilon^{\prime} \alpha \nu$, if, is used.

2 A few times $\mu$, not, is found. 
150. EXERCISES

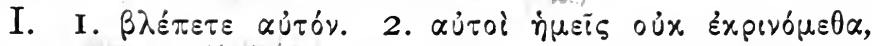

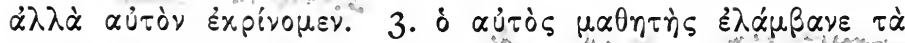

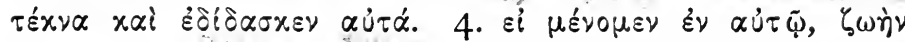

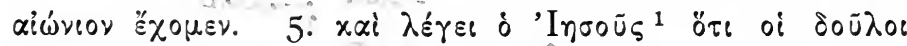

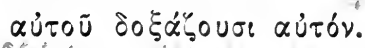

II I. I glorify him. 2. Darkness is not in him. 3. He himself is the life. 4. On ( $\varepsilon v)$ the same day he was teaching them. 5. If we receive him, he saves us. 6. We know the truth and proclaim it. 7. He has other servants in the world. 8. He was in the house. 9. They were faithful men.

\section{LESSON XXI}

\section{Defective ("Deponent") Verbs}

The Demonstrative Pronouns oũzos and Éxeivos

151.

VOCABULARY

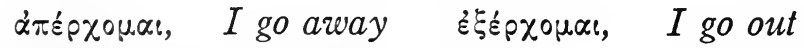

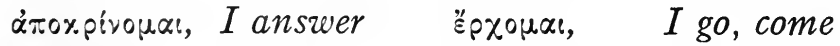

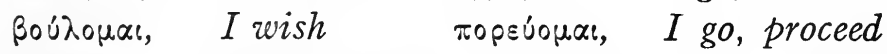

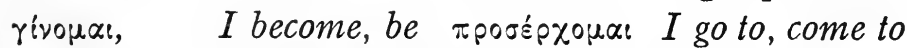

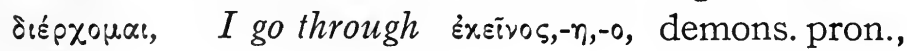
that (one) Súvaual, I amable, can oữos, aütn, demons. pron.

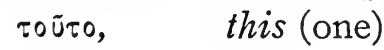

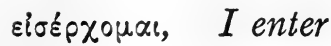

1 Jesus. 
$\delta ı \dot{\alpha}$, prep. (orginally "interval between") with gen. through, by; with acc., because of, for the sake of, on account of.

$\pi$ pós, prep. (originally near, facing) with loc., near, by; with acc., towards, to; with abl. (once), "from the point of view of."

a. Note the many compound ${ }^{1}$ verbs in the vocabulary, especially a preposition + हैp $\propto \rho \mu \alpha$. Many of these verbs occur hundreds of times in the New Testament. $b$. Súvaua does not have a thematic vowel; $\alpha$ appears in all persons. In the second

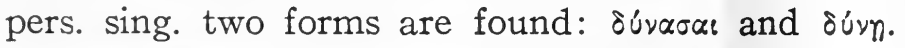
c. yivoual is used also as a copula (see I37).

152. Defective verbs. Some verbs were used in all the voices in all the tenses, as $\lambda \dot{u} \omega$; some verbs in some tenses were used only in one voice and in other tenses, in another voice, as $\beta \alpha i v \omega, I$ go (future $\beta \dot{\eta} \sigma o \mu \alpha$ ) ; some verbs were used in one voice only, as $x \varepsilon i \mu \alpha$, I lie (am laid).

The term defective is applied to those verbs which are used either in the middle voice or in the passive voice and not in the active voice but seemingly have

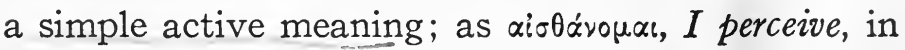

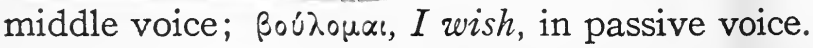

The verbs in the vocabulary (I5I) are defective verbs. But some of these verbs have active forms

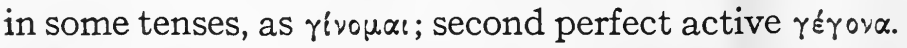

These verbs have been called "deponents" (middle or passive) because it was difficult to see the distinctive force of the voice. Yet it is not hard to 
recognize the personal interest of the subject in the verbs in the middle voice.

153. The declension of oũros is:

\section{Singular}

\begin{tabular}{|c|c|c|c|}
\hline & Masc. & Fem. & Neut. \\
\hline Nom. & OŨสOs & $\alpha u ̈ \tau \eta$ & $\tau \circ \tilde{\tau} \tau 0$ \\
\hline Gen. & 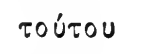 & $\tau \alpha u ́ \tau \eta \varsigma$ & Toútou \\
\hline Abl. & Toútou & $\tau \alpha u ́ \tau \eta s$ & Toútou \\
\hline Loc. & 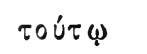 & $\tau \alpha u ́ \tau n$ & $\tau \circ \mathcal{U} \tau \varphi$ \\
\hline Ins. & $\tau 0 \cup ́ \tau \varphi$ & $\tau \alpha u ́ \tau n$ & $\tau \circ u ́ \tau \varphi$ \\
\hline Dat. & $\tau 0$ ப́ $\tau \varphi$ & $\tau \alpha u ́ \tau n$ & $\tau \circ u ̛ \tau \varphi$ \\
\hline Acc. & 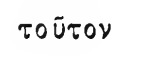 & $\tau \alpha u ́ \tau \eta \nu$ & 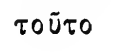 \\
\hline \multicolumn{4}{|c|}{ Plural } \\
\hline & Masc. & Fem. & Neut. \\
\hline Nom. & Oป̃To: & $\alpha \tilde{u} \tau \alpha !$ & $\tau \alpha \tilde{u} \tau \alpha$ \\
\hline Gen. & $\tau \circ u ́ \tau \omega \nu$ & $\tau \circ \cup ́ \tau \omega \nu$ & $\tau \circ u ́ \tau \omega \nu$ \\
\hline $\mathrm{Abl}$ & $\tau 0 u ́ \tau \omega \nu$ & $\tau 0 u ́ \tau \omega \nu$ & $\tau \circ u ́ \tau \omega \nu$ \\
\hline Loc. & Toútols & $\tau \alpha u ́ \tau \alpha \iota \varsigma$ & toútols \\
\hline Ins. & toútois & $\tau \alpha u ́ \tau \alpha i \varsigma$ & 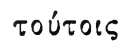 \\
\hline Dat. & 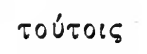 & $\tau \alpha u ́ \tau \alpha \backslash \varsigma$ & toútors \\
\hline Acc. & roúrous & $\tau \alpha u ́ \tau \alpha \varsigma$ & $\tau \alpha \tilde{U} \tau \alpha$ \\
\hline
\end{tabular}

154. Observe: I. The rough breathing occurs in the nom. masc. and fem., sing. and plural, but all other forms begin with $\tau$. 2. The diphthong of the penult, ov or $\alpha v$, varies as the vowel of the ultima, $o(\omega)$ or $\alpha(\eta)$. 3. The accent remains on the penult.

155. The declension of Éxeivos is like that of aúós (except the accent). Note in the neuter sing. nom. and acc. Exxivivo. 
156. oũros and Éxeĩyos are demonstrative pronouns. In contrast, oĩ os refers, as a rule, to what is near or last mentioned, and Éxeivos to what is remote, or absent.

157. Use of oũros and Éxeĩus. I. When they are used with a substantive, they commonly have the

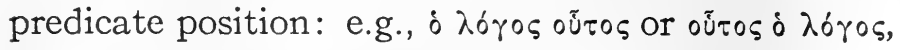

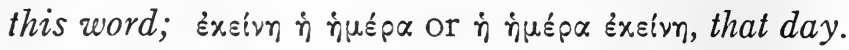

2. When the article does not occur with the substantive, the substantive is in the predicate: e.s.,

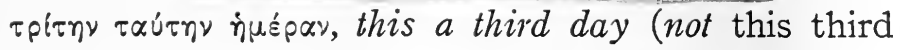
day).

3. They are often used alone, without substantives: e.g., oũros, this one (man or person); Exxivn,

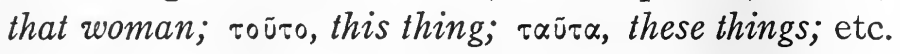

158.

\section{EXERCISES}

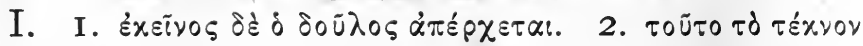

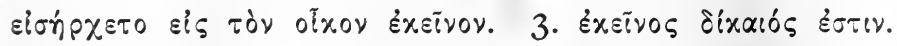

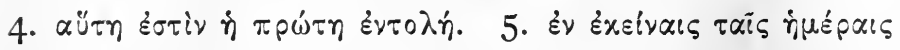

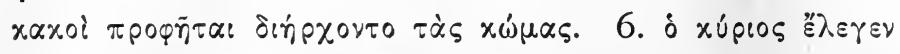

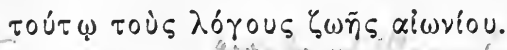

II. I. This world; that gift. 2. This disciple knows the law and the prophets. 3. This is the work of God. 4. Those children were going to him. 5. This commandment I write to the brethren. 6. On that day he was preaching in the temple. 


\section{LESSON XXII}

\section{Present Subjunctive Active}

159.

VOCABULARY

$\dot{\alpha} \mu \alpha \rho \tau \dot{\alpha} \nu \omega, I \sin$

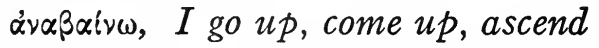

$\dot{\alpha} \nu \alpha$, prep. (original meaning on, upon, along) usually with the accusative in the distributive sense:

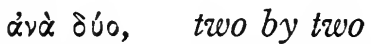

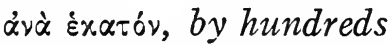

ivo, conj. generally with subjunctive, in order that, that

$x \alpha \tau \alpha$, prep. (original meaning down) with gen., down (upon), against; with abl., down (from); with acc., down (along), through, according to.

$\mu \varepsilon \tau \dot{\alpha}$, prep. (original meaning "midst") with the gen., with; with the acc., after; $\mu \varepsilon \tau \dot{\alpha} \tau \alpha \tilde{u} \tau \alpha$, after these things, after this.

$\mu \dot{n}, \quad$ not

vũy, adv., now $\chi \alpha i \rho \omega$, rejoice $\pi \tilde{\omega} \varsigma$, adv., how

160. From the previous lessons it has been learned that the Greek verb has tense, voice, and mode, like verbs in other languages.

161. It has been seen (I4) that tense has to do with the action of the verb as regards the state of action. Voice (5I and 52) has to do with the action of the verb as regards the subject of the action. Mode has to do with the manner of affirmation, how it is made, and not with action as do voice and tense. 
162. It has been observed that the indicative is the mode of definite assertion. It is used to affirm positively, definitely, absolutely, undoubtingly. The mode has nothing to do with the actual facts (whether true or untrue), but only with the statement of them. The indicative states a thing as true. 163. The subjunctive mode is a mode of doubtful statement, of hesitating affirmation, of contingency.

a. The subjunctive is usually found in two tenses, the present and the aorist. The perfect subjunctive is very rare.

164. The present subjunctive active of $\lambda \dot{\omega} \omega$ is:

\begin{tabular}{|c|c|}
\hline Singular & Plural \\
\hline I. $\lambda \dot{u} \omega$ & $\lambda \dot{u} \omega \mu \varepsilon \nu$ \\
\hline 2. úns & $\lambda u ́ \eta \tau \varepsilon$ \\
\hline 3. $\lambda$ ún & $\lambda \dot{u} \omega \sigma !\left(\nu^{1}\right)$ \\
\hline
\end{tabular}

165. Observe that: I. The subjunctive has the primary active personal endings (see I7-19). 2. The long thematic vowel $\omega / \eta$ is the subjunctive mode sign. 3. In the second and third persons sing. $\eta$ has iota-subscript ( $n$ )

166. The present subj. of $\varepsilon i \mu$ is:

\begin{tabular}{|c|c|}
\hline Singular & Plural \\
\hline I. $\dot{\omega}$ & $\tilde{\omega} \mu \varepsilon \nu$ \\
\hline 2. मूर & $\dot{\eta} \tau \varepsilon$ \\
\hline 3. $\hat{?}$ & $\tilde{\omega} \sigma l\left(\nu^{1}\right)$ \\
\hline
\end{tabular}

Note the circumflex accent.

167. The subjunctive is used in clauses of purpose after $\eta^{2} \alpha$. 
PRESENT SUBJ. MIDD. AND PASS.

Examine closely the following sentences:

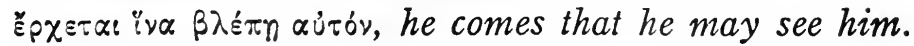
$\tau \alpha \tilde{\tau} \tau \alpha \lambda \dot{\varepsilon} \gamma \circ \mu \varepsilon \nu$ i $\nu \alpha \mu \dot{\gamma} \dot{\alpha} \mu \alpha \rho \tau \alpha \dot{\alpha} \nu \omega \sigma$, we say this in order that they may not sin.

168. Note that the negative with the subjunctive is $\mu$. 169. There is no time (absolute) element in the subjunctive mode in any tense. The present subjunctive expresses linear or durative action without reference to time. See I4.

170.

EXERCISES

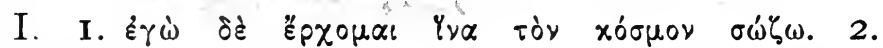

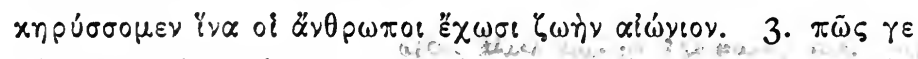

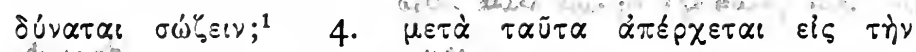

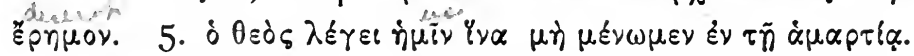

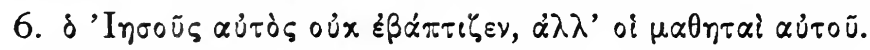

II. I. Now we become the children of God. 2. They baptize in order that they may glorify God. 3. You are not able to hear my word. 4. After these things he goes away in order that they may not see him. 5. How can (is able) he take away our sins?

\section{LESSON XXIII}

\section{Present Subjunctive Middle and Passive}

171. VOCABULARY

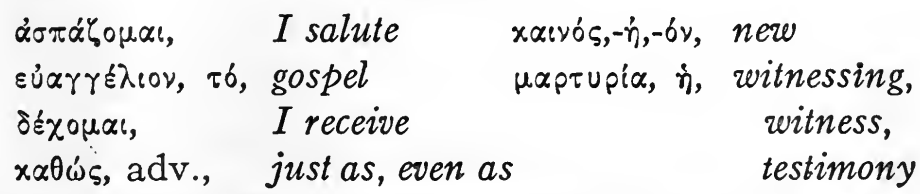

1 The question mark $(;)$ is the same in form as our semicolon. 


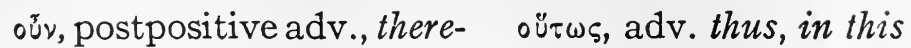
fore, then, now. manner, so

172. The present middle and passive subjunctive of $\lambda u ́ \omega$. is:
Singular
Plural
I. $\lambda \dot{u} \omega \mu \alpha$
$\lambda \cup \omega^{\prime} \mu \varepsilon \theta \alpha$
2. $\lambda$ ún
$\lambda u ́ n \sigma \theta \varepsilon$
3. $\lambda u ́ \eta \tau \alpha t$
$\lambda \dot{\omega} \omega \nu \tau \alpha \ell$

173. Observe: I. The middle and passive forms are alike (this was seen in the indicative also, 60).

2. The mode $\operatorname{sign} \omega / \eta$ is the same as in the active (see 165,2$)$.

3. The personal endings are the primary middle (and passive) endings (see 54 and 60 ).

$a$. In the second pers. sing. $-n$ is for $-\eta \sigma \alpha ! ; \sigma$ dropped out, then $\eta$ and $\alpha$ contracted to $n$. Note iota-subscript under $\eta$

174. The subjunctive (first person plural) is used in exhortations: as

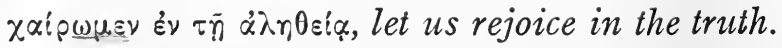

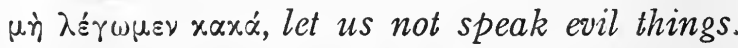

175. Many verbs in Greek are followed by the genitive case, and many by the dative case, where the corresponding verbs in English would be followed by the objective case. In each instance the idea of the case is accented.

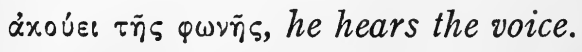

(This just tells "kind" of sound.) The accusative 
may be used after $\dot{\alpha}$ xoú $\omega$; then the meaning of the sound is comprehended.

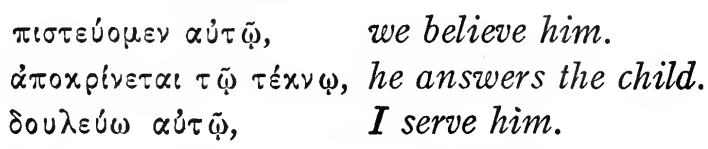

Note that the dative accents the personal interest.

176.

EXERCISES

I. I. $\pi\llcorner\sigma \tau \varepsilon \dot{\omega} \omega \mu \varepsilon \nu$ $\tau \tilde{\varphi}$ xupị́. 2. $\dot{\alpha} \gamma \dot{\omega} \mu \varepsilon \theta \alpha$ i் $\pi \dot{\delta} \quad \tau \tilde{\omega} \nu$

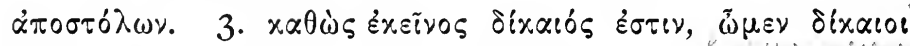

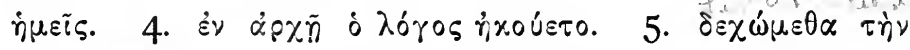

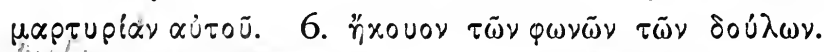

II. I. Let us not answer him. 2. Let us salute the apostles. 3. Let us receive the truth in order that we may know it. 4. He was preaching the gospel of the new covenant. 5. He is able to take away our sins. 6. Let us be led by the Lord into truth.

\section{LESSON XXIV}

\section{Second Aorist Indicative Active and Middle}

177. VOCABULARY

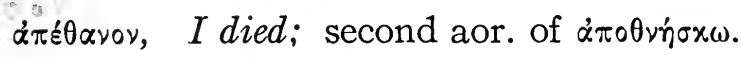

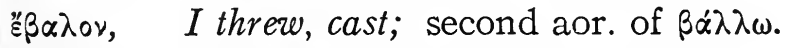

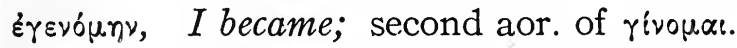

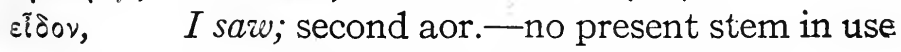
but $\delta \rho \alpha \dot{\omega} \omega$ is used in present tense.

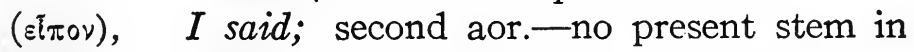
$\varepsilon i \pi \alpha, \quad$ use, but $\lambda \varepsilon \xi \omega$ is used in the present tense.

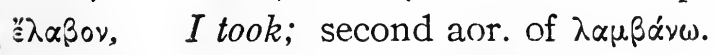


है $\lambda(\pi \circ v, \quad I$ left; second aor. of $\lambda \varepsilon i \pi \omega$.

Eijpov, I found; second aor. of supioxw.

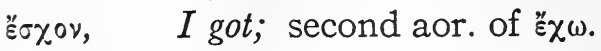

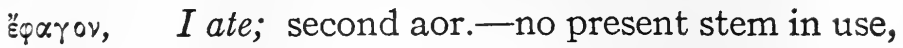
but $\varepsilon^{2} \sigma \theta i \omega$ is used in the present tense.

intov, I went, came; second, aor.-no present stem in use, but हैpxopal is used in the present tense.

$\pi \alpha \rho \hat{\varepsilon} \lambda \alpha \beta \beta \nu, I$ received; second aor. of $\pi \alpha \rho \alpha \lambda \alpha \mu \beta \alpha^{\prime} \nu \omega$.

178. The second aorist is so called in distinction from the first aorist, which is to be studied in Lesson XXXVI. They are not two different tenses, but second aorist and first aorist are two forms of the same tense.

179. As has already been learned (I4), the fundamental idea in tense is the "kind of action." The present tense (and imperfect, 65, 69) expresses durative or linear action. The aorist tense expresses action in its simplest form-undefined; it does not distinguish between complete or incomplete action. The aorist tense treats the action as a point;- this kind of action is called punctiliar: E $\chi \omega, I$ have, am holding; हَ̋xov, I got, obtained.

180. This kind of action (punctiliar) is timeless. But time is expressed in the indicative mode by the augment,- - punctiliar action in past time, generally.

In narrative the difference between the aorist indicative and the imperfect indicative is just this: the aorist indicative expresses punctiliar action in past time, while the imperfect indicative expresses durative action in past time. 
181. The second aorist ${ }^{1}$ indicative active and middle of $\lambda_{\varepsilon i} i \omega$ is:

\section{ACTIVE}

\begin{tabular}{|c|c|c|}
\hline Singular & & Plural \\
\hline I. $"$ है $\lambda: \pi \circ \nu$ & $21 \ldots 1$ & $\varepsilon \lambda_{i} i \pi \rho \mu \varepsilon \nu$ \\
\hline 2. हैं & & $\varepsilon \lambda i \pi \varepsilon \tau \varepsilon$ \\
\hline 3. "ै $\lambda_{i} \pi \varepsilon \varepsilon$ & & $\varepsilon^{\prime \prime} \lambda_{l} \pi \circ \nu$ \\
\hline
\end{tabular}

Second aorist active infinitive, $\lambda_{\iota} \pi \varepsilon i \nu$.

\section{MIDDLE}
I. $\varepsilon \lambda_{1} \pi \delta \mu \eta \nu$
$\varepsilon \lambda_{\imath} \pi \delta ́ \rho \varepsilon \theta \alpha$
2. $\varepsilon \lambda i \pi \circ u$
$\varepsilon \lambda i \pi \varepsilon \sigma \theta \varepsilon$
3. $\varepsilon \lambda i \pi \varepsilon \tau 0$
$\varepsilon \lambda i \pi 0 \nu \tau 0$

Second aorist middle infinitive, $\lambda_{\iota} \pi \varepsilon \sigma \theta \alpha \iota$.

182. Observe: I. The difference in form between the second aorist indicative and the imperfect indicative of the same verb is a difference in stem: aorist stem $\lambda_{\imath} \pi-;$ imperfect (having the present stem) $\lambda_{\varepsilon l \pi-.}$ 2. The secondary personal endings (66, 79) are used. 3. The augment in the aorist follows the same principles as it did in the imperfect. (70). 4. The accent of the second aorist infinitive is not recessive (II), but in the active is placed on the ultima, and in the midde on the penult.

The endings, $-\alpha,-\alpha \varsigma,-\varepsilon,-\alpha \mu \varepsilon \nu,-\alpha \tau \varepsilon,-\alpha \nu$, are found frequently with second aorist stems and almost exclusively with elimov.

183. Note that the infinitive has no augment. The aorist act. inf. $\lambda_{\iota} \pi \varepsilon i \nu$ means simply to leave, the action

${ }^{1}$ The second aorist of the thematic vowel $\%$ type is introduced here on account of its simplicity and its similarity in inflection to the imperfect, as well as on account of its frequent use. 
is punctiliar and timeless; while the present act. infinitive $\lambda \varepsilon i \pi \varepsilon \iota \nu$ means to be leaving (or to keep leaving), the action is durative and timeless. So also in the middle.

184. The second aorist usually exhibits the simple stem of the verb. The second aorist given here is the thematic type, i.e., uses the thematic vowel $\%$.

\begin{tabular}{|c|c|c|c|c|}
\hline & $\begin{array}{c}\text { Present } \\
\text { stem }\end{array}$ & $\begin{array}{c}\text { Present } \\
\text { theme }\end{array}$ & $\begin{array}{l}\text { Aorist } \\
\text { stem }\end{array}$ & $\begin{array}{l}\text { Aorist } \\
\text { theme }\end{array}$ \\
\hline$\beta \alpha^{\prime} \lambda \lambda \omega$ & $\beta \alpha \lambda \lambda-$ & $\beta \alpha \lambda \lambda \circ / \epsilon$ & $\beta \alpha \lambda-$ & $\beta \alpha \lambda \circ / \epsilon$ \\
\hline Yivouat & $\gamma(\nu-$ & $\gamma(\nu) / \epsilon$ & YEv- & $\gamma \varepsilon \nu^{\circ} / \epsilon$ \\
\hline$\lambda \varepsilon i \pi \omega$ & $\lambda \varepsilon 1 \pi-$ & $\lambda \varepsilon i \pi^{\circ} / \epsilon$ & $\lambda_{6 \pi-}$ & $\lambda_{l} \pi^{0}$ \\
\hline
\end{tabular}

Note that the second aorist is known by its stem.

185. From the forms of the present, imperfect, perfect, etc., it cannot be determined beforehand whether a verb has a first aorist or a second aorist, nor, if it has a second aorist, what the form of the second aorist is. To determine this, the verb must be examined in a lexicon.

186. The second aorist act. and middle are formed on the second aorist stem. The aorist passive of all verbs is different from the aorist middle. Review the meaning of the middle (52).

$\varepsilon \varepsilon^{2} \iota \pi \sigma_{\mu \eta \nu}$ is second aorist indicative middle, I left for myself, etc.

187. The Greek aorist indicative is not the exact equivalent of any tense in English or in any other language. The Greek aorist and the English preterit do not exactly correspond. The translation given in the vocabulary is just to get the verb idea asso- 


\section{SECOND AORIST SUBJUNCTIVE}

ciated with the verb form. To translate the Greek aorist ind., sometimes the English preterit is used, sometimes the perfect, sometimes the past. The Greek aorist ind. refers the action to the past without any exact specification as to antecedence of action or as to present results of action.

188.

EXERCISES

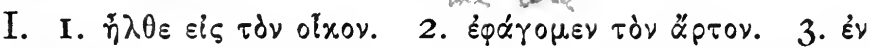

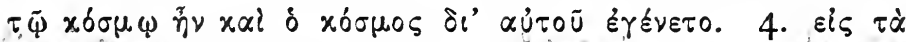

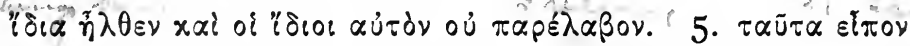

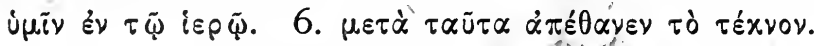

II. I. He died on the third day. 2. He took the bread and ate (it). 3. The disciples obtained good promises. 4. They came and saw where $(\pi \circ \tilde{u})$ he was abiding. 5. The servant cast a stone into the boat.

\section{LESSON XXV}

Second Aorist Subjunctive Active and Middle

189.

VOCABULARY

$$
\begin{aligned}
& \dot{\alpha} \lambda \eta \eta \bullet v o ́ s,-\dot{\eta},-\delta v, \text { true } \\
& \text { Bios, } \delta, \quad \text { life Exxei, adv., there }
\end{aligned}
$$

Learn the capital letters in I of Lesson I.

190. The second aorist subjunctive active and middle of $\lambda \varepsilon^{i} \pi \omega$ is:

Singular

I. $\lambda i \pi \omega$

2. $\lambda i \pi n s$

3. $\lambda i \pi n$
Active

\section{Plural}

$\lambda i \pi \omega \mu \varepsilon \nu$

$\lambda i \pi \eta \tau \varepsilon$

$\lambda$ i $\pi \omega \sigma$ 


\section{MiddLe}
I. $\lambda\{\pi \omega \mu \alpha$ t
$\lambda เ \pi \omega \mu \varepsilon \theta \alpha$
2. $\lambda i \pi n$
3. $\lambda i \pi \eta \tau \alpha !$
$\lambda i \pi \eta, \theta \varepsilon$
$\lambda(\pi \omega \nu \tau \alpha)$

191. Observe: I. There is no augment in the aorist subjunctive. 2. The personal endings are the primary active and middle. 3. The subjunctive mode sign is the long thematic vowel $\omega / \eta$. 4. The only difference in form between the second aorist subj. (act. and middle) and the present subj. (act. and middle) is in the stem, - present stem $\lambda_{\varepsilon} \iota \pi-$; aorist stem $\lambda \iota \pi$.

192. Let it be remembered that the aorist subjunctive does not denote past time. But the real time of the subj. is future in relation to the speaker or writer; and this time element is not due to the tense at all.

193. The distinction in meaning between the present subjunctive and the aorist subjunctive is only in the kind of action. The present subj. expresses durative action. The aorist subj. expresses punctiliar action.

Example: $\mu \dot{\gamma} \dot{\alpha} \pi \dot{\delta} \theta \dot{\gamma} \sigma \alpha \omega \mu \varepsilon \nu \varepsilon \dot{\varepsilon} \tau \tilde{\eta} \dot{\alpha} \mu \alpha \rho \tau\{\alpha$, let $u s$ not be dying in sin, let us not continue to die (or keep on dying) in sin. $\mu \hat{\gamma} \dot{\alpha} \pi 0 \theta \dot{\alpha} \nu \omega \mu \varepsilon \nu$ हैy $\tau \tilde{n} \dot{\alpha} \mu \alpha \rho \tau i \alpha$, let us not die in sin.

In the first example the present (subjunctive) represents the action in progress. In the second example the aorist (subjunctive) just treats the action as a single whole without any reference to 


\section{THIRD DECLENSION}

progress or completion. It is generally difficult to bring out the difference in an English translation.

194. The subjunctive is used in questions of doubt, where the speaker asks what he is to do or say:

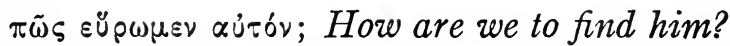
$\tau i$ हli $\pi \omega$; What am I to say? What shall I say?

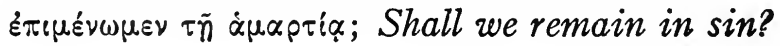

195.

EXERCISES

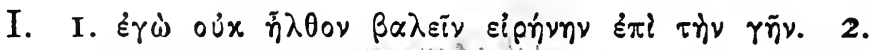

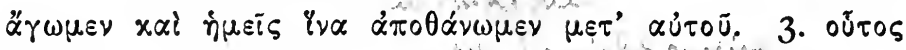

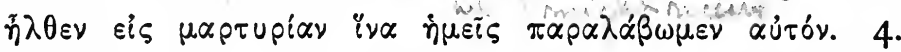

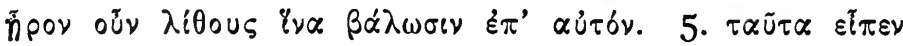

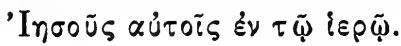

II. I. How shall we receive them? 2. Let us not become evil prophets. 3. What shall we eat? 4. The servants came in order that they might find the children. 5. He died that men might have life.

\section{LESSON XXVI}

Third Declension: Neuter Substantives in - $\mu \alpha \tau-$. 196.

VOCABULARY

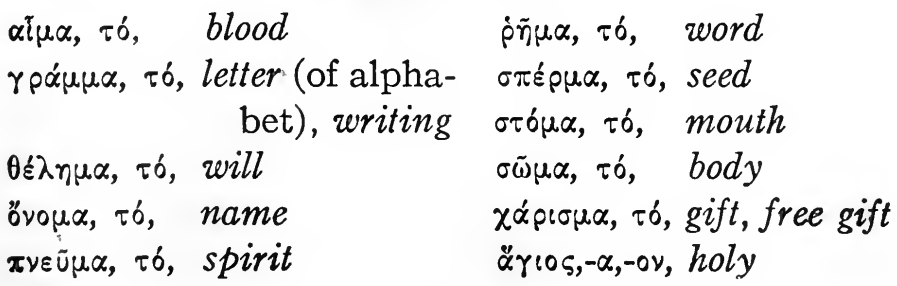


197. The third declension is commonly called the consonant declension because most of the nouns have stems ending in a consonant. A few nouns, included in this declension, ended in the vowels : and $u$, which were sometimes semivowels.

198. The case endings of the consonant declension are:

Singular

Plural

Masc. and Fem. Neut. Masc. and Fem. Neut.

Nom. $-\varsigma$ or none None

Gen. -05

$-05$

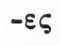

$-\alpha$

Abl. -05

$-05$

Loc. - -

$-8$

Ins. - -

Dat. - -

Acc. $-\nu$ or $-\alpha$

$-6$

$-8$

Voc. none or like none nom. or stem

In the case endings final $\alpha$ is short.

These forms must be thoroughly mastered.

Note that the loc., ins., and dat. sing. use the locative ending $-t$; and in the plural these cases use the locative ending -ot (see p. 65 n.).

The gen. and abl. plural ending $-\omega \nu$ is the same for all the declensions.

The neuter plural nom., acc., and voc. are always alike.

The vocative plural is always the same as the nominative. 
199. The declension of ${ }^{2} \nu \circ \mu \alpha,{ }^{1}$ name, is:

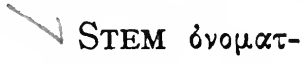

Singular

Nom. övoux

Gen. $\delta$ ó $\mu \alpha \tau \circ s$

Abl. ovó $\mu \alpha \tau o s$

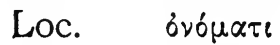

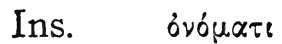

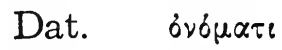

Acc. övore
Plural

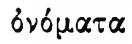

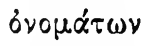

ò $о \mu \alpha \dot{\tau} \tau \omega$

ovós $\mu \sigma \sigma$

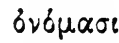

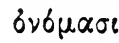

б $\delta \mu \alpha \tau \alpha$

200. Observe: I. As in the neuters of the second declension, the nom. acc. and voc. sing. are alike, and the same cases in the plural are alike. 2. The nom. sing. is the mere stem, final $\tau$ being dropped.

Note: The consonants that can stand at the close of a word in Greek are $\nu, \rho$, and $\varsigma$ (including $\xi$ and $\psi$ ). All other consonants which would occur there are

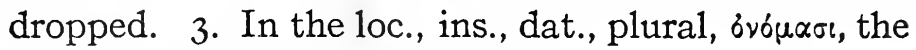
$\tau$ of the stem drops out before $\sigma$.

201. In declining a substantive of the consonant leclension it is necessary to know the stem. The stem is usually found by dropping the genitive sing. ending -os. Thus: Nom. $\sigma \tau \delta \mu \alpha$; gen. $\sigma \tau \delta \mu \alpha \tau o s ;$ stem

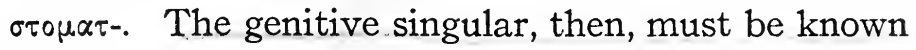
before nouns of the consonant declension can be declined.

202. The gender of the third declension substantives, except in the case of special classes like the sub-

1 Neuter substantives with nom. in $-\mu \alpha$ (stem $-\mu \alpha \tau-)$ are introduced first on account of their simplicity and importance. 
stantives in $-\mu \alpha \tau-$, must be learned with each word separately.

203. A neuter plural subject often has its verb in the singular:

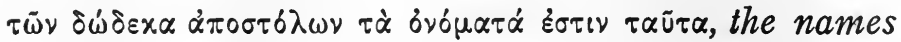
of the twelve apostles are these.

204.

EXERCISES

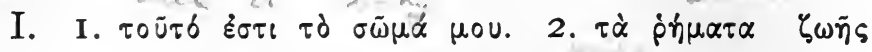

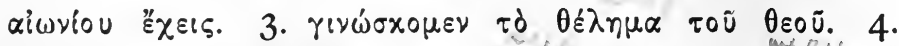

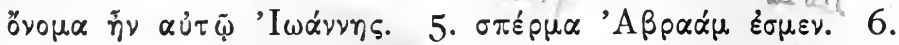

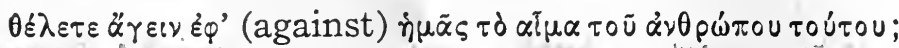

II. I. This is the will of God. 2. These are the good seed. 3. The words of the prophet are written in the scriptures. 4. Let us believe on (zis) the name of the Lord. 5. That one was baptizing them in the holy spirit. 6. The will of God came through the mouths of the prophets.

\section{LESSON XXVII}

\section{Future Indicative Active and Middle}

205.

VOCABULARY

$\delta \alpha \iota \mu \dot{v} v\llcorner o \nu, \tau o ́$, demon

$x \alpha$, besides the usual connective use, $a n d$, is used in the sense of also, and even.

$\pi \varepsilon p^{\prime}$, prep. (original meaning around (on all sides)) with gen., about, concerning; with abl., from around; with acc., round about, about, concerning. ixép, prep. (original meaning over, upper) with abl., in behalf of, in interest of; instead of; in place of: 
for the sake of; about, concerning; with acc., over, above, beyond.

$\dot{\omega} s$, relative, comparative, and temporal adv., as, when.

206. The future tense is made on aoristic (punctiliar) roots in some verbs and on durative roots in other verbs. The kind of action of the future may be either punctiliar or durative. But in use the future is generally punctiliar.

207. The future indicative, as in English, generally denotes what is going to take place. It is just the present vividly projected into the future. In English it is done by "shall" in the first person and by "will" in the second and third persons. Yet the future ind. has modal aspects which will be presented later.

208. The future indicative active and middle of $\lambda \dot{u} \omega$ is:

\section{ACtive}

Singular

I. 入úow, I shall loose

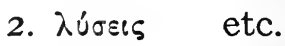

3. $\lambda u ́ \sigma \varepsilon \iota$
Plural

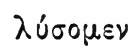

$\lambda \dot{\sigma} \sigma \varepsilon \tau \varepsilon$

$\lambda$ úgouge

Future active infinitive. $\lambda \dot{\sigma} \sigma \varepsilon \iota^{1}$

Middle

Singular Plural
I. $\lambda \dot{\sigma} \sigma o \mu \alpha \iota$,
I shall loose myself
$\lambda \cup \sigma o ́ p \varepsilon \theta \alpha$
2. $\lambda \dot{u} \sigma n$ or for myself, etc.
3. $\lambda \dot{\sigma \varepsilon \varepsilon \tau \alpha \ell ~}$
$\lambda \dot{u} \sigma \varepsilon \sigma \theta \varepsilon$

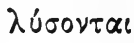

Future middle infinitive, $\lambda \dot{\sigma} \sigma \varepsilon \sigma \theta \alpha t$

1 The future infinitive is found only six times in the New Testa-

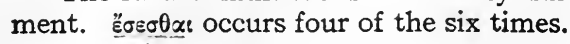


a. The future passive is quite different from the future middle. $b$. Except in the future and aorist, the middle and passive are alike in form.

209. Observe: I. The primary active and middle personal endings are used. 2. The tense-suffix is $\sigma$, added to the root $\lambda u-.3$. The future stem is $\lambda u \sigma-$. 4. The thematic vowel is $\%$. Note that, while the present theme is $\lambda 0^{\circ} / \epsilon$, the future theme is $\lambda 0^{\circ} / \epsilon$.

210. Most verbs whose verb stem ends in a single vowel (except the vowels $\alpha, \varepsilon$, and 0 ) or a diphthong are conjugated in the future like $\lambda \dot{v} \sigma \omega, \lambda \dot{\sigma} \sigma o \mu \alpha$.

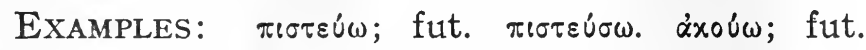

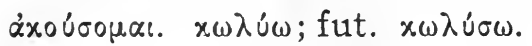

211. Future indicative of $\varepsilon i \mu i$ is:

Singular

I. हैंous:,

2. है०ก

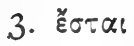

Plural

ह̇øó $\mu \varepsilon \theta \alpha$

$\ddot{\varepsilon} \sigma \varepsilon \sigma \theta \varepsilon$

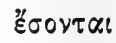

Future infinitive, है $\sigma \varepsilon \sigma \theta \alpha$.

212. Conditional sentences (see I49). The third class conditional sentence is the condition undetermined, but with prospect of determination. Here the subjunctive after $\varepsilon^{\prime} \alpha$ (if) is used in the condition (if) clause. The conclusion naturally has the future indicative, but may have any tense of the indicative, subjunctive, or imperative.

EXAMPLES:

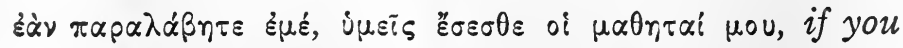
will receive me, you shall be my disciples. 


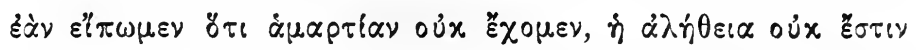
Eे iniv, if we say that we have not sin, the truth is not in us.

213.

\section{EXERCISES}

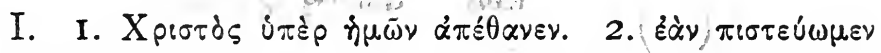

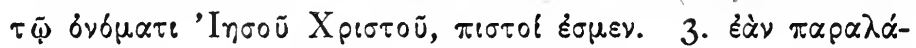

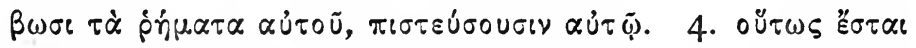

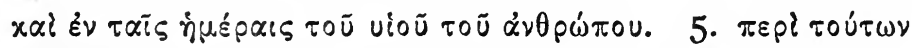

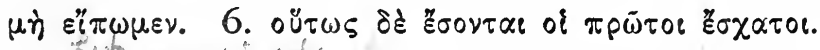

II. I. The sons of men shall believe on (zis) the word of God. 2. There shall be joy in heaven because $(" \tau \tau)$ he is saved. 3. Ye shall be with ( $\mu \varepsilon \tau \dot{\alpha})$ me this day. 4. If he believe me, I shall hear him. 5. Let us go to him.

\section{LESSON XXVII}

Future Indicative Active and Middle (Continued)

214.

VOCABULARY

\begin{tabular}{|c|c|}
\hline$\dot{\alpha} \gamma \alpha \pi \dot{\alpha} \omega$, & I love \\
\hline 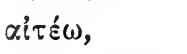 & I ask for (something) \\
\hline$\alpha \dot{x} 0 \lambda \circ \cup \theta \varepsilon \hat{\varepsilon} \omega$ & I follow \\
\hline 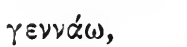 & $I$ beget \\
\hline$\varepsilon \dot{\rho} \omega \tau \tau \dot{\alpha} \omega$. & I ask (question) \\
\hline$\zeta \dot{\alpha} \omega$, & I ive \\
\hline$\zeta \eta \tau \varepsilon \omega$, & I seek \\
\hline$\lambda \alpha \lambda \hat{\varepsilon} \omega$, & I speak \\
\hline$\mu \alpha \rho \tau \cup \rho \varepsilon \omega$, & I bear witness, testify \\
\hline$\pi \alpha \rho \alpha x \alpha \lambda \varepsilon \omega$ & I beseech, exhort, encourage \\
\hline
\end{tabular}




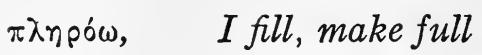

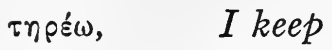

$$
\begin{aligned}
& \text { qavepów, make manifest }
\end{aligned}
$$

Notice that the present stem of these verbs ends in one of three vowels, $\alpha, \varepsilon$, or 0 . These are called contract verbs because in the conjugation of the present system these vowels contract with the thematic vowel (and personal ending). These verbs in $-\dot{\alpha} \omega,-\varepsilon \omega$, and - $\omega \omega$ are contracted only in the present and imperfect, and will be studied in Lessons XLIX, etc. In all other systems these verbs, if regular, are conjugated like the corresponding tenses of $\lambda \hat{\omega} \omega$.

215. The future of

$$
\begin{array}{ll}
\dot{\alpha} \gamma \alpha \pi \dot{\alpha} \omega & \text { is } \dot{\alpha} \gamma \alpha \pi \dot{\eta} \sigma \omega ; \\
\lambda \alpha \lambda \varepsilon \omega & \text { is } \lambda \alpha \lambda \hat{\eta} \sigma \omega ; \\
\varphi \alpha \nu \varepsilon \rho \delta \omega & \text { is } \varphi \alpha \nu \varepsilon p \hat{\omega} \sigma \omega .
\end{array}
$$

From these forms it is evident that verbs whose stems end in a short vowel $(\alpha, \varepsilon, 0)$ generally lengthen that vowel before $-\sigma^{0} / \epsilon^{1}$ of the future, and then are conjugated like $\lambda \dot{\sigma} \sigma \omega$ (see 208).

$\alpha$ is lengthened to $\eta$ (but $\alpha$ after $\varepsilon$, l, or $\rho$ is length$\varepsilon$ is lengthened to $\eta \quad$ ened to $\alpha$ not $\eta$ ).

0 is lengthened to $\omega$ Thus:

$$
\begin{aligned}
& -\alpha+\sigma^{\circ} / \epsilon=-\eta \sigma^{\circ} / \epsilon \\
& -\varepsilon+\sigma^{\circ} / \epsilon=-\eta \sigma^{\circ} / \epsilon \\
& -0+\sigma^{\circ} / \epsilon=-\omega \sigma^{\circ} / \epsilon
\end{aligned}
$$

216. I. The future of

$$
\begin{aligned}
& \beta \lambda \varepsilon \dot{\varepsilon} \omega \text { (stem } \beta \lambda \varepsilon \pi-) \text { is } \beta \lambda \varepsilon \varepsilon \psi \omega \text {; }
\end{aligned}
$$

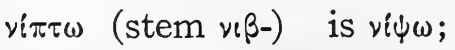

$$
\begin{aligned}
& \gamma \rho \alpha \dot{\varphi} \varphi \omega \text { (stem } \gamma \rho \alpha \varphi-\text { ) is } \gamma \rho \alpha \dot{\psi} \psi \omega \text {. }
\end{aligned}
$$

1 This is also true of other tense-suffixes. 
From this it is seen that stems in $\pi, \beta$, or $\phi$ unite with $\sigma$, forming $\psi$; that is $\pi+\sigma=\psi ; \beta+\sigma=\psi$; $\varphi+\sigma=\psi$.

2. The future of

$$
\begin{aligned}
& \delta \imath \dot{\omega} x \omega \text { (stem } \delta \iota \omega x-\text { ) is } \delta \iota \omega^{\prime} \xi \omega \text {; } \\
& \ddot{\alpha} \gamma \omega \quad \text { (stem } \dot{\alpha} \gamma-) \text { is } \ddot{\alpha} \xi \omega \text {; } \\
& \varepsilon \chi \omega \text { (stem } \sigma \varepsilon \chi-) \text { is } \varepsilon \xi \omega \text {. }
\end{aligned}
$$

Thus it is seen that stems in $x, \gamma$, or $\chi$ unite with $\sigma$ forming $\xi$; that is, $x+\sigma=\xi ; \gamma+\sigma=\xi ; \chi+\sigma=\xi$.

3. The future of

$$
\begin{aligned}
& \left.\sigma \omega \zeta_{\omega} \text { (stem } \sigma \omega \delta-\right) \text { is } \sigma \omega \sigma \omega \text {; } \\
& \pi \varepsilon i \theta \omega \text { (stem } \pi \varepsilon \imath \theta_{-} \text {) is } \pi \varepsilon i \sigma \omega .
\end{aligned}
$$

From this it is seen that stems in $\tau, \delta$, or $\theta$ drop $\tau$, $\delta$, or $\theta$ before $\sigma$, leaving simple $\sigma$; that is, $\tau \sigma=\sigma$; $\delta \sigma=\sigma ; \theta \sigma=\sigma$.

217. Certain consonants are called mutes or stops because in forming them the passage of the breath is for a moment closed.

$\checkmark$ I. The consonants $\pi, \beta, \phi$ are called labial mutes or stops, because they are made with the lips.

2. $x, \gamma, \chi$ are called palatal mutes or stops, because they are made with the soft palate.

3. $\tau, \delta, \theta$ are called lingual (or dental), because they are made with the tongue (or teeth).

218. As an aid to memory the changes in mutestems in forming the future may be exhibited thus:

Labials, $\pi, \beta, \varphi+\sigma=\psi$.

Palatals, $x, \gamma, \chi+\sigma=\xi$.

Linguals, $\tau, \delta, \theta+\sigma=\sigma$. 
Keep in memory this order of the mutes. They occasion many important changes in formation of words.

219. The verb-stem in many verbs is not the same as the present stem: in the case of $\nu i \pi \tau \omega$ it was observed that the verb-stem is $v \bullet \beta-;$ the present stem $\nu \pi \pi \tau-;$ and the verb-stem of $\sigma \omega \dot{\zeta} \omega$ is $\sigma \omega \delta .^{1}$ The verb-stem of $\varphi v \lambda \alpha \sigma \sigma \omega$ is $\varphi \cup \lambda \alpha \alpha-;$ the future is $\varphi v \lambda \alpha \xi \omega$. The future of $\left.\beta \alpha \pi \tau i \zeta_{0} \omega\right)$ ( $\left.\beta \alpha \pi \tau i \delta-\right)$ is $\beta \alpha \pi \tau i \sigma \omega$.

220. The future of any word cannot be certainly determined beforehand. A verb may make its future on a durative root, a punctiliar (aoristic)

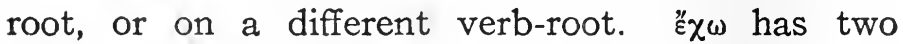
futures, $\check{\varepsilon} \xi \omega^{2}$ (durative) and $\sigma \chi \hat{\eta} \sigma \omega$ (punctiliar).

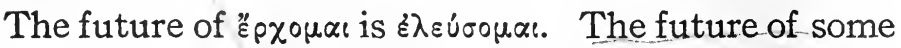
verbs occurs only in the middle voice: e.g., $\gamma(v \omega \sigma \times \omega$, future $\gamma \nu \omega \dot{\sigma o \mu \alpha}$. In general a lexicon must be consulted for each verb.

The future of verbs with liquid stems $(\lambda, \mu, \nu, \rho)$ will be given in Lesson XLVI.

221.

\section{Exercises}

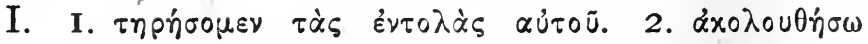

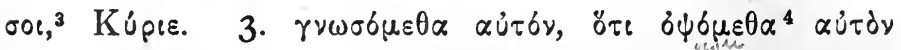

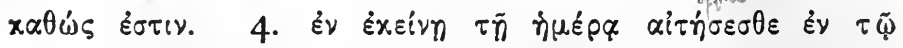

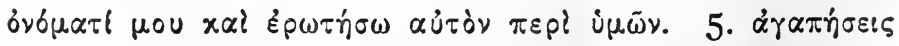

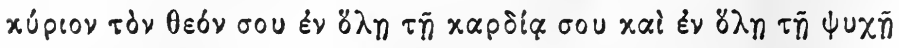

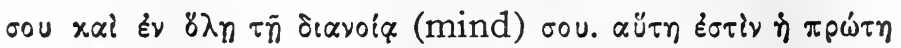

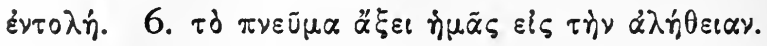

$1 \zeta$ is treated as a combination of $\delta+z$ (or $z \delta$ ).

${ }^{2}$ Note the rough breathing on $\tilde{\xi} \xi \omega$.

${ }^{3}$ axo $\lambda_{0} 0 u \theta \omega$ is followed by the associative-instrumental case.

4 From ö $\pi \tau 0 \mu \alpha$, I see; stem óx-. 
II. I. We shall know the truth and do it. 2. We shall glorify God. 3. They will bear witness concerning him. 4. We shall persuade our hearts. 5. He will baptize you. 6. The faithful will proclaim the word of God.' 7. I shall do the will of God.

\section{LESSON XXIX}

\section{Third Declension: Lingual Mute Stems}

222.

VOCABULARY

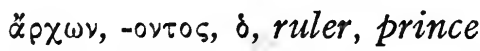

$$
\begin{aligned}
& \varepsilon \lambda \pi i \varsigma,-i \delta \circ \varsigma, \dot{\eta} \text {, hope }
\end{aligned}
$$

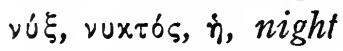

$$
\begin{aligned}
& \pi \circ u \dot{s}, \pi 0 \delta \sigma \varsigma, \delta, \text { foot }
\end{aligned}
$$

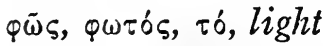

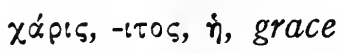

$$
\begin{aligned}
& \text { Eprasia, } \eta \text {, work, business }
\end{aligned}
$$

223. Note that the stem of

$$
\begin{aligned}
& \varepsilon \lambda \pi i \zeta \text { is } \varepsilon \lambda \pi \imath \delta \text {-; } \\
& \text { xäpis is xapir-; }
\end{aligned}
$$

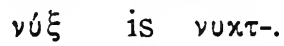

Observe that the stem in all these substantives end in a lingual mute, and that the nominative is formed by adding $s$ to the stem. Thus,

$$
\begin{array}{lll}
\varepsilon \lambda \pi \imath \delta \varsigma & \text { becomes } & \varepsilon \lambda \pi i \varsigma ; \\
\chi \alpha \rho \iota \tau \varsigma & \text { becomes } & \chi \alpha \dot{\alpha} \iota \varsigma ; \\
\nu \cup x \tau \varsigma & \text { becomes } & \nu \cup x \varsigma=\nu \cup \xi
\end{array}
$$

For the changes that occur when a mute and 5 come together see 216 and 218 . 


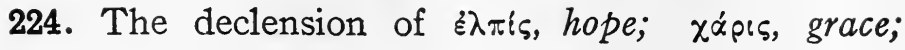
and vú $\xi$, night:

STEM $\varepsilon \lambda \pi \iota \delta-$

STEM $\chi \alpha \rho i \tau-$

Singular

Nom.

* Gen.

Abl.

Loc.

Ins.

Dat.

Acc.

Nom.

Gen.

Abl.

Loc.

Ins.

Dat.

Acc.

$\varepsilon \lambda \pi i \varsigma$
$\varepsilon \lambda \pi i \delta \circ \varsigma$
$\varepsilon \lambda \pi i \delta \circ \varsigma$
$\varepsilon \lambda \pi i \delta l$
$\varepsilon \lambda \pi i \delta l$
$\varepsilon \lambda \pi i \delta l$
$\varepsilon \lambda \pi i \delta \alpha$

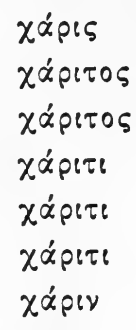

Plural

$a$. In the accusative singular forms like $\varepsilon \lambda \pi i \delta \alpha \nu$, vuxiav, are found. $b$. In the acc. singular, the form $\chi \alpha \dot{\rho}, \tau \alpha$ often occurs. $c$. Vocatives in this declension are rare and will be specially mentioned whenever separate forms occur.

225. Observe that: I. In the loc. ins. and dat. plu. a lingual mute drops out before - $\sigma$ (see $216,3,218$ ).

2. In the case of $v \cup \xi i, x \sigma$ changes to $\xi$ after $\tau$ dropped out.

226. Monosyllables of the third declension generally have the accent on the ultima in the gen., abl., 
loc., ins., and dat. of both numbers. In the gen. plu. $\tilde{\omega} \nu$ has the circumflex. But $\varphi \tilde{\omega} \varsigma$ and $\pi \alpha \tilde{i} \varsigma$ are accented in gen. plu. thus, $\varphi \omega \dot{\tau} \tau \omega v, \pi \alpha i \delta \omega \nu$.

227. When substantives with stems in $-\imath \tau,-\imath \delta$, or $-\imath \theta$ are not accented on the ultima, the acc. sing. generally has $\nu$ in place of the mute $(\tau, \delta, \theta)$, e.g., $\chi \alpha$ pess ( $\chi \alpha$ per-), acc. $\chi \alpha \dot{\alpha} p \iota \nu$ (but see $224 b$ ); but when the accent is on the ultima, the acc. is generally formed like the acc. of $\dot{\varepsilon} \lambda \pi i \varsigma, \dot{\varepsilon} \lambda \pi i \delta \alpha$.

228. Declension of $\ddot{\alpha}_{p} \chi \omega \nu$, ruler, is:

$\checkmark$ STEM ápxovt-

\section{Singular}

Nom. $\quad \ddot{\rho} \rho \omega \nu$

Gen. «ँpxovios

Ab1. $\ddot{\alpha} \rho x \circ \nu \tau 0 s$

Loc. $\quad \ddot{\alpha} \rho \chi 0 \nu \tau$

Ins. $\quad \ddot{\rho} \rho x \circ \nu \tau$

Dat. $\ddot{\alpha} \rho x \circ \nu \tau$

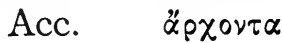

Plural

$\ddot{\alpha} \rho \chi \circ \nu \tau \varepsilon \varsigma$

$\dot{\alpha} \rho \chi \delta ́ v \tau \omega \nu$

$\dot{\alpha} \rho x \delta \nu \tau \omega \nu$

äpxovos

äpxovor

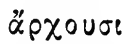

$\ddot{\alpha} \rho \chi \circ \nu \tau \alpha \varsigma$

229. Observe that: I. The nom. sing. of $\ddot{\alpha} \rho \chi \omega \nu$ is formed from the mere stem without adding anything; final $\tau$ of the stem is dropped, for a Greek word cannot end in $\tau$ (see 200, 2 note); then 0 of the stem is lengthened (formative lengthening) to $\omega$. 2. When $-\nu \tau$ - of the stem comes before $-\sigma$ of the loc., ins., and dat. plur., both $\nu$ and $\tau$ are dropped and the $\circ$ of the stem is lengthened to ou (compen-

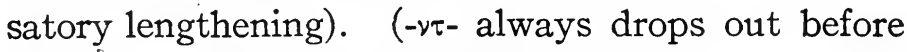
$-\sigma$ and the preceding vowel lengthened.) 
230. In expressions of time the locative denotes a point in which; the accusative duration of, and the genitive time within which (kind of time): e.g., vuxti

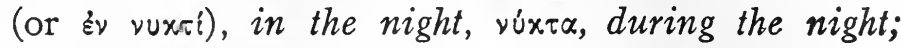
vuxiós, at night (not day).

231.

\section{EXERCISES}

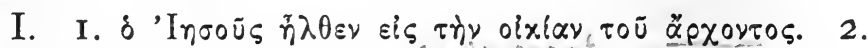

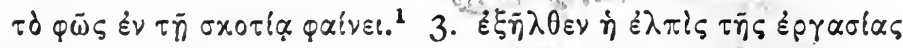

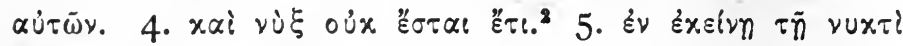

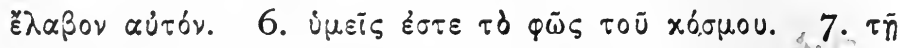

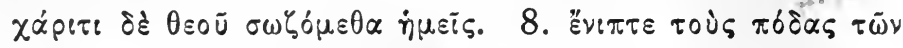

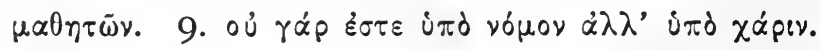

II. I. They shall bear witness concerning him that $(" \ddot{\imath} \tau)$ he is the light. 2. We are saved by grace. 3. We are not under law but under grace. 4. We have hope in God. 5. The ruler came to him at night.

\section{LESSON XXX}

Participles: The Present, Active, Middle, and Passive 232. VOCABULARY

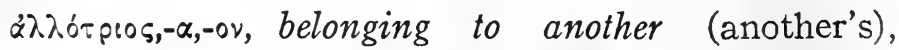
strange

äprl, adv., now, just now, this moment

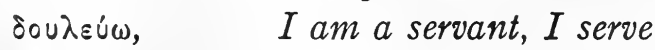

in, conj., or

xарло́s, $\delta, \quad$ fruit

$\tau \cup థ \lambda \hat{\sigma} \varsigma,-\dot{\eta},-6 v$, blind

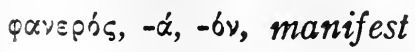

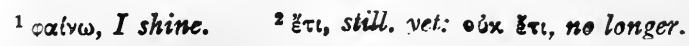




\section{PRESENT PARTICIPLE}

233. The present active participle of $\lambda \dot{\omega} \omega$ :

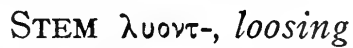

Masc. Singular

Nom $\lambda \dot{u} \omega y$

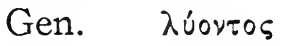

$\lambda$ úovod

Neut.

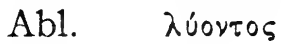

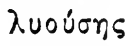

$\lambda$ บ̃ov

Loc. úovte

גuoúns

$\lambda$ Úoveos

Ins. $\lambda$ úoyte

Dat. $\lambda$ úoves

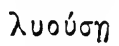

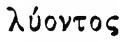

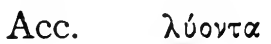

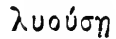

$\lambda$ úovet

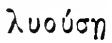

$\lambda$ Úover

$\lambda$ Úovolv

$\lambda$ úovet

$\lambda \tilde{\text { บ̃o }}$

\section{Plural}

\begin{tabular}{|c|c|c|c|}
\hline Nom. & 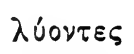 & $\lambda$ Úovoal & $\lambda$ úovia \\
\hline Gen. & $\lambda u \delta v \tau \omega \omega \nu$ & $\lambda \cup \circ \cup \sigma \tilde{\omega} \nu$ & 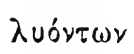 \\
\hline $\mathrm{Abl}$. & 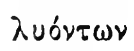 & $\lambda \cup \circ \cup \sigma \tilde{\omega} \nu$ & 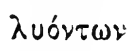 \\
\hline Loc. & $\lambda$ Úover & 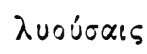 & $\lambda$ Úover \\
\hline Ins. & $\lambda$ Úovor & $\lambda$ uoúoهis & $\lambda$ úovat \\
\hline Dat. & $\lambda$ Úovor & 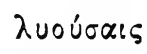 & $\lambda$ Úovar \\
\hline Acc. & $\lambda$ Úovras & $\lambda$ uoú $\sigma \alpha \varsigma$ & 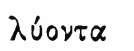 \\
\hline
\end{tabular}

234. Observe: I. The participle is declined in three genders.

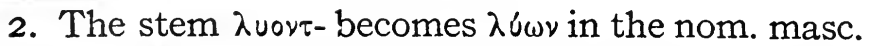
sing., like $\ddot{\alpha} p \omega \nu$, and is declined like $\ddot{p} \chi \omega \nu$.

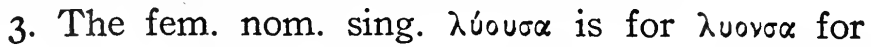

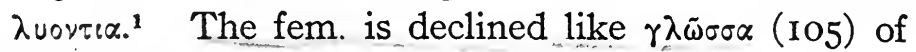
the first declension.

4. The neuter nom. and acc. sing. uov is the simple stem, final $\tau$ being dropped. $(229,200,2$. $)$

${ }^{1} \iota \alpha$ is the feminine suffix added to the stem. Apparently $\tau$ ( $\iota$ is a semivowel) became $\sigma$, then $\nu$ was dropped before $\sigma$ and $\sigma$ lengthened (compensatory) to ou. 
The neuter nom. and acc. plur. ends in $-\alpha$. Otherwise the neuter forms are like the masculine.

5. The masculine and neut. are declined in the third declension; the fem. in the first.

6. Note that participles are accented like adjectives; but the gen. and abl. fem. plural have the circumflex accent over the ultima like substantives of the first declension The accent is not recessive. The accent of the present active participle of $\dot{\alpha} \times 0 \dot{\omega} \omega$ is $\dot{\alpha} \times 0 \dot{v} \omega \nu, \dot{\alpha} \times \dot{v}^{\prime} \circ v \sigma \alpha, \dot{\alpha} \times 0 \tilde{u} \circ \nu$. Observe the position of the accent in the neuter-not recessive.

235. Learn the pres. participle of $\operatorname{si\mu i}(\S \mathrm{I} 6)$.

236. The future active participle of $\lambda u ́ \omega$, viz., $\lambda u ́ \sigma \omega \nu$,

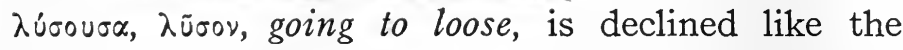
present participle of $\lambda \dot{u} \omega$. It is rare in the New Testament.

237. The present middle and passive participle of $\lambda u ́ \omega$ is:

\section{MIdDLE}

Masc. Fem. Neuter

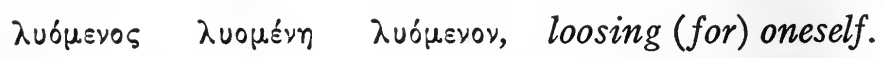

PAssive

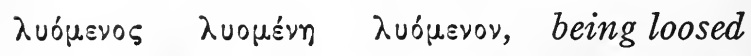

Observe that the present middle and passive participles are alike in form.

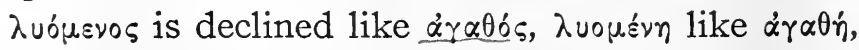
and $\lambda$ uórevov like $\alpha \gamma \alpha \theta \dot{\delta}$, except in accent. Thus it is seen that the present, middle, and passive participles are declined like adjectives of the first and second declensions. 
238. The future middle participle of $\lambda \dot{v} \omega$ is $\lambda \cup \sigma \delta \mu \varepsilon v \circ \varsigma$, $-\eta$, -ov, going to loose (for) oneself; and is declined like $\lambda$ uó $\mu \varepsilon v \circ \varsigma,-\eta,-o \nu$. This is also rare in the New Testament.

239. It is to be observed that the present participles are made on the present stem, and the future act. and middle participles are made on the future stem.

In a mechanical way, the present active, middle, and passive participles of any regular verb may be formed by adding $-\omega \nu,-o v \sigma \alpha,-o \nu$ and $-\mu \varepsilon \nu \circ \varsigma,-\mu \varepsilon v \eta,-\mu \varepsilon \nu \circ \nu$ to the present stem of the verb. Also the future act. and middle participles may be made by adding the same endings to the future stem of a verb. (See 209, 215, 216, 218.)

240. The participle is a verbal adjective. It is both verb and adjective at the same time.

I. Being an adjective, the participle is declined in gender, number, and case; it agrees in gender, number and case with the substantive that it modi. fies; like other adjectives it is either attributive or predicate; and with the article it is used as a substantive.

2. Being a verb also, the participle has voice and tense; governs the cases that the verb takes; and like other verbs it has adverbial modifiers (adjuncts).

241. The participle has not time in itself. Time with the participle is purely relative; it gets its time from the verb with which it is used.

242. Tense in the participle expresses "kind of action": the present part., durative action; the aorist participle, punctiliar action. 
243. The participle has no personal endings and is therefore not limited by a subject. The participle has no subject. It makes no affirmation and is not a mode.

244. Note the simple attributive use of the participle with a substantive:

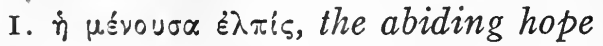

2. $\delta \ddot{\alpha} \theta p \omega \pi \circ \varsigma \delta \lambda_{\varepsilon}^{\varepsilon} \gamma \omega \nu \tau \alpha \tilde{u} \tau \alpha$, the man saying these things, (the man who says these things).

245. Examine carefully the following:

I. $\delta \pi \sigma \tau \varepsilon \dot{\omega} \omega \nu$, the one believing, he who believes

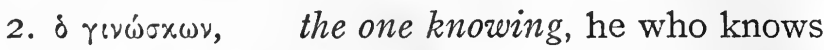

3. $\delta$ ¿xovónsvos, the one being heard, he who is heard

4. $\delta$ xpเvónevos, the one being judged, he who is judged

5. $\delta \delta \varepsilon \delta^{\prime} \mu \varepsilon v o s$, the one receiving, he who receives

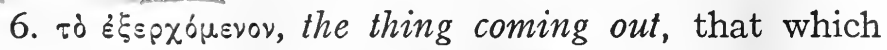
comes out

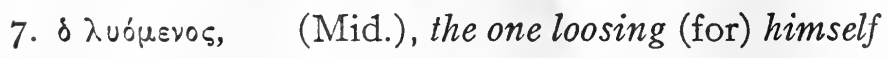

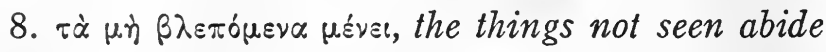

The article and participle in this use are practically equivalent to a relative clause, though not actually equivalent. The article and participle may be in any case: e.g.,

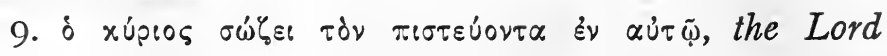
saves the one believing (him who believes) on Him.

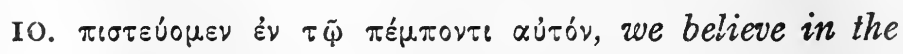
one sending (him who sends) him.

These examples practically cover the attributive use of the participle. 
246. The negative used with the participle is generally $\mu \dot{n}$, not.

247.

EXERCISES

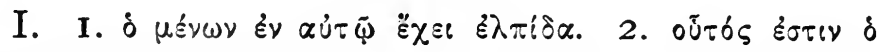

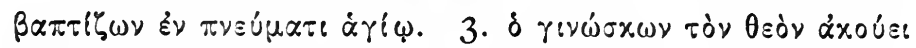

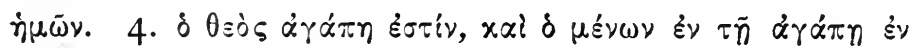

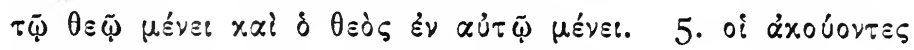
ह̇ंi

II. I. He who receives us receives Him. 2. Let us believe on him who raises the dead. 3. This is he who takes away the sins of the world. 4. He who has grace remains in hope. 5. That day we shall see him who comes in the name of the Lord.

\section{LESSON XXXI}

Participles: The Second Aorist Active and Middle 218. VOCABULARY

$\dot{\alpha} \pi \circ \theta \alpha \nu \dot{\omega} \nu$, second aorist active participle of $\dot{\alpha} \pi \circ \theta \nu \dot{\sigma} \sigma \times \omega$. $\beta \alpha \lambda \dot{\omega} \nu, \quad$ second aorist active participle of $\beta \dot{\alpha} \lambda \lambda \omega$. $\gamma \varepsilon \nu o ́ \mu \varepsilon v 0 \varsigma$, second aorist middle participle of $\gamma^{\prime}(\nu \circ \mu \alpha$. $\varepsilon i \pi \dot{\omega} v$ second aorist active participle; no present stem; $\lambda \hat{\varepsilon} \gamma \omega$ used in present.

$\varepsilon \lambda \theta \omega \dot{\omega}, \quad$ second aorist active participle; no present

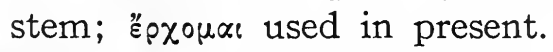

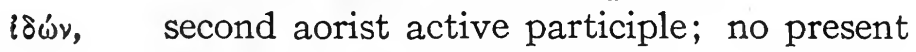
stem; $\delta \rho \alpha \dot{\omega} \omega$ used in present.

$\lambda \alpha \beta \omega \nu, \quad$ second aorist active participle of $\lambda \alpha \mu \beta \alpha^{\prime} \nu(\nu$.

249. It will be seen from the vocabulary that the second aorist active and middle participles of the 
thematic vowel type have the same endings as the present active and middle participles, $-\omega \nu$ and $-\mu \varepsilon v \circ \varsigma$.

250. The declension of $\lambda \alpha \beta \omega \nu,-0 \tilde{\sigma} \sigma \alpha,-6 \nu$, the second aorist active participle of $\lambda \alpha \mu \beta \alpha \dot{\alpha} \nu \omega$, is:

\section{STEM $\lambda \alpha \beta$ $\alpha \tau-$}

\section{Singular}

Masc.

Nom. $\quad \lambda \alpha \beta \dot{\omega} \nu$

Gen. $\quad \lambda \alpha \beta \delta \nu \tau \sigma s$

Fem.

Neut.

Abl. $\quad \lambda \alpha \beta 6$ vंos

$\lambda \alpha \beta \circ \tilde{\nu} \sigma \alpha$

$\lambda \alpha \beta$ oúns

$\lambda \alpha \beta o ́ v$

Loc. $\quad \lambda \alpha \beta 6 v \tau i$

$\lambda \alpha \beta o u ́ n s$

$\lambda \alpha \beta o ́ v \tau o s$

Ins.

$\lambda \alpha \beta \delta v \tau \varepsilon$

$\lambda \alpha \beta o u ́ o n$

$\lambda \alpha \beta o ́ v \tau 0 s$

Dat. $\quad \lambda \alpha \beta \delta v \tau \varepsilon$

$\lambda \alpha \beta o u ́ n$

$\lambda \alpha \beta o ́ v \tau$.

Acc. $\quad \lambda \alpha \beta \delta v \tau \alpha$

$\lambda \alpha \beta o u ́ n$

$\lambda \alpha \beta$ óvel

$\lambda \alpha \beta \delta \nu \tau$

$\lambda \alpha \beta \circ \tilde{u} \sigma \alpha \nu$

$\lambda \alpha \beta \delta \nu$

\section{Plural}

Masc.

Fem.

Neut.

Nom. $\lambda \alpha \beta \delta \nu \tau \varepsilon s$

$\lambda \alpha \beta \circ \tilde{\sigma} \sigma \alpha t$

$\lambda \alpha \beta o ́ v \tau \alpha$

Gen. $\quad \lambda \alpha \beta \delta \nu \tau \omega \nu$

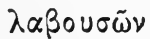

$\lambda \alpha \beta \delta \gamma \tau \omega \nu$

Abl. $\quad \lambda \alpha \beta \delta$ $\nu \tau \omega \nu$

$\lambda \alpha \beta 0 v \sigma \omega \tilde{\omega}$

$\lambda \alpha \beta o ́ v \tau \omega \nu$

Loc.

$\lambda \alpha \beta$ órals

$\lambda \alpha \beta$ บ̃ot

Ins. $\quad \lambda \alpha \beta o \tilde{u} \sigma$

$\lambda \alpha \beta o u ́ \sigma \alpha i s$

$\lambda \alpha \beta$ บ̃ot

Dat. $\quad \lambda \alpha \beta$ ṽ

$\lambda \alpha \beta o u ́ \sigma \alpha i s$

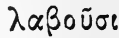

Acc. $\quad \lambda \alpha \beta o ́ v \tau \alpha \varsigma$

$\lambda \alpha \beta 0 u ́ \sigma \alpha s$

$\lambda \alpha \beta o ́ v \tau \alpha$

251. Observe: I. The second aorist active participle of the thematic vowel type is declined like the present

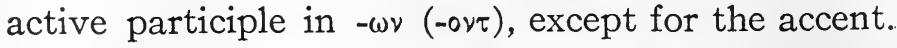
2. It is formed on the second aorist stem, and has no augment.

252. The second aorist middle participle ( $\lambda \alpha \beta \delta^{\prime} \mu \varepsilon v o s$, $-\mu \varepsilon \dot{\nu} \eta,-\mu \varepsilon v \circ v)$ is declined exactly like the present 
middle participle. The difference in form lies in the stem: $\lambda \alpha \beta \delta \mu \varepsilon v \circ \varsigma$ (stem $\lambda \alpha \beta-)$, aorist middle participle; $\lambda \alpha \mu \beta \alpha \nu o ́ \mu \varepsilon v \circ \varsigma$ (stem $\lambda \alpha \mu \beta \alpha \nu-)$, present middle participle.

253. The aorist participle is used attributively with the article, as is the present participle $(244,245)$. The difference in meaning is that the present expresses durative action and the aorist, punctiliar action.

$\delta \lambda \alpha \mu \beta \alpha^{\prime} \nu \omega \nu$, the one receiving, he who receives

$\delta \lambda \alpha \beta \omega \dot{ }$, the one having received, he who received

$\delta$ rivóusvos, the one becoming, he who becomes

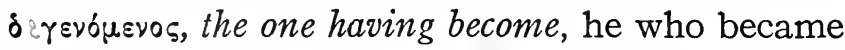

254. All participles may be used in the predicate. Study these examples carefully:

I. $\varepsilon i \pi \dot{\omega} \nu \tau \alpha \tilde{u} \tau \alpha \dot{\alpha} \pi \tilde{\eta} \lambda \theta \varepsilon v$,
a. Having said this
b. When he said this
c. After he said this
he went away
d. He said this and

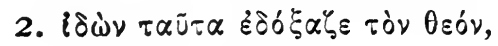
a. Seeing this
b. When he saw this $\}$ he was glorifying God
c. Because he saw this

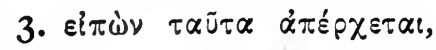
a. Having said this
b. After he said this $\}$ he goes away.

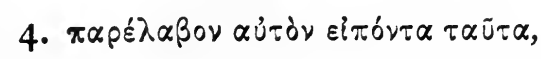
They received him
$\left\{\begin{array}{l}\text { when he said this, } \\ \text { after he said this, } \\ \text { because he said this, }\end{array}\right.$ 


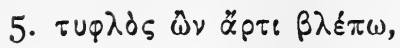

$\left.\begin{array}{l}\text { a. Being blind } \\ \text { b. Whereas I was blind }\end{array}\right\}$ now I see

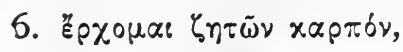

$$
\text { I come }\left\{\begin{array}{l}
\text { seeking fruit, } a . \\
\text { to seek fruit, } \quad b .
\end{array}\right.
$$

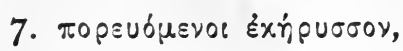

a. Advancing

$\left.\begin{array}{l}\text { b. As they were advancing } \\ \text { c. While they were advancing }\end{array}\right\}$ they were announcing

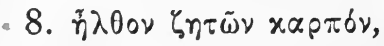

$$
\text { I came }\left\{\begin{array}{l}
\text { seeking fruit, } a . \\
\text { to seek fruit, } b .
\end{array}\right.
$$

9. $\dot{\pi} \pi \lambda \alpha \beta \dot{\omega} \nu \varepsilon i \pi \varepsilon \nu$, answering (catching up in speech) he said.

255. From the examples given above it is to be observed that:

I. The action of the present participle may precede (antecedent, Ex. 5), coincide with (simultaneous, Ex. 7), or follow (subsequent, Ex. 8 and 6) the action of the principal verb.

2. The action of the aorist participle may be antecedent to (Ex. I, $2 b, c, 3$ and 4 ), or simultaneous with (Ex. $2 a, 9)$, that of the principal verb.

The aorist participle does not express subsequent action, although it may be used proleptically.

Whether the action expressed by a participle is antecedent, simultaneous, or subsequent to that of the principal verb must be determined from the context. 
256. The participles in the examples under 254 are varieties of what is called the circumstantial participle. The circumstantial participle is practically an additional statement added more or less loosely to the verbal notion of the principal verb. It may agree with the subject or object of the principa? verb, or with any other substantive or pronoun in the sentence (see 254,4 ). The participle in itself does not express time, manner, cause, purpose, etc., as suggested in the translation of the examples in 254 . These ideas are not in the participle, but are suggested by the context.

257.

\section{ExERCISES}

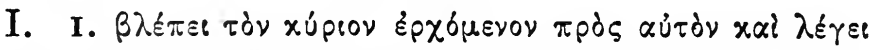

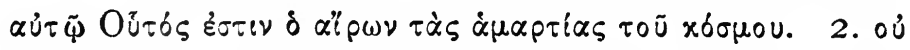

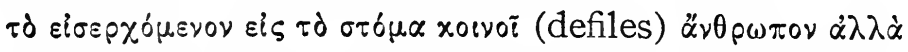

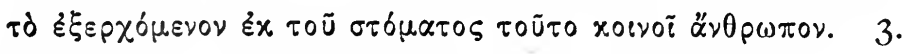

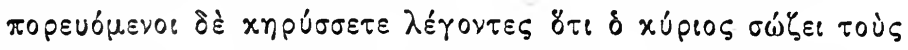

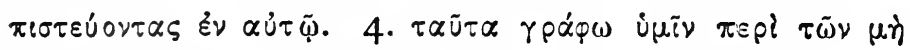

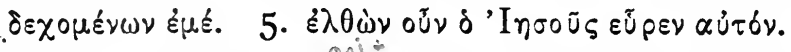

II. I. He rejoices, saying that he saw the spirit coming upon $(\dot{\varepsilon} \pi i)^{1}$ him. 2. We saw him while he was teaching in the temple. 3. The Lord said to those who were coming to him that God hears those believing on Him. 4. When he saw the child he went away. 5. Not having received the promises they died.

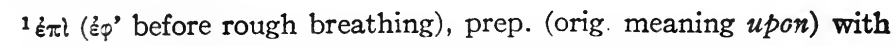
gen., upon, at, by; with loc., upon, on, over; with acc., upon, over. 


\section{LESSON XXXII}

Third Declension (Continued): Mutes and Liquids 258. VOCABULARY

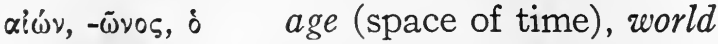

$$
\begin{aligned}
& \dot{\alpha} \mu \pi \varepsilon \lambda \dot{\omega} \nu,-\tilde{\omega} \nu \circ, \delta \text {, vineyard } \\
& \dot{\eta} \gamma \varepsilon \mu \omega \dot{\nu}, \text {-óvos, } \dot{\delta}, \quad l e a d e r \text {, governor } \\
& \mu \dot{\alpha} \sigma \tau \iota \xi,-\imath \gamma \circ \varsigma, \dot{\eta}, \quad w h i p, \text { scourge, plague } \\
& \pi \circ \mu \eta \dot{\eta} v, \text {-हैvos, } \delta \text {, shepherd } \\
& \sigma \alpha \dot{\rho} \xi, \sigma \alpha \rho x \delta \sigma, \dot{\eta}, \quad \text { flesh } \\
& \sigma \alpha \dot{\lambda} \lambda \tau \gamma \xi,-\imath \gamma \gamma \circ \varsigma, \dot{\eta}, \text { trumpet }
\end{aligned}
$$

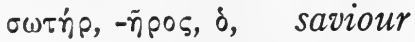

$$
\begin{aligned}
& \chi \varepsilon i \rho, \chi \varepsilon i \rho o ́ s, \dot{\eta}, \quad \text { hand }
\end{aligned}
$$

259. The declension of $\sigma \alpha \dot{\rho} \xi \xi$ and $\mu \alpha \dot{\sigma} \tau \iota \xi$ :

STEM $\sigma \alpha \rho x-$

Nom. $\sigma \alpha \dot{\rho} \xi \xi$

Gen. oxpxós

Ab1. oxpxós

Loc. $\sigma \alpha \rho x i$

Ins. $\quad$ expxí

Dat. $\sigma \alpha \rho x i$

Acc. $\sigma \alpha \dot{\rho} \rho \alpha$

Nom. $\sigma \alpha \dot{\rho} \rho x \varepsilon s$

Gen. $\sigma \alpha \rho x \tilde{\omega} \nu$

Ab1. $\quad \sigma \alpha \rho x \cdot \bar{\omega} \nu$

Loc. $\sigma \alpha \rho \xi \xi$

Ins. $\quad \sigma \alpha \rho \xi \xi$

Dat. $\sigma \alpha \rho \xi i$

Acc. $\sigma \alpha \dot{\alpha} p \alpha \varsigma$
Singular

STEM $\mu \alpha \sigma \tau l \gamma-$

Nom. $\mu \alpha \dot{\sigma \tau t \xi}$

Gen. $\mu \alpha \dot{\sigma \tau t \gamma o s}$

Abl. $\mu \dot{\alpha} \sigma \tau i \gamma \circ s$

Loc. $\mu \alpha \dot{\sigma \tau t \gamma s}$

Ins. $\quad \mu \alpha \dot{\sigma} \sigma \tau, \gamma$

Dat. $\quad \mu \dot{\alpha} \sigma \tau \imath \gamma \iota$

Acc. $\quad \mu \alpha \dot{\sigma} \sigma \tau \imath \alpha \alpha$

Plural

Nom. $\mu \dot{\alpha} \sigma \tau t \gamma \in \varsigma$

Gen. $\quad \mu \alpha \sigma \tau i \gamma \omega \nu$

Ab1. $\mu \alpha \sigma \tau i \gamma \omega \nu$

Loc. $\mu \alpha \dot{\sigma} \tau \iota \xi$

Ins. $\mu \alpha \dot{\sigma} \sigma \tau ! \xi \iota$

Dat. $\quad \mu \alpha \dot{\sigma} \sigma: \xi_{\ell}$

Acc. $\mu \dot{\alpha} \sigma \tau เ \gamma \alpha \varsigma$ 


\section{THIRD DECLENSION — CONTINUED 107}

260. Observe: I. The stems of $\sigma \alpha \dot{\rho} \xi$ and $\mu \alpha \dot{\sigma} \sigma \tau \xi$ end in palatal mutes $(2 \mathrm{I} 7,2)$. 2. The nominative sing. is formed by adding $s$ to the stem. For the changes that occur with $\varsigma$ and a palatal mute, see 2 I8. 3. The accent of $\sigma \alpha \rho \xi$ is according to the rule stated in 226 .

261. A few substantives with stems in a labial mute ( $\pi$ or $\beta$ ) occur in the New Testament. (The nom. is formed by adding $\varsigma$ to the stem and then ends in $-\psi$, see 218 ).

ExAmples: $\lambda\left(\dot{i}, \lambda_{i} \beta 6 \varsigma, \delta\right.$, the $S . W$. wind ox6 $\lambda \circ \psi,-0 \pi \circ \varsigma, \delta$, a stake, a thorn

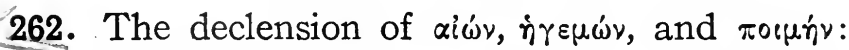

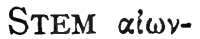

STEM $\dot{\gamma} \gamma \varepsilon \mu \mathrm{\nu}-$ Singular

Nom $\quad \alpha i \omega \nu$

Gen. aī̄vos

A.b1. aī̄vos

Loc. $\alpha i \tilde{\omega} v t$

Ins. $\alpha i \tilde{\omega} v t$

Dat. $\alpha i \tilde{\nu} v$

Acc. $\alpha i \tilde{\omega} \nu \alpha$

Nom. aī̄ves

Gen. $\quad \alpha i \omega \dot{\nu} \omega \nu$

Abl. $\quad \alpha i \omega \nu \omega \nu$

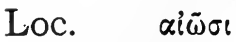

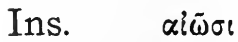

Dat. $\alpha i \omega \bar{\sigma}$

Acc. aī̄vas

$\dot{\eta} \gamma \varepsilon \mu \dot{\omega} \nu$

$\dot{\eta} \gamma \varepsilon \mu \dot{o}^{\prime} \mathrm{OS}$

$\dot{\eta} \gamma \varepsilon \mu \dot{o}$ Vos

$\dot{\eta} \gamma \varepsilon \mu o ́ v e$

$\dot{\eta} \gamma \varepsilon \mu \dot{v} v t$

$\dot{\eta} \gamma \varepsilon \mu b v \mathrm{~s}$

ìrefóva

Plural $a$. Stems in $-\nu$ are sometimes called nasal stems.

\begin{tabular}{|c|}
\hline \\
\hline 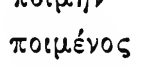 \\
\hline 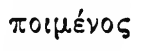 \\
\hline 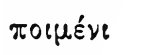 \\
\hline 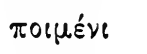 \\
\hline$\pi 0 \% \mu \varepsilon ́ v t$ \\
\hline 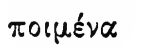 \\
\hline
\end{tabular}

$\pi \circ \leftrightarrow \mu \varepsilon v \omega \nu$

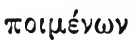

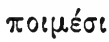

$\pi 01 \mu \varepsilon \dot{\sigma e t}$

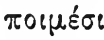

$\pi \circ \mu \varepsilon ́ v a \varsigma$

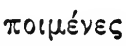


263. Observe: I. The nominative is formed from the mere stem. In the case of aiws the stem vowel is unchanged; in the case of $\dot{\eta} \gamma \varepsilon \mu \omega \dot{\omega} \nu$ and $\pi \circ \mu \dot{\eta} \nu$ the short stem vowel is lengthened (formative lengthening), $\circ$ to $\omega$, and $\varepsilon$ to $\eta$. 2. In the loc., ins., and dat. plural the $-y$ - of the stem drops out, without any change in the stem vowel.

264. The declension of $\sigma \omega \tau \dot{p} p$ :

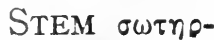

Singular

Nom. $\sigma \omega \tau \dot{n} p$

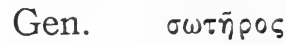

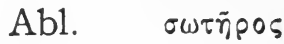

Loc. $\quad \sigma \omega \tau \tilde{\eta} p$.

Ins. $\quad \sigma \omega \tau \tilde{\eta} \rho t$

Dat. $\sigma \omega \tau \tilde{\eta} p t$

Acc. $\sigma \omega \tau \tilde{\eta} \rho \alpha$
Plural

$\sigma \omega \tau \tilde{\eta} \rho \varepsilon \varsigma$

$\sigma \omega \tau \dot{n} p \omega \nu$

$\sigma \omega \tau \dot{n} p \omega \nu$

$\sigma \omega \tau \tilde{r} p \sigma s$

$\sigma \omega \tau \tilde{\eta} p \sigma \ell$

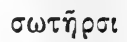

$\sigma \omega \tau \tilde{\eta} p \alpha \varsigma$

Observe: I. The nominative is made from the stem without any change. 2. In the loc., ins., and dat. plural the ending - $\sigma$ is added to the stem without any change of the stem vowel or consonant.

a. $\chi s i \rho$, gen. $\chi s ı \rho o ́ s$, is declined regularly except in the loc. inst., and dat. plur., which have $\chi \varepsilon p o$ i.

The real stem of $\chi$ sip is $\chi \varepsilon \rho \sigma-$.

265. A circumstantial participle (generally present or aorist) may be used in the genitive ${ }^{1}$ case to agree with a substantive or pronoun in a construction grammatically independent of the rest of the sen-

${ }^{1}$ In fact the case may be either genitive or ablative. 
tence. This construction is called the genitive absolute. Thus:

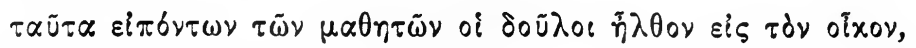
the disciples having said this $\}$ the servants went after (or when) the disciples said this $\}$ into the house

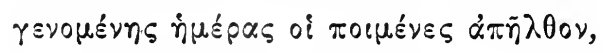
$\left.\begin{array}{l}\text { day having come } \\ \text { when day came }\end{array}\right\}$ the shepherds went away

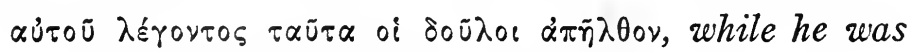
saying this the servants departed

a. The genitive absolute is found also when the participle could have agreed with some substantive or pronoun in the sentence. $b$. Sometimes the genitive absolute is used without a substantive or pronounthe participle alone.

266.

\section{EXERCISES}

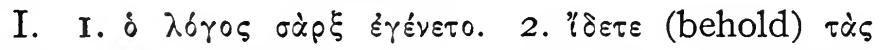

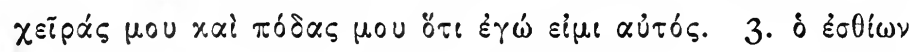

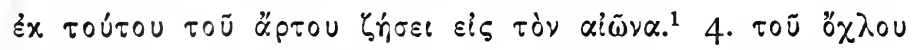

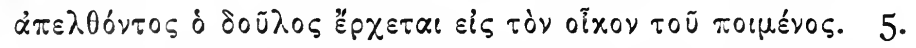

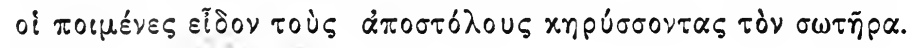

II. I. Truth abides forever. 2. Behold his hands and feet. 3. I write these (things) with my hand. 4. The Saviour having departed, the crowd said this. 5. While he was going away he saw an angel.

1 eis tòv a'īva, (into the age), forever. 


\section{LESSON XXXIII}

Third Deciension (Continued): Liquid Stems in $-\varepsilon p$ (Syncopated). The Relative Pronoun

267.

VOCABULARY

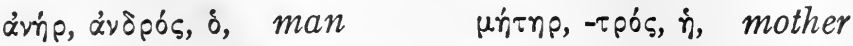

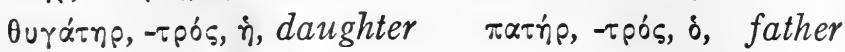

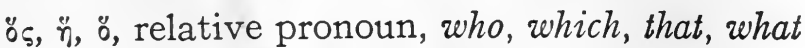

268. The declension of $\pi \alpha r_{n} p$ and $\alpha \dot{v} \dot{p}$ :

STEM $\pi \alpha \tau \varepsilon p-$

\section{Singular}

Nom. $\quad \pi \alpha \tau \dot{\eta} p$

Gen. $\quad \pi \alpha \tau p o ́ s$

$\mathrm{Abl} . \quad \pi \alpha \tau p o s$

Loc. $\pi \alpha \tau \rho !$

Ins. $\quad \pi \alpha \tau \rho^{\prime}$

Dat. $\quad \pi \alpha \tau \rho$ ?

Acc. $\quad \pi \alpha \tau \varepsilon \dot{\varepsilon} \rho \alpha$

Voc. $\quad \pi \dot{\alpha} \tau \varepsilon p$
STEM $\alpha \dot{\nu} \varepsilon \rho-$

Ncm. ávinp

Gen. àvopós

Ab1. àvopós

Loc. $\quad \alpha \dot{\alpha} \delta \rho i$

Ins. $\quad \alpha$ aspí

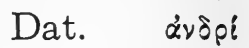

Acc. $\ddot{\nu} \nu \delta \rho$

Voc. ävep

\section{Plural}

N. V. $\pi \alpha \tau \varepsilon \dot{p} \varepsilon \varepsilon$

Gen. $\quad \pi \alpha \tau \varepsilon \rho \omega \nu$

Ab1. $\pi \alpha \tau \varepsilon \dot{\rho} \rho \omega \nu$

Loc. $\quad \pi \alpha \tau \rho \alpha \dot{\sigma} \sigma$.

Ins. $\quad \pi \alpha \tau \rho \alpha \dot{\sigma \sigma}$

Dat. $\quad \pi \alpha \tau \rho \alpha \dot{\sigma}$

Acc. $\quad \pi \alpha \tau \varepsilon \dot{\varepsilon} \rho \alpha$

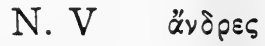

Gen. $\quad \alpha \dot{\nu} \rho \tilde{\omega} \nu$

$\mathrm{Ab1}$ a $\quad \alpha \delta \rho \tilde{\omega} \nu$

Loc. àvopáat

Ins. $\quad \dot{\alpha} \delta \delta \rho \alpha \dot{\sigma}$

Dat. $\quad \dot{\nu} \delta \rho \alpha \dot{\sigma} \sigma$

Acc. $\quad \ddot{v \delta \rho \alpha \varsigma}$

269. Observe: I. The nom. is formed from the simple stem; and the short vowel $\varepsilon$ is lengthened (formative lengthening) to $\eta$. 


\section{RELATIVE PRONOUN}

2. Owing to the effect of the accent in the loc., ins., and dat. sing. of $\pi \alpha \tau \dot{p} p$ the vowel $\varepsilon$ of the stem is suppressed; and at the same time in the loc., ins., and dat. plural $\alpha$ is developed after $\rho$ to facilitate pronunciation (also in $\dot{\alpha} \nu \delta \rho \dot{\sigma} \sigma \iota^{1}$ ).

3. In $\dot{\alpha} \vee \dot{p} p$, when $\varepsilon$ is suppressed, there is inserted sympathetically the consonant $\delta$ for the sake of euphony.

4. The voc. sing. is the simple stem, and has recessive accent.

270. uninp, mother, and turárnp, daughter, are declined like $\pi \alpha \tau \eta^{\prime} \rho$. The vocative of $\theta u \gamma \alpha^{\prime} \tau \eta p$ is $\theta \dot{u} \gamma \alpha \tau \varepsilon p$. No voc. of $\mu$ in $\eta_{p}$ occurs in the New Testament.

271. The declension of the relative pronoun ${ }^{\circ}{ }_{s}, \ddot{\eta}, 8$ is:

\section{Singular}

Masc. Fem. Neut.

Nom. ös $\ddot{\eta}$ \%

Gen. oũ $\tilde{\eta} \varsigma$ ou

Abl. ỡ $\tilde{y} s$ oU

Loc. $\quad \tilde{n} \quad \tilde{\omega}$

Ins. $\tilde{\varphi} \quad \tilde{n} \quad \tilde{\varphi}$

Dat. $\tilde{\varphi} \quad \tilde{n} \quad \tilde{\varphi}$

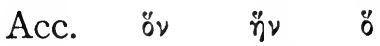

\section{Plural}

Masc. Fem. Neut.

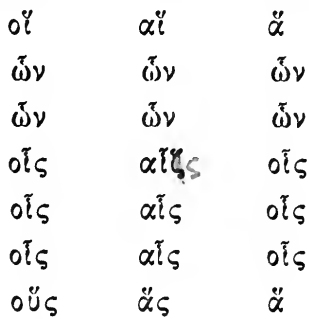

It will be noticed that this pronoun is declined in the first and second declensions. Observe that every form has the rough breathing (') and an accent.

272. The relative pronoun generally agrees with its antecedent in gender and number; but it may have

${ }^{1}-\rho \alpha$-actually represent the vocalic sound of $p$. 
its own case in its clause, or it may be attracted to the case of its antecedent.

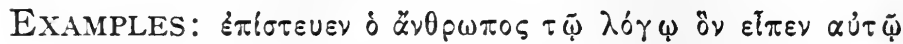
$\delta$ 'Inoous, the man was believing the word that Jesus spoke to him.

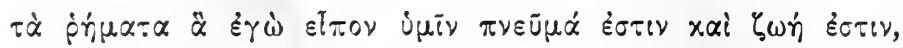
the words which I spoke to you are spirit and are life.

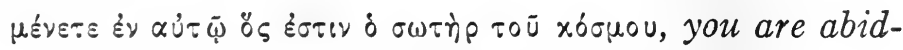
ing in him who is the Saviour of the world.

273. Usually the attraction of the relative to the case of the antecedent is from the accusative (in which it naturally would be in its own clause) to some other oblique case:

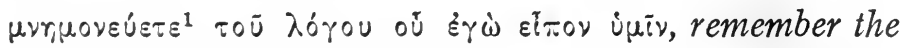
word that I said to you.

Here or has been attracted to the case of $\lambda$ 6rou. In its own clause it naturally would be in the accusative.

274. Often the relative has no antecedent expressed:

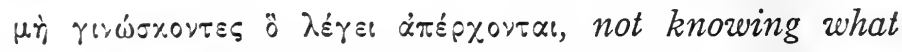
(that which) he says they go away.

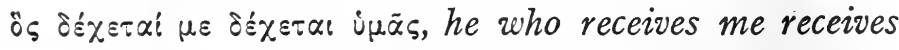
you.

275.

ExerCISES

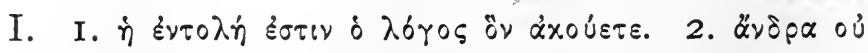

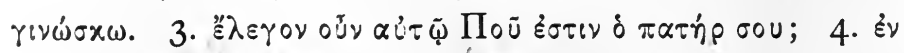
1 urnjovefo, to remember, with genitive. $\quad{ }^{2} \pi \circ \bar{u}$, where. 


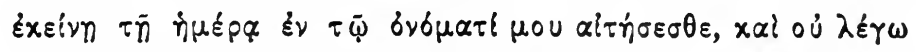

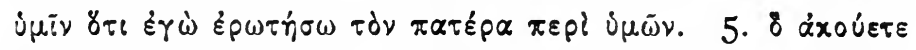

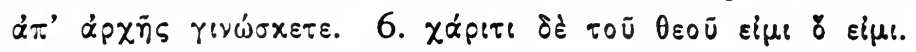

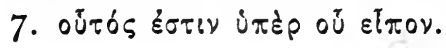

II. I. The hope which we have is eternal. 2. This is my father and my mother. 3. We believe in him who died for us. 4. He shall ask the father concerning us. 5. This is the daughter concerning whom I spoke.

\section{LESSON XXXIV}

Third Declension (Continued): Stems in :

The Interrogative and Indefinite Pronouns

276.

VOCABULARY

$\alpha^{2} \nu \alpha \dot{\sigma \tau \alpha \tau ! \varsigma,}-\varepsilon \omega \varsigma, \dot{\eta}$,

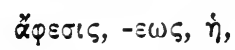

resurrection

$\gamma \gamma \tilde{\omega} \sigma ! \varsigma,-\varepsilon \omega \varsigma, \dot{\eta}$, remission, forgiveness

$\delta \dot{y} v \mu(\varsigma,-\varepsilon \omega \varsigma, \dot{\eta}, \quad$ power

$\theta \lambda(\dot{v} เ \varsigma,-\varepsilon \omega \varsigma, \dot{\eta}, \quad$ tribulation, distress

xpí:s, $-\varepsilon \omega \varsigma, \dot{\eta}, \quad$ judgment

$x \tau i \sigma เ s,-\varepsilon \omega \varsigma, \dot{\eta}, \quad$ creation

$\pi \alpha \rho \alpha \dot{x} \lambda \eta \nabla เ \varsigma,-\varepsilon \omega \varsigma, \dot{\eta}$, exhortation

$\pi i \sigma \tau เ \varsigma,-\varepsilon \omega \varsigma, \dot{\eta}, \quad$ faith

$\pi \delta \lambda_{\imath} \varsigma,-\varepsilon \omega \varsigma, \dot{\eta}, \quad$ city

$\tau i \varsigma, \tau i$, interrogative pronoun, who, which, what?

$\tau ! \varsigma, \tau$, indefinite pronoun, one, a certain one. a certain

thing; some one, something.

жо , interrogative adverb, where? 
277. The declension of $\pi 6 \lambda_{\imath \varsigma}$ is:

$$
\text { STEM } \pi 0 \lambda_{l-}\left(\pi 0 \lambda_{\varepsilon l-}\right)
$$

\begin{tabular}{|c|c|c|}
\hline \multicolumn{2}{|c|}{ Singular } & Plural \\
\hline Nom. & $\pi \delta \lambda_{l \varsigma}$ & $\pi \delta \lambda \varepsilon \iota \varsigma \varsigma$ \\
\hline Gen. & $\pi \delta \hat{\lambda} \varepsilon \omega \varsigma$ & $\pi 6 \lambda \varepsilon \omega \nu$ \\
\hline Abl. & $\pi \delta \hat{\lambda \varepsilon \omega \varsigma}$ & $\pi \delta \lambda_{\varepsilon \omega \nu}$ \\
\hline Loc. & $\pi \delta \lambda \varepsilon \varepsilon$ & $\pi \delta \lambda \varepsilon \sigma \sigma \iota$ \\
\hline Ins. & $\pi \delta \lambda \varepsilon \varepsilon$ & $\pi \delta \lambda_{\varepsilon \sigma \iota}$ \\
\hline Dat. & $\pi \delta \lambda \varepsilon \ell$ & $\pi 6 \lambda \varepsilon \sigma \sigma \iota$ \\
\hline Acc. & $\pi \delta \lambda_{\iota \nu}$ & $\pi \delta ́ \lambda \varepsilon \iota \varsigma$ \\
\hline
\end{tabular}

The voc. sing. $\pi \delta \lambda_{l}$ does not occur in the New Testament.

278. Observe: 1 . In the nom. and acc. sing. the stem is $\pi 0 \lambda_{l-} .2$. In the other cases the stem is $\pi 0 \lambda \varepsilon t$ ( $c$ is here a semi-vowel). 3. $\pi \delta \lambda^{\prime} \varepsilon \omega \varsigma$ and $\pi \delta \lambda_{\varepsilon \omega \nu}$ of the gen. and abl. are for $\pi 0 \lambda \varepsilon(\iota) \omega s$ and $\pi 0 \lambda \varepsilon(\iota) \omega \nu$. $\varepsilon$ here preserves the semivowel sound of $\imath$ which is dropped. Note the apparent exception to rule of accent, that the accent cannot stand on antepenult when the ultima is long. $-\omega \varsigma$ is lengthened from -os. 4. In the loc., ins., and dat. sing. $\pi \delta^{\lambda} \varepsilon_{\varepsilon}$ is for $\pi \dot{\delta} \lambda \varepsilon(\iota) \iota$; ? of the case ending and $\varepsilon$ contract, $\varepsilon+\imath=\varepsilon$ (diphthong). 5. In acc. sing. $-\nu$ is the case ending, not $-\alpha$. 6. The nom. plural $\pi \dot{\delta} \lambda \varepsilon เ \varsigma$ is for $\pi 0 \lambda \varepsilon(\iota) \varepsilon \varsigma ; \varepsilon+\varepsilon$ contract tc sı (diphthong). 7. The acc. plural (old form $\pi \dot{\delta} \lambda \varepsilon \alpha \varsigma)$ is assimilated to the nominative.

279. Like $\pi \hat{\lambda} \lambda_{\iota \varsigma}$ are declined all substantives (not having accent on ultima) in are mainly abstract substantives of the feminine gender. 
280. The declension of the interrogative pronoun $\tau i \xi, \tau i$ is:

Singular

Masc.and Fem. Neut. Masc. and Fem. Neut.

Nom. tis

Gen. Tivos

Abl. Tivos

Loc. Tive

Ins. Tive

Dat. Tive

Acc. tiva $\tau i$

tivos

tivos

tive

$\tau$ tre

tive

$\tau \dot{\imath}$ тives

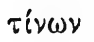

$\tau i \nu \omega \nu$

$\tau i \sigma \iota$

tío

Tíol

Tivas
Plural

riva

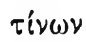

$\tau i v \omega \nu$

$\tau i \sigma \ell$

tíol

$\tau i \sigma$ b

Tives

$a$. Note that this pronoun is declined in the third declension; the masculine and feminine genders are alike; the neuter differs from the masculine and feminine only in the nom. and acc. $b$. The interrogative pronoun has the acute accent on the first syllable; it is never changed to the grave.

281. The declension of the indefinite pronoun $\tau i \varsigma, \tau i$ is :

\section{Singular}

Plural

Masc. and Fem. Neut. Masc. and Fem. Neut.

\begin{tabular}{|c|c|c|c|c|}
\hline Nom. & $\tau ! \zeta$ & $\tau i$ & $\tau \iota \nu \varepsilon ́ \varsigma$ & $\tau \in v \alpha^{\prime}$ \\
\hline Gen. & 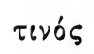 & 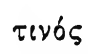 & $\tau \iota \nu \tilde{\omega \nu}$ & $\tau \iota \nu \tilde{\omega} \nu$ \\
\hline Abl. & Tivós & 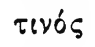 & $\tau \ell \nu \bar{\omega} \nu$ & $\tau \iota \nu \tilde{\omega} \nu$ \\
\hline Loc. & $\tau e v t$ & $\tau \iota v t$ & $\tau \iota \sigma t$ & $\tau \imath \sigma i$ \\
\hline Ins. & $\tau \iota v t^{\prime}$ & $\tau \iota v t$ & $\tau \iota \sigma i$ & $\tau \imath \sigma i$ \\
\hline Dat. & $\tau \iota \nu t$ & $\tau \iota \nu !$ & $\tau \iota \sigma i$ & $\tau \ell \sigma i$ \\
\hline Acc. & $\tau\left(\nu \alpha \alpha^{\prime}\right.$ & $\tau i$ & $\tau \iota \nu \alpha \alpha^{\prime} \varsigma$ & $\tau: \nu \alpha ́$ \\
\hline
\end{tabular}

Note that the indefinite pronoun is declined like the interrogative, except that the accent is placed on 
the ultima because it is an enclitic and loses or retains its accent according to the principles given in 138 .

282. Examine carefully the following examples of the use of the interrogative pronoun:

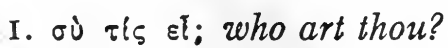

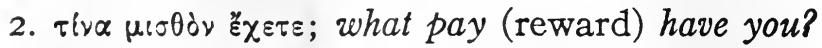

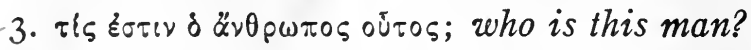

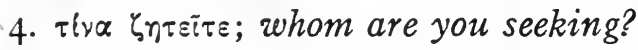

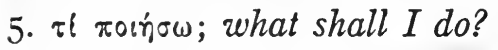

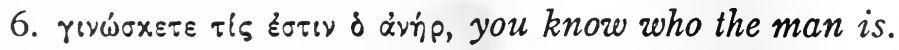

Note: I. The interrogative $\tau i c$ is used as a substantive ( 1,3 and 4 ) or as an adjective (2). 2. It is used in both direct ( $\mathbf{I}-5)$ and indirect (6) questions.

283. The neuter accusative $\tau$ is frequently used adverbially in the sense of "why."

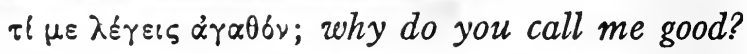

284. In indirect questions the same mode and tense is generally found as in the direct; and the same. interrogative words:

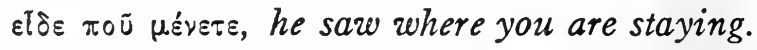

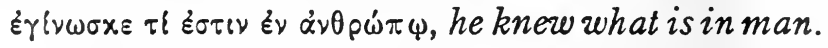

285. Study carefully the following examples of the use of the indefinite pronoun:

I. $\varepsilon i \pi \varepsilon v \tau \iota \varsigma$ $\alpha \dot{u} \tau \tilde{\varphi}$,

one (a certain man) said to him.

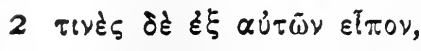
some (certain ones) of them said.

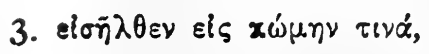
he went into a certain village. 


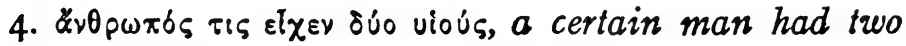
sons.

Note that the indefinite $\tau i s$ is used as a substantive ( $I$ and 2 ), or as an adjective (3 and 4 ).

286.

EXERCISES

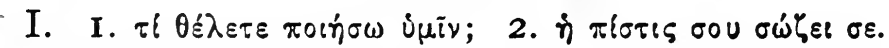

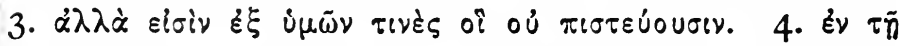

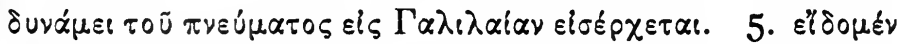

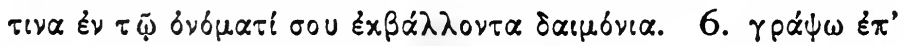

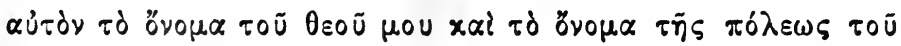
$\theta \varepsilon \circ \tilde{u} \mu \circ u^{2}$

II. I. What shall we say? 2. Ye have power to become the children of God. 3. In a certain city he was preaching the word. 4. A certain man said, "Lord, I will follow thee." 5. Why are you going away? 6. The faith which we have saves men.

\section{LESSON XXXV}

Third Declension (Continued): Stems in $-\varepsilon \cup(\varepsilon F)$ and $-\varepsilon \sigma$. 287. VOCABULARY

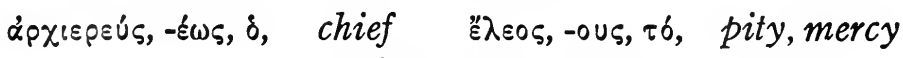

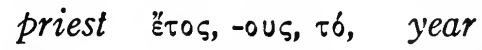

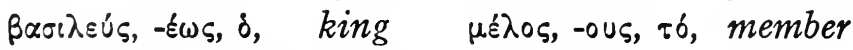

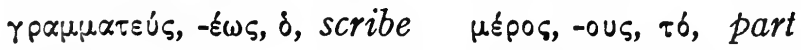

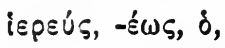

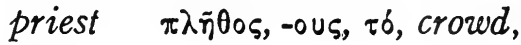
revos, -ous, $\tau \delta$, race multitude

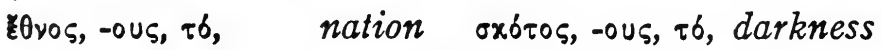

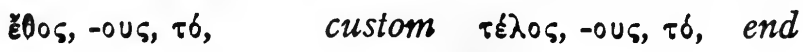


288. The declension of $\beta \alpha \sigma$ sqús is:

$$
\text { STEM } \beta \alpha \sigma i \lambda \varepsilon \cup(f)-1
$$

Singular

Nom. $\beta \alpha \sigma i \lambda \varepsilon u ́ s$

Gen. $\quad \beta \alpha \sigma \iota \lambda \varepsilon \omega \varsigma$

Abl. $\quad \beta \alpha \sigma i \lambda \varepsilon ́ \omega \varsigma$

Loc. $\quad \beta \alpha \sigma i \lambda \varepsilon i$

Ins. $\quad \beta \alpha \sigma i \lambda \varepsilon i$

Dat. $\quad \beta \alpha \sigma i \lambda \varepsilon i$

Acc. $\beta \alpha \sigma i \lambda \varepsilon ́ \alpha$

Voc. $\beta \alpha \sigma i \lambda \varepsilon \tilde{U}$
Plural

N. V. $\beta \alpha \sigma i \lambda \varepsilon i \varsigma$

$\beta \alpha \sigma i \lambda \varepsilon \omega \nu$

$\beta \alpha \sigma i \lambda \varepsilon \dot{\varepsilon} \omega \nu$

$\beta \alpha \sigma i \lambda \varepsilon \tilde{\sigma \sigma t}$

$\beta \alpha \sigma i \lambda \varepsilon \tilde{\sigma} \sigma 6$

$\beta \alpha \sigma i \lambda \varepsilon \tilde{\sigma \sigma l}$

$\beta \alpha \sigma i \lambda \varepsilon i \sigma_{5}$

Observe: I. The final $u(f)$ of the stem is dropped when it would come between two vowels; it is retained when final (vocative) or followed by a consonant (nom. sing.; loc., ins., and dat. plur.). 2. In the loc., ins., and dat. sing., and in the nom. and acc. plural contraction takes place. 3. In the acc. sing. $-\alpha$ is the case ending and not $-\nu$. The acc. plural has been assimilated to the nominative. Compare $\beta \alpha \sigma \iota \lambda \varepsilon u ́ \varsigma$ with $\pi \dot{\prime} \lambda \iota \varsigma$.

289. All substantives with nom. sing. in - $\varepsilon u ́ s$ are masculine, and are declined like $\beta a \sigma t \lambda \varepsilon u ́ s$.

290. The declension of $\gamma^{\prime}$ v'vos is:

\section{STEM $\gamma \varepsilon \vee \varepsilon \sigma-$}

\section{Singular}

Nom. révos

Gen. révous

Abl. révous

\section{Plural \\ $\gamma \varepsilon \dot{v} \eta$ \\ $\gamma \varepsilon \nu \bar{\omega} \nu(\gamma \varepsilon \nu \varepsilon \dot{\omega} \omega \nu)$ \\ $\gamma \varepsilon \nu \tilde{\omega} \nu(\gamma \varepsilon \nu \varepsilon \hat{\omega} \omega \nu)$}

${ }^{1} \mathrm{~F}$, vau, called also digamma, an old letter standing in the alphabet after $\varepsilon$, and pronounced like $w$. Its presence as a semi-vowel is often shown by $u$. 


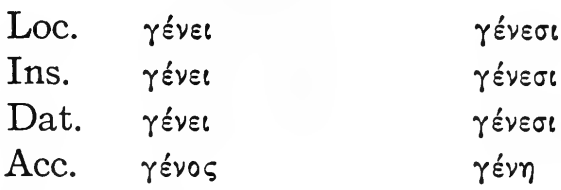

Observe: I. The nom. (and acc.) is the stem with vowel $\varepsilon$ strengthened to 0 . 2. In all other cases the $\sigma$ of the stem is dropped, and contraction of the concurrent vowels takes place.

$a$. In the gen. and abl. sing. révous came from $\gamma^{\varepsilon} v \varepsilon \sigma \circ \varsigma ; \sigma$ dropped out, $\varepsilon+\circ$ contracted to ou. $b$. In

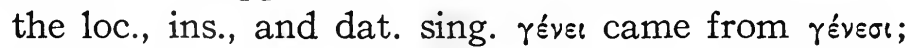
$\sigma$ dropped out, $\varepsilon+\imath$ contracted to $-\varepsilon \iota$. $c$. The nom. and acc. plural $\gamma^{\prime} \varepsilon \eta \eta$ came from $\gamma^{\varepsilon} v \varepsilon \sigma \alpha ; \sigma$ dropped out, $\varepsilon+\alpha$ contracted to $\eta$. $d$. In the gen. and abl. plural $\gamma \varepsilon \nu \tilde{\omega} \nu$ ( $\gamma \varepsilon \nu \varepsilon \dot{\omega} \omega \nu$ ) came from $\gamma \varepsilon \nu \varepsilon \dot{\sigma} \omega \nu ; \sigma$ dropped out, $\varepsilon+\omega$ contracted to $\omega$, or remained uncontracted as $\gamma \varepsilon v \varepsilon \varepsilon^{\omega} \nu$. 3. The accent of the contracted gen. and abl. plural is a circumflex over the ultima.

291. Like révos are declined all neuters with nom. sing. in -0 s (stem $-\varepsilon \sigma)$.

292.

\section{EXERCISES}

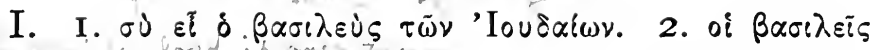

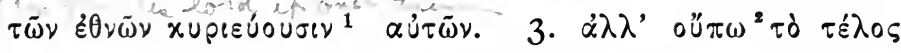

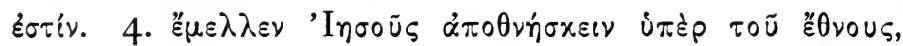

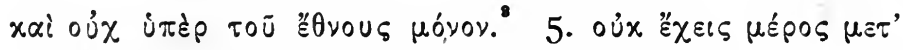

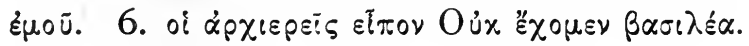

II. I. The multitude will follow him. 2. That one is not king of this world. 3. This is the gospel

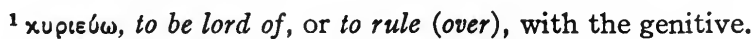

2 oür 1 , not yet.

8 uóvov. adv.. onle. 
which I proclaim among (zv) the nations. 4. You have a part in the kingdom of heaven. 5. The scribes and priests have not mercy.

\section{LESSON XXXVI}

First Aorist Indicative Active and Middle

293.

VOCABULARY

$\dot{\alpha} \gamma\left(\dot{\alpha}_{3},(1), \quad I\right.$ sanctify

$x \alpha \theta \alpha p i \zeta \omega, I$ purify

$\beta \alpha \tau \iota \lambda=\dot{\omega} \omega$, I reign, I am king $\quad x \alpha \tau \alpha \lambda \dot{u} \omega$, I destroy

$\theta \varepsilon \dot{\alpha} 0 \mu \alpha$, I behold

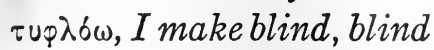

294. The second aorist tense (178) is older than the first aorist. As tense the first aorist is not a different tense from the second aorist. The second and first aorists are just two different forms of the same tense.

295. The first aorist indicative active of $\lambda \dot{v} \omega$ is:

STEM $\lambda \cup \sigma(\alpha)$ -

Singular

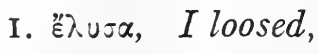

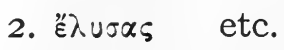
3. है

Plural

I. Ė่ $\sigma_{\sigma \alpha \mu \varepsilon \nu}$

2. $\varepsilon \lambda \cup \cup \alpha \tau \varepsilon$

3. $\stackrel{\varepsilon}{\lambda} u \sigma \alpha \nu$

First aorist active infinitive, $\lambda \tilde{\text { ũ } \alpha \ell}$

For translation of the aorist, see 187.

296. On the formation of the first aorist it is to be observed:

I. The first aorist stem is formed by adding $-\sigma \alpha$ to the verb stem.

2. The secondary active personal endings (see 66) 
are used. But $-\nu$ is not used in the first singular; and $-\varepsilon$ of the third sing. seemingly takes the place of $\alpha$.

3. In the indicative there is an augment as in the second aorist (182.3, and 70).

4. The infinitive ending is $-\alpha_{l}$; the accent is on the penult.

297. The first aorist indicative middle of $\lambda \dot{v} \omega$ is:

Singular

Plural

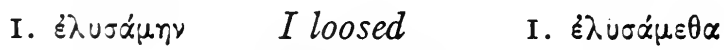

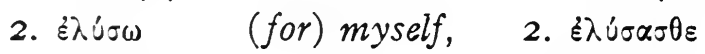

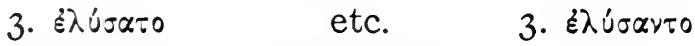

First aorist middle infinitive, $\lambda \dot{v} \sigma \alpha \sigma \theta \alpha$

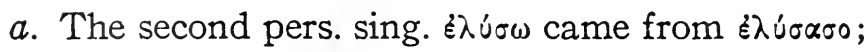
$\sigma$ dropped out, and the concurrent vowels $\alpha$ and 0 contracted to $\omega$.

298. Observe: I. That the secondary middle endings (see 80) are added directly to the theme $\lambda \cup \sigma \alpha$ -

2. The middle infinitive ending $-\sigma \theta \alpha \iota$ is added tc the theme $\lambda u \sigma \alpha-$.

299. The same principles of augment are found in the first aorist indicative as in the second aorist (182.3) and imperfect (70).

300. The form $\varepsilon \lambda \dot{v} \sigma \alpha \tau 0$ may be analyzed thus: $\dot{\varepsilon}-\lambda \dot{u}-$ $\sigma \alpha-\tau 0$; $\dot{\varepsilon}$ is the augment; $\lambda u$ - is the verb-stem, $\sigma \alpha$ is the tense suffix; $\lambda u \sigma(\alpha)$ is the tense stem, $\lambda u \sigma \alpha$ is the tense theme, and $\tau 0$ is the secondary middle personal ending of the third person. Analyze the active

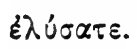

For the meaning of the aorist middle see Lesson $\mathrm{XXIV}$, and 52 . 
301. Verbs with stems ending in a vowel (except $\alpha, \varepsilon, 0$ ) regularly form the first aorist (if they have a first aorist) like $\lambda \dot{v} \omega$.

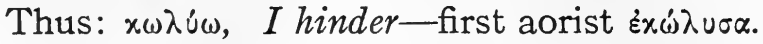
$\pi \leftarrow \tau \tau \varepsilon \dot{\omega} \omega, I$ believe-first aorist $\dot{\varepsilon} \pi i \sigma \tau \varepsilon \cup \sigma \alpha$.

302. Generally, verbs that have a first aorist do not have a second aorist; and verbs that have a second aorist do not have a first aorist.

a. A few verbs have both aorists. When they occur, the first aorist is usually transitive and the second aorist intransitive.

In the New Testament it is common for a second aorist stem to have $\alpha$ of the first aorist: thus $\varepsilon i \delta \alpha$,

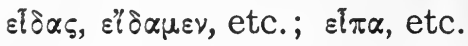

303. I. Verbs with stems ending in a short vowel $\alpha, \varepsilon$, or 0 , form the first aorist by lengthening the vowel before the tense suffix $\sigma \alpha$ (as in the future before $\sigma \% / \epsilon, 2$ I5):

$\alpha$ is lengthened to $\eta$ (except after $\varepsilon$,, , or $\rho$, when $\varepsilon$ is lengthened to $\eta \quad$ it is lengthened to $\alpha$.)

0 is lengthened to $\omega$.

Thus:

$\dot{\alpha} \gamma \alpha \pi \dot{\alpha} \omega, I$ love, $\quad$ first aorist $\dot{\eta} \gamma \dot{\alpha} \pi \eta \eta \alpha$.

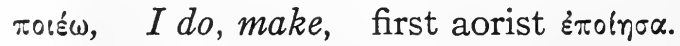

$\pi \lambda \eta \eta o ́ \omega, I ~ f i l l, \quad$ first aorist $\dot{\varepsilon} \pi \lambda \dot{n} \rho \omega \sigma \alpha$.

Active infinitives, $\dot{\alpha} \gamma \alpha \pi \tilde{\eta} \sigma \alpha l, \pi 0 \imath \tilde{\eta} \sigma \alpha l, \pi \lambda \eta \rho \tilde{\omega} \sigma \alpha l$.

2. But a few verbs like $x \alpha \lambda \varepsilon \dot{\varepsilon} \omega, I$ call, and $\tau \varepsilon \lambda \varepsilon \varepsilon \omega$, $I$ end, I complete, do not lengthen $\varepsilon$ before the aorist tense suffix. Thus:

$$
\begin{aligned}
& x \alpha \lambda \varepsilon \varepsilon \omega, I \text { call, first aorist } \varepsilon x \alpha \dot{\lambda} \lambda \varepsilon \sigma \alpha
\end{aligned}
$$

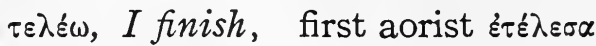


3. Verb stems withi endings in mutes make the same changes with $\sigma$ of $-\sigma \alpha$ to form the aorist, as was made with $\sigma$ of the future, 215-I6-I7.

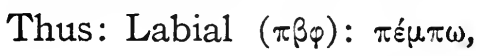
$\gamma \rho \alpha ́ \rho \omega$,

Palatal $(x \gamma \chi): \delta \iota \omega x \omega$,

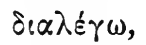

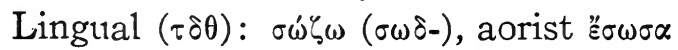
$\pi \varepsilon i \theta \omega$,

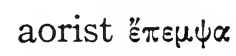
aorist "ै $\gamma \rho \alpha \psi \alpha$ aorist $\varepsilon \delta i \omega \xi \alpha$ aorist $\delta\left\llcorner\varepsilon \lambda_{\varepsilon} \xi \alpha\right.$

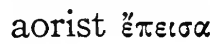

304. There is no difference in meaning between a first aorist and a second aorist. Both express punctiliar action-point action. See I78-I79.

305. There were originally two verb-types, the one denoting durative or linear action, the other momen-

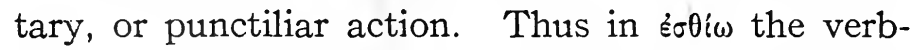
stem is durative or linear, and in "ैp $\alpha$ rov the verbstem is punctiliar. So in English "blink the eye" is a different kind of action from "live a life." In Greek this matter of the "kind of action" in the verb-stem (or root), called Aktionsart, applies to all verbs. This "kind of action" of the verb-stem itself was before there was any idea of the later tense development.

The aorist tense at first was used with verb-stems of punctiliar sense. The verb-stem itself may accent the beginning of the action, the end of the action, or the action as a whole. The aorist tense itself always means point-action (punctiliar action). But the individual verb-stem meaning may deflect the punctiliar action to the beginning or to the end. Consequently, in the aorist the tense idea is to be 
combined with the verb-stem meaning. Thus in punctiliar action three distinctions arise: (I) the unmodified point-action, called constative; (2) the point action with the stress on the beginning of the action, called ingressive; (3) the point-action with the stress on the conclusion or end of the action, called effective. Thus:

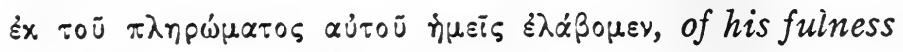
we received. (Effective.)

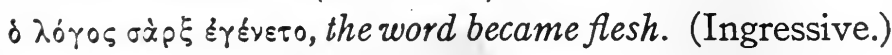

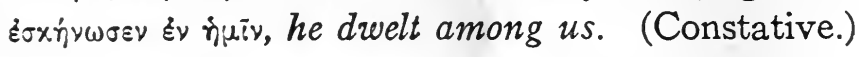

Sometimes the same word can be used for each of these ideas; as $\beta \alpha \lambda \varepsilon i \nu$ may mean "throw" (constative), or "let fly" (ingressive), or " hit" (effective).

306. The aorist tense, although at first it was confined to verbs of punctiliar action, came gradually to be made on verbs of durative action. (So also verbs of durative action came to have the tenses of punctiliar action.) Thus the tenses came to be used for the expression of the idea that once belonged only to the verb-stem (or root). That is, the aorist tense imposed a punctiliar idea on a durative verbstem. (So also the present tense imposed a durative idea on a punctiliar verb-stem.) Thus the aorist just treats as punctiliar an act which is not in itself point-action. This is the advance that the tense makes on the verb-stem (or root). So all aorists are punctiliar, in fact or statement.

307. Of course the "kind of action" of the tense (punctiliar) and the "kind of action" of the verb- 
stem (Aktionsart) run through the whole tense (modes, infinitive, and participle).

308. What was said in Lessons XXIV and XXV about the meaning of the second aorist applies also to the first aorist; and what is said in this lesson applies to the second aorist. Review the meaning of the modes.

309.

\section{EXERCISES}

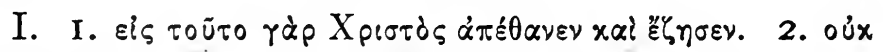

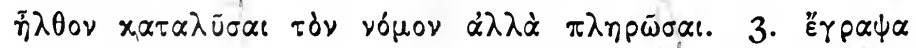

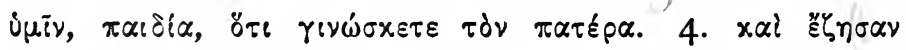

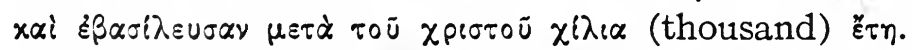

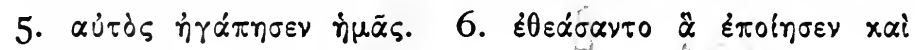

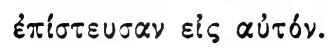

II. I. Darkness blinded his eyes. 2. We did not receive the gospel because we did not hear the word. 3. They made him king. 4. He came to destroy the works of the devil. 5. He sanctified them in truth.

\section{LESSON XXXVII}

First Aorist Subjunctive Active and Middle

310.

VOCABULARY

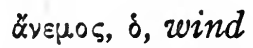
vouih(1, I think, suppose

$\theta \alpha \cup \mu \alpha \dot{\zeta}, \omega, I$ wonder, marvel $\pi \rho \varepsilon \sigma \beta u ́ \tau \varepsilon \rho \circ \varsigma, \delta$, elder

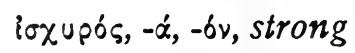

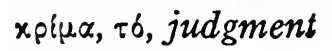

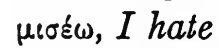
vixáw, $I$ conquer ox $\alpha \nu \delta \alpha \lambda i \zeta \omega, I$ cause to stumble, offend

Qbßos, o, fear xpsia, $\dot{\eta}$, need 
311. The first aorist subjunctive active and middle of $\lambda \dot{u} \omega$ :

\section{ACtive}

Singular

I. $\lambda u ́ \sigma \omega$

2. $\lambda$ úons

3. ưon

I. $\lambda \dot{v} \sigma \omega \mu \alpha t$

2. $\lambda \dot{v o n}$

3. $\lambda \dot{\sigma o \eta \tau \alpha t ~}$

\section{Plural}

I. $\lambda \dot{\sigma} \sigma \omega \mu \varepsilon \nu$

2. $\lambda \dot{\sigma} \sigma \eta \tau \varepsilon$

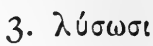

\section{MIDDLE}

I. $\lambda \cup \sigma \omega \dot{\omega} \mu \varepsilon \theta \alpha$

2. $\lambda \dot{U} \sigma \eta \sigma \theta \varepsilon$

3. $\lambda \dot{\sigma} \sigma \omega \nu \tau \alpha$ t

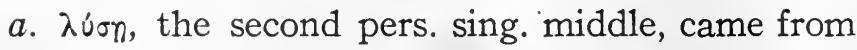
$\lambda$ von,all; $\sigma$ dropped out, and $\eta$ and $\alpha_{l}$ contracted to $n$.

312. Observe: I. There is no augment. Augment belongs to the secondary tenses of the indicative only. 2. The stem $\lambda \cup \sigma(\alpha)(\sigma(\alpha)$ is the tense suffix) is the same stem as in the aorist indicative. 3. The long thematic vowel $\omega / \eta$ is added to the stem. Compare the present subjunctive $\mathrm{I}_{5}$ and $\mathrm{I}_{72}$. 4. The personal endings are the primary active and middle endings, the same as in the present subjunctive ( 165 and 172 ).

313. In the formation of the first aorist subjunctive of verbs with stems ending in a short vowel or a mute, the same changes are made at the end of the stem as in the first aorist indicative (see 303. I, 3). Thus the first aorist subjunctive of

$$
\begin{aligned}
& \dot{\alpha} \gamma \alpha \pi \dot{\alpha} \omega \text { is } \dot{\alpha} \gamma \alpha \pi \dot{\eta} \sigma \omega, \dot{\alpha} \gamma \alpha \pi \dot{\eta} \sigma n s \text {, etc. }
\end{aligned}
$$

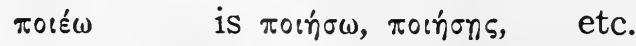

$$
\begin{aligned}
& \pi \lambda \eta p \sigma \omega \text { is } \pi \lambda \eta p \omega \dot{\omega} \sigma \omega, \pi \lambda \eta \rho \omega \sigma n s \text {, etc. }
\end{aligned}
$$




\section{FIRST AORIST SUBJUNCTIVE}

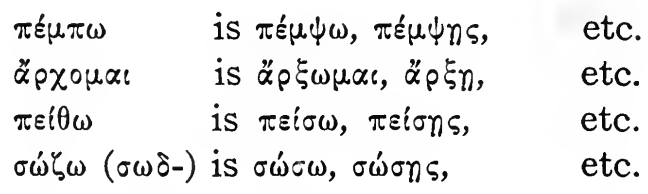

314. Write the first aorist subjunctive of

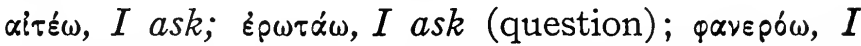

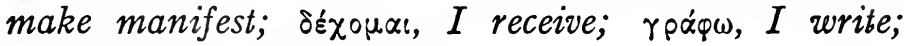
$\beta \lambda \varepsilon ́ \pi \omega, I$ see; $\sigma \omega \dot{\zeta} \omega, I$ save.

315. Of course the "kind of action" of the aorist subjunctive is punctiliar; and Aktionsart is present as in the indicative. What was said in 304-306 about punctiliar action and Aktionsart applies to the aorist subjunctive.

316. It needs to be repeated that the difference in the meaning between the present subjunctive and the aorist subjunctive is in the "kind of action" expressed by the two tenses: durative action in the present, and punctiliar action in the aorist.

317. The aorist subjunctive (in the second and third persons) with $\mu$ n is used to express a prohibition.

I. Generally in the second person:

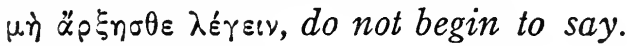

2. Less often in the third person: $\mu \hbar \tau i s$ i $\mu \tilde{\alpha} \varsigma \pi \lambda \alpha \nu \dot{n} \sigma n$, let no one cause you to err.

318.

EXERCISES

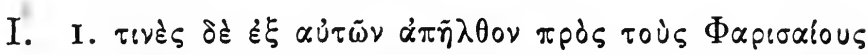

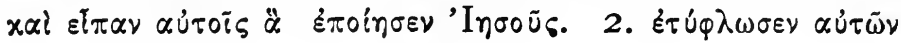




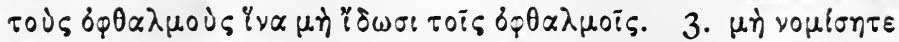

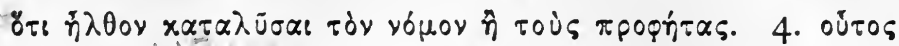

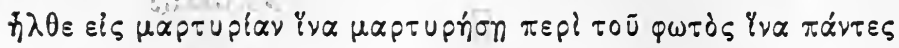

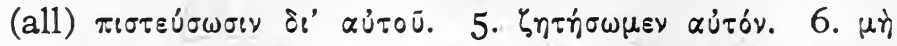
$\pi \varepsilon p l \tau 0 u ́ \tau \omega \nu$ Ypáuns.

II. I. What shall we do? 2. Let us receive the Gospel. 3. Do not love the world. 4. They asked him concerning the parable. 5. If we do his will, he will love us.

\section{LESSON XXXVIII}

First Aorist Active and Middle Participle. Adjectives of the Third Declension. Declension of $\pi \tilde{\alpha} \varsigma$

319.

VOCABULARY

$\ddot{\alpha} \delta เ x \circ s,-\eta,-o v$, unrighteous

àsúvaros, -ov, unable, impossible

$\dot{\alpha} x \alpha \alpha$ apros, -оv, unclean

$\dot{\alpha} \mu \dot{n} v$, adv., truly, verily

$\ddot{\alpha} \pi \alpha \varsigma, \check{\alpha} \pi \alpha \sigma \alpha, \ddot{\alpha} \pi \alpha \nu$, all, altogether

$\dot{\alpha} \pi 0 \lambda \dot{u} \omega$, I release

Bıß入iov, tó, book, a written document

$\theta \varepsilon p \alpha \tau \varepsilon \dot{\omega} \omega$, I heal

$\gamma_{\tau \varepsilon,}$ relative temporal adv. with the indicative, when or $\alpha \nu$, relative temporal adv. with the subj. and indicative, whenever, when

$\pi \tilde{\alpha} \varsigma, \pi \tilde{\alpha} \sigma \alpha, \pi \tilde{\alpha} \nu$, all, every

320. The first aorist active participle of $\lambda \dot{v} \omega$ is $\lambda \dot{\delta} \sigma \alpha \varsigma$

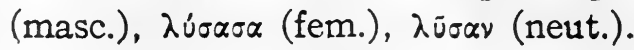


STEM $\lambda u \sigma \alpha \nu \tau-$

\section{Singular}

\begin{tabular}{|c|c|c|c|}
\hline & Masc. & Fem. & Neut. \\
\hline Nom. & $\lambda u ́ \sigma \alpha \varsigma$ & $\lambda \dot{u} \sigma \alpha \sigma \alpha$ & $\lambda \bar{u} \sigma \alpha \nu$ \\
\hline Gen. & 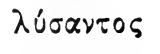 & $\lambda u \sigma \alpha ́ \sigma \eta s$ & 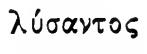 \\
\hline Abl. & 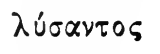 & $\lambda u \sigma \alpha ́ \sigma \eta s$ & 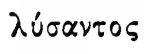 \\
\hline Loc. & 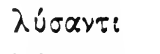 & $\lambda u \sigma \alpha ́ \sigma n$ & $\lambda u ́ \sigma \alpha v \tau \ell$ \\
\hline Ins. & $\lambda u ́ \sigma \alpha v \tau !$ & $\lambda u \sigma \alpha ́ \sigma n$ & $\lambda \dot{\sigma} \sigma \alpha \nu \tau \ell$ \\
\hline Dat. & $\lambda u ́ \sigma \alpha v \tau \ell$ & $\lambda u \sigma \alpha ́ \sigma n$ & $\lambda \dot{\sigma} \sigma \alpha \nu \tau \ell$ \\
\hline Acc. & $\lambda u ́ \sigma \alpha \nu \tau \alpha$ & $\lambda u ́ \sigma \alpha \sigma \alpha \nu$ & $\lambda \tilde{u} \sigma \alpha \nu$ \\
\hline \multicolumn{4}{|c|}{ Plural } \\
\hline Nom. & $\lambda \dot{\sigma} \sigma \alpha \nu \tau \varepsilon \varsigma$ & $\lambda \cup ́ \sigma \alpha \sigma \alpha \iota$ & $\lambda \dot{U} \sigma \alpha \nu \tau \alpha$ \\
\hline Gen. & $\lambda u \sigma \alpha ́ \alpha \tau \omega \nu$ & $\lambda \cup \sigma \alpha \sigma \bar{\omega} \nu$ & $\lambda \cup \sigma \alpha^{\prime} \nu \tau \omega \nu$ \\
\hline Abl. & 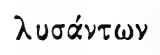 & $\lambda \cup \sigma \alpha \sigma \bar{\omega} \nu$ & $\lambda \cup \sigma \alpha^{\prime} \nu \tau \omega \nu$ \\
\hline Loc. & $\lambda u ́ \sigma \alpha \sigma \ell$ & $\lambda u \sigma \alpha ́ \sigma \alpha ! s$ & $\lambda \dot{v} \sigma \alpha \sigma \iota$ \\
\hline Ins. & $\lambda u ́ \sigma \alpha \sigma \ell$ & $\lambda u \sigma \dot{\alpha} \sigma \alpha \iota \varsigma$ & $\lambda \dot{\sigma \sigma \alpha \sigma \ell}$ \\
\hline Dat. & $\lambda \dot{\sigma} \sigma \alpha \sigma t$ & $\lambda \cup \sigma \alpha ́ \sigma \alpha ! \varsigma$ & $\lambda \dot{U} \sigma \alpha \sigma \ell$ \\
\hline Acc. & $\lambda u ́ \sigma \alpha \nu \tau \alpha \varsigma$ & $\lambda \cup \sigma \alpha ́ \sigma \alpha \varsigma$ & $\lambda \dot{\sigma} \sigma \alpha \nu \tau \alpha$ \\
\hline
\end{tabular}

a. All participles with masc. nom. in $-\alpha \varsigma$ are declined like $\lambda \dot{v} \sigma \alpha \varsigma, \lambda \dot{u} \sigma \alpha \sigma \alpha, \lambda \tilde{u} \sigma \alpha \nu$.

321. Observe: I. The aorist tense suffix $\sigma \alpha$ appears throughout. 2. To the aorist theme is added the participial ending - $-\nu \tau$. 3. The stem is $\lambda \cup \sigma \alpha \nu \tau-$; and (I) to this is added $\varsigma$ to form the nominative masc. sing.; $\nu \tau$ cannot stand before $\varsigma$ and drops out, and $\alpha$ is lengthened; (2) the neuter nom. sing. is the mere stem, $\tau$ being dropped $(234,4) ;(3)$ the fem. nom. sing. $\lambda u \sigma \alpha \sigma \alpha$ is from $\lambda u \sigma \alpha \nu \sigma \alpha$ from $\lambda u \sigma \alpha \nu \tau i \alpha$ (see 234, 3 footnote). 4. The masc. and neuter are declined according to the third declension, and the fem. is declined according to the first declension. 
322. The first aorist middle participle of $\lambda \dot{\omega} \omega$ is

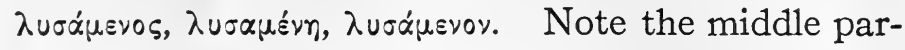
ticiple ending $-\mu \varepsilon \nu \circ \varsigma,-\mu \varepsilon \nu \eta,-\mu \varepsilon v \circ \nu$ is added to the aorist theme $\lambda u \sigma \alpha-$. The first aorist middle participle, like the present and second aorist middle participles, is declined like an adjective of the first and second declensions.

323. Of course the kind of action (punctiliar) and the Aktionsart of the verb-stem apply to the participle.

324. The declension of $\pi \tilde{\alpha} \varsigma, \pi \tilde{\alpha} \sigma \alpha, \pi \tilde{\alpha} \nu$ is:

\section{STEM $\pi \alpha \nu \tau-$}

\section{Singular}

\section{Masc.}

Nom. $\quad \pi \tilde{\alpha} \varsigma$

Gen. $\pi \alpha \nu \tau \delta \varsigma$

Abl. $\pi \alpha v \tau b s$

Loc. $\pi \alpha \nu \tau \ell$

Ins. $\quad \pi \alpha \nu \tau \hat{t}$

Dat. $\pi \alpha v \tau i$

Acc. $\quad \pi \dot{\alpha} v \tau \alpha$
Fem.

$$
\pi \tilde{\alpha} \sigma \alpha
$$

$\pi \dot{\alpha} \sigma \eta s$

$\pi \dot{\alpha} \sigma \eta \operatorname{s}$

$\pi \dot{\alpha} \sigma n$

$\pi \dot{\alpha} \sigma n$

$\pi \dot{\alpha} \sigma n$

$\pi \tilde{\alpha} \sigma \alpha \nu$

\section{Plural}

Nom. $\pi \alpha^{\prime} \nu \tau \varepsilon \varsigma$

Gen. $\quad \pi \dot{\alpha} \nu \tau \omega \nu$

Abl. $\pi \dot{\alpha} \nu \tau \omega \nu$

Loc. $\quad \pi \tilde{\alpha} \sigma$ เ

Ins. $\quad \pi \tilde{\alpha} \sigma \iota$

Dat. $\quad \pi \tilde{\alpha} \sigma \iota$

Acc. $\pi \dot{\alpha} \nu \tau \alpha \varsigma$

$\begin{array}{ll}\pi \bar{\alpha} \sigma \alpha \iota & \pi \dot{\alpha} \nu \tau \alpha \\ \pi \alpha \sigma \tilde{\omega} \nu & \pi \dot{\alpha} \nu \tau \omega: \\ \pi \alpha \sigma \tilde{\omega} \nu & \pi \dot{\alpha} \nu \tau \omega ! \\ \pi \dot{\alpha} \sigma \alpha ! \varsigma & \pi \tilde{\alpha} \sigma l \\ \pi \dot{\alpha} \sigma \alpha ! \varsigma & \pi \tilde{\alpha} \sigma l \\ \pi \dot{\alpha} \sigma \alpha ! \varsigma & \pi \tilde{\alpha} \sigma ! \\ \pi \dot{\alpha} \sigma \alpha \varsigma & \pi \dot{\alpha} \nu \tau \alpha\end{array}$

Neut.

$\pi \tilde{\alpha} \nu$

$\pi \alpha v \tau \delta$ s

Taviós

$\pi \alpha \nu \tau i$

$\pi \alpha \nu \tau i$

$\pi \alpha \nu \tau i$

$\pi \tilde{\alpha} \nu$

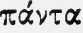


$a$. $\ddot{\alpha} \pi \alpha_{\varsigma}$ is a strengthened form of $\pi \tilde{\alpha} \varsigma$ and except for the accent is declined like $\pi \tilde{\alpha} \varsigma$.

325. I. Note that except for the accent. $\pi \tilde{\alpha} \varsigma$ is declined like the first aorist participle $\lambda \dot{v} \sigma \alpha \varsigma$; and the formation of genders and cases is like $\lambda \dot{\sigma} \sigma \alpha \varsigma$.

2. Observe that the accent in the masculine and neuter singular is that of monosyllables of the third declension (226), while in the plural the accent is on the penult.

326. Examine carefully the following examples of the use of $\pi \tilde{\alpha} \varsigma$ :

I. In the predicate position:
a. $\pi \tilde{\alpha} \sigma \alpha \dot{\eta} \pi \delta \lambda_{l} s, \quad$ all the city
b. $\pi \tilde{\alpha} \sigma \alpha \iota \alpha i \pi \delta \lambda_{\varepsilon} \varsigma$, all cities

2. In the attributive position:

a. $\dot{\eta} \pi \tilde{\alpha} \sigma \alpha \pi \dot{\delta} \lambda_{\text {s }}, \quad$ the city as a whole

b. $\delta \pi \tilde{\alpha} \varsigma$ vouos, the entire law, the whole law

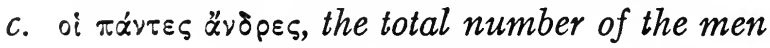

3. With a singular substantive, without the article; $\pi \tilde{\alpha} \sigma \alpha \pi \dot{\delta} \lambda_{\imath \varsigma}$ or $\pi \dot{\delta} \lambda_{1 \varsigma} \pi \tilde{\alpha} \sigma \alpha$, every city

4. $\pi \tilde{\alpha} \varsigma \dot{\delta}$ and the participle is a common construction in the New Testament.

$\pi \tilde{\alpha} \varsigma \dot{\alpha} \alpha \times 0 \dot{\omega} \omega \nu$,

every one hearing, every one who hears

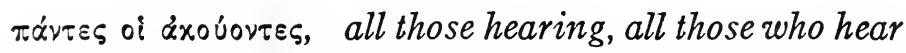

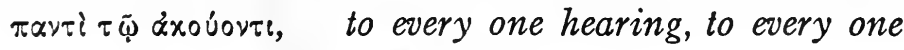
who hears

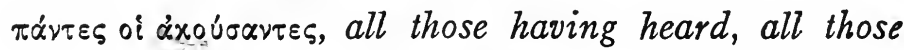
who heard 


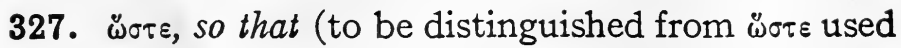
as an inferential conjunction, and so, therefore) is used with the infinitive (twice with the indicative) to express result:

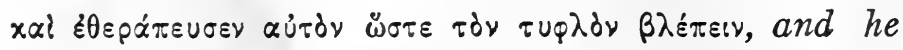
healed him so that the blind man was seeing.

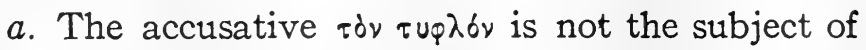
the infinitive $\beta \lambda \varepsilon \dot{\pi \varepsilon \iota \nu}$. The infinitive does not have a subject; it has no personal endings, and is not a finite verb. The acc. limits an idea in content, scope, and direction. The action in $\beta \lambda \varepsilon \pi \varepsilon \varepsilon \nu$ is

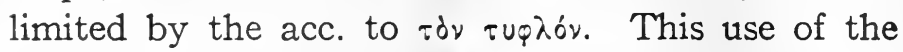
acc. is generally called the acc. of general reference.

328.

EXERCISES

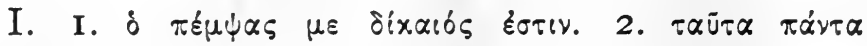

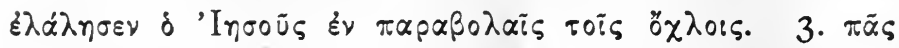

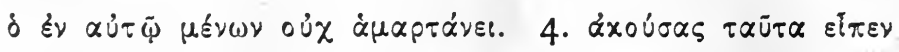

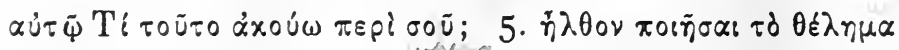

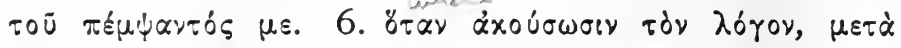

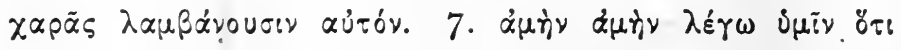

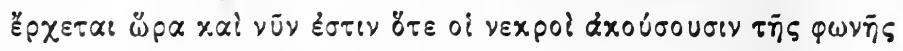

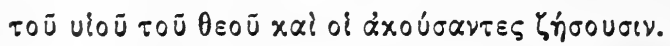

II. I. Having heard this he went away. 2. He healed all the blind so that they marvelled. 3. Every one believing on him comes not into judgment. 4. When you hear his voice, you will believe. 5. Let us hear him who sent him. 


\section{ADJECTIVES OF THE THIRD DECLENSION 133}

\section{LESSON XXXIX}

Adjectives of the Third Declension: Stems in $-\varepsilon \varsigma$.

Irregular Adjectives, $\pi 0 \lambda \cup u_{\varsigma}$ and $\mu \varepsilon^{\prime} \gamma \alpha \varsigma$

329.

$\dot{\alpha} \lambda \eta \theta \dot{\eta} \varsigma,-\hat{\varepsilon} \varsigma$, true

$\dot{\alpha} \sigma \theta \varepsilon v \dot{\eta} \varsigma,-\varepsilon \xi$, weak, sick

$\gamma \dot{\alpha} \mu \circ \varsigma, \delta$, marriage

$\mu \varepsilon \hat{\varepsilon} \gamma \alpha \varsigma, \mu \varepsilon \gamma \alpha^{\prime} \lambda \eta, \mu \varepsilon^{\prime} \gamma \alpha$, great

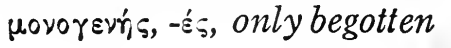

\section{VOCABULARY}

$\pi \circ \lambda \dot{\varsigma} \varsigma, \pi 0 \lambda \lambda \dot{\eta}, \pi \circ \lambda \dot{u}, m u c h$; many

$\pi$ poosuxín, $\dot{\eta}$, prayer i $\pi \dot{\alpha} \gamma \omega$, I go away, depart

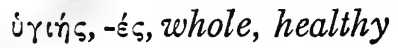

330. The declension of $\dot{\alpha} \lambda \eta \theta \dot{\eta} \dot{s}$ is:

STEM $\alpha \lambda \eta \eta \theta \sigma-$

\section{Singular}

\section{Masc. and Fem.}

Nom. $\dot{\lambda} \lambda \eta \dot{\eta} \dot{n}$

Gen. $\quad \alpha \lambda \eta \theta_{0} \tilde{u} s$

Abl. $\alpha \lambda \eta \theta 00 \tilde{s}$

Loc. $\quad \alpha \lambda r \theta \theta \bar{\imath}$

Ins. $\quad \dot{\alpha} \lambda \eta \theta \varepsilon i$

Dat. $\quad \alpha \lambda \eta \theta \varepsilon i$

Acc.

$\dot{\alpha} \lambda \eta \theta \tilde{\eta}$

\section{Plural}

Masc. and Fem.

Nom. $\quad \alpha \lambda \eta \theta \varepsilon i \varsigma$

Gen. $\quad \dot{\lambda} \lambda \eta \dot{\theta} \theta \tilde{\omega}$

Abl. $\quad \dot{\lambda} \lambda r \theta \tilde{\omega} \nu$

Loc. $\quad \dot{\lambda} \eta \eta \theta \varepsilon$

Ins. $\quad \alpha \lambda \eta, \theta \varepsilon \dot{\varepsilon}$

Dat. $\quad \dot{\alpha} \eta \eta \theta \varepsilon \sigma \varepsilon$

Acc.
Neut.

$\dot{\alpha} \lambda \eta \theta \theta \dot{\varepsilon} \varsigma$

$\dot{\alpha} \lambda \eta \theta 0 \tilde{\cup} s$

$\dot{\alpha} \lambda \eta \theta 0 \tilde{\Delta} s$

$\alpha \lambda \eta \theta \varepsilon \tilde{\varepsilon}$

$\dot{\alpha} \lambda \eta \theta \theta \tilde{\varepsilon}$

$\dot{\alpha} \lambda \eta \theta \varepsilon \tilde{\varepsilon}$

$\dot{\alpha} \lambda \eta \theta \hat{\varepsilon} \varsigma$

Neut.

$\dot{\alpha} \lambda \eta \theta \tilde{n}$

$\dot{\alpha} \lambda \eta \theta \tilde{\omega} \nu$

$\dot{\alpha} \lambda \eta \theta \tilde{\omega} \nu$

$\alpha \lambda \eta \eta \dot{\varepsilon} \sigma 6$

$\dot{\alpha} \lambda \eta \theta \dot{\varepsilon} \sigma$

$\dot{\alpha} \lambda \eta \theta \dot{\varepsilon} \sigma$

$\dot{\alpha} \lambda \eta_{2} \theta \tilde{n}$ 
There are about sixty adjectives in the New Testament declined like $\dot{\alpha} \lambda \eta \dot{\theta} \theta \dot{\eta}$.

Compare the declension of $\alpha \lambda \eta \theta \hat{\theta} s$ with $\gamma \varepsilon$ vos (290).

331. In the declension of $\alpha \lambda \eta \theta \dot{\eta} \varsigma$ observe: $\mathbf{I}$. The neuter nom. and acc. sing. is the mere stem. 2. The masc. nom. sing. is the stem with the stem vowel lengthened. 3. In the other cases $\sigma$ of the stem drops out and the concurrent vowels contract. The gen. and abl. sing. $\alpha \lambda \eta \theta 0 \tilde{\nu} \varsigma$ is from $\alpha \lambda \eta \theta \varepsilon \dot{\varepsilon} \sigma \varsigma(\varepsilon+0=0 u)$; the loc., ins., and dat. sing. $\alpha \lambda \eta \theta=\tilde{\imath}$ is from $\alpha \lambda \eta \theta \varepsilon \sigma$ $(\varepsilon+\imath=\varepsilon \iota)$; the masc. acc. sing. $\alpha \lambda \eta \theta \tilde{\eta}$ is from $\alpha \lambda \eta \theta \dot{\varepsilon} \sigma \alpha$ $(\varepsilon+\alpha=\eta)$; the masc. nom. plur. $\alpha \lambda \eta \theta \theta i s$ is from $\alpha \lambda \eta \theta \varepsilon \varepsilon \varepsilon s(\varepsilon+\varepsilon=\varepsilon \iota)$; the neut. nom. and acc. plur. $\dot{\alpha} \lambda \eta \theta \tilde{\eta}$ is from $\alpha \lambda \eta \theta \dot{\varepsilon} \sigma \alpha(\varepsilon+\alpha=\eta)$; the gen. plural $\alpha \lambda \eta \theta \tilde{\omega} \nu$ is from $\alpha \lambda \gamma \theta \varepsilon \delta \omega \nu(\varepsilon+\omega=\omega)$; the masc. and fem. acc. plur. $\alpha \lambda r_{1} \theta$ sis is like the nom. (probably borrowed from the nom.). 4. The masc. and fem. forms are alike. (This is the first adjective given thus far, the feminine of which is declined in the third declension.) 5. The gen., abl., loc., ins. and dat. in all three genders are alike.

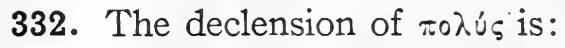

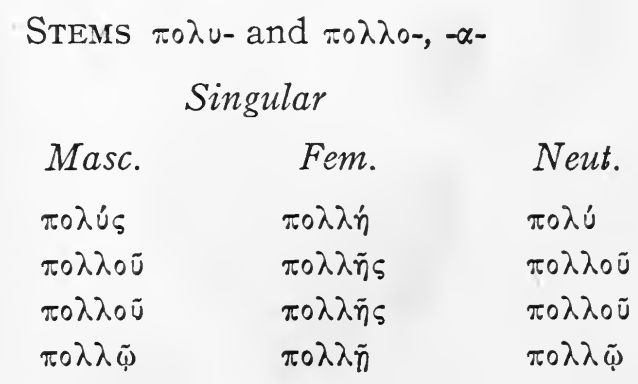

Nom. $\quad \pi 0 \lambda$ ús

Gen. $\quad \pi 0 \lambda \lambda \lambda_{0} \tilde{0}$

Abl. $\pi \circ \lambda \lambda \circ \tilde{U}$

Loc. $\quad \pi 0 \lambda \lambda \tilde{\varphi}$

$\pi \circ \lambda \lambda \tilde{n}$

$\pi \circ \lambda \lambda \tilde{\varphi}$ 


\section{IRREGULAR ADJECTIVES}

\begin{tabular}{|c|c|c|c|}
\hline & Masc. & Fem. & Neut. \\
\hline Ins. & $\pi 0 \lambda \lambda \bar{\varphi}$ & $\pi 0 \lambda \lambda \bar{n}$ & $\pi 0 \lambda \lambda \tilde{\varphi}$ \\
\hline Dat. & $\pi 0 \lambda \lambda \bar{\varphi}$ & $\pi 0 \lambda \lambda \bar{n}$ & $\pi 0 \lambda \lambda \tilde{\varphi}$ \\
\hline Acc. & $\pi 0 \lambda u ́ v$ & $\pi 0 \lambda \lambda \dot{\eta} \nu$ & $\pi 0 \lambda \dot{0}$ \\
\hline \multicolumn{4}{|c|}{ Plural } \\
\hline Nom. & $\pi 0 \lambda \lambda 06$ & $\pi 0 \lambda \lambda \alpha \ell$ & $\pi 0 \lambda \lambda \alpha \dot{\alpha}$ \\
\hline Gen. & $\pi 0 \lambda \lambda \bar{\omega} \nu$ & $\pi 0 \lambda \lambda \lambda \bar{\omega} \nu$ & $\pi 0 \lambda \lambda \bar{\omega} \nu$ \\
\hline Abl. & $\pi 0 \lambda \lambda \lambda \tilde{\omega} \nu$ & $\pi 0 \lambda \lambda \lambda \bar{\omega} \nu$ & $\pi 0 \lambda \lambda \bar{\omega} \nu$ \\
\hline Loc. & $\pi 0 \lambda \lambda 0 i s$ & $\pi 0 \lambda \lambda \alpha i s$ & $\pi 0 \lambda \lambda \lambda_{0 i s}$ \\
\hline Ins. & $\pi 0 \lambda \lambda \circ \pi_{\varsigma} \varsigma$ & $\pi 0 \lambda \lambda \alpha \tilde{i} \varsigma$ & $\pi 0 \lambda \lambda_{0} \pi i s$ \\
\hline Dat. & $\pi 0 \lambda \lambda 0 i s$ & $\pi 0 \lambda \lambda \alpha \tilde{i} \varsigma$ & $\pi 0 \lambda \lambda \lambda_{0 i \zeta}$ \\
\hline Acc. & $\pi 0 \lambda \lambda \circ u ́ \varsigma$ & $\pi 0 \lambda \lambda \alpha^{\prime} \varsigma$ & $\pi 0 \lambda \lambda \alpha^{\prime}$ \\
\hline
\end{tabular}

Observe: I. The masc. and neut. nom. and acc. sing. are made on the stem $\pi 0 \lambda_{u}$. 2 . All the other cases (masc. fem. and neut.) are made on the stem $\pi 0 \lambda \lambda_{0}$ - (fem. end. $-\eta$ ) and declined according to the first and second declensions.

333. The declension of $\mu \varepsilon \gamma^{\prime} \alpha \varsigma$ is:

STEMS $\mu \varepsilon \gamma \alpha-$ and $\mu \varepsilon \gamma \alpha \lambda_{0-},-\alpha-$

\section{Singular}

\section{Masc. Fem.}

Nom. $\mu \varepsilon^{\prime} \gamma \alpha s$

Gen. $\quad \mu \varepsilon \gamma \alpha \dot{\alpha} \lambda_{0 u}$

Abl. $\mu \varepsilon \gamma \dot{\alpha} \lambda \circ 0$

Loc. $\quad \mu \varepsilon \gamma \dot{\alpha} \lambda \omega$

Ins. $\quad \mu \varepsilon \gamma \dot{\alpha} \lambda \omega$

Dat. $\quad \mu \varepsilon \gamma \alpha \dot{\alpha} \lambda \omega$

Acc. $\mu \varepsilon^{\prime} \gamma \alpha \nu$

\begin{tabular}{|c|c|}
\hline$\mu \varepsilon \gamma \alpha^{\prime} \lambda \eta$ & $\mu \varepsilon^{\prime} \gamma \alpha$ \\
\hline$\mu \varepsilon \gamma \alpha^{\prime} \lambda \eta \zeta$ & $\mu \varepsilon \gamma \alpha^{\prime} \lambda \circ u$ \\
\hline$\mu \varepsilon \gamma \alpha^{\prime} \lambda \eta \varsigma$ & $\mu \varepsilon \gamma \alpha^{\prime} \lambda \circ v$ \\
\hline$\mu \varepsilon \gamma \alpha^{\prime} \lambda n$ & $\mu \varepsilon \gamma \alpha^{\prime} \lambda \varphi$ \\
\hline$\mu \varepsilon \gamma \alpha \dot{\alpha} \lambda n$ & $\mu \varepsilon \gamma \alpha^{\prime} \lambda \varphi$ \\
\hline$\mu \varepsilon \gamma \alpha^{\prime} \lambda_{n}$ & $\mu \varepsilon \gamma \alpha^{\prime} \lambda \omega$ \\
\hline$\mu \varepsilon \gamma \alpha^{\prime} \lambda \eta \nu$ & $\mu \varepsilon^{\prime} \gamma(x$ \\
\hline
\end{tabular}

Neut.

$\mu \varepsilon \gamma \alpha$

$\mu \varepsilon \gamma \alpha \lambda_{00}$

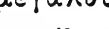

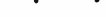

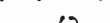

$\mu \varepsilon \hat{\gamma u}$ 


\section{Plural}

\begin{tabular}{|c|c|c|c|}
\hline & Masc. & Fem. & Neut. \\
\hline Nom. & $\mu \varepsilon \gamma \alpha^{\prime} \lambda \circ 6$ & $\mu \varepsilon \gamma \alpha^{\prime} \lambda \alpha_{\ell}$ & $\mu \varepsilon \gamma \alpha^{\prime} \lambda \alpha$ \\
\hline Gen. & $\mu \varepsilon \gamma \alpha^{\prime} \lambda \omega \nu$ & $\mu \varepsilon \gamma \alpha^{\prime} \lambda \omega \nu$ & $\mu \varepsilon \gamma \alpha^{\prime} \lambda \omega \nu$ \\
\hline Abl. & $\mu \varepsilon \gamma \alpha^{\prime} \lambda \omega \nu$ & $\mu \varepsilon \gamma \alpha^{\prime} \lambda \omega \nu$ & $\mu \varepsilon \gamma \alpha^{\prime} \lambda \omega \nu$ \\
\hline Loc. & $\mu \varepsilon \gamma \alpha \dot{\lambda}$ ols & $\mu \varepsilon \gamma \alpha^{\prime} \lambda \alpha t \varsigma$ & $\mu \varepsilon \gamma \alpha \dot{\lambda} 0: \varsigma$ \\
\hline Ins. & $\mu \varepsilon \gamma \alpha \dot{\lambda} 0: \varsigma$ & $\mu \varepsilon \gamma \alpha^{\prime} \lambda \alpha ! \varsigma$ & $\mu \varepsilon \gamma \alpha^{\prime} \lambda$ ol $\varsigma$ \\
\hline Dat. & $\mu \varepsilon \gamma \alpha^{\prime} \lambda$ ols & $\mu \varepsilon \gamma \alpha^{\prime} \lambda \alpha \iota \varsigma$ & $\mu \varepsilon \gamma \alpha^{\prime} \lambda O \iota \varsigma$ \\
\hline Acc. & $\mu \varepsilon \gamma \alpha^{\prime} \lambda \circ \cup \varsigma$ & $\mu \varepsilon \gamma \alpha^{\prime} \lambda \alpha \varsigma$ & $\mu \varepsilon \gamma \alpha^{\prime} \lambda \alpha$ \\
\hline
\end{tabular}

Observe: I. The masc. and neut. nom. and acc. sing. are made on the short stem $\mu \varepsilon \gamma \alpha-$.

2. All the other cases (masc., fem., and neut.) are made on the long stem $\mu \varepsilon \gamma \alpha \lambda_{0}-$ (fem. end. $-\eta$ ) and are declined like adjectives of the first and second declensions.

334. Study carefully the following examples of indirect discourse:

I. Indirect assertions. I. After $\gamma \tau$ (that):

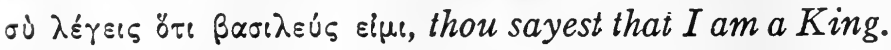

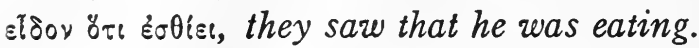

2. With the infinitive:

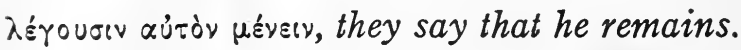

3. With the participle:

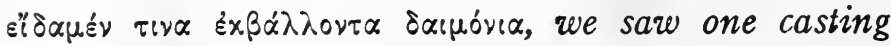
out demons.

II. Indirect questions:

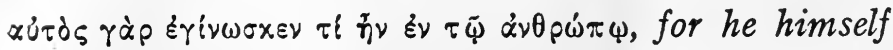
knew what was in man.

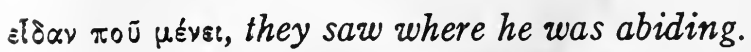

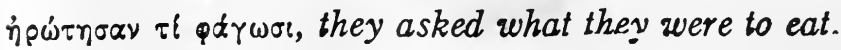


III. Indirect commands.

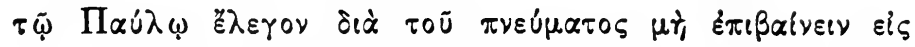
'Ispoob $u \mu \alpha$, they said to Paul through the spirit that he should not go up to Jerusalem (literally, not to go up to Jerusalem).

335. Observe:

I. Indirect assertions are expressed (I) by ${ }^{\circ} \tau \iota$ and the indicative; (2) by the infinitive; (3) by the participle.

2. The same introductory words are generally used in indirect questions as those which are found in direct questions.

3. The infinitive is frequently used in indirect commands.

$a$. Sometimes $i_{\nu \alpha}$ and a finite mode is used. ${ }^{1}$

4. The tense generally remains unchanged in the Greek indirect discourse.

a. Sometimes there is a change, as in the first example under II; the imperfect $\eta^{\gamma} \nu$ seems to represent a present in the direct.

5. The mode generally remains unchanged in the Greek indirect discourse. The subjunctive mode ( $\phi \alpha \dot{\gamma} \gamma \sigma \iota)$ in the third example under II was in the direct.

6. The person of the verb is or is not changed according to the circumstances.

In the third example under II the third person was first or second in the direct.

${ }^{1}$ Also used as object-clause after verbs of striving, beseeching, etc. 
336.

EXERCISES

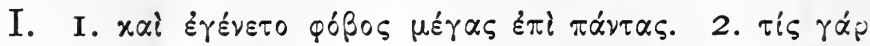

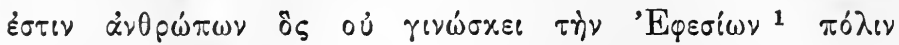

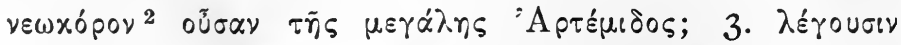

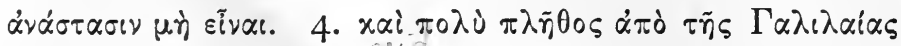

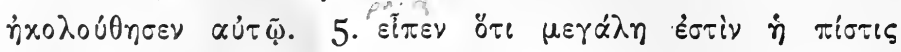

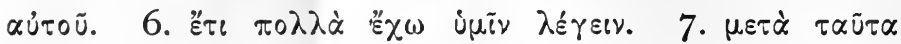

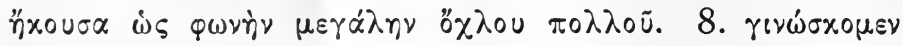
ö $\tau \iota \dot{\alpha} \lambda \eta \theta \dot{\eta} \varsigma \varepsilon \tilde{l}$.

II. I. They did not know what they would see. 2. They know that there shall be great tribulation. 3. Many saw the Lord coming and said that he was the Saviour of the world. 4. This one is the only begotten son.

\section{LESSON XI}

First Aorist Passive Indicative and Subjunctive. Future Passive Indicative

337. VOCABULARY

$\dot{\alpha} \nu \alpha \gamma e v \omega \dot{\sigma} \times \omega$, I read $\lambda \cup \pi \varepsilon ́ \omega, I$ grieve

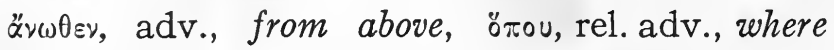
again

aüprov, adv., tomorrow $\pi \dot{0} \theta \varepsilon v$, interrogative adv., whence

ĖrYús, adv., near $\sigma \alpha \lambda \varepsilon \dot{\omega} \omega, I$ shake

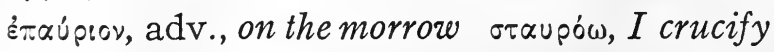

338. The stem of first aorist passive is formed by adding $\theta \varepsilon$ directly to the verb-stem. Thus $\lambda u \theta \varepsilon-$ is the first aorist stem of $\lambda \dot{u} \omega$.

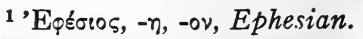

2 vewxbpos, $\dot{\delta}$ or $\dot{\eta}$, temple-keeper
} 


\section{FIRST AORIST PASSIVE}

339. The first aorist passive indicative of $\lambda \dot{\omega} \omega$ is: Singular

Plural

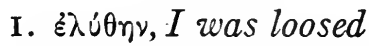

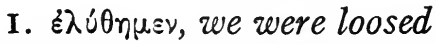

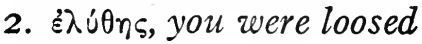

2. Ėं $\dot{\theta} \theta \tau \varepsilon$, you were loosed

3. ह่入ún, he was loosed

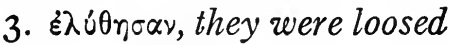

First aorist passive infinitive- $\lambda u \theta \tilde{\eta} v \alpha$, to be loosed

Observe: I. The vowel $\varepsilon$ of the aorist passive tense suffix $\theta \varepsilon$ is lengthened to $\eta(\theta \eta)$ throughout the indicative, and in the infinitive. 2. The personal endings of the aorist passive indicative are the secondary active personal endings (66). 3. The endings are added directly to the aorist passive stem. 4. In the indicative there is an augment as in the aorist active ind. ( 182,3 and 296,3 ), and is formed on the same principles as in the imperfect (70). 5. The aorist passive infinitive ending is $-\nu \alpha$; the accent of the aorist passive infinitive is always on the penult.

340. The aorist passive subjunctive of $\lambda \dot{v} \omega$ is:
Singular
Plural
I. $\lambda \cup \theta \tilde{\omega}$
I. $\lambda \cup \theta \tilde{\omega} \mu \varepsilon \nu$
2. $\lambda \cup \theta \tilde{n} s$
2. $\lambda u \theta \tilde{\eta} \tau \varepsilon$
3. $\lambda \cup \theta \tilde{n}$
3. $\lambda u \theta \tilde{\omega} \sigma$

Observe: I. The subjunctive has the primary personal endings. 2. The subjunctive mode sign $\omega / \eta$ contracts with $\varepsilon$ of the passive suffix. 3. The circumflex accent is written over the contracted syllable. 4. The subjunctive does not have an augment.

341. Review the "kind of action" (punctiliar) of the aorist tense and Aktionsart of the verb-stem, 
I78-I80, 305-307. These of course apply to the zorist passive as well as to active and middle.

342. For the meaning of the passive voice see $5 \mathrm{I}$. Some defective ( $\left.1_{52}\right)$ verbs have no aorist middle, but passive form; but not the passive meaning: the meaning is either middle or active (or intransitive):

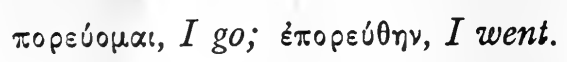

Some verbs have both aorist middle and passive forms, as $\dot{\alpha} \pi \varepsilon x \rho เ v \dot{\alpha} \mu \eta \eta, \dot{\alpha} \pi \varepsilon x \rho i \theta \eta \eta$.

343. Verbs with stems ending in a short vowel $(\alpha, \varepsilon, 0)$ generally lengthen ( $\breve{\alpha}$ to $\bar{\alpha}$ after $\varepsilon$, l, or $\rho$ ) the vowel of the stem before the tense suffix $\theta \varepsilon$ in formation of the aorist passive. Thus:

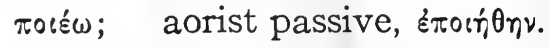

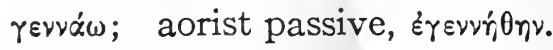

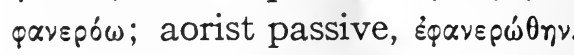

$a$. A few verbs like $\tau \varepsilon \lambda \varepsilon \hat{\varepsilon}$ do not lengthen $\varepsilon$; but have $\sigma$ before $\theta \varepsilon$, as $\varepsilon \tau \varepsilon \lambda \varepsilon \dot{\sigma} \theta \eta \eta$.

344. The future passive is made upon the aorist passive stem. Thus $\lambda u \theta$ nooucl, I shall be loosed, is the aorist passive stem $\lambda u \theta \eta+\sigma o$ (the future tense suffix and thematic vowel) + $\alpha_{i}$ (the primary middle ending). The future passive of $\lambda \dot{\omega} \omega$ is:

\section{Singular}

Plural

I. $\lambda \cup \theta \dot{n} \sigma \circ \mu \alpha$, $I$ shall be loosed I. $\lambda \cup \theta \eta \sigma \delta \mu \varepsilon \theta \alpha$, we shall be

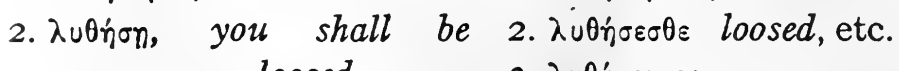
loosed

3. $\lambda u \theta \dot{n} \sigma \varepsilon \tau \alpha$ he, she or it shall be loosed 
Observe that the future passive is conjugated like the future middle except that the stem of the passive is $\lambda u \theta \eta \sigma-$, whereas the stem of the middle is $\lambda \cup \sigma-$.

345. In some verbs (having no first aorist passive) there is found a second aorist passive with suffix $-\varepsilon(-\eta)$ added directly to the verb-stem. The conjugation is like that of the first aorist passive, except there is no $\theta$.

Thus: the second aorist passive of $\gamma \rho \alpha \dot{\alpha} \omega$ is

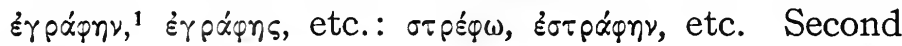
aorist passive infinitive Yeapñ $v$.

The second future passive is built on the second aorist stem. The second future passive of $\varphi \alpha i v \omega$

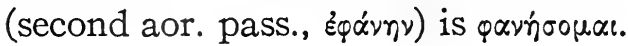

\section{6.}

\section{EXERCISES}

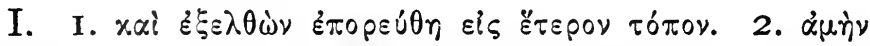

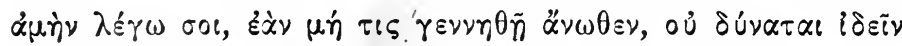

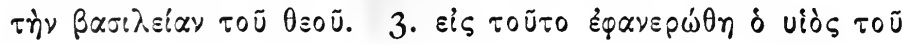

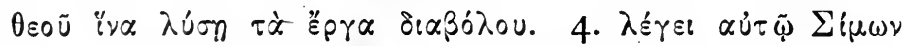

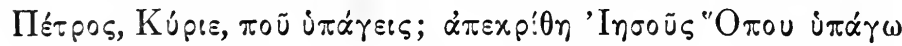

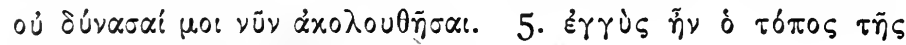

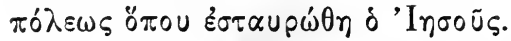

II. I. If he be made manifest, we shall be like

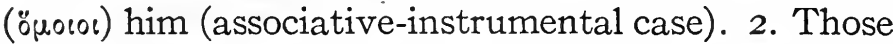
who believed were begotten of ( $\dot{\varepsilon} x)$ God. 3. It was written in order that the Scripture might be fulfilled. 4. The powers of the heavens shall be shaken.

${ }_{1}$ The second aorist passive is really an active form that came to have a passive meaning. 


\section{LESSON XLI}

\section{Aorist Passive (Continued)}

347.

VOCABULARY

$\dot{\alpha} \lambda \eta \dot{\theta} \omega \varsigma$, adv., truly, surely $\quad x \alpha \lambda \bar{\omega} \varsigma$, adv., finely, well

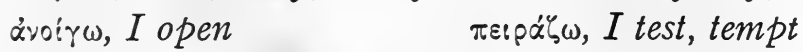

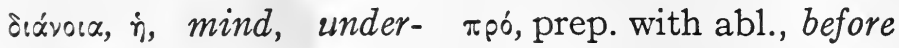
standing $\dot{\varepsilon} \pi \imath \theta$ u’ $\boldsymbol{i}, \dot{\eta}$, desire $\tau \varepsilon \lambda \varepsilon \iota^{\circ} \omega$, I end, complete, fulfill

348. The formation of the first aorist and future passive given in Lesson $\mathrm{XL}$ is typical of all verbs with stems ending in a vowel.

349. Verbs with stems ending in a mute (2I $7-2 \mathrm{I} 8)$ suffer euphonic changes in the mute before the passive suffix $\theta \varepsilon$.

I. A labial mute, $\pi \beta \varphi$, before $\theta$ (of the suffix) becomes $\varphi$. $\pi \dot{\varepsilon} \mu \pi \omega$, stem $\pi \varepsilon \mu \pi-$, aorist passive $\dot{\varepsilon} \pi \dot{\varepsilon} \mu \varphi \theta \eta \eta$.

2. A palatal mute, $x \gamma \chi$, before $\theta$, becomes $\chi$. $\ddot{\alpha} \gamma \omega$, stem $\dot{\alpha} \gamma-$, aorist passive $-\ddot{\eta} \chi \theta \dot{\theta}, \nu$.

3. A lingual mute, $\tau \delta \theta$, before $\theta$ becomes $\sigma$. $\pi \varepsilon i \theta \omega$, stem $\pi \varepsilon \imath \theta$-, aorist passive $\varepsilon \pi \varepsilon i \sigma \theta \eta \nu$.

$\checkmark$ These changes may be represented to the eye in tabular form, thus:

$$
\begin{aligned}
& \pi, \beta, \varphi \text { before } \theta=\varphi \theta . \\
& x, \gamma, \chi \text { before } \theta=\chi^{\theta} . \\
& \tau, \delta, \theta \text {, before } \theta=\sigma \theta .
\end{aligned}
$$

After these changes are made the conjugation follows the form of $\varepsilon \lambda \lambda^{\prime} \theta r_{i} y$. Thus $\lambda \varepsilon i \pi \omega$ :

Aorist passive indicative $\dot{\varepsilon} \lambda \varepsilon i \varphi \theta \eta \eta$, etc.

Aorist passive subjunctive $\lambda \operatorname{se\varphi \theta } \theta \bar{\omega}$, etc.

Aorist passive infinitive $\lambda \varepsilon \iota \varphi \theta \tilde{\eta} \nu \alpha \iota$

Future passive indicative $\lambda \varepsilon \iota \varphi \theta \dot{\eta} \sigma 0 \mu \alpha \ell$, etc. 
350. The stem of the aorist passive participle is made on the aorist passive stem with the participial ending $-\nu \tau$. The stem of the aorist passive participle of $\lambda \dot{u} \omega$ is $\lambda \dot{u} \theta \varepsilon v i-$.

The declension of the aorist passive participle of $\lambda u ́ \omega$ is:

\begin{tabular}{|c|c|c|c|}
\hline \multicolumn{4}{|c|}{ Singular } \\
\hline & Masc. & Fem. & Neut. \\
\hline Nom. & $\lambda u \theta \varepsilon i s$ & $\lambda \cup \theta \varepsilon \tilde{i} \sigma \alpha$ & $\lambda u \theta \dot{\varepsilon v}$ \\
\hline Gen. & 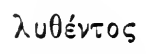 & $\lambda u \theta$ sions & 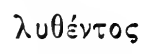 \\
\hline Abl. & 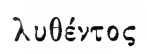 & $\lambda u \theta$ sions & 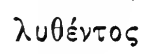 \\
\hline Loc. & 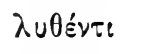 & $\lambda u \theta$ zion & $\lambda u \theta \varepsilon ́ v \tau \iota$ \\
\hline Ins. & 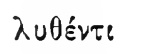 & $\lambda u \theta \varepsilon i \sigma n$ & 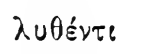 \\
\hline Dat. & $\lambda u \theta \varepsilon ́ v \tau \ell$ & $\lambda u \theta \varepsilon i \sigma n$ & $\lambda u \theta \varepsilon ́ v \tau t$ \\
\hline Acc. & $\lambda u \theta \varepsilon ́ v \tau \alpha$ & $\lambda u \theta \varepsilon \tilde{i} \sigma \alpha \nu$ & $\lambda u \theta \varepsilon^{\prime} \nu$ \\
\hline
\end{tabular}

\section{Plural}

\begin{tabular}{|c|c|c|c|}
\hline Nom. & 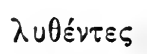 & $\lambda \cup \theta \varepsilon \tilde{\imath} \sigma \alpha \ell$ & 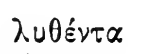 \\
\hline Gen. & $\lambda u \theta \varepsilon ́ v \tau \omega \nu$ & $\lambda \cup \theta \varepsilon เ \sigma \tilde{\omega} \nu$ & 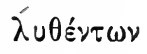 \\
\hline Abl. & $\lambda \cup \theta \varepsilon \dot{\varepsilon} \nu \tau \omega \nu$ & $\lambda \cup \theta \varepsilon \iota \sigma \tilde{\omega} \nu$ & $\lambda \cup \theta \varepsilon ́ v \tau \omega \nu$ \\
\hline Loc. & $\lambda \cup \theta \varepsilon i \sigma \iota$ & $\lambda \cup \theta \varepsilon i \sigma \alpha t \varsigma$ & $\lambda \cup \theta \approx i \sigma t$ \\
\hline Ins. & $\lambda u \theta \varepsilon \tilde{i} \sigma t$ & $\lambda u \theta \varepsilon i \sigma \alpha i \varsigma$ & $\lambda u \theta \varepsilon i \sigma \epsilon$ \\
\hline Dat. & $\lambda \cup \theta \varepsilon i \sigma \sigma$ & $\lambda \cup \theta \varepsilon i \sigma \alpha ! \varsigma$ & $\lambda \cup \theta \varepsilon \tilde{\sigma} \sigma \mathrm{t}$ \\
\hline Acc. & $\lambda u \theta \varepsilon \dot{v} \tau \alpha \alpha \varsigma$ & $\lambda \cup \theta \varepsilon i \sigma \alpha \varsigma$ & 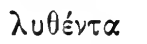 \\
\hline
\end{tabular}

Observe: I. The masc. nom. sing. is formed by adding $-\varsigma$ to the stem $\lambda u \theta \varepsilon v \tau=\lambda u \theta \varepsilon \nu \tau \varsigma ; \nu \tau$ cannot stand before $\varsigma$ and drops out; the $\varepsilon$ is lengthened (compensatory) to $\varepsilon$. 2. The fem. nom. sing. is formed from $\lambda u \theta \varepsilon v \tau \iota \alpha=\lambda u \theta \varepsilon \nu \sigma \alpha=\lambda u \theta \varepsilon เ \sigma \alpha$; $\varepsilon$ is lengthened (compensatory) to $\varepsilon \iota$; see $32 \mathrm{I}, 3$ (3); 234,3 footnote. 3. The neut. nom. (and acc.) sing. is the 


\section{BEGINNER'S GREEK GRAMMAR}

mere stem, without $\tau($ see 234,3$)$. 4. For the forms of the masc. and neut. loc., ins., and dat. plural see 229, 2, and lengthening of $\varepsilon$ to $\varepsilon \iota$ see above. 5. Note the position of the accent on the aorist passive participle in comparison with the other participles.

351. The aorist passive participle of

$$
\begin{aligned}
& \pi \varepsilon \dot{\varepsilon} \mu \omega \omega \text { is } \pi \varepsilon \mu \varphi \theta \varepsilon i \varsigma,-\theta \varepsilon i \sigma \alpha,-\theta \varepsilon \dot{\varepsilon} \text {. } \\
& \ddot{\alpha} \gamma \omega \text { is } \dot{\alpha} \gamma \theta \text { si } \zeta,-\theta \varepsilon i \sigma \alpha,-\theta \dot{\varepsilon} v \text {. } \\
& \pi \varepsilon i \theta \omega \text { is } \pi \varepsilon เ \sigma \theta \varepsilon i \varsigma,-\theta \varepsilon \tilde{\tau} \sigma \alpha,-\theta \varepsilon \dot{v} \text {. } \\
& \gamma \varepsilon \nu \nu \alpha \dot{\alpha} \omega \text { is } \gamma \varepsilon \nu \nu \eta \theta \varepsilon i \zeta,-\theta \varepsilon i \sigma \alpha,-\theta \varepsilon \dot{\varepsilon} \text {. }
\end{aligned}
$$

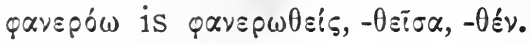

The second aorist passive participle of

$$
\begin{aligned}
& \gamma \rho \alpha \dot{p} \omega \text { is } \gamma p \alpha p \varepsilon i \varsigma,-\varepsilon i \sigma \alpha,-\varepsilon \dot{\nu} \text {. } \\
& \varphi \alpha i v \omega \text { is } \varphi \alpha \nu \varepsilon i \xi,-\varepsilon i \sigma \alpha,-\varepsilon \dot{\gamma} \text {. }
\end{aligned}
$$

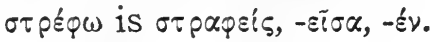

352. The aorist passive participle is used in all the participial constructions that have been studied.

353. It cannot certainly be told beforehand what form of the aorist passive a verb will have. The aorist passive stem must be known.

I. Some of the second aorist passives found in the New Testament (besides those already given) are:

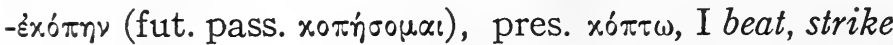
Exxpúßnv, $\varepsilon \dot{\sigma} \sigma \alpha \dot{\alpha} \rho \eta \nu$, pres. xpú $\pi \tau \omega, I$ hide $(\dot{\alpha} \pi) \dot{\varepsilon} \sigma \tau \alpha^{\prime} \lambda \eta \nu$, pres. $\sigma \pi \varepsilon i \rho \omega, I$ sow

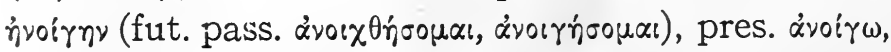
I open 
2. Some verbs have apparently irregular forms in the first aorist and future passive:

\begin{tabular}{|c|c|c|}
\hline Present & First aorist passive & Future passive \\
\hline$\dot{\alpha} x \circ \cup \dot{\omega} \omega$ & $\eta \dot{\eta x} 0 \dot{\sigma} \sigma \theta \eta \nu$ & $\dot{\alpha} \times \circ \cup v \theta \dot{n} \sigma \circ \mu \alpha l$ \\
\hline$\beta \alpha^{\prime} \lambda \lambda \omega$ & $\varepsilon \beta \lambda \dot{\eta} \theta \eta \nu$ & $\beta \lambda_{\eta} \theta \dot{n} \sigma o \mu \alpha \ell$ \\
\hline$\gamma \Leftarrow \nu \omega \dot{\sigma} \sigma \omega$ & $\varepsilon \gamma \gamma \nu \dot{\omega} \sigma \theta \eta \nu$ & $\gamma \nu \omega \sigma \theta \dot{n} \sigma o \mu \alpha t$ \\
\hline Ė $\gamma \varepsilon i \rho \omega$ & $\eta \dot{\gamma \varepsilon} p \theta \eta \nu$ & $\varepsilon \gamma \varepsilon \rho \theta \dot{\eta} \sigma o \mu \alpha \ell$ \\
\hline$x \alpha \lambda \varepsilon^{\prime} \omega$ & $\varepsilon x \lambda \dot{r} \theta \eta \eta$ & $x \lambda \eta \theta \dot{\eta} \sigma o \mu \alpha \varepsilon$ \\
\hline$\lambda \alpha \mu \beta \alpha \dot{\alpha} \nu \omega$ & $\varepsilon \lambda \eta^{\prime} \mu \varphi \theta \eta \nu$ & $-\lambda \eta \mu \varphi \theta \dot{n} \sigma o \mu \alpha_{l}$ \\
\hline Stem ò $\pi-$ & $\dddot{\varphi \theta \eta \nu \nu}$ & 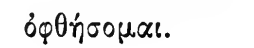 \\
\hline
\end{tabular}

(Presents used, $\beta \lambda \varepsilon ́ \pi \omega$,

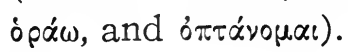

354.

\section{EXERCISES}

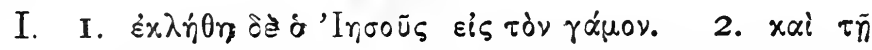

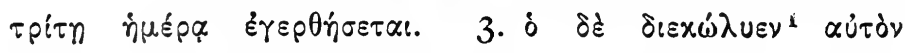

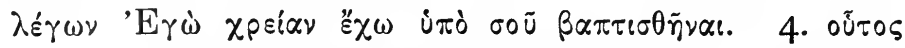

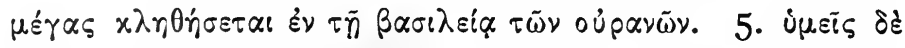

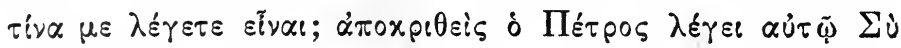

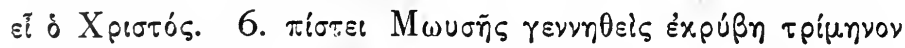

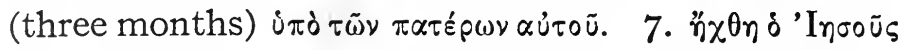

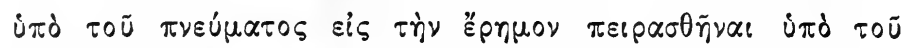

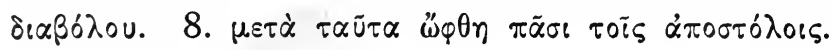

II. I. The prince of this world will be cast out. 2. Let us be led by the spirit. 3. If I touch him, I shall be saved. 4. He who was begotten of God will keep you. 5. If we be raised from the dead, they shall be raised.

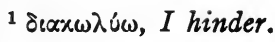




\section{LESSON XIII}

Some Irregular Substantives of the Third Declension.

Some Uses of the Infinitive

355.

VOCABULARY

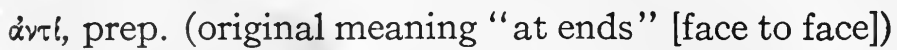
with the gen., opposite, against; instead of, in place of, for

$\ddot{\alpha} \xi_{105},-\alpha,-0 \nu$, fitting, worthy

$\varepsilon \dot{\pi} \pi \theta \cup \mu \varepsilon \hat{\varepsilon} \omega, I$ desire

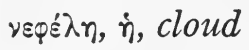

$\pi \dot{\alpha} \sigma \alpha \alpha$, $\sigma^{\prime}$ (indeclinable), the Passover

$\pi \dot{\alpha} \sigma \chi \omega, I$ suffer; second aorist हैं $\alpha \theta 0 \nu$

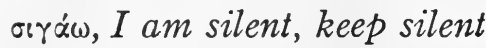

ouvero: $\omega, I$ eat with (someone)

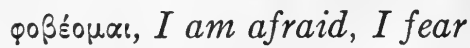

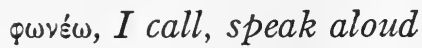

xpovith $\omega$, spend time, tarry

356. Learn the forms of the following irregular substantives of the third declension given in $\S \mathbf{I 2}$ :

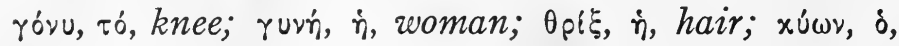

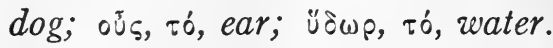

Note especially the forms not in parenthesesthe forms in parentheses do not occur in the New Testament.

357. The infinitive, as we have learned, has tense and voice; but it has no manner of affirmation and is not a mode.

There are a great many uses of the infinitive. Note carefully some of the uses of the infinitive in the following:

I. As an indeclinable verbal substantive (of 
neuter gender) the infinitive may be used in any case (not vocative) with or without the article. With the article it is indeclinable; but the neuter article with the infinitive is declined and shows the case of the infinitive.

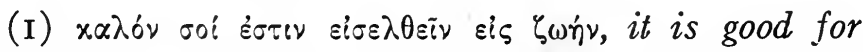

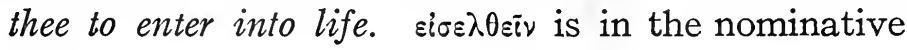
case.

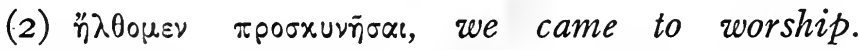
$\pi p 0 \sigma \times u \vee \bar{n} \sigma \alpha$ is in the dative case. In this construction the infinitive is common for the expression of purpose.

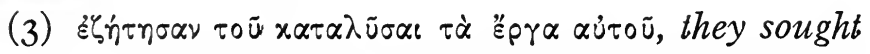

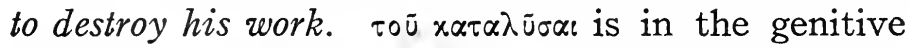
case. ro $\tilde{u}_{\mathrm{w}}$ and the infinitive is common in the New Testament to express purpose.

2. The infinitive with the article is used in most of the constructions in which any other substantive is used.

(I) The infinitive is used with such verbs

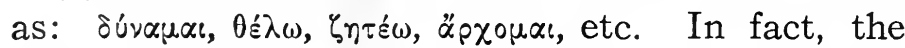
infinitive can be used with almost any verb that can be used with a substantive.

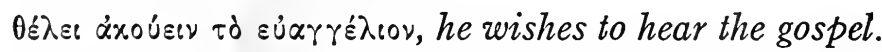

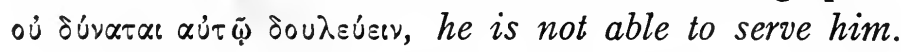

(2) The infinitive is used with substantives, most frequently with those expressing time, fitness, ability, need, etc.

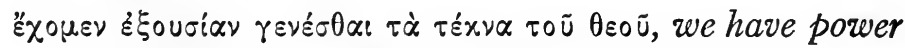
to become the children of God.

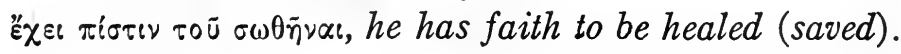


(3) The infinitive is used with adjectives, commonly with

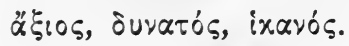

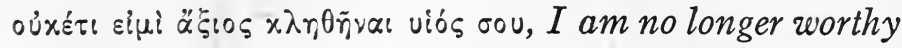
to be called a son of thine.

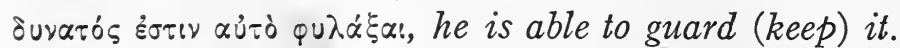

(4) The infinitive with the article is used with many prepositions.

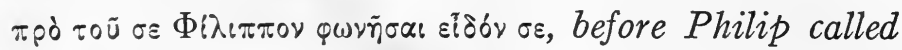
thee, I saw thee.

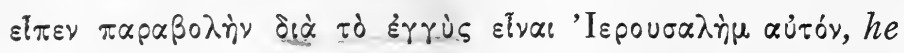

spoke a parable because he was near Jerusalem.

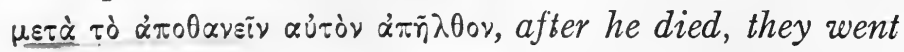
away.

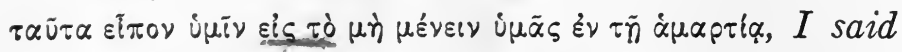
this to you/in order that you might not remain in sin.

Note: sis to and an infinitive is a common construction to express purpose.

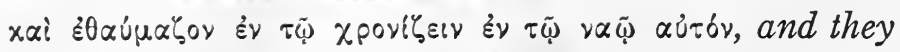
marvelled while he was tarrying in the temple.

$a$. Observe that the case of the infinitive has its proper meaning. The prepositions, as with cases of other substantives, help out the meaning of the cases. What the resultant meaning is depends on the meaning of the word, the case with the preposition, and the context. In the first example the resultant meaning of $\pi \rho \dot{\partial} \tau o \tilde{u} \varphi \omega v \tilde{n} \sigma \alpha$ (abl. case) is

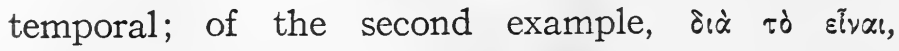
causal; of the third, $\mu \varepsilon \tau \dot{\alpha} \tau \dot{\delta} \alpha \pi 0 \theta \alpha v \varepsilon i \nu$, temporal; of

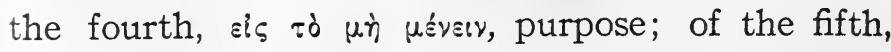




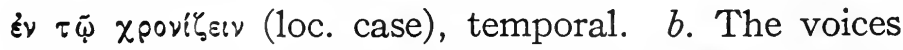
of the infinitive have the usual significance. $c$. The tenses have their force in the infinitive, as in the modes and participle. Tense in the infinitive has ro time except in indirect discourse. $d$. It is not necessary for the article to come next to the infinitive. Several words may intervene (see first example under (4)) and the clause may be one of considerable extent. $e$. The infinitive is not a mode and is not limited by personal endings; and, therefore, does not have a subject. See $327, a$.

358.

\section{EXERCISES}

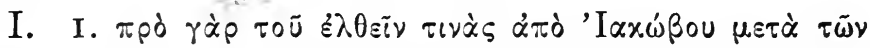

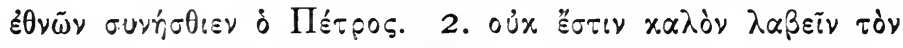

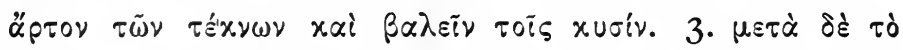

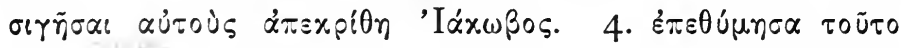

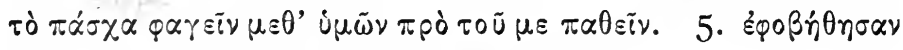

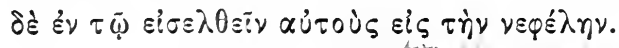

II. I. After he entered the house, the crowd went away. 2. While the woman was going away, he spoke to his disciples. 3. The son of man has power to save men. 4. He was worthy to receive the glory. 5. Before he saw you I called you.

\section{LESSON XLIII}

The Perfect and Pluperfect Indicative Active 359. VOCABULARY

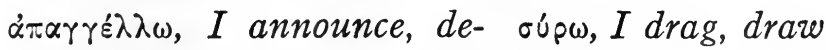
clare

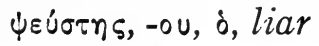


$\dddot{\varepsilon} \xi \omega$, adv., without; used also $\varphi \varepsilon u ́ \gamma \omega$, I flee, take flight;

with abl.

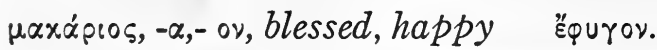

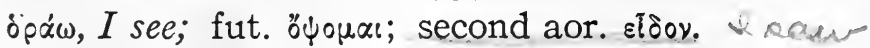

360. The perfect indicative active of $\lambda \dot{\omega} \omega$ is:

Singular

Plural

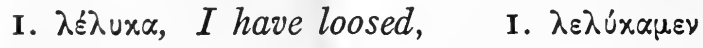

2. $\lambda \varepsilon^{\prime} \lambda \cup \times \alpha \varsigma$ etc.

2. $\lambda \varepsilon \lambda \dot{u} x \alpha \tau \varepsilon$

3. $\lambda \varepsilon \dot{\lambda} \cup x \varepsilon$

3. $\lambda \varepsilon \lambda \dot{u} x \alpha \sigma \iota$ or $\lambda \varepsilon \dot{\varepsilon} \lambda u x \alpha \nu$

Perfect active infinitive, $\lambda \varepsilon \lambda u x \varepsilon \dot{\varepsilon}$ a

$a$. In the second pers. sing. a form like $\lambda \varepsilon \hat{\varepsilon} u x \varepsilon s$ occurs a few times. $b$. In the third pers. plur. a form like $\lambda \varepsilon^{\prime} u_{u x \alpha \nu}$ occurs ten times in the New Testament.

361. Observe: I. To the verb-stem ( $\lambda u-)$ is prefixed its initial consonant $(\lambda)$ with $\varepsilon$. This is called reduplication. 2. The suffix $-x \alpha$ is added to the reduplicated verb-stem: thus is formed, in the indicative, the first (or $x$ ) perfect stem (active). 3. The personal endings seem to be the secondary personal endings, except $-\alpha \sigma$ in the third plur.

362. The first (or $x$ ) perfect (active) is generally formed from verb-stems ending in a vowel, a liquid $(\lambda, \rho)$, or a lingual (dental) mute $(\tau, \delta, \theta)$.

I. Vowel stems. If the final vowel of the stem is long (or a diphthong) $-x \alpha$ is added to the reduplicated verb-stem without change, as $\pi \varepsilon \pi i \sigma \tau \varepsilon u \times \alpha$.

If the final vowel of the stem is short, it is lengthened before $-x \alpha$ as $v i x \dot{\alpha} \omega, I$ conquer, $\nu \varepsilon v i x \eta x \alpha$; $\pi 0$ of $\omega$, $I d o, \pi \varepsilon \pi 0 i \eta x \alpha ; \pi \lambda \eta p b \omega, \pi \varepsilon \pi \lambda \dot{\eta} \rho \omega x \alpha$.

Here $\alpha, \varepsilon$, and o follow the same principle of length- 
ening as in the future and first aorist active and passive.

2. Liquid stems. $-x \alpha$ is added to the reduplicated verb-stem, as $\sigma \tau \hat{\varepsilon} \lambda \lambda \omega$ ( $\sigma \tau \alpha \lambda-$ ), है $\sigma \tau \alpha \lambda x \alpha \alpha$ (for reduplication see below).

3. Lingual mute stems. $\tau, \delta$, or $\theta$ is dropped before $-x \alpha$, as $\sigma \dot{\prime} \zeta \omega(\sigma \omega \delta)$, $\sigma \varepsilon \dot{\delta} \sigma x \alpha$.

363. Verbs beginning with a vowel, two consonants (except a mute and liquid), a double consonant

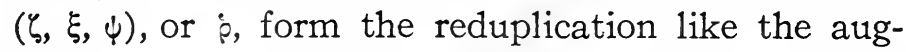
ment. $\rho$ is generally doubled. Thus: $\dot{\alpha} \gamma \alpha \pi \dot{\alpha} \omega$,

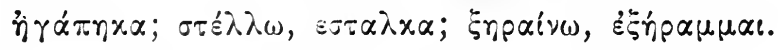

364. Verbs beginning with a rough mute $(\varphi, \chi, \theta)$ have the corresponding smooth mute $(\pi, x, \tau)$, in re-

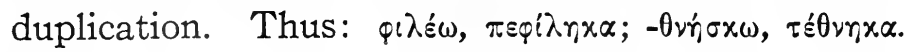
365. A few verbs have a seemingly irregular perfect, as

$\gamma(\nu \omega ́ \sigma x(\omega)$, perf. act. "̋ $\gamma \nu \omega x \alpha$.

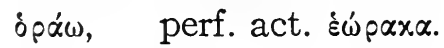

366. In formation of the perfect active a few verbs add $-\alpha$ and not $-x \alpha$ to the reduplicated verb-stem. These are called second or strong perfects, and are older than the $x$-perfects. Conj. like $x$ - perf.

\begin{tabular}{|c|c|}
\hline $\begin{array}{l}\text { Present } \\
\dot{\alpha} \times 0 \text { ú }\end{array}$ & 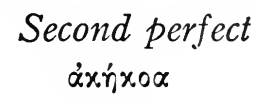 \\
\hline$\gamma^{\prime}(v \circ \mu \alpha \ell$ & 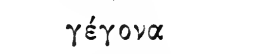 \\
\hline 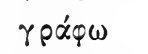 & $\gamma^{\prime} \gamma \rho \alpha \varphi \alpha$ \\
\hline 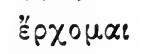 & $\varepsilon \lambda \dot{\eta}^{\prime} \lambda \cup \theta \alpha$ \\
\hline$\pi \dot{\alpha} \sigma \chi \omega$ & $\pi \varepsilon ́ \pi \circ \nu \theta \alpha$ \\
\hline$\pi \varepsilon i \theta \omega$ & $\pi \varepsilon ́ \pi \circ: \theta \alpha$ \\
\hline$\varphi \varepsilon u ́ \gamma \omega$ & $\pi \dot{\varepsilon} \emptyset \varepsilon \cup \gamma \alpha$. \\
\hline
\end{tabular}


367. The tense in Greek called perfect is really a present perfect. The perfect presents the action of the verb in a completed state or condition. When the action was completed the perfect tense does not tell. It is still complete at the time of the use of the tense by the speaker or writer. The perfect expresses the continuance of completed action. It is then a combination of punctiliar action and durative action. This kind of action expressed by the perfect tense is sometimes called perfective action.

368. The perfect tense as tense is timeless. But in the indicative the time element is present. The perfect indicative generally expresses the present result of a past action. It then has to do with the past and the present. The English perfect is not an equivalent to the Greek perfect. The translations given in the paradigms are not at all to be taken as equivalent to the Greek perfect, but as a means of associating the verb meaning with the verb. Aktionsart of the verb applies to the perfect. E.g., $\gamma^{\prime} \gamma p \alpha \rho, \alpha, I$ wrote and the statement is still on record. $\varepsilon \lambda \hat{\eta} \lambda \cup \theta \alpha$, I came (punctiliar) and am still here (durative).

369. The pluperfect (past perfect) indicative active of $\lambda \dot{v} \omega$ is

Singular Plural

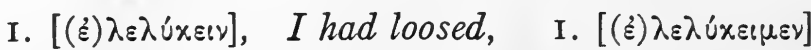

2. $[(\varepsilon) \lambda \varepsilon \lambda \cup$ us: $]$ etc.

2. (๕) $\lambda \varepsilon \lambda u ́ x \varepsilon i \tau \varepsilon$

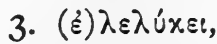

3. (๕) $\lambda \varepsilon \lambda u ́ x \varepsilon \varepsilon \sigma \alpha \nu$

$a$. The forms in brackets do not occur in the New Testament. 


\section{PLUPERFECT INDICATIVE}

Observe: I. The pluperf. is made upon the perfect stem. 2. The thematic vowel is $\varepsilon$. 3. The personal endings are the secondary personal endings. 4. There is an augment before the reduplication. The augment is usually dropped in the pluperf. in the New Testament.

370. If a second perfect has a pluperfect, the pluperf. is made on the second perfect stem and is called the second pluperfect. Thus:

Second pluperf.

\begin{tabular}{|c|c|c|}
\hline & Second perf & (third pers. si \\
\hline & rérova & $\Rightarrow$ \\
\hline
\end{tabular}

The pluperfect expresses continuance of the completed state in past time up to a prescribed limit in the past.

371.

EXERCISES

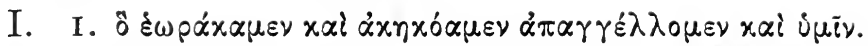

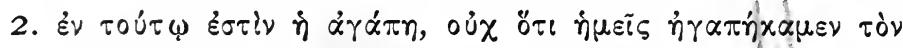

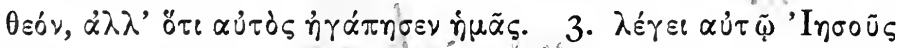

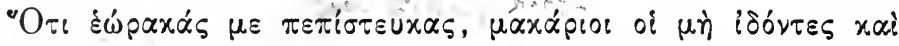

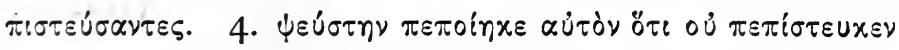

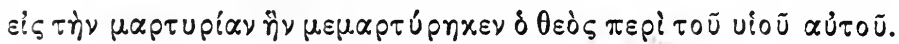

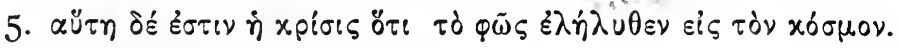

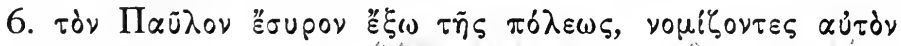
$\tau \varepsilon \theta \nu \eta x \varepsilon ́ v \alpha$. to

II. I. We have made him King. 2. He said "What I have written, I have written." 3. We have kept the faith. 4. I have come to do the will of him who sent me. 


\section{LESSON XLIV}

\section{The Perfect Tense (Continued)}

372.

E̊prí, $\dot{\eta}$, feast

$\ddot{\eta} \lambda$ เos, $\delta$, sun

$\theta \varepsilon p \alpha \pi \varepsilon \dot{v} \omega$, I heal

$\varepsilon \mu o ́ s,-\dot{\eta},-o ́ v$, poss. pron.

of first pers. $m y$, mine
VOCABULARY

obs,- $\dot{\eta},-\delta \nu$, poss. pron. of the second pers., thy, thine jijérepos, $-\alpha,-0 \nu$, poss. pron. of the first pers., our

ưsérpos, $-\alpha,-\circ \nu$, poss. pron. of the second pers., your

373. The declension of the perfect active participle of $\lambda u \omega$ is

\section{Singular}

Masc.

Nom. $\lambda \varepsilon \lambda u x \omega ́ s$

Gen. $\} \quad \lambda \varepsilon \lambda$ uxóros

Abl.

Loc.

Ins.

Dat.

Acc.

$\lambda \varepsilon \lambda \cup \times \sigma^{2} \tau t$

$\lambda \varepsilon \lambda \cup \times \sigma_{0}=\alpha$

$\lambda \varepsilon \lambda u \times o ́ \tau \varepsilon \varsigma$

Nom

Gen.

Abl.

$\lambda \varepsilon \lambda u \times o ́ \tau \omega \nu$

Loc.

Ins.

Dat.

Acc.

$$
\text { Fem. }
$$

$\lambda \varepsilon \lambda \cup x \cup \operatorname{Li\alpha }$

( $\lambda \varepsilon^{\prime} \lambda_{u} \times u^{\prime} \alpha \varsigma$

$\lambda \varepsilon \lambda \cup \times$ víq

$\lambda \varepsilon \lambda \cup x \cup \bar{\alpha} \alpha$

Plural

\begin{tabular}{|c|c|}
\hline$\lambda \varepsilon \lambda \cup x \cup \bar{\alpha} \alpha !$ & $\lambda \varepsilon \lambda \cup \times o ́ \tau \sigma \alpha$ \\
\hline$\lambda \varepsilon \lambda \cup x \cup ! \tilde{\omega} \nu$ & $\lambda \varepsilon \lambda u \times o ́ \tau \omega \nu$ \\
\hline$\lambda \varepsilon \lambda \cup x u i \alpha i s$ & $\lambda \varepsilon \lambda \cup \times \dot{\sigma} \sigma$ \\
\hline$\lambda \varepsilon \lambda \cup x u i \alpha \varsigma$ & $\lambda \varepsilon \lambda \cup \times o ́ \tau \alpha$ \\
\hline
\end{tabular}

Neut.

$\lambda \varepsilon \lambda \cup \times 6 \varsigma$

$\lambda \varepsilon \lambda \cup \times o ́ \tau 0 \varsigma$

$\lambda \varepsilon \lambda u$ xó $\tau$

$\lambda \varepsilon \lambda \cup \times o ́ s$ 
Observe: I. The perfect act. part. is made on the perfect act. stem. 2. In the masc. and neut. it is declined according to the third declension, and in the fem. according to the first declension. 3. The accent seemingly is irregular.

374. The second perfect act. part. is made on the second perfect act. stem, and is commonly declined like $\lambda \varepsilon \lambda u x \omega ́ s,-x \cup i \alpha$, -xós. Thus,

Present Second perf. Second perf. part.

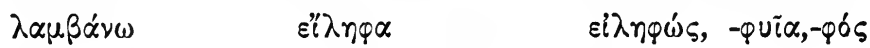

375. The perfect indicative middle and passive of $\lambda \dot{u} \omega$ is:
Singular
Plural
I. $\lambda \varepsilon ́ \lambda \cup \mu \alpha \ell$
I. $\lambda \varepsilon \lambda \cup u \varepsilon \varepsilon \theta \alpha$

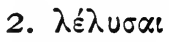
2. $\lambda \varepsilon \dot{\varepsilon} \lambda \cup \sigma \varepsilon$

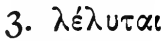

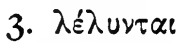

Perf. midd. and pass. infinitive, $\lambda \varepsilon \lambda \cup \operatorname{\sigma } \theta \alpha \iota$

Observe: I. The stem of the perf. middle and pass. is the reduplicated verb-stem. 2. The primary middle personal endings are attached directly to the reduplicated verb-stem. 3. There is no thematic vowel.

a. The translations I have loosed (for) myself (midd.) and I have been loosed (pass.) do not give accurately the meaning of the perfect tense in Greek.

376. Verbs with stems in a short vowel $(\alpha, \varepsilon, 0)$ lengthen the vowel (as in the fut., aor., etc.) before the personal endings, as,

$\pi \lambda \eta p o ́ \omega, \pi \varepsilon \pi \lambda \hat{\eta} \rho \omega \mu \alpha \iota ; \theta \varepsilon \dot{\alpha} \sigma \mu \alpha \ell, \tau \varepsilon \theta \varepsilon \dot{\varepsilon} \alpha \alpha \iota$. 
377. Of verbs with stems in a labial mute $(\pi, \beta, \varphi)$, a palatal mute $(x, \gamma, \chi)$, a liquid $(\lambda, \rho)$, or a nasal $(\mu, \nu)$, the third pers. sing. only of the perf. middle and passive occurs in the New Testament. Of verbs with stems in a lingual mute $(\tau, \delta, \theta)$ the first and third pers. sing. and the first pers. plur. occur in the New Testament. See p. 240, §3I.

378. The pluperfect indicative middle (and passive) is formed by adding the secondary middle endings to the perfect middle stem. Sometimes there is also an augment. The pluperf. indic. middle third pers. plur. of $\lambda \dot{u} \omega$ is (z) $\lambda \varepsilon \dot{\varepsilon} \lambda u \nu \tau o$. The pluperfect indic. middle (and passive) of verbs with stems in a consonant is so rare that the forms are not given here.

379. The perfect middle (and passive) participle is formed by adding the middle participle endings,

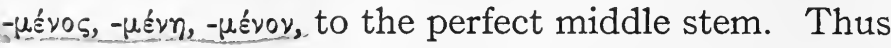
the perfect middle (and passive) participle of $\lambda \dot{v} \omega$ is:

$$
\begin{aligned}
& \text { Masc. Fem. Neut. }
\end{aligned}
$$

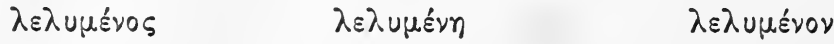

With the exception of the accent, it is declined like other participles in $\mu \varepsilon v \circ \varsigma,-\eta,-o \nu$.

380. It needs to be remembered that perfective action runs through the whole perfect tense, and that Aktionsart of the verb-stem modifies the tense action.

The perf. participle, then, may represent a state or a completed action. The time of the perf. part. is relative to the time of the principal verb. The action of the participle may be: 
I. Coincident with that of the principal verb; e.g.,

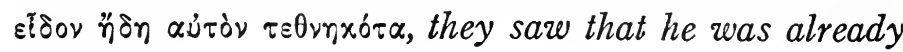
dead.

2. Antecedent to that of the principal verb; e.g.,

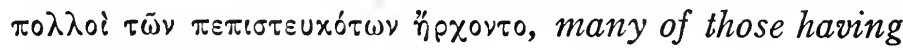
believed were coming.

The perf. part. may be used in any of the constructions in which other participles are used.

381. The second class conditional sentence. It is the condition determined as unfulfilled. The condition is assumed to be contrary to fact. The thing in itself may be true, but it is treated as untrue. The condition has only to do with the statement, not with the actual fact. The condition has $\varepsilon i+a$ past tense of the indicative; the conclusion has a past tense of the indicative usually with $\ddot{\alpha}$.

$\ddot{\nu}$ cannot be really translated into English. It seems to have a definite sense, in that case, and an indefinite sense. It is a particle used to give more color to the mode with which it occurs.

I. The imperf. ind. is used in unfulfilled conditions about present time; e.g.,

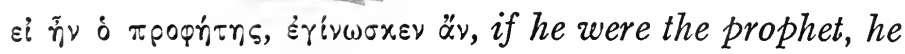
would know.

2. The aorist ind. or pluperf. ind. is used in unfulfilled conditions about past time; e.g.,

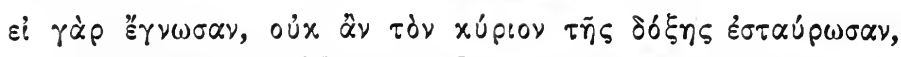
for if they had known, they would not have crucified the Lord of glory.

a. Sometimes one tense occurs in one clause, 
anciner tense in the other clause. $b$. Each tense has its proper kind of action. $c$. The negative with the protasis (if-clause) is $\mu \dot{n}$, with the apodosis ou.

382.

\section{EXERCISES}

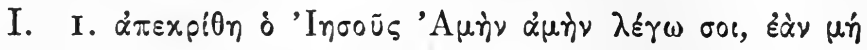

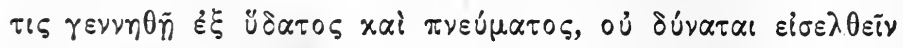

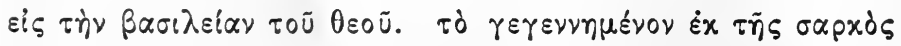

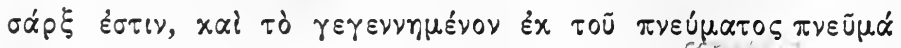

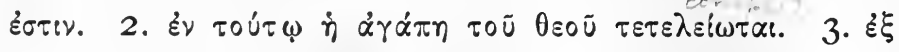

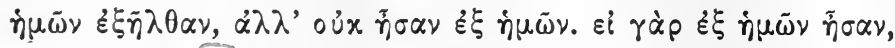

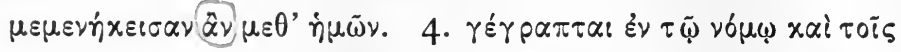

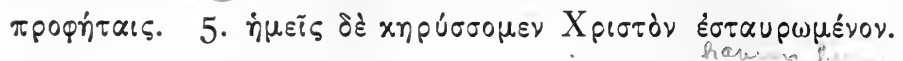

II. I. If we had seen him, we would have loved him. 2. The days have been fulfilled. 3. We receive those who have believed on the Lord.

\section{LESSON XLV}

\section{Reflexive Pronoun. Reciprocal Pronoun.}

\section{Indefinite Relative Pronoun}

383.

VOCABULARY

$\varepsilon \lambda \pi i \zeta \omega$, I hope

ò $\pi \lambda$ noiov, neighbor

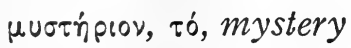

$\pi \lambda$ noiov, adv., near

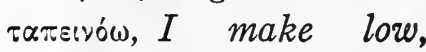
humble

384. The reflexive pronouns are:

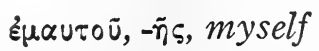

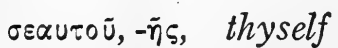

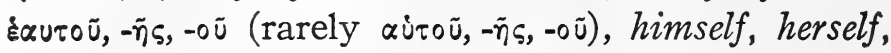
itself 
They are declined as follows:

I. First person:

\begin{tabular}{|c|c|c|c|c|}
\hline & \multicolumn{2}{|c|}{ Singular } & \multicolumn{2}{|c|}{ Plural } \\
\hline $\begin{array}{l}\text { Gen. } \\
\text { Abl. }\end{array}$ & $\begin{array}{l}\text { Masc. } \\
\varepsilon \dot{\mu} \alpha \cup \tau o \tilde{u}\end{array}$ & $\begin{array}{l}\text { Fem. } \\
\varepsilon \varepsilon \mu \alpha \cup \tau \tilde{\eta} s\end{array}$ & 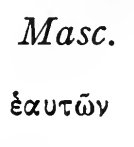 & $\begin{array}{l}\text { Fem. } \\
\dot{\varepsilon} \alpha \cup \tau \tilde{\omega} \nu\end{array}$ \\
\hline $\begin{array}{l}\text { Loc. } \\
\text { Ins. } \\
\text { Dat. }\end{array}$ & $\varepsilon \dot{\mu} \alpha \cup \tau \bar{\varphi}$ & $\dot{\varepsilon} \mu \alpha \cup \tau \tilde{n}$ & $\dot{\varepsilon} \alpha \cup \tau 0 i s$ & 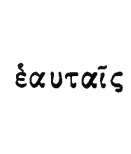 \\
\hline Acc. & 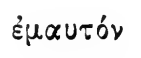 & $\varepsilon \mu \alpha u \tau \eta \dot{\eta} \nu$ & 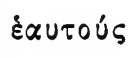 & $\dot{\varepsilon} \alpha \cup \tau \alpha \dot{s} \varsigma$ \\
\hline
\end{tabular}

2. Second person:

\section{Singular}

\section{Plural}

\begin{tabular}{|c|c|c|c|c|}
\hline & Masc. & Fem. & Masc. & Fem. \\
\hline $\begin{array}{l}\text { Gen. } \\
\text { Abl. }\end{array}$ & \}$\sigma \varepsilon \alpha \cup \tau 0 \tilde{U}$ & $\sigma \varepsilon \alpha \cup \tau \bar{\eta} \varsigma$ & $\grave{\varepsilon} \alpha u \tau \tilde{\omega} \nu$ & $\dot{\varepsilon} \alpha u \tau \bar{\omega} \nu$ \\
\hline $\begin{array}{l}\text { Loc. } \\
\text { Ins. } \\
\text { Dat. }\end{array}$ & $\sigma \varepsilon \varepsilon \cup \cup \bar{\varphi}$ & $\sigma \varepsilon \alpha \cup \tau \tilde{n}$ & $\varepsilon \alpha \cup \tau 0 i \varsigma$ & $\varepsilon \alpha \cup \tau \alpha i \varsigma$ \\
\hline Acc. & 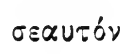 & $\sigma \varepsilon \alpha u \tau \dot{\eta} \nu$ & 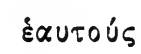 & $\dot{\varepsilon} \alpha u \tau \alpha^{\prime} \varsigma$ \\
\hline
\end{tabular}

3. Third person:

\begin{tabular}{|c|c|c|c|}
\hline & & Singul & \\
\hline & Masc. & Fem. & Neut. \\
\hline $\begin{array}{l}\text { Gen. } \\
\text { Abl. }\end{array}$ & \}$\dot{\varepsilon} \alpha u \tau \sigma u \bar{~}$ & $\varepsilon \alpha u \tau \tilde{\eta} \varsigma$ & $\dot{\varepsilon} \alpha \cup \tau \circ \tilde{U}$ \\
\hline $\begin{array}{l}\text { Loc. } \\
\text { Ins. } \\
\text { Dat. }\end{array}$ & $\xi \varepsilon \cup \cup \tau \bar{\varphi}$ & $\varepsilon \alpha \cup \tau \tilde{n}$ & $\dot{\varepsilon} \alpha \cup \tau \tilde{\varphi}$ \\
\hline Acc. & $\varepsilon \alpha u \tau \delta \nu$ & $\dot{\varepsilon} \alpha \cup \tau \dot{\eta} \nu$ & $\dot{\varepsilon} \alpha \cup \tau \delta$ \\
\hline
\end{tabular}




\section{Plural}

\begin{tabular}{|c|c|c|c|}
\hline & Masc. & Fem. & Neut. \\
\hline $\begin{array}{l}\text { Gen. } \\
\text { Abl. }\end{array}$ & $\dot{\varepsilon} \alpha u \tau \bar{\omega} \nu$ & $\dot{\varepsilon} \alpha \cup \tau \bar{\omega} \nu$ & $\dot{\varepsilon} \alpha \cup \tau \tilde{\omega} \nu$ \\
\hline $\begin{array}{l}\text { Loc. } \\
\text { Ins. } \\
\text { Dat. }\end{array}$ & 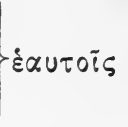 & $\dot{\varepsilon} \alpha u \tau \alpha i \bar{\varphi}$ & $\varepsilon \alpha \cup \tau 0 i \varsigma$ \\
\hline Acc. & 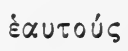 & $\dot{\varepsilon} \alpha u \tau \alpha \dot{\alpha}$ & $\dot{\varepsilon} \alpha u=\dot{\alpha}$ \\
\hline
\end{tabular}

Observe: I. These pronouns occur in the oblique cases only. 2. They are formed from the personal pronouns plus aisós. 3. There is no neuter gender in the reflexive pronouns of the first and second persons. 4. The plural form is the same for all three persons.

385. Examine carefully the following examples of the use of the reflexive pronouns.

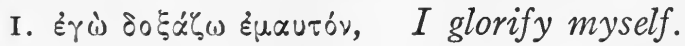

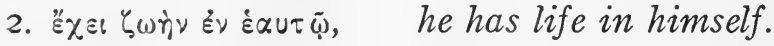

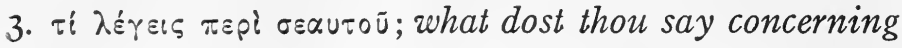
thyself?

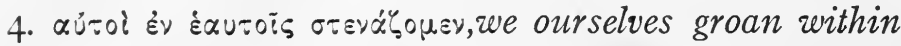
ourselves.

In these examples it is seen that the pronoun refers back to the subject of the clause, hence the name reflexive.

386. The reciprocal pronoun is $\alpha \lambda \lambda \dot{\eta} \lambda \omega \nu$, of one another. It occurs in the New Testament only in the masculine forms $\dot{\alpha} \lambda \lambda \dot{\eta} \lambda \omega \nu, \dot{\alpha} \lambda \lambda \dot{\eta} \lambda$ oเ,$\dot{\alpha} \lambda \lambda \dot{\eta} \lambda$ ous. No fem. and neut. forms occur.

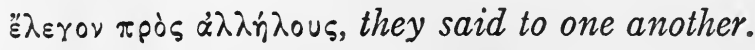


387. oij $\alpha, I$ know, an old perfect with a present (durative) meaning is conjugated in the indicative active as follows:

Singular

Plural
I. $0 i \delta \alpha$,
I know,

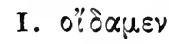
2. $0 . \delta \alpha \varsigma$ etc.
2. o"ठ $\alpha \tau \varepsilon$
3. oĩ $\varepsilon$
3. $0 \% \delta \alpha \sigma t$

Infinitive, sióśva

388. The forms of the (indefinite) relative pronoun

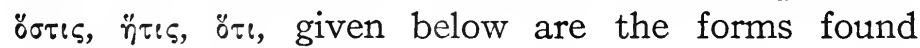
in the New Testament.

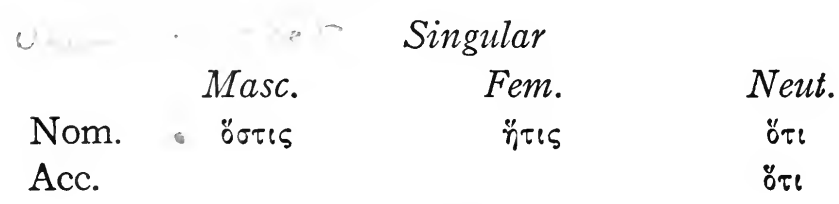

\section{Plural}

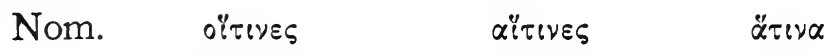

$a$. An old form örou (gen.) is found in certain set phrases.

This pronoun is made from the relative os and the indefinite $\tau$ is. It is used with the meanings "anyone" (indefinite) and "somebody" in particular (definite), i.e., "whoever" (indef.) and "who" (def.). 389.

EXERCISES

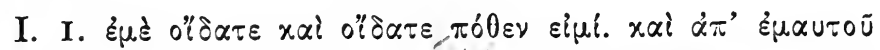

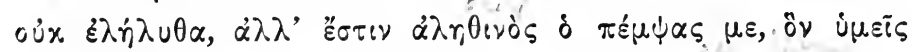

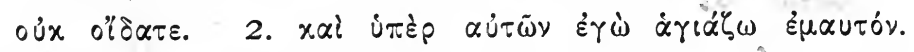

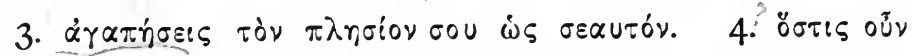

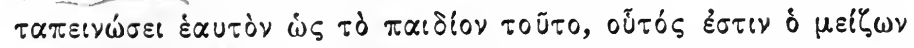

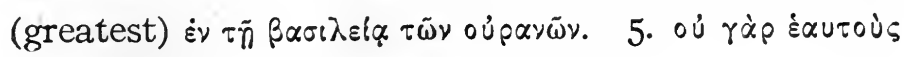




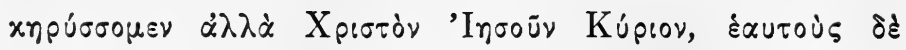

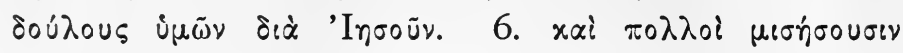
$\dot{\alpha} \lambda \lambda \dot{n} \lambda$ ous.

II. I. You shall love one another. 2. I have not spoken concerning myself. 3. We preached not ourselves, but Christ. 4. They said that he made himself the son of God.

\section{LESSON XIVI}

Future and Aorist Active and Middle of Liquid Verbs 390. VOCABULARY

$\dot{\alpha} \pi 0 x \tau$ siv(s), I kill, slay

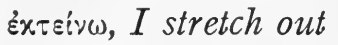

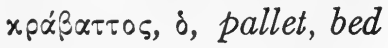

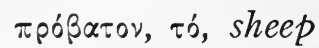

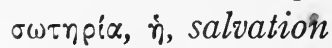

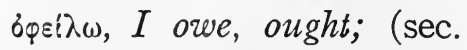
aorist without augment. "øष

391. Verbs with stems in a liquid $(\lambda, \nu, \rho)$ form the futures by adding $-\varepsilon \circ / \varepsilon^{1}$ to the stem. The $\varepsilon$ of the suffix contracts with the thematic vowel o/ . (Remember that the present stam is not always the same as the verb-stem.)

392. The conjugation of xpiva in the future indicative is:

\section{Active}

Singular

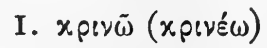

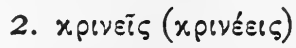

3. хрเขยі (
Plural

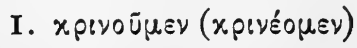

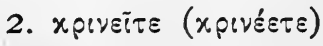

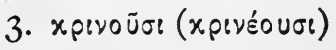

1 Originally $\varepsilon \sigma \% / \varepsilon$. $\sigma$ was expelled. 


\section{MidDle}

\section{Singular}

\section{Plural}

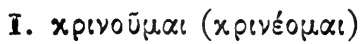
I. $x \rho\llcorner v o u ́ u \varepsilon \theta \alpha \quad(x \rho\llcorner v \varepsilon b \mu \varepsilon \theta \alpha)$
2. $x$ p เขñ (xpเvén)

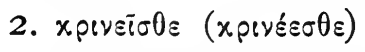

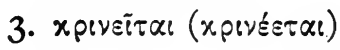

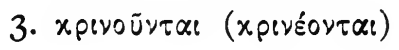

Observe that when one of the uncontracted syllables has an accent the contracted form has an accent (the circumflex, if possible).

$a$. The uncontracted forms do not occur. They are given to exhibit the vowels that are contracted.

b. Table of the vowel contractions.

$$
\begin{array}{ll}
\varepsilon+\omega=\omega & \varepsilon+0=0 u \\
\varepsilon+\varepsilon l=\varepsilon ! & \varepsilon+0 u=0 u \\
\varepsilon+\varepsilon+\varepsilon \iota &
\end{array}
$$

393. Verbs with stems in a liquid form the aorist active and middle by lengthening the stem vowel and adding ${ }_{-} \alpha^{1} \quad \alpha$ of the stem is lengthened to $\eta$ (but $\bar{\alpha}$ before $\rho$ ), $\varepsilon$ to $\varepsilon \iota, \check{\imath}$ to $\iota$ and $\check{u}$ to $\bar{u}$. The conjugation through all the modes, infinitives, and participles is like the regular first aorist; thus the aor. ind. act. of $\mu \varepsilon \dot{\varepsilon} \omega \omega$ is "s $\mu \varepsilon \imath \nu \alpha$, etc.; the subj.,

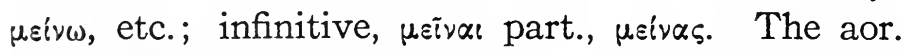

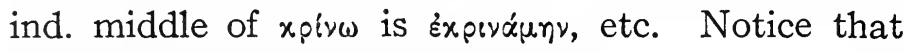
these verbs have no $\sigma$ in the aorist.

394. Liquid verbs may have a second aorist, as

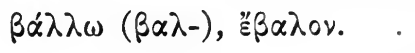

1 Originally $\sigma$ was expelled after a liquid. 
395. Learn the stem, the future, and aorist of the following verbs:

\begin{tabular}{|c|c|c|c|}
\hline Present & Stem & Future & Aorist act. \\
\hline$\dot{\alpha} \gamma \gamma \hat{\varepsilon} \lambda \lambda \omega \omega$ & $\dot{\alpha} \gamma \gamma \varepsilon \lambda-$ & $\dot{\alpha} \gamma \gamma \varepsilon \lambda \bar{\omega}$ & $\ddot{\eta} \gamma \gamma \xi \varepsilon i \lambda \alpha$ \\
\hline$\alpha l p \omega$ & $\dot{\alpha p-}$ & $\dot{\alpha} \rho \tilde{\omega}$ & $\dot{n} \rho \alpha$ \\
\hline$\dot{\alpha} \pi 0 x \tau \operatorname{sin\omega }$ & $(\dot{\alpha} \pi 0) x \tau \varepsilon \nu-$ & $\dot{\alpha} \pi 0 x \tau \varepsilon \nu \tilde{\omega}$ & $\dot{\alpha} \pi \varepsilon ́ x \tau \varepsilon เ \nu \alpha$ \\
\hline$\dot{\alpha} \pi 0 \sigma \tau \varepsilon \hat{\varepsilon} \lambda \lambda \omega$ & $(\dot{\alpha} \pi 0) \sigma \tau \varepsilon \lambda-$ & $\dot{\alpha} \pi 0 \sigma \tau \varepsilon \lambda \tilde{\omega}$ & $\alpha \dot{\alpha} \pi \hat{\sigma} \sigma \tau \varepsilon ! \lambda \alpha$ \\
\hline$\beta \alpha^{\prime} \lambda \lambda \omega$ & $\beta \alpha \lambda-$ & $\beta \alpha \lambda \tilde{\omega}$ & 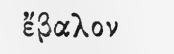 \\
\hline$\varepsilon \dot{\gamma} \xi i \rho \omega$ & $\varepsilon \gamma \gamma \varepsilon \rho-$ & $\varepsilon \dot{\xi} \varepsilon \rho \tilde{\omega}$ & $\ddot{\eta} \gamma \varepsilon ı \rho \alpha$ \\
\hline
\end{tabular}

Of course the.compound forms of these verbs form their tenses in the same way as the uncompounded forms, as

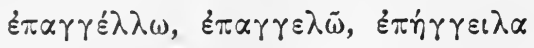
$\dot{\alpha} \pi \circ x \tau \varepsilon i v \omega$ and $\dot{\alpha} \pi \circ \sigma \tau \dot{\varepsilon} \lambda \lambda(\omega)$ are compound verbs.

396. The tenses of the verb in Greek are divided into nine tense-systems. As we have seen each tense-system has a distinct stem, called tense-stem.

Systems

Tenses

I. Present, including present and imperfect in all voices.

2. Future,

“ future active and middle.

3. First aorist,

“ first aorist act. and middle (and liquid aorists also).

4. Second aorist, “ second aorist act. and middle.

5. First perfect, “ first perfect and pluperf. active.

6. Second perfect, “ second perfect and pluperf. active. 
7. Perfect middle, including perfect and pluperf. middle and passive (and future perfect).

8. First passive, " first aorist and future passive.

9. Second passive, “ second aorist and future passive.

a. Most verbs have only six of these nine systems, since very few verbs have both the first and second forms of the same tense; many verbs have less than six. No verb occurring in the New Testament is used in all nine systems.

397. The principal parts of a Greek verb are the first person singular indicative of every system used in it; e.g.,

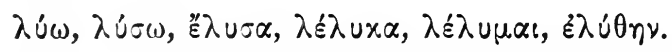

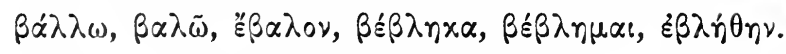

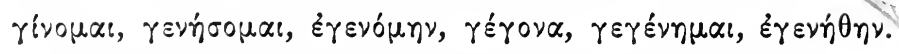

To know a verb one must know its principal parts.

398.

EXERCISES

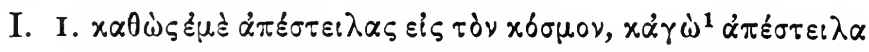

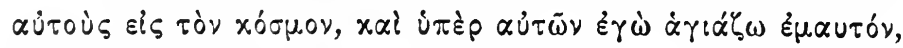

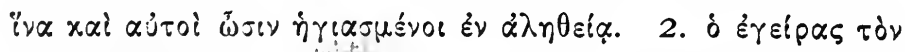
'I

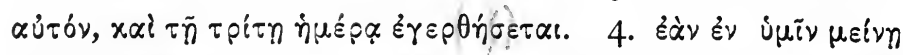

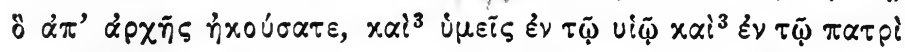

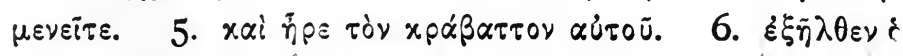

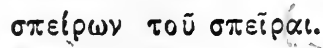

$\left.{ }^{1} x \dot{\alpha} \gamma \omega\right)=x \alpha \ell$ है $\gamma \dot{\omega}$, see $\S 4$.

${ }^{2}$ Associative-ins. case. ${ }^{3}$ See $\gamma \alpha$ ? . . x $\alpha$ l in 415. 
II. I. They sought to kill him. 2. The disciples sowed the word. 3. I will send unto them prophets and apostles. 4. If we remain in the truth, the truth will remain in us.

\section{LESSON XLVII}

\section{The Imperative Mode}

399.

VOCABULARY

äpхoual, I begin

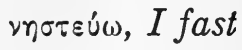

vaós, ó, temple

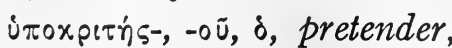

hypocrite

400. The imperative is comparatively a late development in Greek. More of its forms came from an old injunctive mode than from any other source.

401. The personal endings of the imperative are:

Active

Singular

2. $-,-\theta_{l},-\varsigma$

3. $-\tau \omega$.

Middle (And Passive)

2. $-\sigma 0$

3. $-\sigma \theta \omega$
Plural

$-\tau \varepsilon$

$-\tau \omega \sigma \alpha \nu$.

$-\sigma \theta \varepsilon$

$-\sigma \theta \omega \sigma \alpha \nu$.

a. $-\theta_{l}$ (probably an old adverb) is found in a few old verbs, and, except in the aor. pass., is not used in the active forms of the imperative of most verbs. The ending $-\varsigma$ is found only in a few old verbs. $b$. $-\tau \omega$ is probably an old ablative form of a demonstrative pronoun. 
402. The present imperative active, middle, and passive is made on the present tense-stem. See 396.

403. The present active imperative of $\lambda \dot{u} \omega$ is:

\section{Singular}

2. $\lambda \tilde{\mathrm{u}}, \quad$ loose (thou)

3. $\lambda \cup \varepsilon \tau \omega$, let him loose

a. $\lambda \tilde{u} \varepsilon$ is the verb-stem with the thematic vowel $\varepsilon$. Note the thematic vowel $\varepsilon$ in all persons.

404. The present middle imperative of $\lambda \dot{\omega} \omega$ is:

Singular

Plural

2. úou, loose (for) thyself

2. $\lambda \cup ́ \varepsilon \sigma \theta \varepsilon$, loose (for) yourself

3. $\lambda \cup \varepsilon ์ \sigma \theta \omega$, let him loose

(for) himself

2. $\lambda u ́ \varepsilon \tau \varepsilon, \quad l o o s e(y e)$

3. $\lambda \cup \varepsilon ́ \tau \omega \sigma \alpha \nu$, let them loose

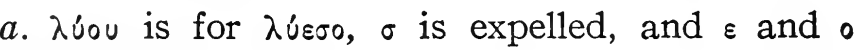
contract to ou.

405. The present passive imperative of $\lambda \dot{v} \omega$ is:

Singular

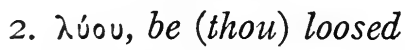

3. $\lambda$ uś $\theta \omega$, let him be loosed
Plural

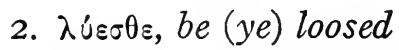

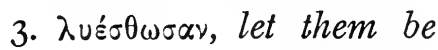
loosed

Observe that the pres. passive imperative is like the pres. middle in form.

406. The second aorist active imperative of $\beta \alpha^{\prime} \lambda \lambda \omega$ is:

Singular

2. $\beta \alpha \dot{\lambda} \lambda \varepsilon$

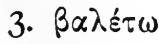

Plural

2. $\beta \alpha \alpha^{\prime} \lambda \varepsilon \tau \varepsilon$

3. $\beta \alpha \lambda \lambda^{\prime} \tau \omega \sigma \alpha \nu$ 
a. The second pers. sing. of some second aorists

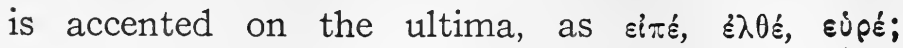
accent of imps. recessive. $b$. No augment.

407. The second aorist middle imperative of $\beta \dot{\alpha} \lambda \lambda \omega$ is

Singular

2. $\beta \alpha \lambda \circ \tilde{U}$

3. $\beta \alpha \lambda \varepsilon \dot{\varepsilon} \sigma \theta \omega$

\section{Plural}

2. $\beta \alpha^{\prime} \lambda \varepsilon \sigma \theta \varepsilon$

3. $\beta \alpha \lambda \varepsilon \varepsilon \theta \omega \sigma \alpha \nu$

a. Note the accent of $\beta \alpha \lambda_{0}$ u.

Observe that in the endings the imperative of the second aor. act. and middle is like that of the present act. and middle. The difference between them is the difference in tense-stem: the present tensestem is $\beta \alpha \lambda \lambda$-, the second aorist tense-stem is $\beta \alpha \lambda$ -

408. The imperative, like the subjunctive, is always future in time, though it may apply to the immediate future.

The difference in meaning between the present imperative and the aorist imperative is in the kind of action,-durative action in the present, and punctiliar - action in the aorist. The pres. imperative, then, has to do with action in progress. The aorist imperative has to do with the simple act without regard to progress. E.g.,

$\beta \dot{\alpha} \lambda \lambda \varepsilon \lambda_{i} \theta_{0}$ s, keep on (or go on) throwing stones. $\mu \dot{\eta} \beta \alpha \lambda \lambda \varepsilon$, stop (or quit) throwing stones.

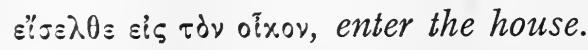

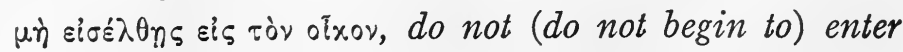
the house.

It will be observed that the first and second examples (present) have reference to the continuance of 
the action, while the third and fourth examples (aorist) have reference to the simple act. In the second example $\mu$ in with the pres. imperative forbids the continuance of the action; while in the fourth example $\mu$ in with the aor. subjunctive forbids the beginning (ingressive) of the action. In the second example the action is going on; in the fourth example the action has not begun. Thus Aktionsart must be considered. In prohibitions to forbid a thing not yet done the aor. subj. (not the imperative) is used with $\mu$ n (see fourth example above).

409. The first aorist act. imperative of $\lambda \dot{v} \omega$ is:
Singular
Plural
2. $\lambda \tilde{u} \sigma \circ \mathrm{V}$
2. $\lambda \cup \operatorname{\sigma \alpha \tau \varepsilon }$
3. $\lambda \cup \sigma \alpha \dot{\tau} \tau \omega$
3. $\lambda \cup \sigma \alpha ́ \tau \omega \sigma \alpha \nu$

a. The origin of -ov of the second pers. sing. is obscure.

Observe that the stem is the aorist stem $\lambda u \sigma \alpha-$.

410. The first aorist middle imperative of $\lambda \dot{\omega} \omega$ is:

\section{Singular}
2. $\lambda \tilde{u} \sigma \alpha \ell$
3. $\lambda \cup \sigma \alpha ́ \sigma \theta \omega$
2. $\lambda \dot{\sigma} \sigma \alpha \sigma \theta \varepsilon$
3. $\lambda u \sigma \alpha ́ \sigma \theta \omega \sigma \alpha \nu$

$a$. The second pers. sing. ending - $\alpha$ probably came from the aorist infinitive. Note accent, $\beta \dot{\alpha} \pi \tau \iota \sigma \alpha$.

411. The aorist passive imperative of $\lambda \dot{\omega} \omega$ is:

Singular

Plural

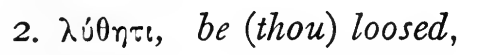

3. $\lambda u \theta \dot{n} \tau \omega$ atc.

2. $\lambda \dot{u} \theta \eta \tau \varepsilon$

3. $\lambda u \theta \dot{\eta} \tau \omega \sigma \alpha \nu$

a. $-\tau$ of the second pers. sing. was $-\theta \imath$. $\theta$ was changed to $\tau$ to avoid the repetition of the rough mute. 
Observe that the forms are made on the aor. pass. stem $\lambda u \theta \varepsilon(\eta)$.

412. In the imperative in Greek there is no first person form. In the first person the subjunctive is used instead of the imperative.

413. The original significance of the imperative was demand or exhortation; but it was not confined to this idea.

The imperative is used in:

I. Commands or exhortations-

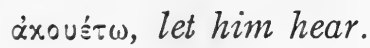

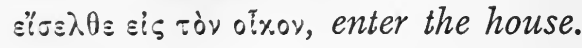

2. Prohibitions-

ù xp'vere quit (don't go on) judging

3. Entreaties-

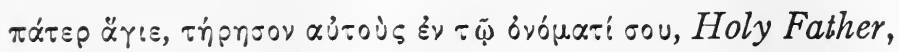
keep them in thy name.

Note.-The negative of the imperative is $\mu$ n.

414.

EXERCISES

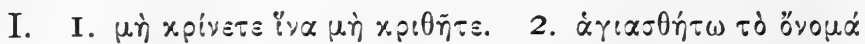

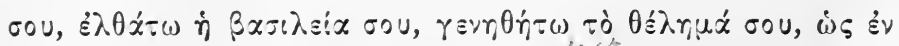

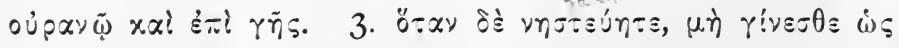

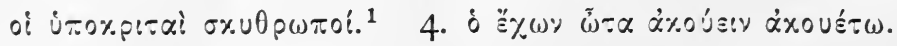

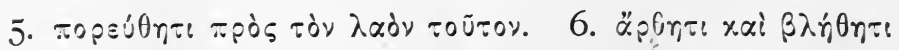

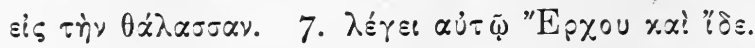

II. I. Let him depart. 2. Guard thyself from the evil one. 3. Say to this people all the words of this life. 4. Quit saying evil things.

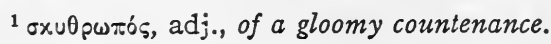




\section{LESSON XLVIII}

\section{Numerals. oủosis}

415.

\section{VOCABULARY}

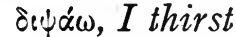

$x \alpha i \ldots x a l$, both-and

$\mu \dot{\prime} \tau \varepsilon \ldots \mu \dot{\prime} \tau \varepsilon$, neither-nor

oủxért, no longer, no more

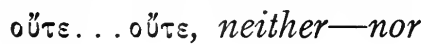

$\pi \alpha^{\prime} \lambda(v$, adv., again

$\pi \varepsilon \iota v \alpha \dot{\omega}$, I hunger, am hun-

gry

$\pi \dot{\omega} \pi 0 \tau \varepsilon$, ever yet

$\tau \hat{~} \ldots$. .x $\alpha^{\prime}$, both-andd

416. Learn the numerals (cardinal and ordinal) from one (first) to twelve (twelfth).

\section{Cardinals}

1. sis, one, etc.

2. Súo

3. $\tau$ peis

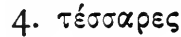

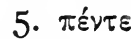

6. $\check{\varepsilon} \xi$

7. غ่ $\pi \tau \dot{\alpha}$

8. $\dot{0} x \tau \dot{\omega}$

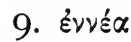

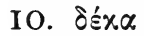

II. $\check{\varepsilon} y \delta \varepsilon x \alpha$

12. $\delta \omega \dot{\delta} \varepsilon \times \alpha$ $\delta \varepsilon x \propto \delta \dot{0} 0$

\section{Ordinals}

$\pi \rho \tilde{\omega} \tau 0 \varsigma$, first, etc.

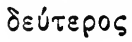

$\tau$ ті́тоs

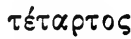

$\pi \dot{\varepsilon} \mu \pi \tau \circ \varsigma$

ह้xт०5

$\ddot{\varepsilon} \beta \delta$ о $\mu \mathrm{s}$

oै $\gamma \delta 005$

हैข $\alpha \tau \circ \varsigma$

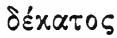

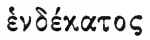

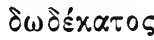

Other numerals may be learned from a lexicon as they are needed.

417. The ordinals have the regular terminations of adjectives of the first and second declensions, as 


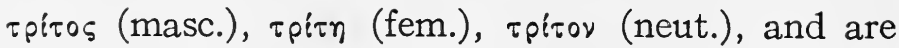
so declined.

418. The cardinals are indeclinable, except the first four and from 200 onward.

The first four are declined as follows:

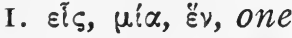
2. Súo, two

Masc., Fem. Neut. Masc., Fem., and Neut.

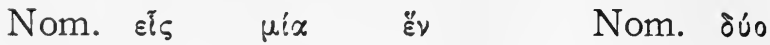
Gen. $\}$ Ebl. Loc. Ins. $\} \dot{\varepsilon} \nu t \quad \mu \iota \tilde{q} \quad \dot{\varepsilon} v t$ Dat. Acc. $\check{\varepsilon} \nu \alpha \quad \mu i \alpha \nu \quad \check{\varepsilon} \nu$

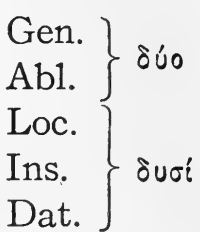

3. $\tau \rho \varepsilon i s, \tau p i \alpha$, three

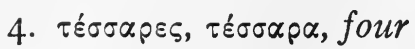
Masc. and Fem. Neut. Masc. and Fem. Neut.

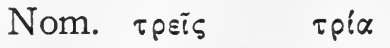

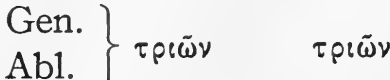
Loc.

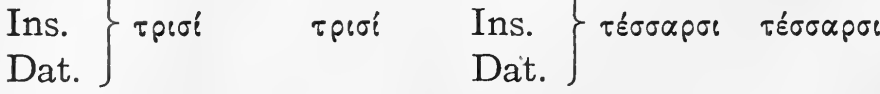

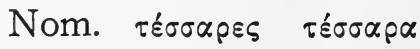
Gen. $\} \tau \varepsilon 1.\} \tau \sigma \sigma \alpha \dot{\rho} \omega \nu \quad \tau \varepsilon \sigma \sigma \alpha \dot{\rho} \omega \nu$ Loc.

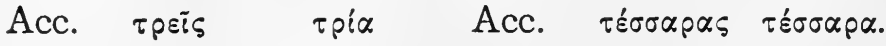

These cardinals agree with the substantives with which they are used.

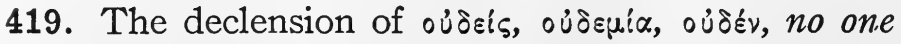
(nobody), nothing, is: 


\section{NEGATIVES}

Masc.

Fem.

Neut.

Nom. oujzís

Gen.

Abl. $\}$ oủsevós

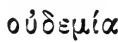

oủరév

Loc.

Ins.

Dat.

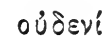

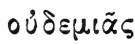

०ủঠevós

Acc.

oủ ริที

○ப̉ $\varepsilon \mu \iota \tilde{\alpha}$

०น์ธะข!́

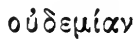

०ưร์้

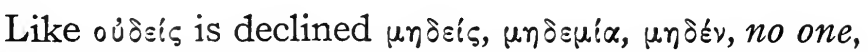
nothing. $\mu \eta \delta \varepsilon i \varsigma$ is generally used wherever $\mu$ 'n would be the appropriate negative.

420. A negative sentence in Greek may have more than one negative particle. In Greek the succession of negatives merely strengthens the first negative if the second (and third) is a compound form like

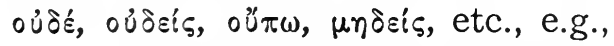

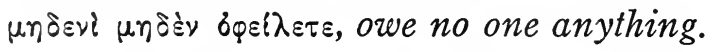

421. ou and $\mu \dot{n}$ are used in direct questions to indicate the kind of answer expected.

I. ou expects the answer yes.

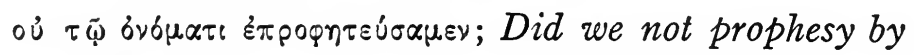
thy name?

2. $\mu$ ' expects the answer no.

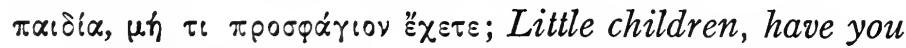
anything to eat? (You haven't anything to eat, have you?)

422. of $\mu \eta n$ is used with the aorist subjunctive (rarely present) or occasionally the future ind. in the sense of an emphatic negative future indicative. 


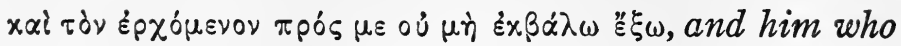
comes to me I will NOT cast out.

423.

EXERCISES

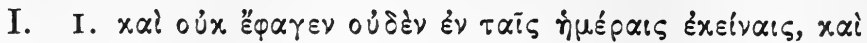

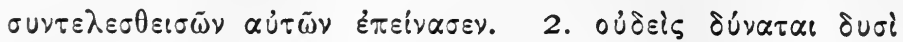

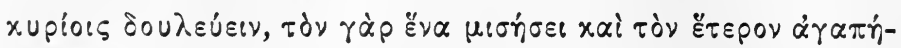

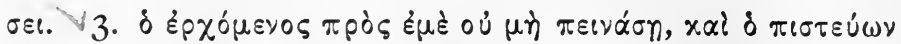

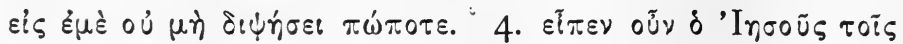

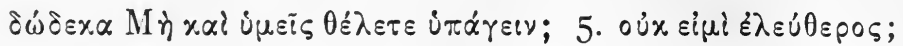

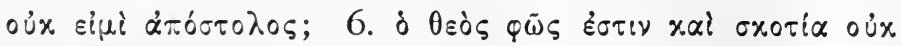

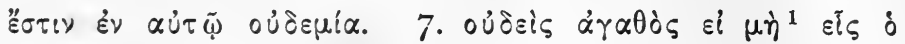

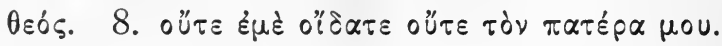

II. I. Let no one enter the house. 2. Did he not see me? 3. I will not serve him. 4. Is he able to serve two masters?

\section{LESSON XIIX}

Present System of Contract Verbs in - $-\varepsilon \omega$.

424.

VOCABULARY

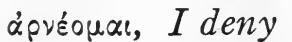

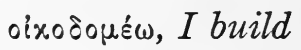

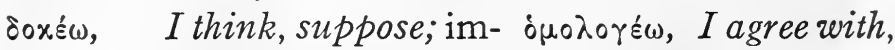
pers. it seems confess

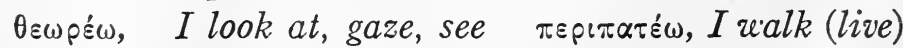
$\mu \varepsilon \tau \alpha \nu \circ \varepsilon \dot{\omega} \omega, I$ repent

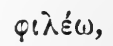

I love

425. The conjugation of verbs with stems in $\alpha, \varepsilon$, or 0 , has been given in all tenses except the present and imperfect. The conjugation of these verbs (stems in $\alpha, \varepsilon$, or 0 ) differs from that of regular $\omega$ - verbs in the present and imperfect tenses only.

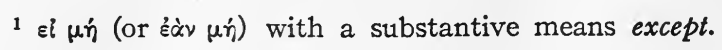


426. In the present and imperfect tenses the vowel $(\alpha, \varepsilon$, or 0$)$ of the stem unites with the thematic vowel (and in some forms the personal ending also) and forms a diphthong or a single long vowel. This is called contraction.

427. The conjugation of $\varphi \iota \lambda \varepsilon \dot{\varepsilon} \omega$ in the present system is as follows:

\section{The present indicative:}

\section{ACTIVE}

Singular
I. $\varphi i \lambda \tilde{\omega}\left(\varphi \iota \lambda \varepsilon^{\prime} \omega\right)$

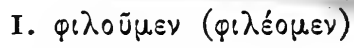
2. $\varphi i \lambda \varepsilon i \varsigma(\varphi \iota \lambda \varepsilon \dot{\varepsilon} \varepsilon \iota \varsigma)$
2. $\varphi(\lambda \varepsilon i \tau \varepsilon(\varphi ! \lambda \varepsilon \varepsilon \varepsilon \tau \varepsilon)$
3. $\varphi i \lambda \varepsilon \tilde{\imath}(\varphi \imath \lambda \varepsilon \varepsilon \varepsilon$ )
3. $\varphi \imath \lambda \circ \tilde{\sigma} \sigma \ell$ ( $\varphi \imath \lambda \varepsilon \dot{\varepsilon} \cup \sigma \iota)$

\section{Middle AND Passive}

Singular

Plural

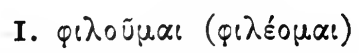

I. $\varphi$ i $\lambda \circ u ́ \mu \varepsilon \theta \alpha \quad(\varphi$ i $\lambda \varepsilon o ́ \mu \varepsilon \theta \alpha)$

2. $\varphi i \lambda \tilde{n}\left(\varphi i \lambda \varepsilon_{n}\right)$

2. $\varphi \iota \lambda \varepsilon \tilde{\imath} \sigma \theta \varepsilon \quad(\varphi \iota \lambda \varepsilon \varepsilon \varepsilon \sigma \theta \varepsilon)$

3. $\varphi i \lambda \varepsilon i \tau \alpha_{l} \quad\left(\varphi l \lambda \varepsilon \varepsilon_{\varepsilon} \tau \alpha_{l}\right)$

3. $\varphi ! \lambda \circ \tilde{u} \nu \tau \alpha_{l}$ ( $\left(\ell \lambda \varepsilon \varepsilon^{\prime} \circ \nu \tau \alpha_{l}\right)$

2. The present subjunctive:

Active

Singular

\section{Plural}

I. $\varphi$ i $\lambda \tilde{\omega}(\varphi เ \lambda \varepsilon \dot{\varepsilon} \omega)$

2. $\varphi i \lambda \tilde{n} s$ ( $\left.\varphi i \lambda \varepsilon_{n} s\right)$

3. $\varphi i \lambda \tilde{n}\left(\varphi i \lambda \varepsilon_{n}\right)$
I. $\varphi \iota \lambda \tilde{\omega} \mu \varepsilon \nu(\varphi \iota \lambda \dot{\varepsilon} \omega \mu \varepsilon \nu)$

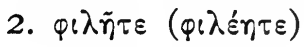

3. $\varphi \imath \lambda \tilde{\omega} \sigma \iota(\varphi \iota \lambda \varepsilon \dot{\omega} \omega \iota)$

Middle AND Passive

\section{Singular}

I. $\varphi \imath \lambda \tilde{\omega} \mu \alpha \imath \quad\left(\phi i \lambda \varepsilon ́ \omega \mu \alpha_{l}\right)$

2. $\left[\varphi \cdot \lambda \tilde{n}\left(\varphi ! \lambda \varepsilon_{n}^{\prime}\right)\right]$

3. $\varphi l \lambda \tilde{\eta} \tau \alpha_{l} \quad\left(\varphi l \lambda \varepsilon^{\prime} \eta \tau \alpha_{l}\right)$

\section{Plural}

I. $\varphi \iota \lambda \omega \mu \varepsilon \theta \alpha \quad(\varphi \iota \lambda \varepsilon \omega \dot{\mu} \mu \varepsilon \theta \alpha)$

2. $\varphi \iota \lambda \tilde{\eta} \sigma \theta \varepsilon(\varphi \iota \lambda \varepsilon \dot{\eta} \sigma \sigma \theta \varepsilon)$

3. $\varphi \ell \lambda \tilde{\omega} \nu \tau \alpha_{l} \quad\left(\varphi l \lambda \varepsilon^{\prime} \omega \nu \tau \alpha_{l}\right)$ 
3. The present imperative:

Active

Singular

2. $\varphi(\lambda \varepsilon !$ ( $\varphi(\lambda \varepsilon \varepsilon)$

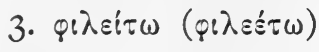

\section{Plural}

2. $\varphi$ i $\lambda \varepsilon i \tau \varepsilon(\varphi \iota \lambda \varepsilon \dot{\varepsilon} \varepsilon \varepsilon)$

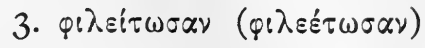

Middle And Passive

Singular

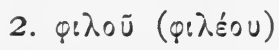

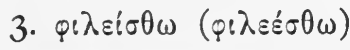

Plural

2. $\varphi i \lambda \varepsilon i \sigma \theta \varepsilon(\varphi i \lambda \varepsilon \varepsilon \varepsilon \sigma \theta \varepsilon)$

3. $\varphi i \lambda \varepsilon i \sigma \theta \omega \sigma \alpha \nu$ ( $\varphi \imath \lambda \varepsilon \varepsilon \varepsilon \sigma \theta \omega \sigma \alpha \nu)$

\section{The present infinitive:}

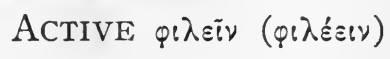

Middle ANd Passive $\varphi \imath \lambda \varepsilon i \sigma \theta \alpha \iota$ ( $\varphi$ i $\lambda \varepsilon \varepsilon \sigma \theta \alpha l$ )

5. The present participle:

Active

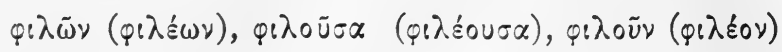

Middle and Passive

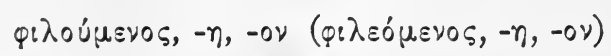

6. The imperfect indicative:

Active

Singular
I. Ėpi

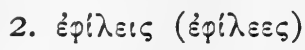

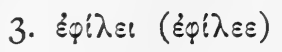

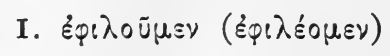

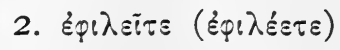
3. Ép!

\section{Middle and Passive}

\section{Plural}

\section{Singular}

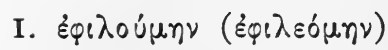
2. E९८

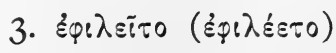

Plural

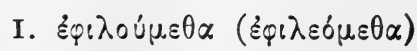
2. $\dot{\varepsilon} \varphi ! \lambda \varepsilon i \sigma \theta \varepsilon(\varepsilon \dot{\varepsilon} \varphi \lambda \varepsilon \varepsilon \varepsilon \sigma \theta \varepsilon)$

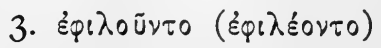


428. The declension of the present active participle $\varphi \iota \lambda \tilde{\omega} \nu,-0 \tilde{u} \sigma \alpha,-\circ \tilde{u} \nu$ is:

Singular

Masc. Fem. Neut.

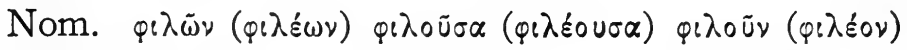

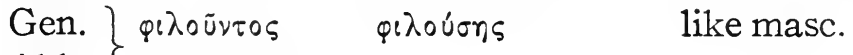

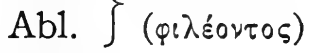

Loc.

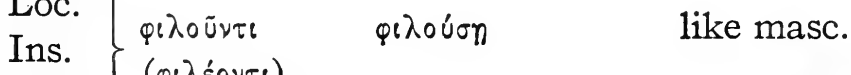

Dat. $\{(\varphi \imath \lambda \varepsilon \dot{0 \nu \tau \tau)}$

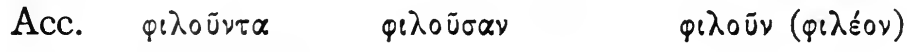

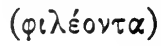

\section{Plural}

Masc.

Fem.

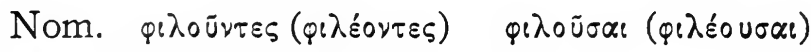

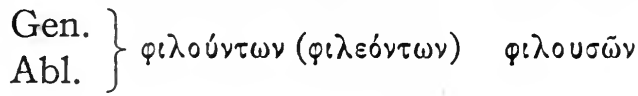

Loc.

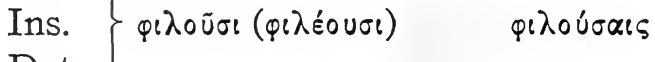

Dat.

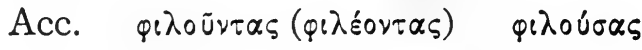

Neut.

Nom. $\varphi$ เ

Gen. $\}$ like masc.

Loc.

Ins. like masc.

Dat.

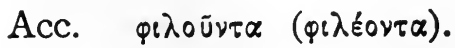


429. The scheme of contraction for regular $-\varepsilon \omega$ verbs is as follows:

$$
\begin{array}{ll}
\varepsilon+\varepsilon=\varepsilon l & \varepsilon+\varepsilon l=\varepsilon l \\
\varepsilon+0=0 u & \varepsilon+\eta=\eta \\
\varepsilon+\omega=\omega & \varepsilon+\circ u=\circ u
\end{array}
$$

430. Observe that: I. The syllable resulting from contraction has an accent if either one of the component syllables had an accent in the uncontracted form. 2. The accent is circumflex, if the first vowel (of the contracting vowels) had the acute; but it is an acute, if the second vowel had the acute.

431.

EXERCISES

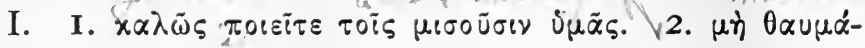

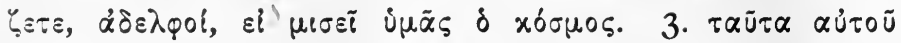

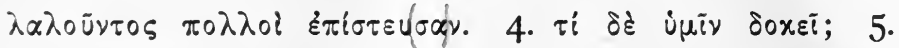

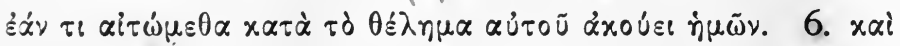

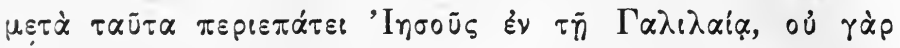

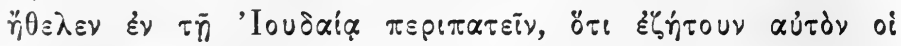

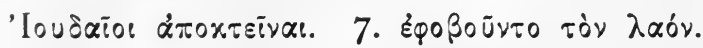

II. I. Who is seeking to kill you? 2. He who hates his brother walks in darkness. 3. Follow me. 4. Quit doing these things. 5. They feared the crowd.

\section{LESSON L}

Comparison of Adjectives and Adverbs

432. VOCABULARY

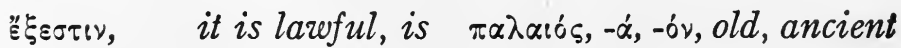
possible $\quad \pi \varepsilon p r \sigma \sigma o ́ s,-\dot{r}_{i},-o v$, abundant

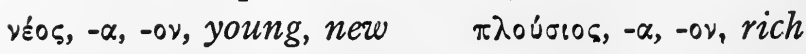


433. The comparative degree of an adjective in -os

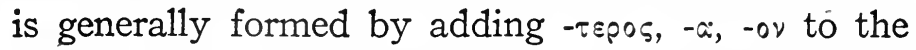
masc. stem of adj. as found in the positive degree. To form the superlative ${ }^{1}$ degree, - $\tau \alpha \tau \circ 5,-\eta$, -ov is added to the masc. stem of adj. as found in the positive degree.

434. Examine carefully the following examples:

Positive Comparative Superlative

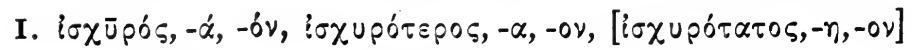

strong stronger strongest

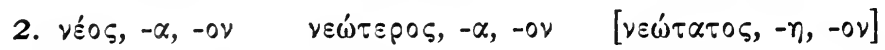

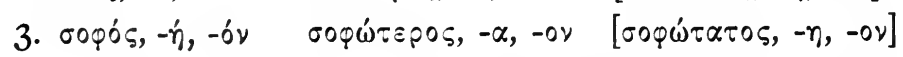

$a$. The forms enclosed in brackets are not found in the New Testament.

Similarly adjectives in - $\varsigma \varsigma$ make the comparison.

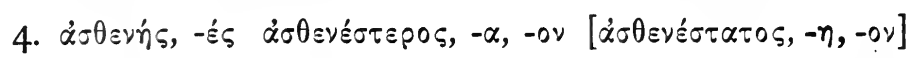

435. Observe: I. If the penult of the adjective (with nom. sing. masc. in -05 ) is short ${ }^{2}$ in the positive, the $\circ$ of the stem is lengthened to $\omega^{3}$ in the comparative and superlative. 2. All comparatives and superlatives have recessive accent.

436. The stem from which the comparative is formed may be an adverb, e.g.,

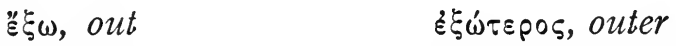

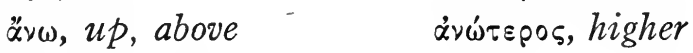

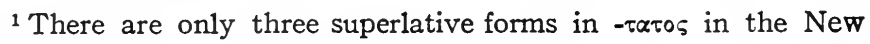
Testament.

2 A penult, although its vowel is short, is considered long if its vowel is followed by two consonants or a double consonant.

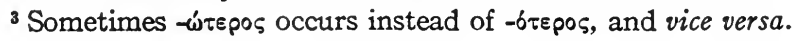


437. With the comparative degree (of adverbs also) the ablative is commonly used to express the standard of comparison, e.g.,

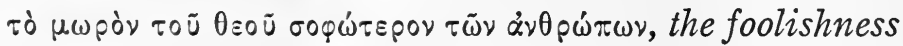
of God (is) wiser than men.

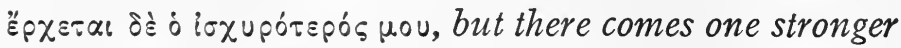
(mightier) than $I$.

438. The comparative may be followed by $\ddot{\eta}$ (than), then the standard of comparison is in the same case as the object compared, e.g.,

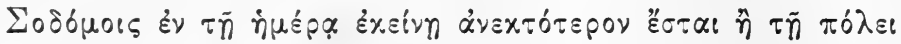
Exxivn, it will be more tolerable in that day for Sodom than for that city.

$a$. $\ddot{\eta}$ is used also in the comparison of clauses.

439. The superlative form is rare in the New Testament. When it occurs, it generally has, not the true superlative sense, but the elative sense of very or exceedingly.

In the New Testament the comparative with the article generally performs the peculiar functions of the superlative, e.g.,

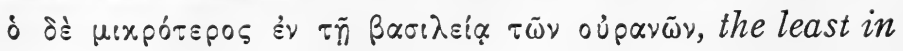
the kingdom of heaven.

440. Adverbs are made from adjectives also. Note carefully the following example.

Positive Comparative Superlative

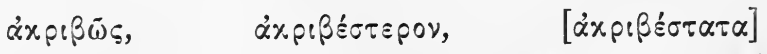
accurately more accurately most accurately adj. axpeßro 
Observe: I. The positive degree of the adverb is made by adding the ablative ending - $\omega 5$ to the adjective stem. (In a mechanical way the positive degree of the adverb is formed from the positive degree of the adjective by changing final $\nu$ of the ablative plural neuter to $s$ and retaining the accent of the adjective.) 2. The comparative of the adverb is the neut. sing. acc. of the adjective. 3. The superlative of the adverb is the neut. plur. acc. of the superlative of the adjective.

441. $\delta \delta \varepsilon, \dot{\eta} \delta \varepsilon$, oi $\delta \varepsilon ́$ are used demonstratively to refer to persons already mentioned in an oblique case, e.g.,

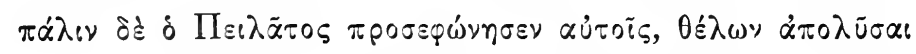

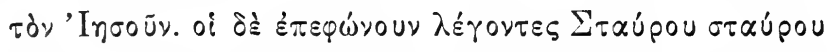
aưróv, And again Pilate spoke to them, wishing to release Jesus. But they shouted, saying, "Crucify, crucify him."

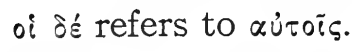

442. In comparisons $\mu \tilde{\alpha} \lambda \lambda \circ \nu$ (more, rather) and $\hat{\eta}$ are used with the positive degree.

443.

EXERCISES

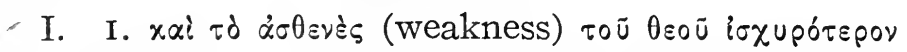

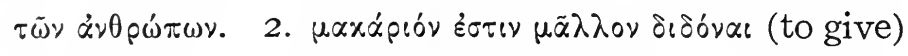

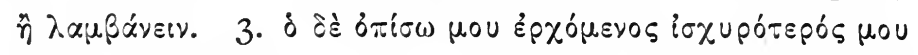

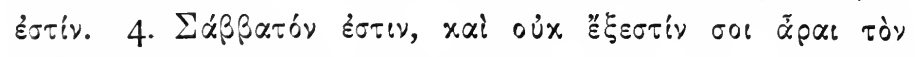

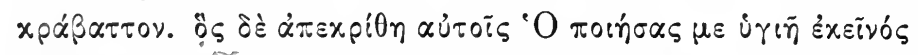

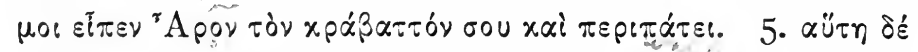

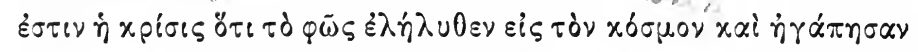

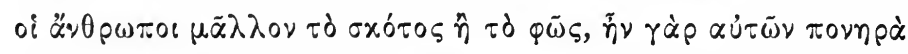

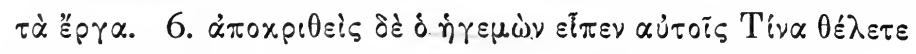

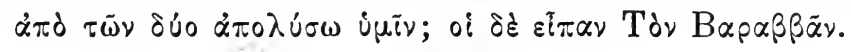


II. I. That man is stronger than I. 2. He said to them, "Come unto me." They said to him, "We are not able to go." 3. The children of God loved light rather $\left(\mu \tilde{\alpha} \lambda \lambda_{0 \nu}\right)$ than darkness. 4. Seek ye rather to enter the kingdom than to die in sin.

\section{LESSON LI}

Comparison of Adjectives and Adverbs (Continued). Declension of Adjectives with Stems in -ov444. VOCABULARY

$\ddot{\alpha} \varphi \rho \omega \nu,-0 \nu$, foolish

$\varepsilon^{r}$, well

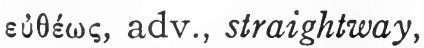
at once

$\tau \hat{\varepsilon} \lambda \varepsilon \iota_{1}$, , $-\alpha,-0 \nu$, finished, complete

445. The following adjectives show irregularities of comparison.

Comparative suffix $-\imath \omega \nu$ (masc.) Superlative suffix - เซros (masc.)

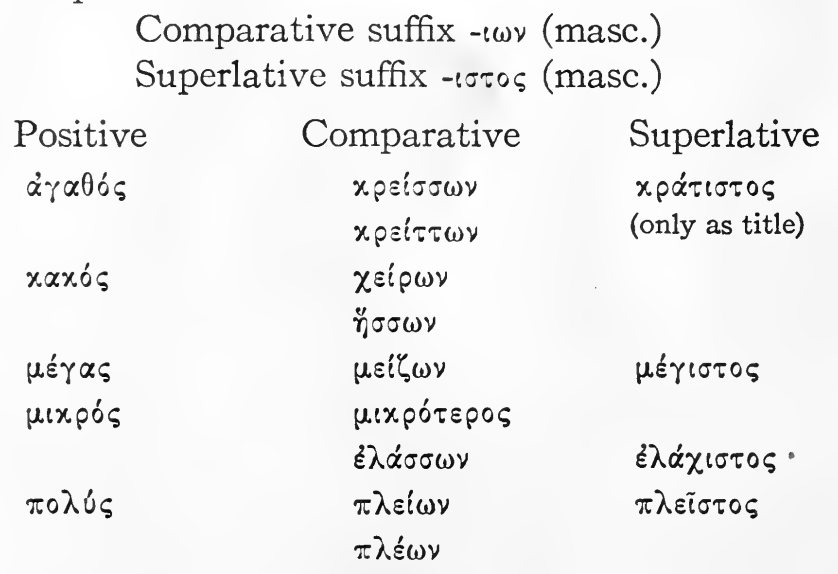

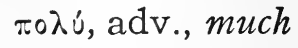
$\tau \alpha \chi \varepsilon \dot{\varepsilon} \omega \varsigma, \tau \alpha \chi \dot{u}$, adv., quickly $\sigma \omega ́ \varphi p \omega \nu,-o v$, of sound mind, sober-minded

$\omega \delta \varepsilon$, adv., here, hither 
446. The declension of $\mu \varepsilon i_{\zeta} \omega \nu,-o \nu$, the comparative of $\mu \varepsilon^{\prime} \gamma \alpha \varsigma$, is:

\section{STEM $\mu \varepsilon i \zeta \circ \nu-(\mu \varepsilon i \zeta 0 \sigma-)$}

Singular

\section{Masc. and Fem.}

Nom. $\mu \varepsilon i \zeta \omega \nu$

Gen.

Abl. $\} \mu s i \zeta$ ovos

Loc.

$\left.\begin{array}{l}\text { Ins. } \\ \text { Dat. }\end{array}\right\} \mu s i \zeta_{\text {ove }}$

Acc. $\mu \varepsilon i \zeta o v \alpha, \mu \varepsilon i \zeta \omega$

\section{Plural}

Masc. and Fem.

Nom. $\mu \varepsilon i \zeta o v \varepsilon s, \mu \varepsilon i \zeta o u s$

Gen.

Abl. $\mu \varepsilon เ \zeta \delta \nu \omega \nu$

Loc.

Ins.

Dat.

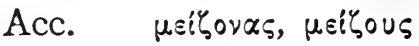

Neut.

$\mu \varepsilon i \zeta{ }_{0}$

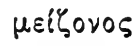

$\mu \varepsilon i \zeta$ ovs

$\mu \varepsilon i \zeta o v$.

\section{Neut.}

$\mu \varepsilon i \zeta \circ \alpha \alpha, \mu \varepsilon i \zeta \omega$

$\mu \varepsilon i \zeta o ́ v \omega \nu$

$\mu \varepsilon i \zeta 0 \sigma$

$\mu \varepsilon i \zeta o v \alpha, \mu \varepsilon i \zeta \omega$.

447. Comparatives in $-(\iota) \omega \nu$ are declined like $\mu s i \zeta \omega \nu$;

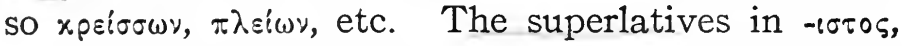
$-\eta,-$ ov are declined like adjectives of the first and second declensions.

448. Adjectives with stems in -ov- are declined like $\mu \varepsilon i \zeta_{\omega \nu}$, except that they do not have the second forms like $\mu \varepsilon i \zeta \omega$ and $\mu \varepsilon i \zeta o u \xi$ : as $\ddot{\alpha} \rho p \omega \nu,-o \nu, \sigma \omega \dot{\rho} \rho \omega \nu,-o \nu$, etc. The voc. sing. of $\ddot{\alpha}_{\varphi} \rho \omega \nu$ is $\ddot{\alpha}_{\varphi \rho} \rho \nu$ (like nom.). 
449. Observe carefully the following examples of the comparison of irregular adverbs. (See $438, \mathrm{I}-3$ ):

Positive

$$
\begin{aligned}
& \varepsilon \tilde{u} \\
& x \alpha \lambda \tilde{\omega} \varsigma \\
& x \alpha \times \tilde{\omega} \varsigma \\
& (\mu \alpha \lambda \alpha) \\
& \pi \circ \lambda \dot{v}
\end{aligned}
$$

हैүYús

$\tau \dot{\alpha} \chi \alpha$ or

$\tau \alpha \chi \varepsilon \omega s$
Comparative Superlative

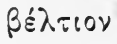

$$
\begin{aligned}
& x \alpha \dot{\lambda} \lambda \text { cov } \\
& \tilde{\eta} \text { бoov } \\
& \mu \tilde{\alpha} \lambda \lambda \text { ov } \\
& \pi \lambda \text { हाо }
\end{aligned}
$$

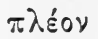

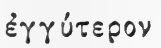

$\tau \alpha \dot{\alpha}$ เov

$\tau \dot{\alpha} \chi \varepsilon\llcorner 0 \nu$ $\mu \alpha \dot{\lambda} 1 \sigma \tau \alpha$

है $\gamma \gamma \operatorname{c\tau \alpha }$

$\tau \dot{\alpha} \chi 1 \sigma \tau \alpha$

$a$. The positive of the adverb is sometimes made from the neut. acc. sing. of the adjective.

\section{EXERCISES}

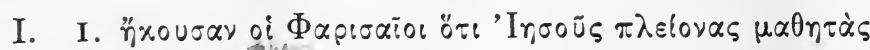

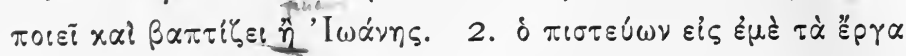

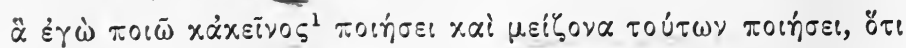

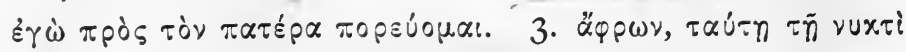

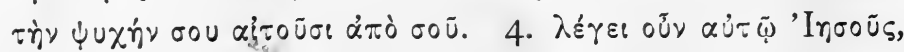

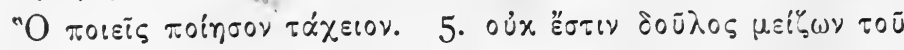

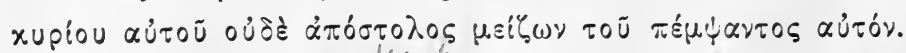

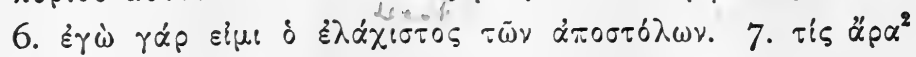

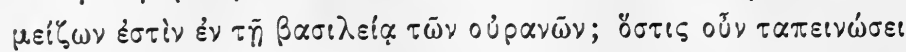

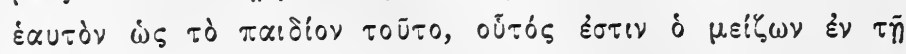
$\beta \alpha \tau \iota \lambda \varepsilon i q u \tau \tilde{\omega} \nu$ ou $\rho \alpha \nu \tilde{\omega} \nu$.

II. I. My brother is greater than I. 2. I hope to come unto you quickly. 3. I am able to do more work than this. 4. Is it lawful to do well on the sabbath?

${ }^{1}$ See $\S 4$, p. $217 . \quad 2 \alpha_{\alpha} \alpha$, an inferential particle, then, therefore. 


\section{LESSON LII}

Present System of Contract Verbs in $-\dot{\alpha} \omega$

451.

VOCABULARY

$\delta\llcorner\alpha \lambda \circ \gamma i \zeta o \mu \alpha$, I consider, reason, $\tau \varepsilon \lambda \varepsilon \cup \tau \alpha \dot{\omega} \omega$, (I finish) discuss

I die

$\varepsilon \dot{\pi} \varepsilon \rho \omega \tau \dot{\alpha} \omega, \quad I$ question, ask (a $\tau \iota \mu \dot{\alpha} \omega, \quad I$ honor question) $\tau 0 \lambda \mu \alpha \dot{\omega} \omega, \quad I$ dare

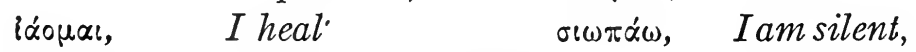
$\pi \lambda \alpha \nu \alpha \dot{\omega}, \quad I$ cause to wander, keep silead astray lence

452. The conjugation of $\gamma \varepsilon \nu \nu \alpha \dot{\omega} \omega$, as an example of the $-\dot{\alpha} \omega$ verbs, in the present system, is:

I. The present indicative:

Active

\section{Singular}

I. $\gamma \varepsilon \nu \nu \bar{\omega}(\gamma \varepsilon \vee \nu \alpha \dot{\omega} \omega)$

2. $\gamma \varepsilon \vee \nu \alpha \tilde{\alpha} \varsigma$ ( $\gamma \varepsilon \vee \nu \alpha \dot{\alpha} \varepsilon i \varsigma)$

3. $\gamma \varepsilon \nu \nu \tilde{\alpha}$ ( $(\gamma \varepsilon \nu \nu \alpha ́ \varepsilon \iota)$

\section{Plural}

I. $\gamma \varepsilon \nu \nu \tilde{\omega} \mu \varepsilon \nu$ ( $\gamma \varepsilon \nu \nu \alpha \dot{O} \mu \varepsilon \nu)$

2. $\gamma \varepsilon \nu \nu \tilde{\alpha} \tau \varepsilon(\gamma \varepsilon \nu \nu \alpha \dot{\varepsilon} \varepsilon \tau)$

3. $\gamma \varepsilon \nu \nu \tilde{\omega \sigma \ell}(\gamma \varepsilon \nu \nu \alpha \dot{\sigma} \cup \sigma \iota)$

\section{Middle AND Passive}

\section{Singular}

I. $\gamma \varepsilon \nu \nu \tilde{\omega} \mu \alpha \ell \quad(\gamma \varepsilon \nu \nu \alpha \dot{0} \mu \alpha \ell)$

2. $\gamma \varepsilon \nu \nu \tilde{\alpha} \sigma \alpha \ell \quad\left(\gamma \varepsilon \nu \nu \alpha_{l} \varepsilon \sigma \alpha l\right)$

3. $\gamma \varepsilon \nu \nu \tilde{\alpha} \tau \alpha \iota \quad\left(\gamma \varepsilon \nu \nu \alpha \dot{\varepsilon} \varepsilon \alpha_{l}\right)$

\section{Plural}

I. $\gamma \varepsilon \nu \nu \omega \dot{\omega} \mu \varepsilon \theta \alpha \quad\left(\gamma \varepsilon \nu \nu \alpha \alpha^{\prime} \mu \varepsilon \theta \alpha\right)$

2. $\gamma \varepsilon \nu \nu \tilde{\alpha} \sigma \theta \varepsilon(\gamma \varepsilon \nu \nu \alpha \dot{\alpha} \varepsilon \sigma \theta)$

3. $\gamma \varepsilon \nu \nu \tilde{\omega} \nu \tau \alpha \ell$ ( $\gamma \varepsilon \nu \nu \alpha ́ o \nu \tau \alpha l)$

2. The present subjunctive:

\section{Active}

\section{Singular}

I. $\gamma \varepsilon \nu \nu \bar{\omega}(\gamma \varepsilon \vee \nu \alpha \dot{\alpha} \omega)$

2. $\gamma \varepsilon \vee \nu \tilde{\alpha} \varsigma$ ( $(\gamma \varepsilon \vee \vee \alpha ́ n \varsigma)$

3. $\gamma \varepsilon \nu \vee \tilde{a}$ ( $(\gamma \varepsilon \nu \vee \alpha ́ n)$

\section{Plural}

I. $\gamma \varepsilon \nu \nu \tilde{\omega} \mu \varepsilon \nu(\gamma \varepsilon \nu \nu \dot{\alpha} \omega \mu \varepsilon \nu)$

2. $\gamma \varepsilon \nu \nu \tilde{\alpha} \tau \varepsilon(\gamma \varepsilon \nu \nu \alpha \dot{\eta} \tau \varepsilon)$

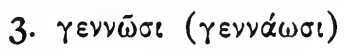




\section{BEGINNER'S GREEK GRAMMAR}

Note that the contract forms of the indicative and subjunctive active are alike.

\section{Middle and Passive}

\section{Singular}

I. $\gamma \varepsilon v \nu \bar{\omega} \mu \alpha$ l $\left(\gamma \varepsilon \nu \nu \alpha \dot{\omega} \omega \alpha_{l}\right)$

2. $\left[\gamma \varepsilon \nu \vee \tilde{\alpha}\left(\gamma \varepsilon \vee \nu \alpha^{\prime} \eta\right)\right]$

3. $\gamma \varepsilon \nu \nu \bar{\alpha} \tau \alpha_{l}\left(\gamma \varepsilon \nu \nu \alpha_{\eta} \tau \alpha_{l}\right)$

\section{Plural}

I. $\gamma \varepsilon \nu \nu \omega \dot{\mu} \mu \varepsilon \theta \alpha \quad(\gamma \varepsilon \nu \nu \alpha \dot{\omega} \mu \varepsilon \theta \alpha)$

2. $\gamma \varepsilon \nu \nu \bar{\alpha} \sigma \theta \varepsilon(\gamma \varepsilon \nu \nu \alpha ́ \eta \sigma \theta \varepsilon)$

3. $\gamma \varepsilon \nu \nu \omega \tilde{\nu \tau \alpha i}\left(\gamma \varepsilon \nu \nu \alpha ́ \omega \nu \tau \alpha_{i}\right)$

3. The present imperative:

Active

Singular

2. $\gamma \varepsilon \hat{\varepsilon} \nu \alpha \alpha(\gamma \varepsilon \dot{\varepsilon} \nu \alpha \varepsilon)$

3. $\gamma \varepsilon \nu \nu \alpha \dot{\tau} \tau \omega(\gamma \varepsilon \nu \nu \alpha \varepsilon \dot{\tau} \omega)$
Plural

2. $\gamma \varepsilon \nu \nu \tilde{\alpha} \tau \varepsilon \quad(\gamma \varepsilon \nu \nu \alpha \dot{\varepsilon} \varepsilon \varepsilon)$

3. $\gamma \varepsilon \nu \nu \alpha \dot{\alpha} \tau \omega \sigma \alpha \nu(\gamma \varepsilon \nu \nu \alpha \varepsilon \dot{\tau} \tau \omega \sigma \alpha \nu)$

Middle ANd Passive

\section{Singular}

2. $\gamma \varepsilon \nu \nu \bar{\omega}(\gamma \varepsilon \nu \nu \alpha \dot{\alpha} 0 u)$

3. $\gamma \varepsilon \nu \vee \alpha \dot{\sigma} \sigma \theta \omega(\gamma \varepsilon \nu \nu \alpha \varepsilon \sigma \theta \omega)$
Plural

2. $\gamma \varepsilon \nu \nu \bar{\alpha} \sigma \theta \varepsilon \quad(\gamma \varepsilon \nu \nu \alpha \dot{\varepsilon} \varepsilon \theta \varepsilon)$

3. $\gamma \varepsilon \nu \nu \alpha \dot{\sigma} \sigma \omega \sigma \alpha \nu(\gamma \varepsilon \nu \nu \alpha \varepsilon \varepsilon \sigma \theta \omega \sigma \alpha \nu)$

4. The present infinitive:

Active

$\gamma \varepsilon \nu \nu \bar{\alpha} \nu$ ( $\gamma \varepsilon \nu \nu \alpha \dot{\varepsilon} \varepsilon \nu)$; some editors write $\gamma \varepsilon \nu \nu \alpha \underline{\alpha} \nu$

Note. $\gamma \varepsilon \nu \nu \bar{\alpha} \nu$ really represents $\gamma \varepsilon \nu \nu \alpha \varepsilon \varepsilon \nu$, for the inf. ending $-\varepsilon \iota \nu$ is a contraction of the thematic vowel $\varepsilon$ and $\varepsilon \nu$.

\section{Middle and Passive}

$\gamma \varepsilon \varepsilon \nu \bar{\alpha} \sigma \theta \alpha l \quad(\gamma \varepsilon \nu \nu \alpha \dot{\varepsilon} \varepsilon \sigma \theta \alpha l)$

5. The present participle:

ACtive

$\gamma \varepsilon \nu \nu \tilde{\omega} \nu$ ( $\gamma \varepsilon \nu \nu \alpha \dot{\omega} \omega \nu), \quad \gamma \varepsilon \nu \nu \tilde{\omega} \sigma \alpha(\gamma \varepsilon \nu \nu \alpha \dot{\alpha} \sigma \sigma \alpha), \quad \gamma \varepsilon \nu \nu \tilde{\omega} \nu$ ( $\gamma \varepsilon \nu \nu \alpha \dot{\alpha} \sigma \nu)$

Middle and Passive

$\gamma \varepsilon \nu \nu \dot{\mu} \mu \varepsilon \nu \circ \varsigma,-\eta,-O \nu$ ( $\left.\gamma \varepsilon \nu \nu \alpha \sigma^{\prime} u \varepsilon \nu \circ \varsigma\right)$ 
6. The imperfect indicative:

\section{Active}

Singular

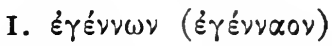

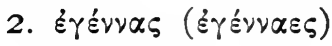

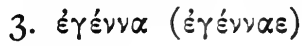

\section{Plural}
I. $\varepsilon \gamma \varepsilon \nu \nu \tilde{\omega} \mu \varepsilon \nu(\xi \dot{\varepsilon} \gamma \varepsilon \nu \nu \alpha \dot{\alpha} 0 \mu \varepsilon \nu)$
2. $\varepsilon \gamma \varepsilon \nu \nu \tilde{\alpha} \tau \varepsilon(\varepsilon \xi \varepsilon \nu \nu \alpha \dot{\varepsilon} \varepsilon \varepsilon)$

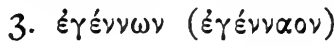

Note. In the third plur. a form like É $\varepsilon^{\prime}$ rvouv is sometimes found. Thus from $\dot{\varepsilon} \rho \omega \tau \dot{\alpha} \omega$, imperfect

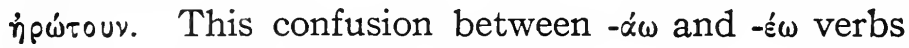
began early in the Ionic.

\section{Middle and Passive}

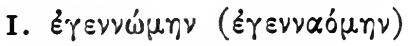
I. $\varepsilon \dot{\gamma} \varepsilon \nu \nu \dot{\omega} \mu \varepsilon \theta \alpha\left(\varepsilon \dot{\varepsilon} \gamma \varepsilon \nu \nu \alpha o^{\prime} \mu \varepsilon \theta \alpha\right)$
2. Еं

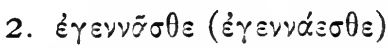

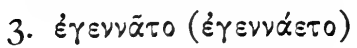

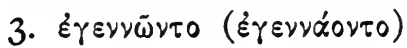

453. The declension of the present active participle $\gamma \varepsilon \nu \nu \tilde{\omega} \nu,-\tilde{\omega} \sigma \alpha, \tilde{\omega} \nu$ is:

\section{Singular}

\section{Masc.}

Nom. $\quad \gamma \varepsilon \nu \nu \tilde{\omega} \nu$ ( $\gamma \varepsilon \nu \nu \alpha \dot{\omega} \omega \nu)$

Gen.

Abl.

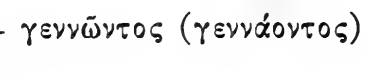

Loc.

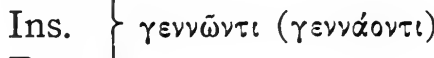
Dat.

Acc. $\quad \gamma \varepsilon \nu \nu \tilde{\omega} \nu \tau \alpha(\gamma \varepsilon \nu \nu \alpha \dot{\alpha} \sigma \tau \alpha)$

Fem.

$\gamma \varepsilon \nu \nu \tilde{\omega} \sigma \alpha \quad(\gamma \varepsilon \nu \nu \alpha \dot{\alpha} \circ \cup \sigma \alpha)$

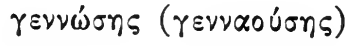

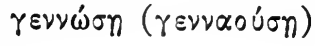

$\gamma \varepsilon \nu \nu \bar{\omega} \sigma \alpha \nu(\gamma \varepsilon \nu \nu \alpha \dot{\alpha} 0 v \sigma \alpha \nu)$

Neut.

Nom. $\quad \gamma \varepsilon \nu \nu \tilde{\omega} \nu$ ( $\gamma \varepsilon \nu \nu \alpha \dot{o \nu})$

Gen. $\mathrm{Abl}$. $\}$ like masc. 


\section{BEGINNER'S GREEK GRAMMAR}

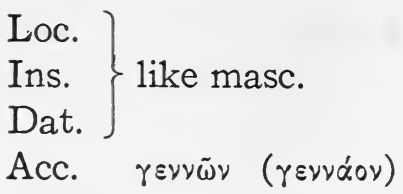

\section{Plural}

Masc. Fem.

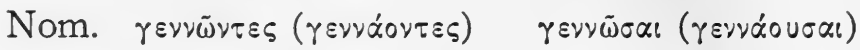

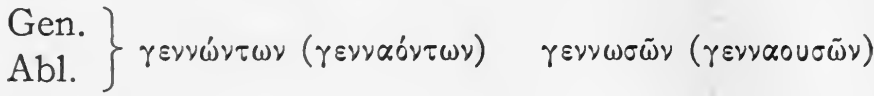

Loc.

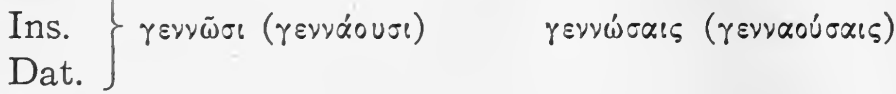

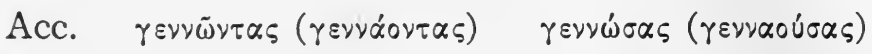

Neut.

Nom. $\quad \gamma \varepsilon \nu \nu \tilde{\omega} \nu \tau \alpha$ ( $\gamma \varepsilon \nu \nu \alpha \dot{0} о \tau \alpha)$

Gen. $\}$ like masc.

Loc.

Ins. like masc.

Dat.

Acc. $\quad \gamma \varepsilon \vee \nu \tilde{\omega} \nu \tau \alpha(\gamma \varepsilon \vee \nu \alpha \dot{0} \vee \tau \alpha)$

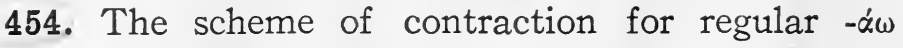
verbs is as follows:

$$
\begin{aligned}
& \alpha+\varepsilon=\alpha \\
& \alpha+\eta=\alpha \\
& \alpha+\varepsilon \imath=\alpha \\
& \alpha+\varepsilon \imath(=\varepsilon+\varepsilon)=\alpha \\
& \alpha+\eta=\alpha
\end{aligned}
$$

$\alpha+0=\omega$

$\alpha+\omega=\omega$

$\alpha+\circ v=\omega$ (since ov in these uncontracted forms is a spurious diphthong, i.e., u is not present in the uncontracted form of ou).

For the accent see 430 . 
455.

\section{EXERCISES}

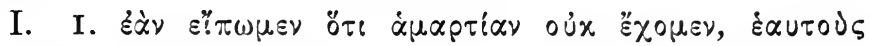

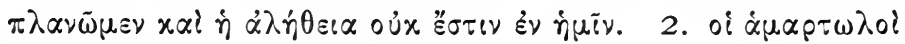

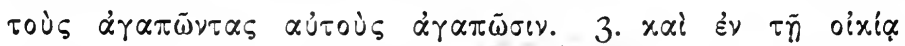

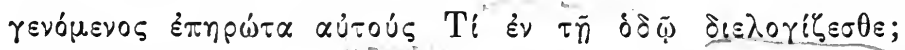

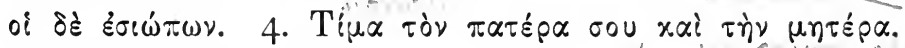

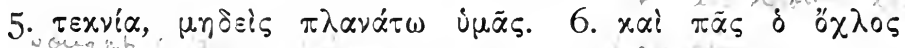

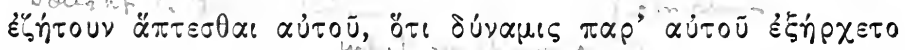

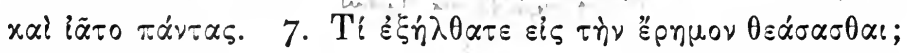

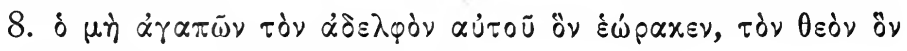

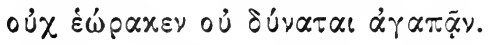

II. I. Let us love one another. 2. He who loves his brother keeps the commandment of God. 3. The disciples were not able to heal him. 4. They were asking him concerning the kingdom.

\section{LESSON LIII}

Impersonal Verbs. $\pi \rho^{i \nu}(\ddot{\eta})$ and the Infinitive.

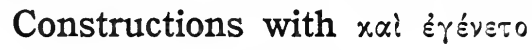

456.

VOCABULARY

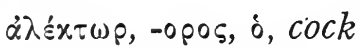

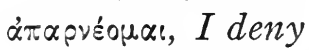

$\delta$ เaxové(), I serve, minister

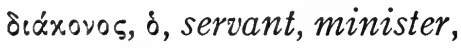

deacon

yoivós, -ńn, -b̀v, common, un-

xoเvów, I make common, unclean

$\mu \alpha \nu \theta \dot{\alpha} \nu \omega$, I learn; second aor. "ै $\mu \alpha \theta 0 \nu$

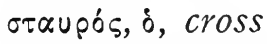

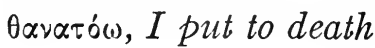

clean

457. There are some verbs used in the third person singular with an impersonal subject, called impersonal verbs. Examine the following examples: 


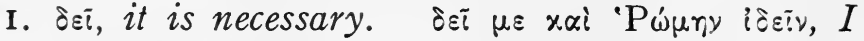
must see Rome also (it is necessary for me to see Rome also). $\mu \varepsilon$ is the acc. of general reference with

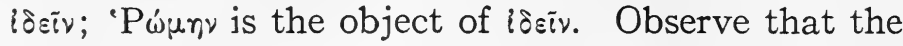
subject of $\delta \varepsilon i$ is $i \delta \varepsilon i \nu$.

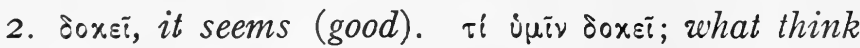
you? (What seems (good) to you?) Observe the case of $\dot{u} \mu i v . \quad \delta \circ x \varepsilon^{\omega} \omega$ is used in the personal construction also.

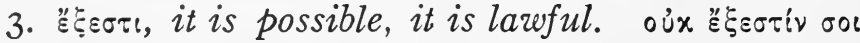

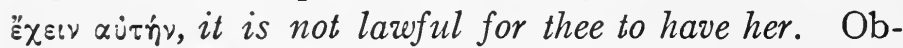

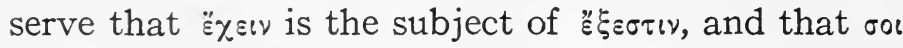
is in the dative case.

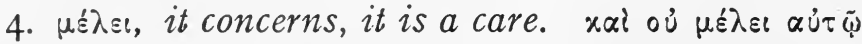
$\pi \varepsilon \rho i \tau \bar{\omega} \nu \pi \rho \circ \beta \alpha$ ' $\omega \nu$, he cares not for the sheep (it is not a care to him concerning the sheep).

458. $\pi$ piv (or $\pi$ piv $\ddot{\eta}$ ), before, is frequently used with the infinitive (in the ablative case). E.g.,

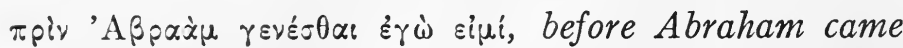
into being, I am.

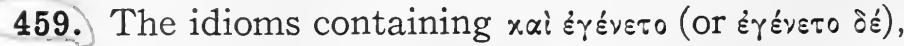
and it came to pass (and it happened), are so common in the New Testament that they call for a special note. The New Testament has four constructions

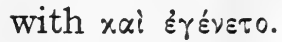

I. $x \alpha i$ ย่

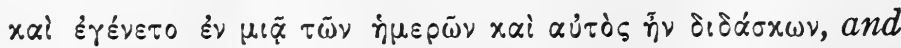
it came to pass, on one of the days, that he was teaching.

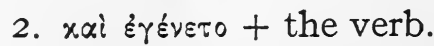

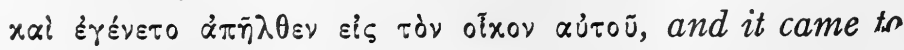
pass that he departed to his home. 


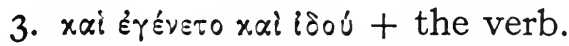

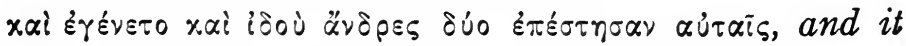
came to pass that, behold, two men stood by them.

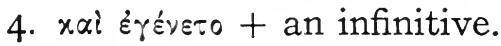

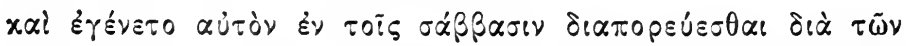

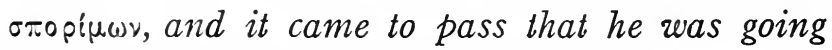
through the grain-fields on the Sabbath.

460.

EXERCISES

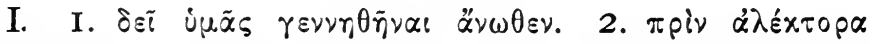

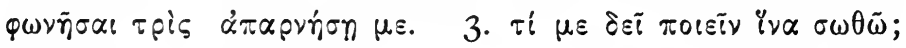

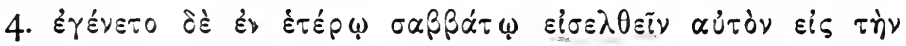

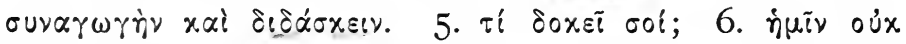

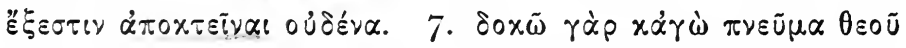

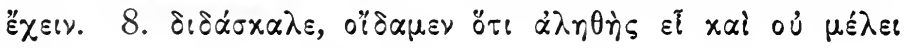
oor $\pi \varepsilon p i$ oủesvós.

II. I. It is necessary to go into the house. 2. He does not care for any one. 3. It came to pass before he went into the house that his brothers came to him. 4. It is not lawful for a man to kill any one.

\section{LESSON LIV}

\section{Present System of Contract Verbs in $-6 \omega$}

461.

VOCABULARY

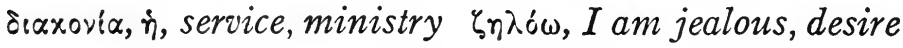
$\delta เ x a\llcorner o \omega$, I declare righteous, eagerly

justify

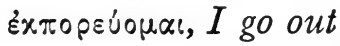

$\varepsilon \dot{\varepsilon} \omega \dot{\pi} \leftarrow \circ \nu$, prep. with gen., before, in presence of

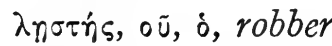

ouoców, I make like

$\pi \rho \circ \varphi \eta \tau \varepsilon \dot{\omega} \omega, I$ prophesy 
462. The conjugation of $\pi \lambda \eta p o ́ \omega$, as an example of the -ow verbs, in the present system, is:

I. The present indicative:

Singular

I. $\pi \lambda \eta p \tilde{\omega}(\pi \lambda \eta \rho \hat{\omega} \omega)$

2. $\pi \lambda \eta$ poís $(\pi \lambda \eta \eta p \delta \varepsilon เ \varsigma)$

3. $\pi \lambda \eta p \circ \tilde{i}(\pi \lambda \eta p o ́ \varepsilon \iota)$
ACTIVE

\section{Plutral}

I. $\pi \lambda \eta p \circ \tilde{\mu} \mu \varepsilon \nu(\pi \lambda \eta p o ́ o \mu \varepsilon \nu)$

2. $\pi \lambda \eta p \circ о \tilde{\tau \varepsilon}(\pi \lambda \eta p o ́ \varepsilon \tau \varepsilon)$

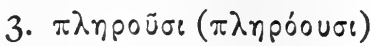

\section{Middle and Passive}

\section{Singular}

I. $\pi \lambda \eta p \circ \tilde{\mu} \mu \alpha \iota(\pi \lambda \eta p \rho \dot{o} \mu \alpha !)$

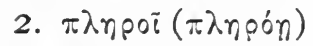

3. $\pi \lambda \eta p \circ \tilde{u} \tau \alpha_{l}\left(\pi \lambda \eta p \rho ́ \varepsilon \tau \alpha_{l}\right)$

\section{Plural}

I. $\pi \lambda \eta \rho \circ u ́ \mu \varepsilon \theta \alpha(\pi \lambda \eta \rho \circ \delta \mu \varepsilon \theta \alpha)$

2. $\pi \lambda \eta p \circ \tilde{u} \sigma \theta \varepsilon(\pi \lambda \eta p \rho \dot{\varepsilon} \varepsilon \theta \varepsilon)$

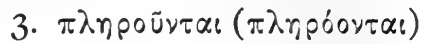

2. The present subjunctive:

\section{Active}

Singular

I. $\pi \lambda \eta \rho \tilde{\omega}(\pi \lambda \eta \rho \dot{\omega} \omega)$

2. $\pi \lambda \eta p o \tilde{s}(\pi \lambda \eta p o ́ n s)$

3. $\pi \lambda$ проі ( $\pi \lambda$ npón)
Plural

I. Like the present

3. $\}$ indicative (probably)

The plural of the present subjunctive active of -ow verbs in New Testament seems to be like the pres. indic. Note that the pres. indic. and subj. active are alike. The pres. subj. act. was probably assimilated to the pres. ind. act. There is doubt concerning the plur. forms that occur in the New Testament.

\section{Middle ANd Passive}

\section{Singular}

I. $\pi \lambda \eta p \tilde{\omega} \mu \alpha \iota(\pi \lambda \eta p o ́ \omega \mu \alpha \iota)$

2. $[\pi \lambda \eta p \circ i[(\pi \lambda \eta p o ́ n)]$

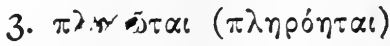

Plural

The plural does not occur in the New Testament 
If the plural had been used in the New Testament it would have probably been like the pres. ind.

Forms in brackets [] are not found in the New Testament.

3. The present imperative:

\section{Active}

Singular

2. $\pi \lambda \dot{n} \rho \circ u(\pi \lambda \dot{n} \rho \circ \varepsilon)$

3. $\pi \lambda \eta p \circ u ́ \tau \omega(\pi \lambda \eta p \circ \varepsilon \dot{\tau} \omega)$

\section{Plural}

2. $\pi \lambda \eta p \circ \tilde{\tau} \tau \varepsilon(\pi \lambda \eta \rho \dot{\sigma} \varepsilon \tau \varepsilon)$

3. $\pi \lambda \eta p \circ u ́ \tau \omega \sigma \alpha \nu(\pi \lambda \eta \rho \circ \varepsilon ́ \tau \omega \sigma \alpha \nu)$

\section{Middle and Passive}

\section{Singular}

\section{Plural}
2. $\pi \lambda \eta p \circ о \tilde{u}$ ( $\pi \lambda \eta$ póou)
2. $\pi \lambda \eta \rho \circ \tilde{\sigma} \sigma \theta \varepsilon(\pi \lambda \eta \rho \sigma ́ \varepsilon \sigma \theta \varepsilon)$

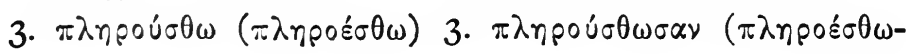 $\sigma \alpha \nu)$

4. The present infinitive:

\section{Active}

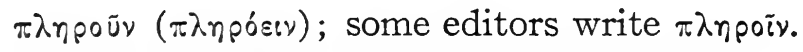

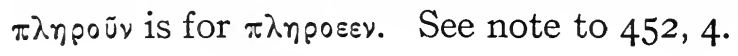

\section{Middle And Passive}

$\pi \lambda \eta p \circ \tilde{u} \sigma \theta \alpha \ell \quad(\pi \lambda \eta p \rho ́ \varepsilon \sigma \theta \alpha l)$.

5. The present participle:

\section{Active}

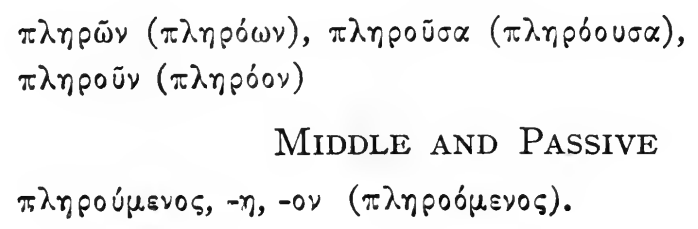




\section{4}

\section{BEGINNER'S GREEK GRAMMAR}

6. The imperfect indicative:

\section{ACTIVE}

Singular

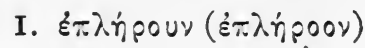

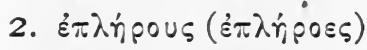

3. $\dot{\varepsilon} \pi \lambda \dot{r}, \rho \circ u(\xi \pi \lambda \dot{r}, \rho \circ)$

\section{Plural}

I. $\dot{\varepsilon} \pi \lambda \eta p \circ \tilde{u} \mu \varepsilon \nu\left(\dot{\varepsilon} \pi \lambda_{1} \rho \dot{0} \circ \mu \varepsilon \nu\right)$

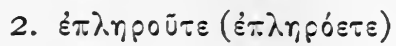

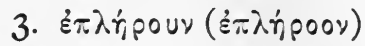

In the third plur. a form like $\varepsilon \pi \lambda \eta p \circ \tilde{u} \sigma \alpha \nu$ ( $\dot{\varepsilon} \pi \lambda \eta p o ́ \sigma \alpha \nu$ ) is found.

\section{Middle and Passive}

\section{Singular}

Plural

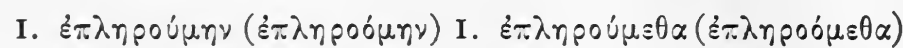

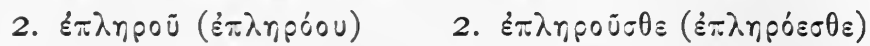

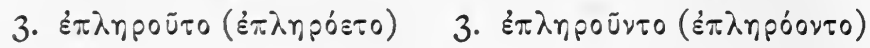

463. The present active participle $\pi \lambda_{\eta} \rho \tilde{\omega} \nu, \pi \lambda \eta \rho \circ \tilde{\sigma} \sigma \alpha$,

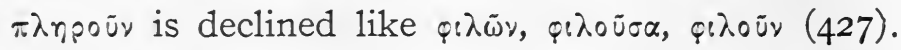
The result of contraction is the same in both cases: $\varepsilon+0=0 u$; and $\circ+0=0 u$.

464. The scheme of contraction for regular -ow verbs is as follows:

$$
\begin{array}{lll}
0+\varepsilon=0 u & 0+\omega=\omega & 0+\circ u=0 u \\
0+o=0 u & 0+\varepsilon l=0 ! & \\
0+\eta=\omega & 0+\eta=0 ! &
\end{array}
$$

465.

\section{EXERCISES}

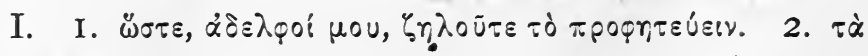

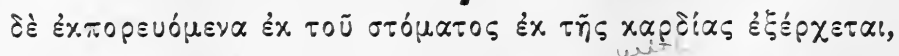

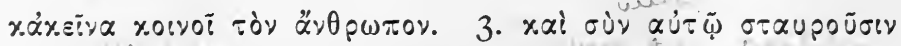

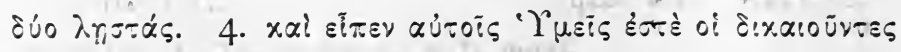

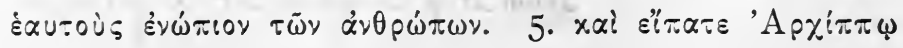

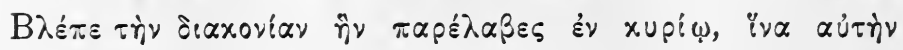




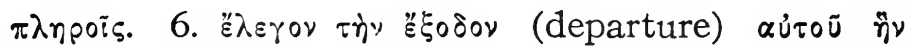

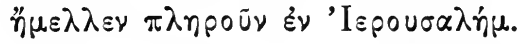

II. I. They were crucifying him with two robbers. 2. Let us love him who justifies us. 3. The works of the righteous are being made manifest. 4. That which goes into the mouth does not defile man.

\section{LESSON LV}

Conjugation of $\mu$-verbs: $\delta i \delta \omega \mu$. Second Aorist of $\gamma \leftarrow \nu \omega \dot{\sigma x \omega}$

466.

VOCABULARY

$\delta i \delta \omega \mu \iota, I$ give, deliver

$\dot{\alpha} \pi 0 \delta i \delta \omega \mu$, I give up or back; restore; pay; midd., sell

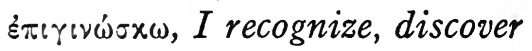

$\varepsilon \pi \iota \delta i \delta \omega \mu, I$ give over

$\pi \alpha \rho \alpha \delta i \delta \omega \mu, I$ give over (to another), deliver up, betray

467. Greek verbs are of two main conjugations, the $\omega$-conjugation and the $\mu$-conjugation. The conjugation which has been studied thus far, except si $\mu$, is the $\omega$-conjugation ( $\omega$-verbs). The verbs in $-\omega$ are by far more common than the verbs in $-\mu$. The verbs (or conjugations) are so named because the ending of the first person singular present indicative active of one is $-\omega$ and of the other is $-\mu$.

468. $\mu$-verbs differ from $\omega$-verbs only in the present and second aorist (called $\mu$-aorist) systems. The essential difference between the $\mu$-verbs and $\omega$-verbs in these systems is that the $\mu$-verbs do not have the thematic vowel $\% / \epsilon$ which the $\omega$ - 
verbs have, before the personal endings. The subjunctive of the $\mu$-verbs, however, has the thematic vowel $\omega / \eta$ (mode-sign). In the other tense systems the $\mu_{\imath}$-verbs and the $\omega$-verbs are conjugated alike.

469. The principal parts of $\delta i \delta \omega \mu{ }^{1}$ are:

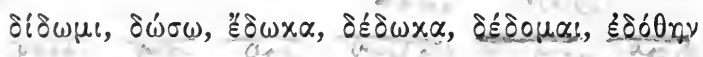

Observe that: I. The verb stem is $\delta 0-$ 2. The present stem is the reduplicated verb-stem, with the vowel $\mathrm{s}$ in the reduplication.

470. The present active of $\delta i \delta \omega \mu$ is:

I. Indicative:

\section{Singular}

I. $\delta i \delta \omega \mu \mathrm{s}$

2. $\delta 1 \delta \omega s$

3. $\delta i \delta \omega \sigma t$
Plural
I. $[\delta i \delta \circ \mu \varepsilon \nu]$
2. $[\delta 1 \delta 0 \tau \varepsilon]$
3. $\delta เ \delta \delta \alpha \sigma t$

In the first sing. a form $\delta, \delta \tilde{\omega}$ (from $\delta 1 \delta \delta \omega$ ) occurs,

2. Subjunctive:

\section{Singular}
I. $[\delta, \delta \tilde{\omega}]$
2. $[\delta ! \delta \tilde{\varphi} \varsigma$ or $\delta, \delta 0 i \varsigma]$
3. $\delta: \delta \bar{\varphi}$ or $\delta ! \delta 0 i$

3. Imperative:

Singular
2. $\delta i \delta 0 u$
3. $\delta เ \delta o ́ \tau \omega$

Plural
I. $[\delta เ \delta \tilde{\omega} \mu \varepsilon \nu]$
2. $[\delta(\delta \omega \tau \varepsilon]$
3. $[\delta เ \delta \tilde{\omega} \sigma t]$

4. Infinitive:

\section{Plural}

2. $\delta i \delta o \tau \varepsilon$

3. $[\delta เ \delta \delta \tau \omega \sigma \alpha \nu]$

\section{$\delta เ \delta \dot{v} v a t$}

${ }^{1} \mu$, -verbs compounded with prepositions are numerous. The uncompounded forms of all $\mu \cdot$-verbs are given as quotable in the New Testament, although the form may be found only in compounds. 
5. Participle:

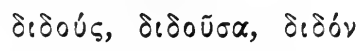

The participle is declined like $\lambda u ́ \omega \nu$ except for the nom. sing. masc., and the accent.

471. The imperfect indicative active of $\delta i \delta \omega \mu$ is:

Singular

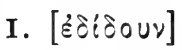
I. $[\varepsilon \dot{\delta} \delta(\delta \circ \mu \varepsilon \nu]$

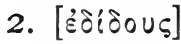

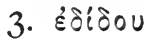

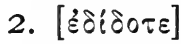

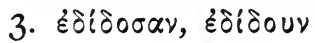

Plural

472. The present middle and passive of $\delta i \delta \omega \mu$ is:

I. Indicative:
Singular
Plural
I. $[\delta i \delta o \mu \alpha l]$
I. $\delta เ \delta \delta \mu \varepsilon \theta \alpha$
2. $[\delta(\delta 0 \sigma \alpha t]$
2. $[\delta 1 \delta 0 \sigma \theta \varepsilon]$
3. $\delta i \delta 0 \tau \alpha t$
3. $[\delta ! \delta 0 \nu \tau \alpha i]$

2. Subjunctive:

Does not occur in the New Testament.

3. Imperative:

Does not occur in the New Testament.

4. Infinitive:

$$
\delta i \delta 0 \sigma \theta a \imath
$$

5. Participle:

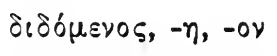

473. The imperfect indicative middle and passive of $\delta i \delta \omega \mu$ is:

Singular

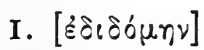

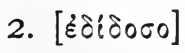

3. $\varepsilon \delta \hat{\delta} \delta 0 \tau 0, \varepsilon \delta \delta i \delta \varepsilon \tau 0$
Plural

I. $\left[\varepsilon \delta \delta_{\iota} \delta \dot{\rho} \mu \theta \alpha\right]$

2. $[\varepsilon \delta(\delta \circ \sigma \theta \varepsilon]$

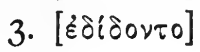


474. The aorist ( $\mu$-aorist) active of $\delta i \delta \omega \mu$ is:

I. Indicative:

\section{Singular}
I. $\varepsilon \delta \omega x \alpha$
2. $\check{\varepsilon} \delta \omega x \alpha \varsigma$
3. $\varepsilon \delta \omega x \varepsilon$

Plural

I. $\varepsilon \delta \dot{\delta} x \alpha \mu \varepsilon \nu$

2. $\varepsilon \delta \dot{\omega} x \alpha \tau \varepsilon$

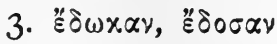

Aorists made with the suffix $-x \alpha$ are called $x$ aorists. Actually they are not $\mu$ - aorists.

2. Subjunctive:
Singular
Plural
I. $\delta \bar{\omega}$
I. $\diamond \delta \bar{\omega} \mu \varepsilon \nu$
2. $\delta \bar{\varphi} \varsigma, \delta \circ \bar{\zeta} \varsigma$
2. $\delta \bar{\omega} \tau \varepsilon$
3. $\delta \bar{\varphi}, \delta \circ i, \delta \omega \omega n$
3. $\delta \bar{\omega} \sigma t$

Some forms like $\delta \omega \sigma n, \delta \omega \sigma \omega \mu \varepsilon \nu$ are probably aorist subjunctives from a first aorist $\varepsilon_{\delta \omega \sigma \alpha}$ (found in the papyri).

3. Imperative:

Singular
2. $\delta \delta s$
3. $\delta \delta ่ \omega$

Plural

2. ธórs

3. $[\delta o ́ \tau \omega \sigma \alpha \nu]$

4. Infinitive:

\section{Soüvat}

5. Participle:

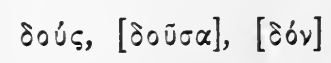

Declined like the present act. participle. 
475. The aorist middle of $\delta i \delta \omega \mu$ is:

I. Indicative:

Singular

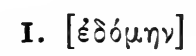

2. ["ूou

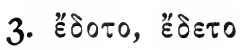

\section{Plural}

I. $[\varepsilon \delta \delta \rho \varepsilon \theta \alpha]$

2. " $\varepsilon 000 \varepsilon$

3. हैรับข

2. Subjunctive:

Does not occur in the New Testament.

3. Imperative:

Does not occur in the New Testament.

4. Infinitive:

Does not occur in the New Testament.

5. Participle:

Does not occur in the New Testament.

476. Some $\omega$-verbs have aorists conjugated like those of $\mu$-verbs.

I. The aorist ( $\mu_{l}$-aorist) indicative active of $\gamma(\nu \omega \dot{\sigma} \times \omega$ is:
Singular
Plural
I. "̈
I. "̋ $\gamma \nu \omega \mu \varepsilon \nu$
2. "̈ $\gamma$ $\%$ s
2. है $\gamma \nu \omega \tau \varepsilon$
3. "้ $\gamma \nu \omega$
3. "

2. The subjunctive is $\gamma \nu \tilde{\omega}, \gamma \gamma \tilde{\varphi} \varsigma$, etc., with $\omega$ throughout. But third sing. is rvoi.

3. The imperative is $\gamma \nu \bar{\omega} \theta \imath, \gamma \nu \dot{\omega} \tau \omega, \gamma \nu \bar{\omega} \tau \varepsilon,[\gamma \nu \omega \dot{\omega} \tau \omega \sigma \alpha \nu]$

4. The infinitive is $\gamma \nu \bar{\omega} v \alpha$

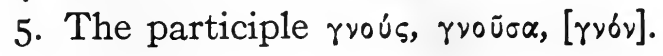




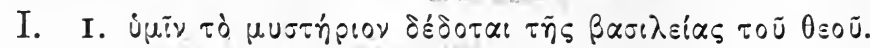

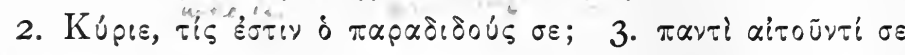

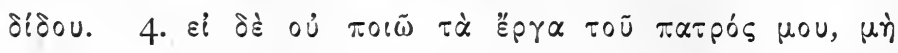

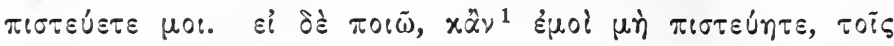

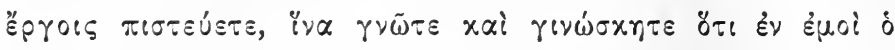

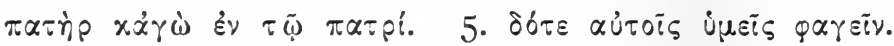

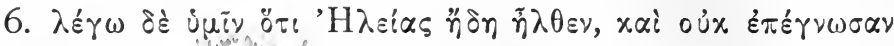

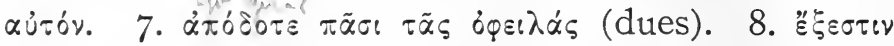

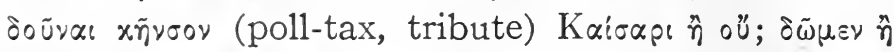
$\mu \grave{\eta} \delta \omega \mu \varepsilon \nu$;

II. I. If I know all mysteries and have not love, I am nothing. 2. I give you power to cast out demons. 3. Jesus was giving bread to the disciples that they might give it to the multitude.

\section{LESSON LVI}

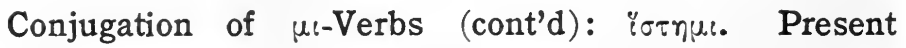
Imperative of eipi. Second Aorist of $\beta \alpha i v \omega$ $\varphi \eta \mu i$ 478. VOCABULARY

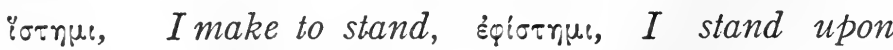
place, stand or by, come

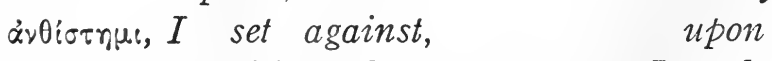
withstand $\quad x \alpha \theta i \sigma \tau r, \mu$, I set down, apḋvírnu, I raise up, rise, arise

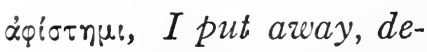
part from

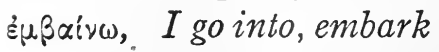
point $\mu \varepsilon \tau \alpha \beta x^{i}(v \omega, I$ pass over, depart

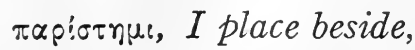
stand by

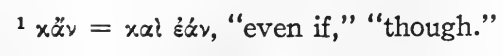




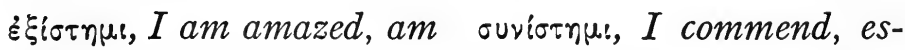
beside myself tablish

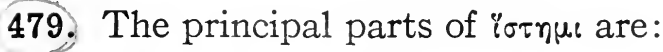

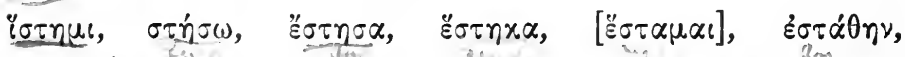
second aor. act. हैornv.

Observe that: I. The verb-stem is $\sigma \tau \alpha-2$. The present stem is the verb-stem reduplicated, with the

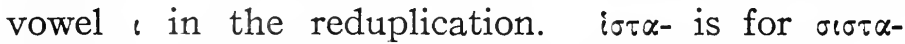
(initial $\sigma$ is represented by the rough breathing).

480. The conjugation of i $\sigma \tau \eta \mu \iota$ in the present active is:

I. Indicative:

Singular

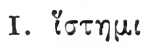

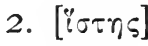

3. "iฮrvo

\section{Plural}

I. $[i \sigma \tau \alpha \mu \varepsilon \nu]$

2. $[i \sigma \tau \alpha \tau \varepsilon]$

3. $[i \sigma \tau \tilde{\alpha} \sigma l]$

Many forms from i $\sigma \tau \dot{\alpha} \nu \omega$ occur. They are regular in their conjugation.

2. Subjunctive:

Does not occur in the New Testament.

3. Imperative:

Does not occur in the New Testament.

4. Infinitive:

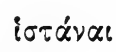

5. Participle:

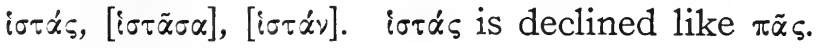

481. Imperfect indicative active forms of i $\sigma \tau \eta \mu \iota$ do not occur in the New Testament. 
482. The conjugation of the present middle and passive of " $\sigma \tau \eta \mu$ เ is:

I. Indicative:

\section{Singular}

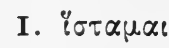

2. "ช $\sigma \tau \sigma \alpha b$

3. "ซ $\tau \alpha \tau \alpha$ t

\section{Plural}

I. $i \sigma \tau \dot{\alpha} \mu \varepsilon \theta \alpha$

2. $\check{\sigma \sigma \tau \alpha \sigma \theta \varepsilon}$

3. "ซ $\tau \alpha \nu \tau \alpha$.

2. Subjunctive:

Does not occur in the New Testament.

3. Imperative:

Singular

2. " $\sigma \tau \alpha \sigma 0$

3. $[i \sigma \tau \alpha \dot{\alpha} \sigma \omega]$

4. Infinitive:

5. Participle:

\section{Plural}

2. $[\because \sigma \tau \alpha \sigma \theta \varepsilon]$

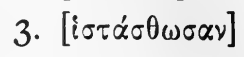

\section{$\imath^{\prime} \sigma \tau \sigma \theta \alpha \iota$}

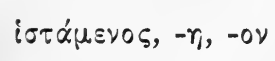

483. The imperfect indicative middle and passive of iotrue is:

\section{Singular}

I. i $\sigma \tau \alpha \dot{\alpha} \mu \eta \nu 1$

2. $[i \sigma \tau \alpha \sigma 0]$

3. " $\sigma \tau \alpha \tau 0$

\section{Plural}

I. $i \sigma \tau \dot{\alpha} \mu \varepsilon \theta \alpha$

2. "ซ $\sigma \alpha \sigma \theta \varepsilon$

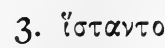

484. $q \eta \mu$, $I$ say, is found in the pres. ind. act. first pers. sing. $\varphi \eta \mu i$, third pers. sing. $\varphi$ noi, third pers. plur. $q$ ooi, and in the imperfect ind. act. third pers.

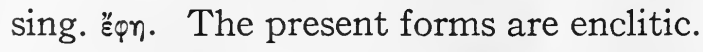

485. The present imperative of si $\mu$, $I \mathrm{am}$, is:

Singular

2. $i \sigma \theta$ l

3. है $\sigma \tau \omega, \not ु น \omega$

\section{Plural}

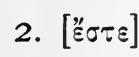

3. है $\sigma \omega \sigma \alpha \nu$

1 Long i. See 70, 2. 
486. The aorist ( $\mu l$-aorist) active of $i \sigma \tau \eta \mu l$ is:

I. Indicative:
Singular
Plural

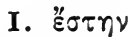
I. $\varepsilon \sigma \tau \eta \mu \varepsilon \nu$
2. ["ॅัซทs]

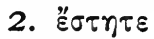
3. हैं๘ฑ
3. है $\sigma \tau \eta \sigma \alpha \nu$

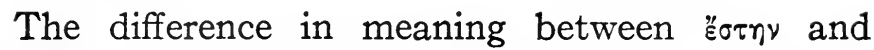

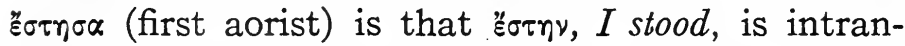

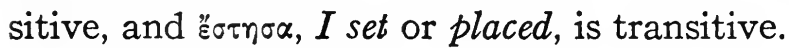

2. Subjunctive:
Singular
Plural
I. $[\sigma \tau \tilde{\omega}]$
I. $[\sigma \tau \tilde{\omega} \mu \varepsilon v]$
2. $[\sigma \tau \tilde{n} s]$
2. $\sigma \tau \tilde{\eta} \tau \varepsilon$
3. $\sigma \tau \bar{n}$
3. $\sigma \tau \tilde{\omega} \sigma \ell$

3. Imperative:
Singular
Plural
2. $\sigma \tau \tilde{\eta} \theta_{l}$,
2. $\sigma \tau \tilde{\eta} \tau \varepsilon$
3. $\sigma \tau \dot{\eta} \tau \omega$
3. $[\sigma \tau \dot{\eta} \tau \omega \sigma \alpha \nu]$

$-\sigma \tau \alpha$ occurs only in compounds.

4. Infinitive:

$$
\sigma \tau \tilde{\eta} \nu \alpha \iota
$$

5. Participle:

$$
\sigma \tau \alpha^{\prime} \zeta, \quad[\sigma \tau \tilde{\alpha} \sigma \alpha], \quad\left[\sigma \tau \alpha^{\prime} \nu\right]
$$

$\sigma \tau \dot{\alpha} \varsigma$ is declined like $\pi \tilde{\alpha} \varsigma$.

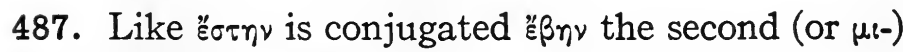
aorist of $\beta \alpha i v \omega$. Thus:

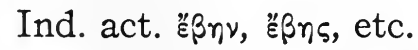

Subj. act. third sing. $\beta \tilde{n}$.

Imperative act. $\beta \bar{\eta} \theta_{l}$ and $-\beta \alpha, \beta \alpha^{\prime} \tau \omega,-\beta \alpha \tau \varepsilon$. 
Infinitive act. $\beta \tilde{\eta}$ val

Participle act. $\beta \alpha ́ s$, declined like $\pi \tilde{\alpha} \varsigma$.

In the New Testament $\beta \alpha^{i}$ ( $\omega$ occurs only in compounds (see vocabulary).

488.

EXERCISES

I. I. $x \alpha \grave{l} \dot{\alpha} \nu \alpha \sigma \tau \dot{\alpha} \varsigma \tilde{\eta} \lambda \theta \varepsilon \nu \pi \rho \dot{s} \varsigma \tau \dot{\nu} \nu \pi \alpha \tau \dot{\varepsilon} \rho \alpha \dot{\varepsilon} \alpha u \tau 0 \tilde{u}$. 2. $x \alpha \dot{l}$

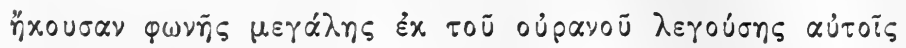

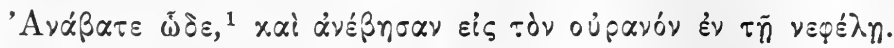

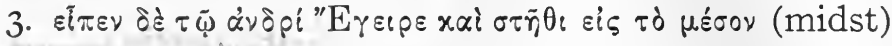

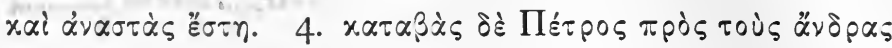

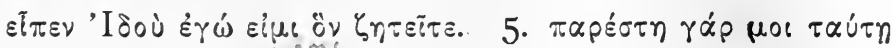

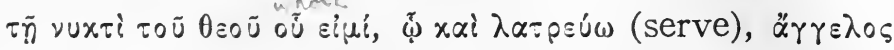

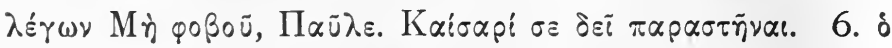

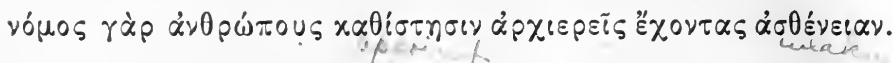

II. I. An angel stood by Paul in the night. 2. Two men went up into the temple. 3. Who appointed you a ruler of the people? 4. The disciples went into the boat.

\section{LESSON LVII}

Conjugation of $\mu t-$ Verbs (Continued): $\tau i \theta \eta \mu \iota$

489.

VOCABULARY

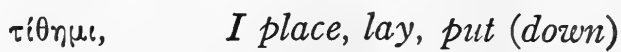

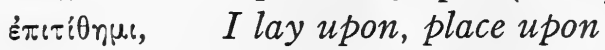

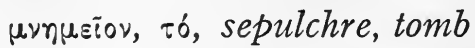

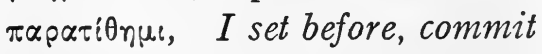

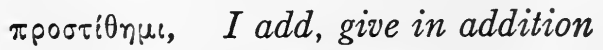

$1 \omega \delta \varepsilon$, adv. hither, here. 
490. The principal parts of $\tau i \theta \eta \mu \iota$ are:

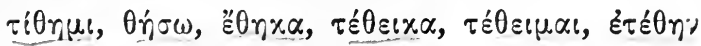

Observe that: I. The verb-stem is $\theta \varepsilon-$. 2. The present stem is the reduplicated verb-stem, with the vowel, in the reduplication. 3. The aorist indic. has $-x \alpha$ as suffix.

491. The present active of $\tau i \theta n \mu \iota$ is:

I. Indicative:
Singular
Plural
I. $\tau i \theta \eta \mu \iota$
I. $\tau\{\theta \varepsilon \mu \varepsilon \nu$
2. $[\tau i \theta n s]$
2. $\tau i \theta \varepsilon \tau \varepsilon$

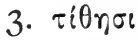
3. $\tau \imath \theta \varepsilon \alpha \sigma t$

2. Subjunctive:
Singular
Plural
I. $\tau \iota \theta \dot{\omega}$
I. $\tau t \theta \tilde{\omega} \mu \varepsilon \nu$
2. $\tau i \theta \tilde{n} s$
2. $\tau: \theta \tilde{\eta} \tau \varepsilon$
3. $\tau i \theta \tilde{n}$
3. $\tau \imath \theta \tilde{\omega} \sigma \iota$

3. Imperative:
Singular
Plural
2. $\tau i \theta \varepsilon \imath$
2. $\tau i \theta \varepsilon \tau \varepsilon$
3. $\tau \iota \theta \varepsilon \dot{\varepsilon} \tau \omega$

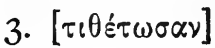

4. Infinitive:

$$
\tau \iota \theta \varepsilon \dot{\varepsilon} \propto \iota
$$

5. Participle:

$$
\tau \imath \theta \varepsilon i \varsigma, \tau i \theta \varepsilon \tau \sigma \alpha, \tau \imath \theta \varepsilon \nu
$$

Declined like the aorist passive participle of $\lambda u ́ \omega: \lambda u \theta \varepsilon i \varsigma,-\varepsilon i \sigma \alpha,-\varepsilon \dot{v},(350)$. 
492. The imperfect indicative active of $\tau(\theta \eta n \mu$ is:

Singular

I. [ย̇̃

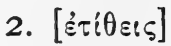

3. Ėं
Plural

I. $[\varepsilon \tau i \theta \varepsilon \mu \varepsilon \nu]$

2. $[\dot{\varepsilon} \tau i \theta \varepsilon \tau \varepsilon]$

3. ह่ $\tau i \theta \varepsilon \sigma \alpha \nu$

A third pers. plur. żं $\theta_{0} 0 u \nu$ is from $\tau \imath \theta \varepsilon \dot{\varepsilon} \omega$.

493. The conjugation of the present middle and passive of $\tau i \theta \eta_{\mu} \mu r$ is:

I. Indicative:

Singular
I. $\tau i \theta \varepsilon \mu \alpha \imath$
2. $[\tau i \theta \varepsilon \sigma \alpha i]$
3. $\tau i \theta \varepsilon \tau \alpha \ell$
I. $[\tau \imath \theta \varepsilon \mu \varepsilon \theta \alpha]$
2. $\tau i \theta \varepsilon \sigma \theta \varepsilon$
3. $\tau i \theta \varepsilon \nu \tau \alpha \iota$

Plural

2. Subjunctive:

Does not occur in the New Testament.

3. Imperative:

Singular
Plural
2. $[\tau i \theta \varepsilon \sigma \theta \varepsilon]$
3. $\tau: \theta \varepsilon \dot{\varepsilon} \sigma \omega \sigma \alpha \nu$

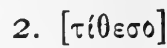

3. $[\tau \iota \theta \varepsilon \dot{\varepsilon} \sigma \omega]$

4. Infinitive:

$$
\tau\{\theta \varepsilon \sigma \theta \alpha \iota
$$

5. Participle:

$$
\tau \ell \theta \varepsilon \mu \varepsilon \nu \circ \varsigma,-\eta,-\circ \nu
$$

494. The imperfect indicative middle and passive of $\tau^{i} \theta \eta \mu \varepsilon$ is:

\section{Singular}

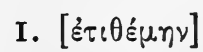

2. $[\dot{\varepsilon} \tau \hat{\imath} \theta \varepsilon \sigma 0]$

3. É $\tau i \theta \varepsilon \tau 0$

\section{Plural}

I. $[\varepsilon \dot{\tau} \imath \theta \varepsilon \dot{\varepsilon} \mu \varepsilon \alpha \alpha]$

2. $[\dot{\varepsilon} \tau i \theta \varepsilon \sigma \theta \varepsilon]$

3. Eं $i \theta \varepsilon \nu \tau 0$ 
495. The aorist active of $\tau(\theta \eta \eta \mu$ is:

I. Indicative:

Singular
I. $" \theta n \times \alpha)$

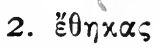
I. $\varepsilon \theta \dot{n} x \alpha \mu \varepsilon \nu$
3. "ै $\theta \eta x \varepsilon$
2. $\varepsilon \theta \dot{\eta} x \alpha \tau \varepsilon$
3. "ै $0 \eta x \alpha \nu$

Plural

As is the case with $\delta(\delta \omega \mu$, so $\tau i \theta \eta \mu$, has the $x$ aorist in the indicative active. Other modes are of the $\mu$ - aorist type.

2. Subjunctive:
Singular
Plural
I. $\theta \bar{\omega}$
I. $\theta \tilde{\omega} \mu \varepsilon \nu$
2. $\theta \tilde{n} s$
2. $[\theta \tilde{r} \tau \varepsilon]$
3. $\theta \tilde{n}$
3. $\theta \tilde{\omega} \sigma \iota$

3. Imperative:
Singular
Plural
2. $\theta \dot{\varepsilon} \varsigma$
2. $\theta \varepsilon ́ \tau \varepsilon$
3. $[\theta \dot{\varepsilon} \tau \omega]$
3. $[\theta \varepsilon \dot{\varepsilon} \tau \omega \sigma \alpha \nu]$

4. Infinitive:

\section{$\theta \varepsilon i \nu \alpha \iota$}

5. Participle:

Declined like $\tau \iota \theta \varepsilon i \varsigma$.

$$
\theta \varepsilon i \varsigma,[\theta \varepsilon i \sigma \alpha],[\theta \varepsilon \dot{\varepsilon} \nu]
$$

496. The aorist middle of $\tau\{\theta \eta \mu \iota$ is:

I. Indicative:
Singular
Plural
I. $\varepsilon$ éfunv)
I. $[\varepsilon \theta \varepsilon \dot{\varepsilon} \mu \varepsilon \alpha]$
2. है0०u
2. $" \varepsilon \theta \varepsilon \sigma \theta \varepsilon$
3. है $\theta \varepsilon \tau \circ$
3. . है $\theta \varepsilon \vee \tau 0$ 
2. Subjunctive:

Singular

I. $[\theta \tilde{\omega} \mu \alpha \iota]$

2. $[\theta \tilde{n}]$

3. $[\theta \tilde{r} \tau \alpha l]$

3. Imperative:

Singular

2. $\theta 0 \tilde{u}$

3. $[\theta \varepsilon \sigma \theta \omega]$
Plural

I. $\theta \dot{\omega} \mu \varepsilon \theta \alpha$

2. $[\theta \tilde{n} \sigma \theta \varepsilon]$

3. $[\theta \bar{\omega} \nu \tau \alpha l]$

2. $\theta \varepsilon \dot{\sigma} \sigma \theta \varepsilon$

3. $[\theta \varepsilon \varepsilon \sigma \theta \omega \sigma \alpha \nu]$

4. Infinitive:

$$
\theta \varepsilon ́ \sigma \theta \alpha \iota
$$

5. Participle

$$
\theta \varepsilon \dot{\mu} \mu \varepsilon \vee \circ \sigma^{-},-\eta,-O \nu
$$

497.

\section{EXERCISES}

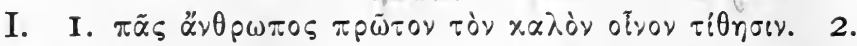

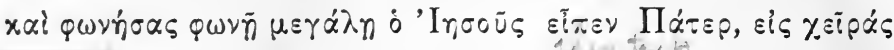

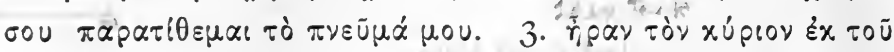

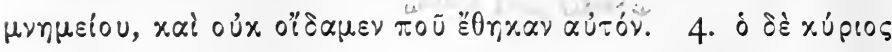

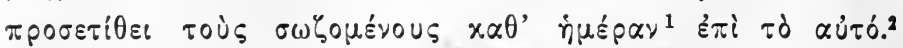

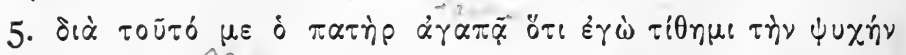

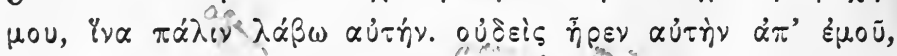

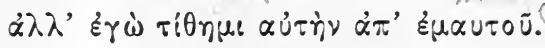

II. I. The good shepherd lays down his life for the sheep. 2. The apostle commends the disciple to the Lord. 3. I do not know where they laid him. 4. He was placing his hands upon the children.

Note: The students may now begin to read I John and continue it as Lessons after Lesson LIX is finished.

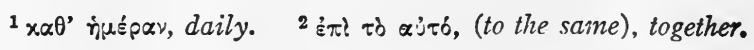




\section{LESSON LVIII}

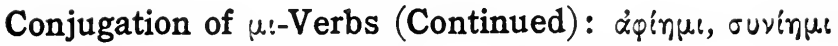

\section{Other Verbs: Old Forms}

498.

VOCABULARY

גoinu, I send away, for- ouvinu, I perceive, undergive, leave, let

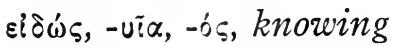
$\dot{\varepsilon} \sigma \tau \omega \dot{\omega} \varsigma, \tilde{\omega} \sigma \alpha, \dot{o} \varsigma$, standing

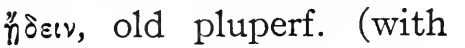
stand

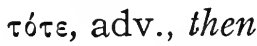
ir $r$ r ov, second aor. ind. act. of $\ddot{\alpha} \gamma \omega$

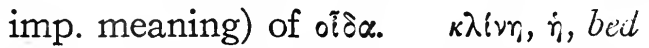

499. The verb $i r \mu$, I send, occurs in the New Testament only in compounds. Of this verb the most common compounds are $\dot{\alpha} \varphi i_{\eta} \mu_{\ell}\left(\dot{\alpha} \pi \dot{o}+i \eta \mu_{l}\right)$ and ouvinus.

The verb-stem of in $\mu$ is $\dot{\varepsilon}$-. The present stem is the reduplicated verb-stem, with $:$ in the reduplication.

The principal parts of $\alpha \varphi$ in $\mu$ are:

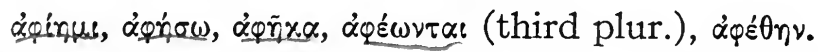
500. The following forms of $\alpha$ i in $\mu$ are those which occur most frequently in the New Testament.

Present indicative active:

Singular
I. [ápinul]
I. $\dot{\alpha} \varphi \hat{\imath} \varepsilon \mu \varepsilon \nu, \dot{\alpha} \varphi \hat{\imath} \propto \mu \varepsilon \nu$
2. $\dot{\alpha} \varphi \tilde{\varepsilon} \bar{s}$ (from $\dot{\alpha} \varphi \hat{i} \omega)$
3. גipinot
2. $\alpha \varphi^{\prime} \varepsilon \tau \varepsilon$
3. ¿̀piovor

Imperfect indicative active:

Third sing. $\ddot{\eta} \phi เ \varepsilon \nu$ (notice augment of the preposition) 
Present active imperative:

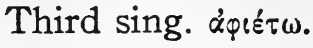

Present active infinitive:

$$
\text { ápréva! }
$$

Present indicative middle and passive:

Singular

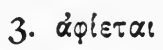

Plural

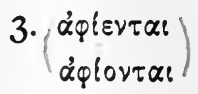

The aorist ( $x$-aorist) indicative active $\dot{\alpha} \varphi \tilde{\eta} x \alpha$ is conjugated like $\varepsilon^{\prime} \theta \eta \times \alpha$.

The second ( $\left.\mu_{-}\right)$aorist subjunctive active:
Singular
Plural
I. $\dot{\alpha} \varphi \tilde{\omega}$
I. -
2. -
2. $\dot{\alpha} p \tilde{\eta} \tau \varepsilon$
3. $\alpha \dot{\varphi} \tilde{n}$
3. $\alpha \dot{\phi \omega \sigma t}$

The second aor. imperative active:

Singular

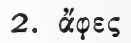

Plural

2. $\ddot{\alpha} \varphi \varepsilon \tau \varepsilon$

The second aor. infinitive active $\alpha \varphi \varepsilon i \nu \alpha$, The second aor. participle active dipzis (masc.).

501. The following forms of ouvinus occur.

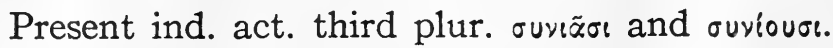

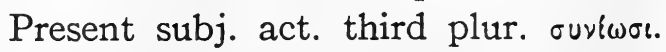

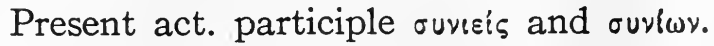

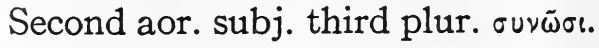

502. The verb ä $\gamma \omega$ has a reduplicated second aorist ทrarov.

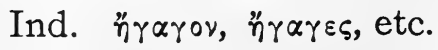

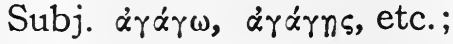


and the rest like $\varepsilon^{\prime}{ }^{\prime} \pi \circ \nu$, except the reduplication throughout the aorist.

503. The old pluperfect of oid $\alpha$ with the meaning of the imperfect is

I. Indicative active:

Singular

I. $\ddot{\delta} \varepsilon เ v, I$ knew

2. $\ddot{\delta} \delta \varepsilon ! 5$

3. $\ddot{\delta} \delta \varepsilon$
Plural

I. $[\ddot{n} \delta \varepsilon(\mu \varepsilon v]$

2. $\ddot{n} \delta \varepsilon เ \tau \varepsilon$

3. $\ddot{\delta} \delta \varepsilon เ \sigma \alpha \nu$

2. The old perfect subjunctive (with meaning of the present) of this verb is

ACTIVE

Singular
I. $\operatorname{\varepsilon i\delta \tilde {\omega }}$

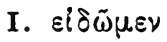
2. $\varepsilon i \delta \tilde{n} s$
3. $\varepsilon i \delta \tilde{n}$
2. $\varepsilon i \delta \tilde{\eta} \tau \varepsilon$
3. $[\varepsilon i \delta \tilde{\omega} \sigma t]$

\section{Plural}

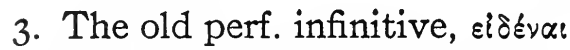

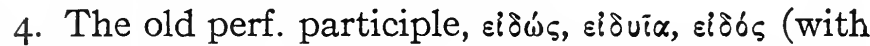
meaning of the present). Declined like $\lambda \varepsilon \lambda u \times \omega \dot{ }$, -vĩa, -ós.

504. An old perfect active participle from " $\sigma \tau \eta \mu:$ is found also in the New Testament.

Nom. $\dot{\varepsilon} \sigma \tau \omega \varsigma, \quad \dot{\varepsilon} \sigma \tau \tilde{\omega} \sigma \alpha, \quad \dot{\varepsilon} \sigma \tau \delta \varsigma$

Gen. $\quad \dot{\varepsilon} \sigma \tau \tilde{\omega} \tau \circ \varsigma$, $\dot{\varepsilon} \sigma \tau \dot{\omega} \sigma \eta \varsigma, \varepsilon \dot{\varepsilon} \sigma \tau \tilde{\omega} \tau \circ \varsigma$

The other cases can be easily formed from these. غ̇øน's has the intensive meaning, standing.

505.

EXERCISES

I. I. $\delta \mu \iota \sigma \theta \omega \tau \dot{\delta} s$ (hireling) $x \alpha i$ oủx $\bar{\omega} \nu \pi 0 เ \mu \dot{\eta} \nu$, ờ oủx

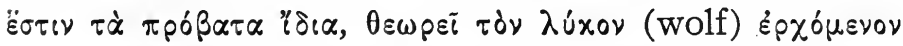

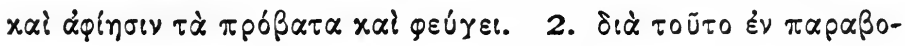




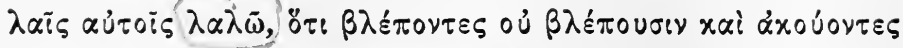

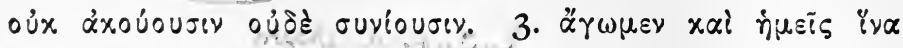

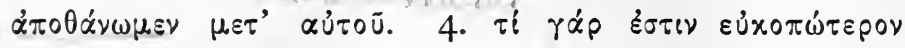

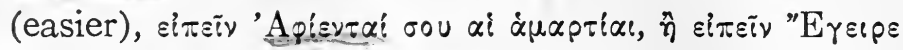

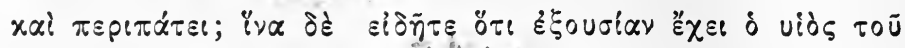

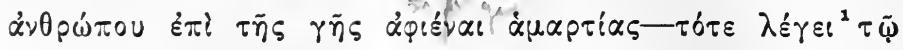

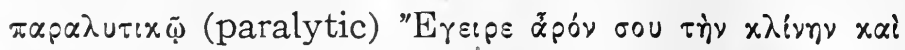

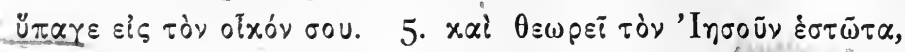

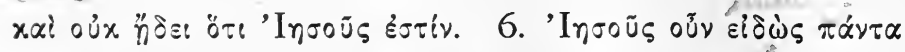

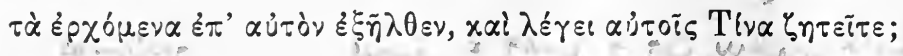

II. I. We saw him standing in the boat. 2. He said to the man, "Thy sins are forgiven." 3. I knew not who it was. 4. They led the servants to the house.

\section{LESSON LIX}

\section{The Optative Mode. Wishes}

506.

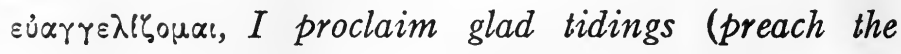
gospel)

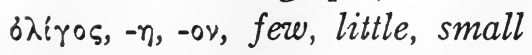

$\pi \alpha$ pousia, $\dot{\eta}$, coming, presence

on

onjuspov, adv., to-day, this day

507. Besides the indicative, subjunctive, and imperative modes, there is another mode in Greek, called the Optative.

In meaning the optative is a sort of weaker subjunctive. The subjunctive and optative are really different forms of the same mode. the mode of hesitating affirmation.

1 xגivn, $\dot{\eta}$, a couch, a bech 
508. In the New Testament the optative mode occurs only sixty-seven times, and in the present and aorist tenses only.

509. Of the forms of the optative mode found in the New Testament the following are representative:

I. Present tense (act. and middle):

Singular

Plural

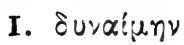

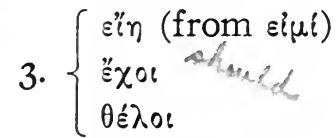
2. $\pi \dot{\alpha} \sigma \chi \circ เ \tau \varepsilon$

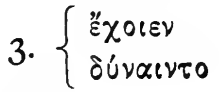

2. Second aorist (act. and middle):

Singular

Plural.

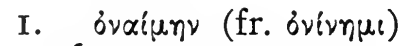

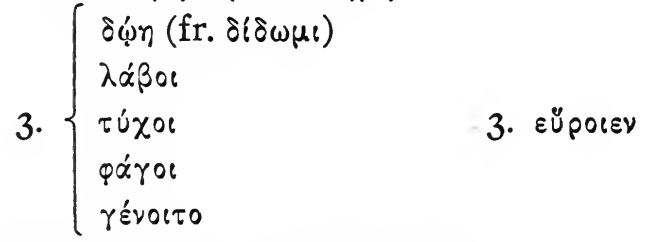

3. First aorist (act. and middle):

Singular

Plural

I. $\varepsilon \dot{u} \xi \alpha i \mu \eta \nu$

3. $\left\{\begin{array}{l}\pi \varepsilon \rho \iota \sigma \sigma \varepsilon u ́ \sigma \alpha \iota \\ \pi \lambda \varepsilon \sigma \nu \alpha^{\prime} \sigma \alpha \iota \\ x \alpha \tau \alpha \rho \tau i \sigma \alpha \iota \\ x \alpha \tau \varepsilon \cup \theta u ́ v \alpha \iota\end{array}\right.$

3. $\left\{\begin{array}{l}\pi 0 เ \dot{\eta} \sigma \alpha \iota \varepsilon \nu \\ \psi \eta \lambda \alpha \varphi^{\prime} \sigma \varepsilon \iota \alpha \nu\end{array}\right.$

4. First aorist passive:

Third sing., $\lambda_{0 \gamma} \sigma \theta \theta \sin , \pi \lambda \eta \theta u \nu \theta \sin , \tau \eta p \eta \theta \sin$.

Observe that: I. There are two mode signs for the optative, either ? or $n$. 2. \& is used with the-

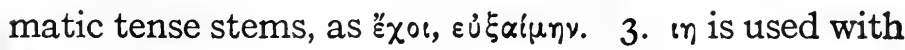


the non-thematic tense stems, as $\varepsilon i \eta, \delta \varphi \eta$. 4. $\quad \varepsilon$ and $\iota \alpha$ in the third person plural of both stems. 5. The mode sign ( $($ ) contracts with the vowel of the stem.

510. A wish about the future is usually expressed in the New Testament by the optative (generally the aorist), e.g.,

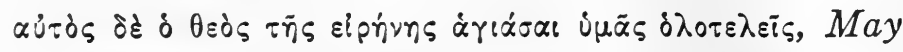
the God of peace himself sanctify you wholly.

The commonest wish of this kind is the phrase

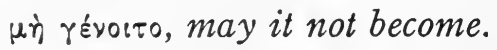

A wish about the future may be expressed by

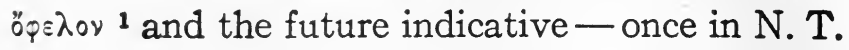

511. A wish about the present is expressed by ö $\varnothing \lambda_{\lambda \circ \nu}$ and the imperfect indicative, e.g.,

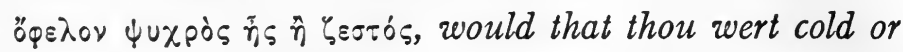
hot.

512. A wish about the past is expressed by ő $\varphi \varepsilon \lambda \circ v$ and the aorist indicative, e.g.,

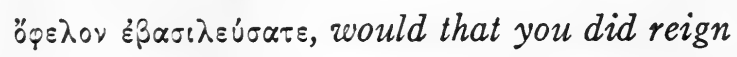

513. The fourth class condition is the condition undetermined and with remote prospect of determination. $\varepsilon i$ and the optative in the protasis, and the optative with $\ddot{\alpha} v$ in the apodosis. In the New Testament no whole example of this class of conditions occurs. There is found the condition (protasis) or the conclusion (apodosis), but not both at the same time.

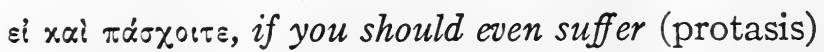

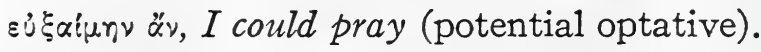

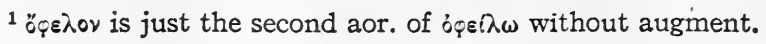




\section{PART II: ${ }^{1}$ SUPPLEMENT TO PART I}

${ }^{1}$ Part II is based on "A Grammar of the Greek New Testament in the Light of Historical Research," by A. T. Robertson. 


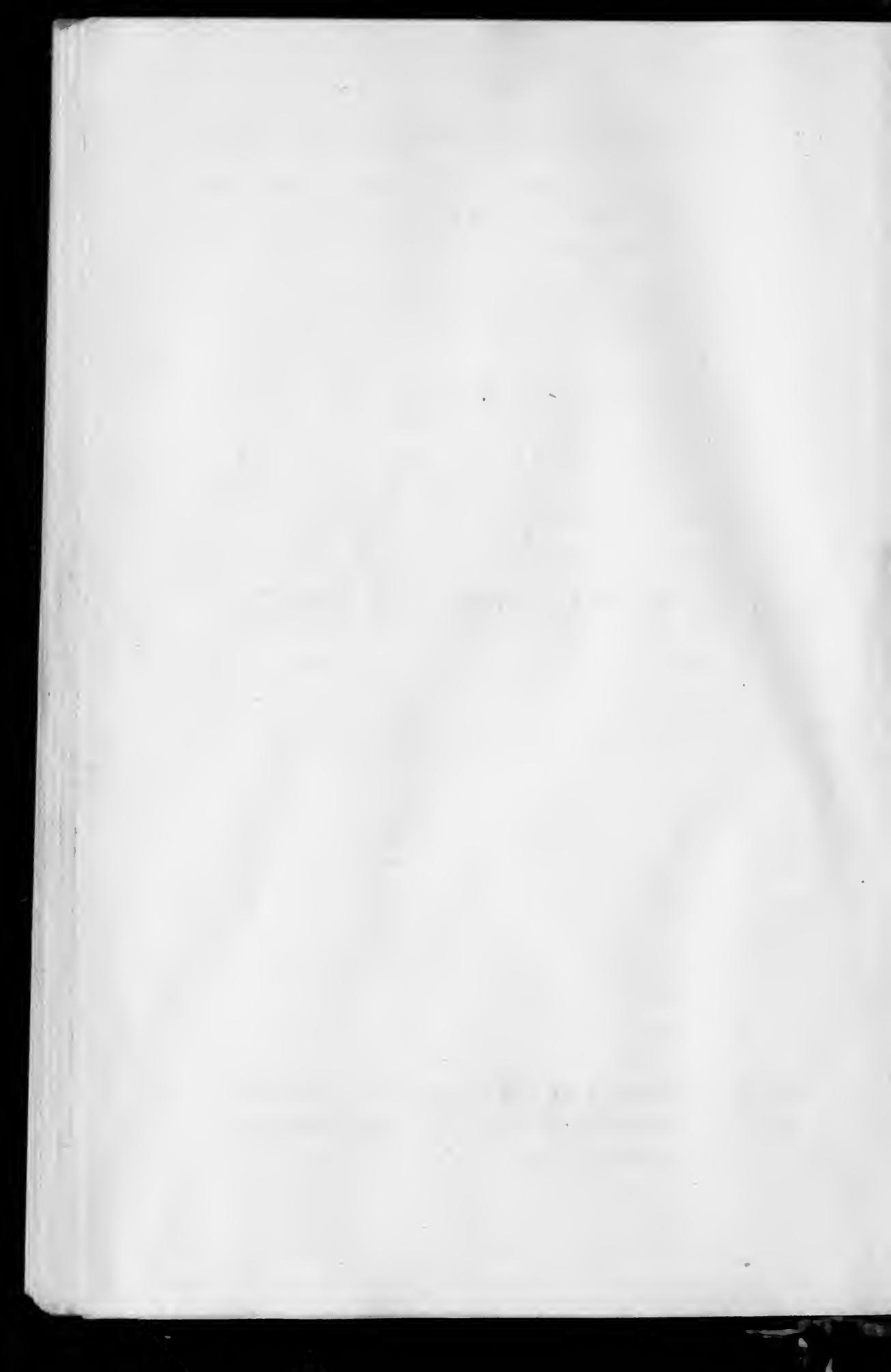




\section{A. Sounds and Writing}

$\S 1$. Syncope is the suppression of a short vowel between consonants for the sake of facility in pronunciation. Thus $\pi \alpha \tau$ pós for $\pi \alpha \tau$ épos.

$\S 2$. Diaeresis (separation) is indicated by a double dot ("), written over ? or $u$ to show that e or $u$ does not form a diphthong with the preceding vowel.

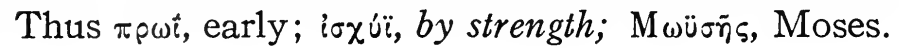

§3. Elision is the dropping of a short vowel at the end of a word before a word beginning with a vowel.

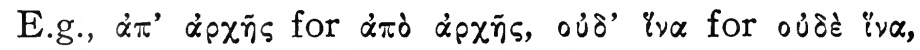
$\dot{\alpha} \varphi \dot{x} \dot{\varepsilon} \alpha u \tau \circ \tilde{u}$ for $\dot{\alpha} \pi \delta \dot{\varepsilon} \alpha u \tau \circ \tilde{u}$.

Note that an apostrophe marks the omission of the vowel.

$\S 4$. Crasis is the contraction of a vowel or diphthong at the end of a word with one at the beginning of the next word. Thus $x \dot{\alpha} \mu o i$ for $x \alpha i$ É $\mu \circ i ; x \dot{\alpha} x \in i v o s$ for

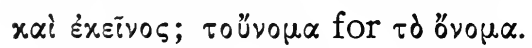

Note that crasis is indicated by the coronis (') over the contracted form.

$\S 5$. When a smooth mute $(\pi, x, \tau)$ is brought before the rough breathing by elision or in forming compounds, it is changed to the corresponding rough mute. This is called aspiration. Thus $\alpha^{\prime} \nu \theta^{\prime} \omega^{r} \nu$ for

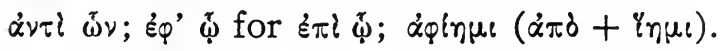


$\S 6$. The vowels $\alpha, \varepsilon, \circ$ are often interchanged in words of the same root. Sometimes there is an interchange among different vowels. This is called interchange or gradation of vowels. Thus $\pi \varepsilon i \theta \omega$,

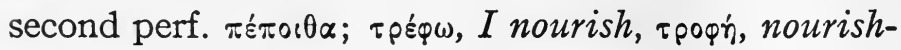

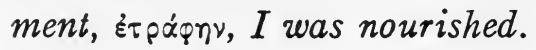

$\S 7$. Modern editors of Greek texts use four punctuation marks: the comma and period are used as in English; the interrogation mark (;) is in form like the English semicolon; the point above the line $\left(^{\cdot}\right)$ corresponds to the English semicolon or colon. 


\section{B. Paradigms of Nouns}

(a) Substantives

\$8. Some masculines in $-\alpha \varsigma$ of the first declension. ßoppãs, o, north (wind)

\section{Singular}

Nom. Boppãs So declined are some proper names in $-\alpha \varsigma$.
Gen. $\}$ Abl. $\}$ ßop $\tilde{\alpha}$
Loc.
Ins. $\}$ ßoppq̃
Dat.
Acc. Boppãv
Voc. $\beta$ ßop ã

§ 9. The normal form of contract substantives (those with stems in - $\varepsilon$ - or - 0 - of the second declension) may be seen in the masculine and neuter of the contract adjective $\delta\left\llcorner\pi \lambda_{0} \sigma \sigma_{\varsigma}\right.$ (\$I4).

Frequently these substantives are found in the un-

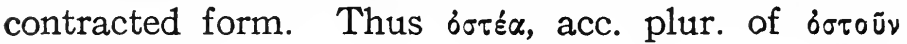

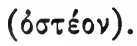

\$10. Substantives of the third declension with stems in -u-.

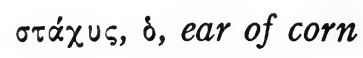




\section{Singular}

Nom. $\sigma \tau \alpha \dot{\chi} \cup \varsigma$

Gen. $\}$ Abl. $\sigma \tau \dot{\alpha} \chi \cup \circ \varsigma$

Loc.

Ins. $\} \sigma \tau \dot{\alpha} \chi u \ddot{\imath}$

Dat.

Acc. $\sigma \tau \alpha \dot{\chi} \chi u \nu$
Plural

$\sigma \tau \alpha \dot{\chi} \chi \cup \varepsilon \varsigma$

$\sigma \tau \alpha \chi \dot{u} \omega \nu$

$\sigma \tau \dot{\alpha} \chi \cup \sigma \iota$

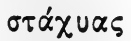

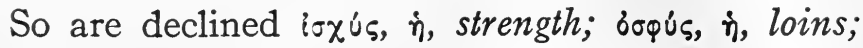

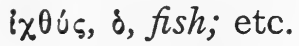

§11. Substantives of the third declension with stems in -ou- (-of-).

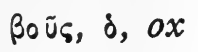

Singular

Nom. Boüs

Gen. $\}$ Bbl. $\}$ obs

Loc.

Ins.

Dat.

Acc. Boũy
Plural

$[\beta 6 \varepsilon \varsigma]$

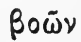

$[\beta \circ \cup \sigma t]$

Bóas

So are declined voũs, $\delta$, mind; $\pi \lambda \circ \tilde{\varsigma} \varsigma, \delta$, voyage; and $\chi \circ \tilde{u} \xi, \delta, d u s t$.

$\S 12$. The following substantives show some peculiarities, either of form or accent: to róvu, knee;

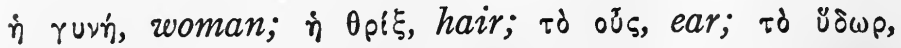
water, and $\delta$ xúwy, [xuvós], [xuví], [xúva]. Plu. xúves,

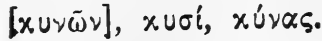




\section{PARADIGMS OF NOUNS}

Singular

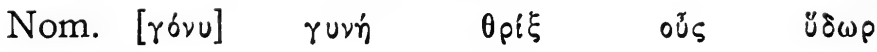

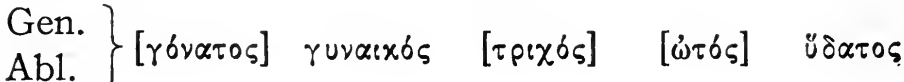
Loc.

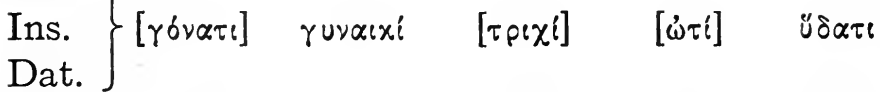

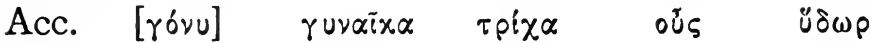

Voc. $\quad$ ívat

\section{Plural}

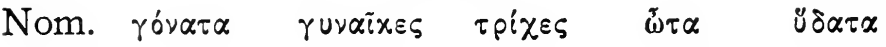

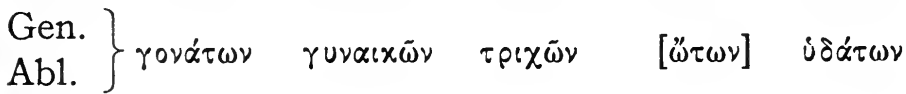

Loc.

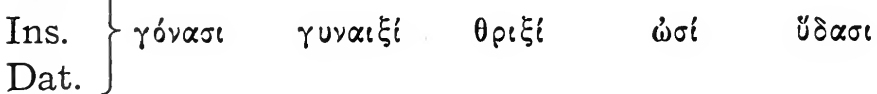

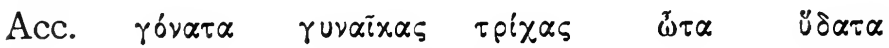

(b) Adjectives

$\S 13$. ¿o10s, one's own, and uixpós, small, of the $\alpha$ - and o-declension.

Singular

M. F. $\quad$ N. $\quad M . \quad F . \quad N$.

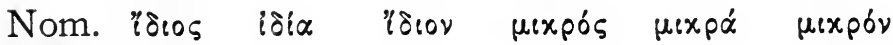

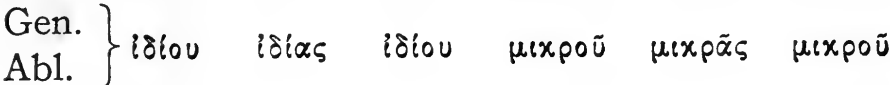
Loc.

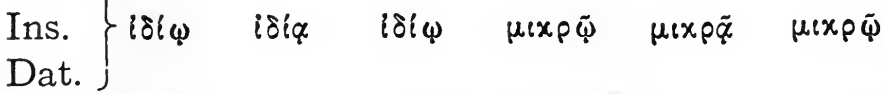

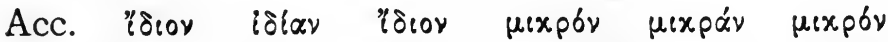

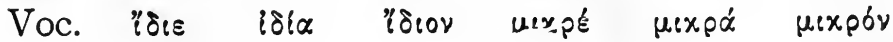




\section{Plural}

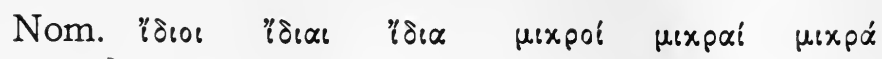

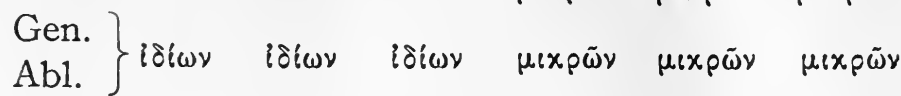
Loc.

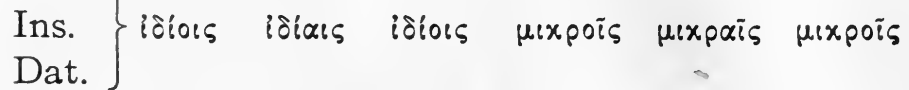

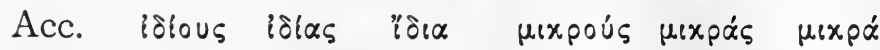
Voc. like nominative

$\S 14$. Contract adjectives of the $\alpha$ - and 0 - declension. $\delta เ \pi \lambda \circ \tilde{s} \varsigma$, twofold, double.

\begin{tabular}{|c|c|c|c|}
\hline \multicolumn{4}{|c|}{ Singular } \\
\hline & Masc. & Fem. & Neut. \\
\hline & $\delta\left(\pi \lambda_{00-}\right.$ & $\delta(\pi \lambda \circ \eta-$ & $\delta ! \pi \lambda \circ 00-$ \\
\hline Nom. & $\delta\left(\pi \lambda_{0} \tilde{u} \zeta\right.$ & $\delta เ \pi \lambda \tilde{\eta}$ & $\delta เ \pi \lambda \circ \tilde{u} y$ \\
\hline $\left.\begin{array}{l}\text { Gen. } \\
\text { Abl. }\end{array}\right\}$ & $\delta เ \pi \lambda \circ \tilde{U}$ & $\delta i \pi \lambda \tilde{\eta} s$ & $\delta ! \pi \lambda \circ \tilde{u}$ \\
\hline Loc. & & & \\
\hline $\begin{array}{l}\text { Ins. } \\
\text { Dat. }\end{array}$ & $\delta \iota \pi \lambda \tilde{\varphi}$ & $\delta(\pi \lambda \tilde{n}$ & $\delta \ell \pi \lambda \tilde{\varphi}$ \\
\hline Acc. & $\delta เ \pi \lambda \circ \tilde{u} \nu$ & $\delta(\pi \lambda \tilde{\eta} \nu$ & $\delta\left(\pi \lambda_{0} \tilde{u} \nu\right.$ \\
\hline \multicolumn{4}{|c|}{ Plural } \\
\hline Nom. & $\delta ! \pi \lambda_{0} i$ & $\delta เ \pi \lambda \alpha \tilde{\imath}$ & $\delta เ \pi \lambda \tilde{\alpha}$ \\
\hline $\left.\begin{array}{l}\text { Gen. } \\
\text { Abl. }\end{array}\right\}$ & $\delta เ \pi \lambda \tilde{\omega} \nu$ & $\delta เ \pi \lambda \tilde{\omega} \nu$ & $\delta เ \pi \lambda \tilde{\omega} \nu$ \\
\hline $\begin{array}{l}\text { Loc. } \\
\text { Ins. } \\
\text { Dat. }\end{array}$ & $\delta เ \pi \lambda_{0} i s$ & $\delta เ \pi \lambda \alpha i s$ & $\delta: \pi \lambda \circ i \varsigma$ \\
\hline Acc. & $\delta เ \pi \lambda \circ \tilde{U} \zeta$ & $\delta เ \pi \lambda \tilde{\alpha} \varsigma$ & $\delta เ \pi \lambda \tilde{\boldsymbol{\alpha}}$ \\
\hline
\end{tabular}

Of like form are those whose stems end in $\varepsilon$. 


\section{PARADIGMS OF NOUNS}

$a$. If $\varepsilon$,, , or $\rho$ precedes the stem vowel, $\alpha$ is found in the fem. sing. instead of $\eta$ (sometimes $\eta$ occurs).

So are declined-

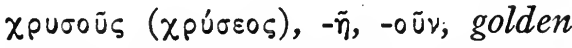

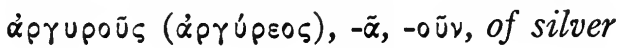

$\S 15$. Adjectives (of the third declension) with stem in -u-are declined like $\zeta \xi u ́ \varsigma$, sharp.

$$
\text { Singular Plural }
$$

M. F. N. M. F. $N$.

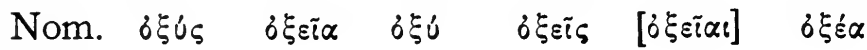

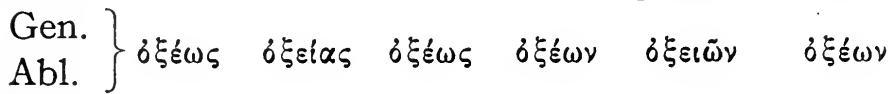
Loc.

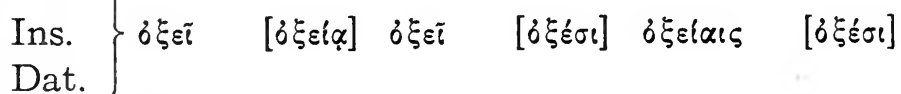

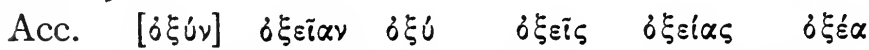

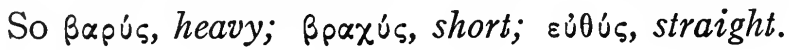

$\S 16$. Most of the participles with stems in -ovrare declined like the present participle of siui. Thus:

$$
M . \quad F . \quad N . \quad M . \quad F . \quad N \text {. }
$$

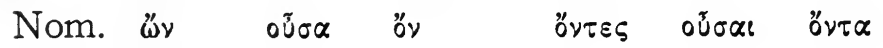

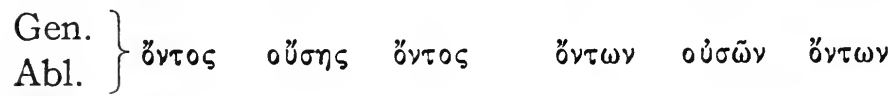

Loc.

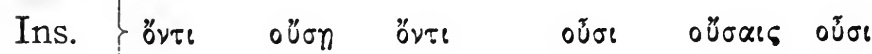

Dat.

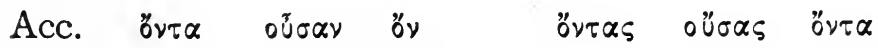




\section{Pronouns}

$\S 17$. A few forms of the demonstrative pronoun ¿ $\delta \varepsilon, \eta \eta \delta \varepsilon, \tau \delta \delta \varepsilon$, this, occur in the New Testament. It is declined like the article ( $\delta)$ with the enclitic $\delta \varepsilon$ added.

$\S 18$. Most other pronouns (not personal) are declined in the first and second declensions, and may be easily learned, as they are needed, from a lexicon. 
D. Paradigms of the Verb

§ 19. Simple $\omega$-verb. $\lambda u ́ \omega$.

\section{Active VoIce}

\begin{tabular}{|c|c|c|c|}
\hline \multirow{3}{*}{$\begin{array}{l}\text { INDIC. } \\
\mathrm{S} .\end{array}$} & Present & Imperfect & Future \\
\hline & I. $\lambda \dot{v} \omega$ & 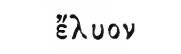 & $\lambda \dot{\sigma} \sigma \omega$ \\
\hline & 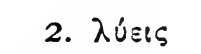 & है入 & $\lambda$ \\
\hline & 3. $\lambda \dot{\varepsilon \varepsilon}$ & 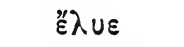 & $\lambda u ́ \sigma \varepsilon !$ \\
\hline & I. $\lambda u ́ o \mu \varepsilon \nu$ & $\varepsilon \lambda \cup ́ o \mu \varepsilon \nu$ & $\lambda$ $\lambda \dot{\sigma o \mu \varepsilon \nu}$ \\
\hline P. & 2. $\lambda u ́ \varepsilon \tau \varepsilon$ & $\varepsilon$ & 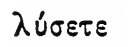 \\
\hline & 3. Xúovor & है入 U०ข & 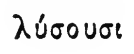 \\
\hline SuBJ. & I. $\lambda \dot{v} \omega$ & & \\
\hline S. & 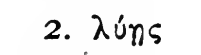 & & \\
\hline & 3. $\lambda u ́ n$ & & \\
\hline & I. $\lambda \dot{\omega} \omega \mu \varepsilon v$ & & \\
\hline $\mathrm{P}$ & 2. $\lambda u ́ \eta \tau \varepsilon$ & & \\
\hline & 3. $\lambda \dot{u} \omega \sigma \iota$ & & \\
\hline
\end{tabular}

Opt.

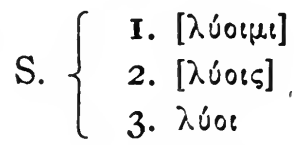

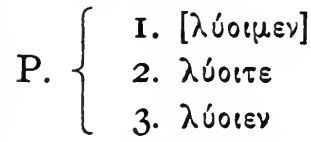

IMP.
S. \{
2. $\lambda \tilde{u} \varepsilon$

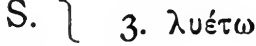
P. \{
2. $\lambda \cup ́ \varepsilon \tau \varepsilon$
3. $\lambda \cup \varepsilon ́ \tau \omega \sigma \alpha \nu$

INF.

PART

$$
\lambda \cup \dot{\varepsilon \varepsilon \nu}
$$


I Aorist I Perfect I Pluperf.

\begin{tabular}{|c|c|c|c|}
\hline INDIC. & I. हैं $\lambda \cup \sigma \alpha$ & 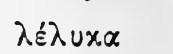 & {$[\varepsilon \lambda \lambda \varepsilon \lambda u ́ x \varepsilon เ \nu]$} \\
\hline S. & 2. है $\lambda \cup \sigma \alpha \varsigma$ & $\lambda \hat{\varepsilon} \lambda \cup x \alpha \varsigma$ & 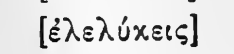 \\
\hline & 3. है $\lambda \cup \sigma \varepsilon$ & $\lambda \varepsilon \varepsilon^{\prime} \lambda u x \varepsilon$ & 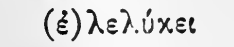 \\
\hline & I. $\varepsilon \lambda \lambda \dot{u} \sigma \alpha i \varepsilon \varepsilon \nu$ & $\lambda \varepsilon \lambda \dot{u} x \propto \alpha \varepsilon \varepsilon$ & 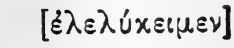 \\
\hline P. & 2. $\varepsilon_{\lambda} u ́ \sigma \alpha \tau \varepsilon$ & $\lambda \varepsilon \lambda \dot{u} x \alpha \tau \varepsilon$ & 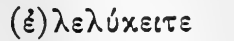 \\
\hline & 3. है & $\begin{array}{c}\lambda s \lambda u ́ x \alpha \sigma l, \\
\text { or }-\alpha \nu\end{array}$ & 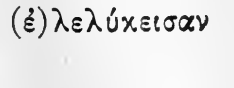 \\
\hline
\end{tabular}

SuBJ.

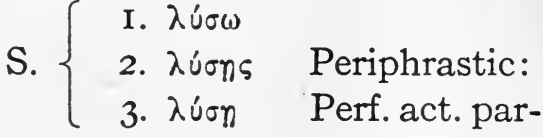
P. $\begin{cases}\text { I. } \lambda u ́ \sigma \omega \mu \varepsilon \nu & \text { ticiple and } \\ \text { 2. } \lambda u ́ \sigma \eta \tau \varepsilon & \text { pres. subj. of } \\ \text { 3. } \lambda u \sigma \omega \sigma, & \text { eipl. }\end{cases}$

Opt.
S. $\left\{\begin{array}{l}\text { I. }[\lambda \dot{v} \sigma \alpha ! u l] \\ \text { 2. }[\lambda \dot{v} \sigma \alpha t \xi] \\ \text { 3. } \lambda \dot{v} \sigma \alpha \ell\end{array}\right.$
P. $\left\{\begin{array}{l}\text { I. }[\lambda \dot{v} \sigma \alpha \iota \mu \varepsilon \nu] \\ \text { 2. }[\lambda \dot{v} \sigma \alpha \iota \tau \varepsilon] \\ \text { 3. } \lambda \dot{v} \sigma \varepsilon l \alpha \nu \text { or }-\alpha ! \varepsilon \nu\end{array}\right.$

IMP.
S. $\left\{\begin{array}{l}\text { 2. } \lambda \tilde{\sigma \sigma \sigma \nu} \\ \text { 3. } \lambda u \sigma \alpha \dot{\tau} \tau \omega\end{array}\right.$
P. $\left\{\begin{array}{l}\text { 2. } \lambda u \sigma \alpha \tau \varepsilon \\ \text { 3. } \lambda u \sigma \alpha ́ \tau \omega \sigma \alpha \nu\end{array}\right.$

\begin{tabular}{|c|c|c|}
\hline INF. & $\lambda \tilde{u} \sigma \alpha \ell$ & $\lambda \varepsilon \lambda u x \varepsilon ́ v \alpha l$ \\
\hline PART. & $\lambda \dot{u} \sigma \alpha \varsigma$ & $\lambda \varepsilon \lambda u x \omega ́ \varsigma,-v i \alpha,-o ́$ \\
\hline & $\lambda u ́ \sigma$ & \\
\hline & & \\
\hline
\end{tabular}


PARADIGMS OF VERBS

\section{Middle Voice}

\section{Present Imperfect Future}

INDIC.
S. \{
I. $\lambda \dot{u} \circ \mu \alpha_{i}$
$\varepsilon \lambda \cup \delta \mu \eta \eta$
$\lambda$ úoouas
2. $\lambda$ un
Ė่
$\lambda$ úon
3. $\lambda \dot{\varepsilon} \varepsilon \tau \alpha \varepsilon$

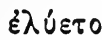
$\lambda \dot{U} \sigma \varepsilon \tau \alpha \ell$

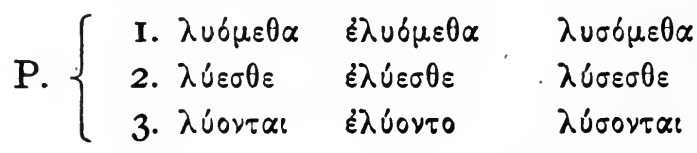

SuBJ.
S. $\left\{\begin{array}{l}\text { I. } \lambda \dot{u} \omega \mu \alpha \text { 2. } \lambda u ́ n \\ \text { 3. } \lambda \dot{n} \eta \tau \alpha \text { l }\end{array}\right.$
P. $\left\{\begin{array}{l}\text { I. } \lambda \cup \dot{\omega} \mu \varepsilon \theta \alpha \\ \text { 2. } \lambda \dot{\eta} \eta \sigma \theta \varepsilon \\ \text { 3. } \lambda \dot{v} \omega \nu \tau \alpha \iota\end{array}\right.$

OPT.

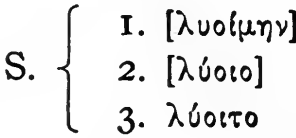

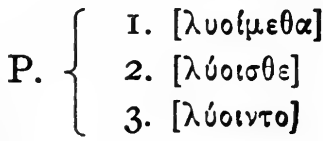

IMP.

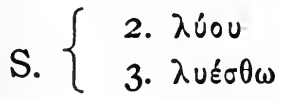

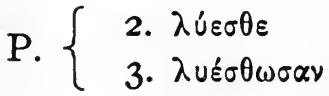

INF.

$\lambda \cup ́ \varepsilon \sigma \theta \alpha$

$\lambda \dot{\sigma} \sigma \varepsilon \sigma \theta \alpha \varepsilon$

PART.

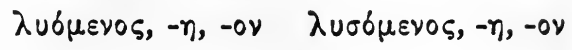




\section{BEGINNER'S GREEK GRAMMAR}

\section{Aorist I Perfect I Pluperf.}

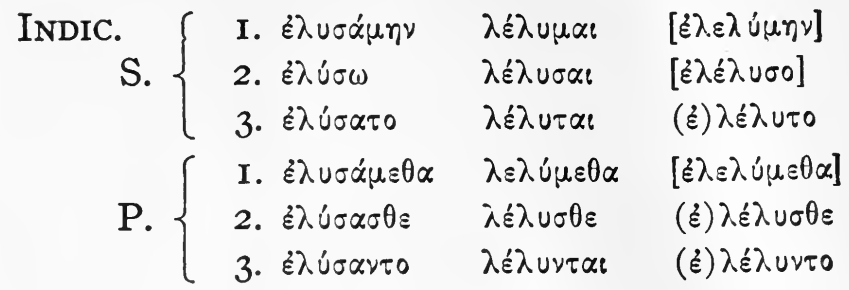

SubJ.
S. $\begin{cases}\text { I. } \lambda u ́ \sigma \omega \mu \alpha \ell & \\ \text { 2. } \lambda u ́ \sigma n & \text { Periphrastic: } \\ \text { 3. } \lambda u ́ \sigma \eta \tau \iota l & \text { Perf. midd. }\end{cases}$
P. $\begin{cases}\text { I. } \lambda \cup \sigma \dot{\omega} \mu \varepsilon \theta \alpha & \text { part. and } \\ \text { 2. } \lambda \dot{\sigma} \sigma \eta \sigma \theta \varepsilon & \text { subj. of sijul. } \\ \text { 3. } \lambda \dot{v} \sigma \omega \nu \tau \alpha \ell & \end{cases}$

Opt.
S. $\begin{cases}\text { I. } & \lambda \cup \sigma \alpha i \mu \eta \nu \\ 2 . & {[\lambda \dot{v} \sigma \alpha i 0]} \\ 3 . & {[\lambda \dot{v} \sigma \alpha i \tau 0]}\end{cases}$
P. $\left\{\begin{array}{l}\text { I. }[\lambda \cup \sigma \alpha i \mu \varepsilon \theta \alpha] \\ \text { 2. }[\lambda \dot{v} \sigma \alpha \cdot \sigma \theta \varepsilon] \\ \text { 3. }[\lambda \dot{v} \sigma \alpha เ v \tau \sigma]\end{array}\right.$

IMP.
S. \{
2. $\lambda \tilde{u} \sigma \alpha \ell$
$\lambda \varepsilon \dot{\varepsilon} \lambda \cup \sigma 0$
3. $\lambda \cup \sigma \alpha \dot{\sigma} \sigma \omega$
$[\lambda \varepsilon \lambda \dot{\sigma} \sigma \theta \omega]$
P. \{
2. $\lambda \cup \cup \sigma \sigma \theta \varepsilon$
3. $\lambda \cup \sigma \alpha \dot{\sigma} \theta \omega \sigma \alpha \nu$
$\lambda \varepsilon \dot{\varepsilon} \lambda \cup \sigma \theta \varepsilon$ $\lambda \dot{v} \sigma \alpha \sigma \theta \alpha \ell$
[ $[\lambda \varepsilon \lambda \dot{v} \sigma \theta \omega \sigma \alpha \nu]$

INF.

PART.

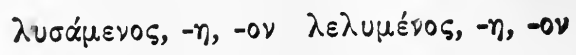

\section{PAssive Voice}

The passive voice of the present, imperfect, perfect and pluperfect tenses is the same in form as the middle. 
I Aorist

INDIC.

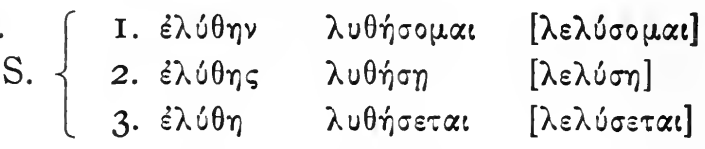

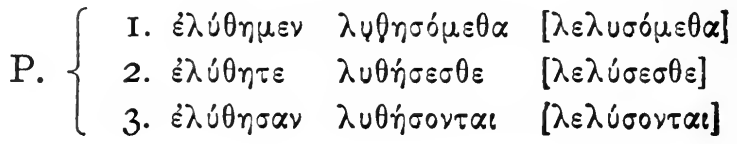

SubJ.
S. $\left\{\begin{array}{l}\text { I. } \lambda u \theta \tilde{\omega} \\ \text { 2. } \lambda u \theta \tilde{n} s \\ \text { 3. } \lambda u \theta \tilde{n}\end{array}\right.$
P. $\left\{\begin{array}{l}\text { I. } \lambda u \theta \tilde{\omega} \mu \varepsilon \nu \\ \text { 2. } \lambda u \theta \tilde{\eta} \tau \varepsilon \\ \text { 3. } \lambda u \theta \tilde{\omega} \sigma \iota\end{array}\right.$

OPT.
S. $\left\{\begin{array}{l}\text { I. }[\lambda u \theta \sin , \nu] \\ \text { 2. }[\lambda u \theta \operatorname{sir}, s] \\ \text { 3. } \lambda u \theta \sin \end{array}\right.$
P. $\begin{cases}\text { I. } & {[\lambda u \theta \sin \mu \varepsilon \nu]} \\ \text { 2. } & {[\lambda u \theta \sin \tau \varepsilon]} \\ \text { 3. } & {[\lambda u \theta \sin \sigma \alpha \nu]}\end{cases}$

IMP.
S. $\left\{\begin{array}{l}\text { 2. } \lambda \dot{u} \theta \eta \tau \iota \\ \text { 3. } \lambda u \theta \dot{\eta} \tau \omega\end{array}\right.$
P. $\left\{\begin{array}{l}\text { 2. } \lambda u ́ \theta \eta \tau \varepsilon \\ \text { 3. } \lambda u \theta \dot{\eta} \tau \omega \sigma \alpha \nu .\end{array}\right.$

INF. $\lambda u \theta \tilde{\eta} v \alpha ! \quad[\lambda u \theta \dot{\eta} \sigma \varepsilon \sigma \theta \alpha i]$

PART. $\lambda u \theta \varepsilon i \varsigma,-\varepsilon i \sigma \alpha,-\varepsilon \nu \quad \lambda u \theta \eta \sigma \delta \mu \varepsilon \nu \circ \varsigma$ 
§20. $x \dot{\theta} \theta \eta \mu \alpha \iota$ ( $\left(x \alpha \tau \alpha+\dot{\eta} \mu \alpha_{l}\right)$, I sit, I am seated.

STEM $\dot{\eta} \sigma-$

\section{Present Imperfect}

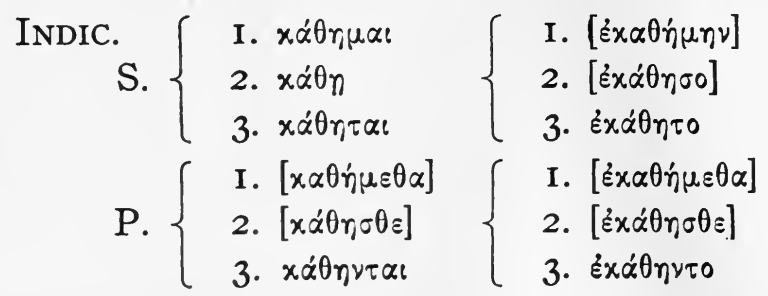

SUBJ.
S. $\left\{\begin{array}{l}\text { I. }[x \alpha \theta \bar{\omega} \mu \alpha \ell] \\ \text { 2. }[x \alpha \theta \bar{n}] \\ \text { 3. }[x \alpha \theta \bar{\eta} \tau \alpha l]\end{array}\right.$
P. $\left\{\begin{array}{l}\text { I. }[x \alpha \theta \dot{\omega} \mu \varepsilon \theta \alpha] \\ \text { 2. } x \alpha \theta \bar{\eta} \sigma \theta \varepsilon \\ \text { 3. }[x \alpha \theta \bar{\omega} \nu \tau \alpha \iota]\end{array}\right.$

IMP.

$$
\text { S. }\left\{\begin{array}{l}
\text { 2. } x \alpha^{\prime} \theta 0 u \text { (as if from } \\
\text { 3. } x \alpha^{\prime} \theta \circ \mu \alpha \text { ) }
\end{array}\right.
$$

P. \{

3.

INF.

$x \alpha \theta \tilde{n} \sigma \theta \alpha i$

PART. $\quad x \alpha \theta \dot{n} \mu \varepsilon v \circ s,-\eta,-o r$

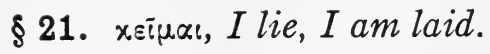
STEM $x \varepsilon t-$
Present
Imperfect
INDIC.
I. $x \varepsilon i \tilde{\mu} \alpha \mathrm{s}$
2. $[x \in \bar{i} \sigma \alpha t]$
3. $x \in i \tau \alpha i$

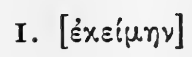
2. ["x $x \in 100]$
3. हैxะเข० 


\section{PARADIGMS OF VERBS}

Present

P. $\left\{\begin{array}{l}\text { I. } x \varepsilon i \mu \varepsilon \theta \alpha \\ \text { 2. }[x \varepsilon i \sigma \theta \varepsilon] \\ \text { 3. } x \varepsilon i \nu \tau \alpha \iota\end{array}\right.$
Imperfect
$\int$ I. $[\varepsilon x \varepsilon i f \mu \varepsilon \theta \alpha]$
2. $[$ हैx $\varepsilon เ \sigma \theta \varepsilon]$
3. Ëxยเขน०

INF. $x \in i \sigma \theta \alpha \iota$

PART.

$$
x \in i ́ \mu \varepsilon v \circ \varsigma,-\eta,-\circ \nu
$$

§22. Eijul, I am going, occurs only in compounds in the New Testament.

$$
\text { STEM i-, } \varepsilon i-
$$

$$
\text { Present Imperfect }
$$

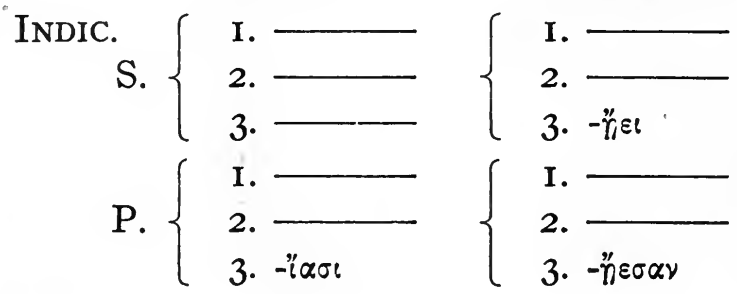

INF.

PART. $-i \varepsilon ́ \vee \alpha \ell$

$-i \dot{\omega} \nu,-i 0 \tilde{\sigma} \sigma \alpha,-i 6 \nu$. 


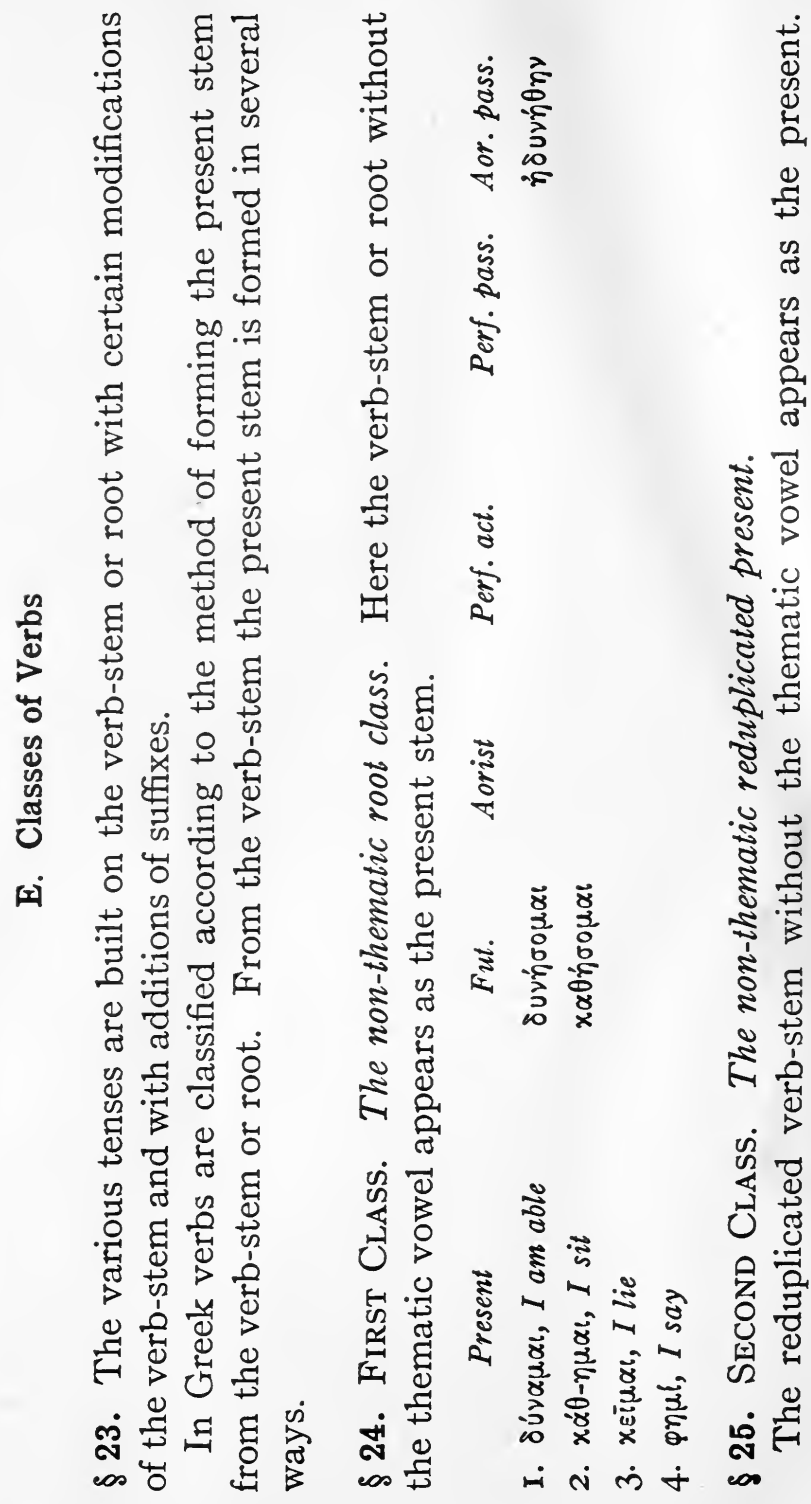




\section{CLASSES OF VERBS}

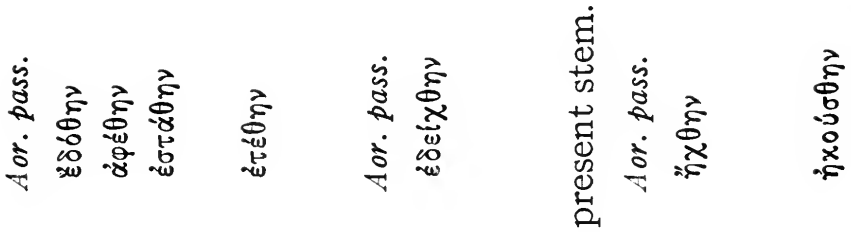

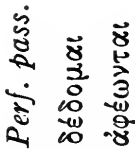

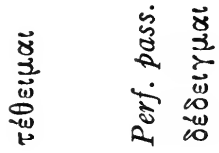

至

द्व

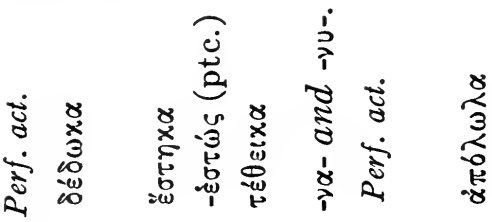

웅

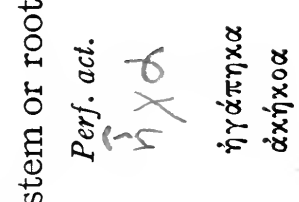

造䓎

$\frac{\sqrt{2}}{\sqrt{4}}$

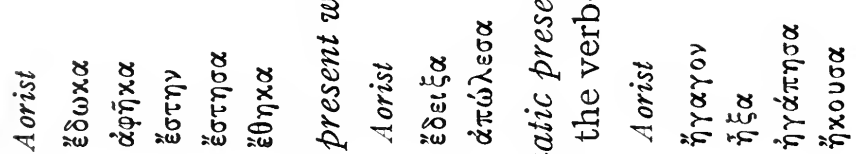

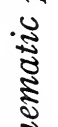

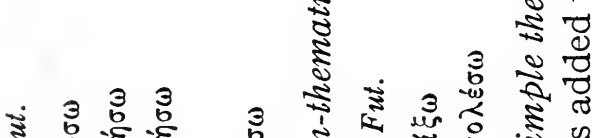

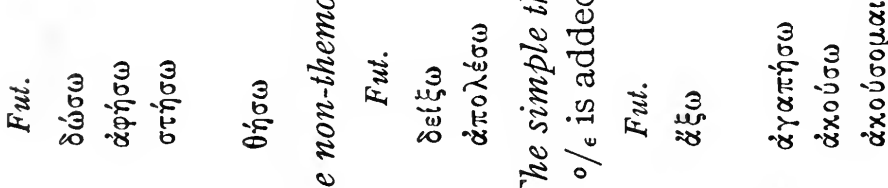

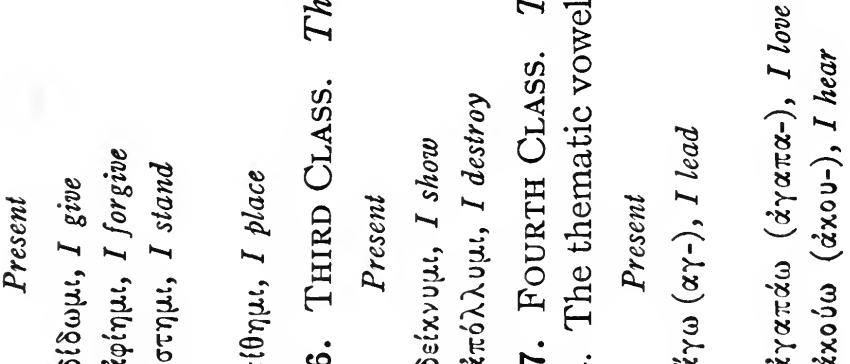

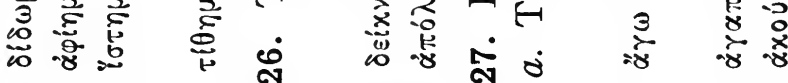

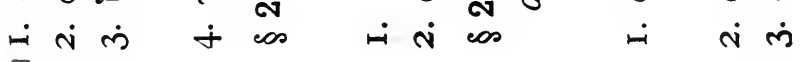


岁

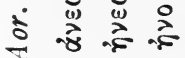

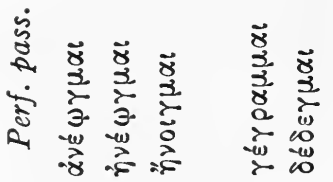

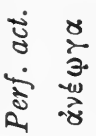

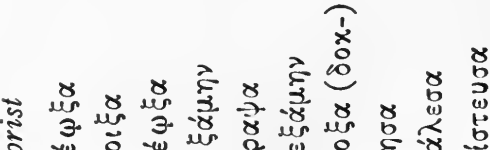

ব)

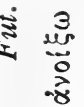

离

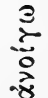

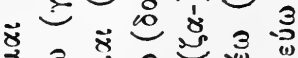

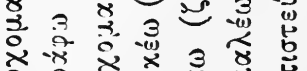

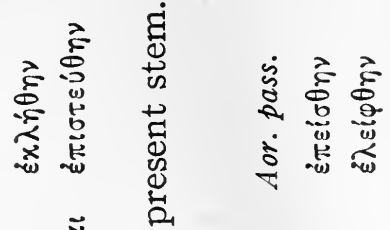

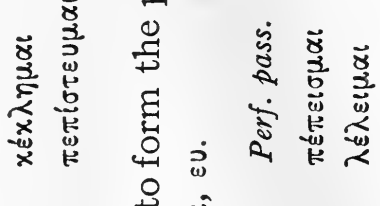

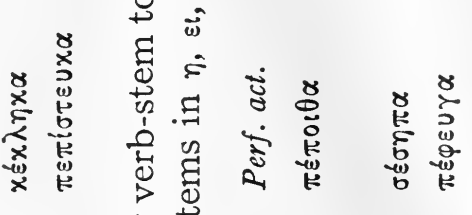

$\infty$

党宫

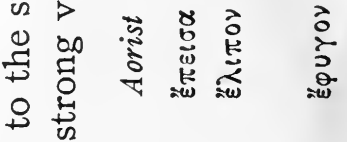

ग

రृ

垔

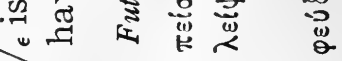

$\circ$;

$\overrightarrow{0}=$

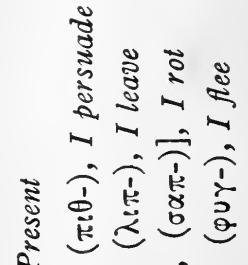

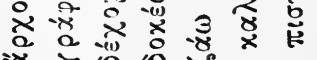

$+$

is 


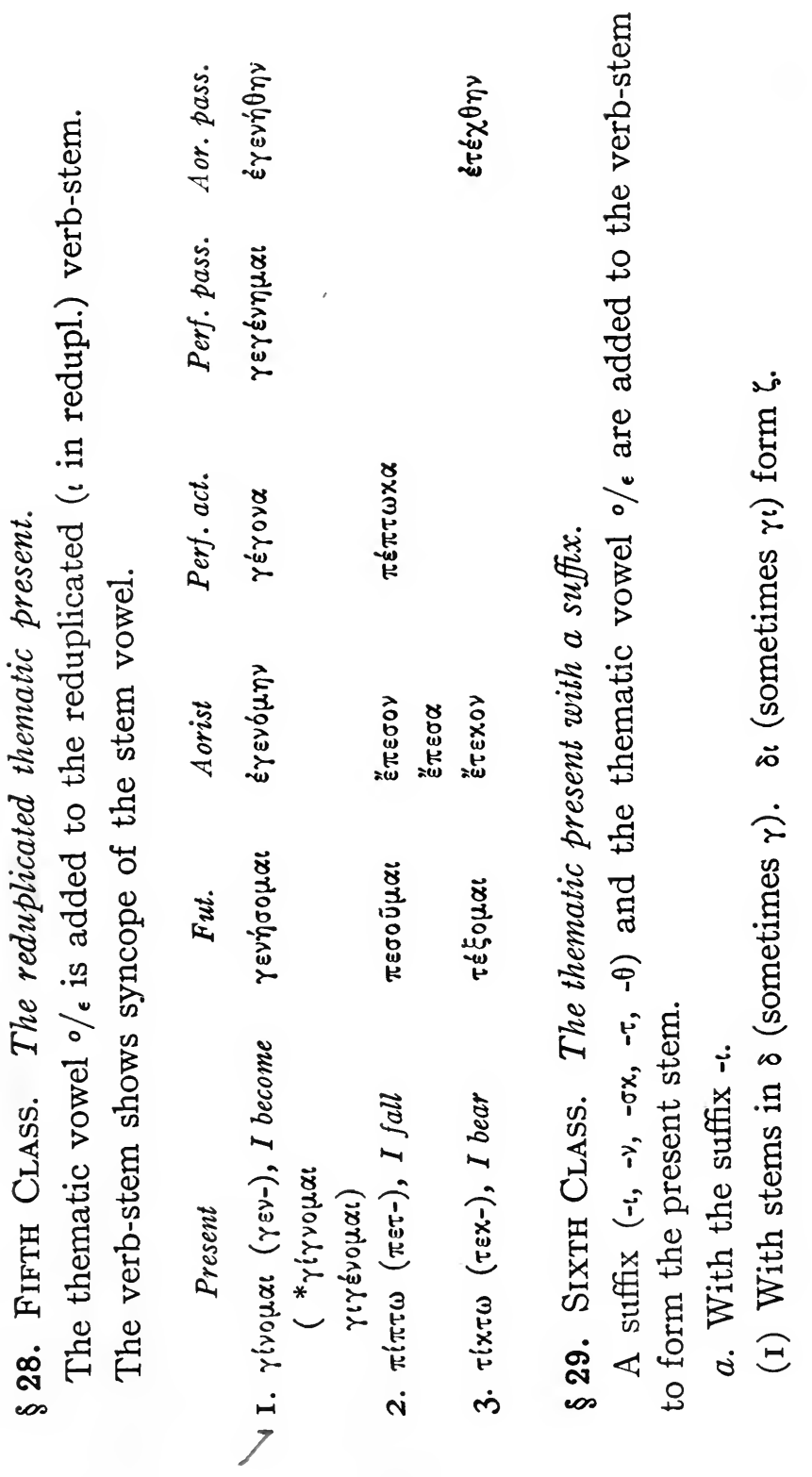




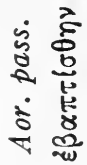

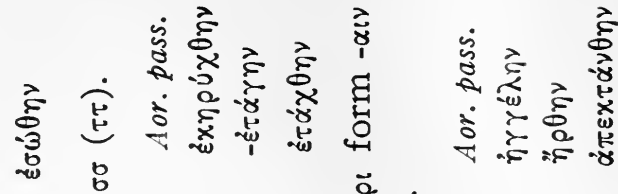

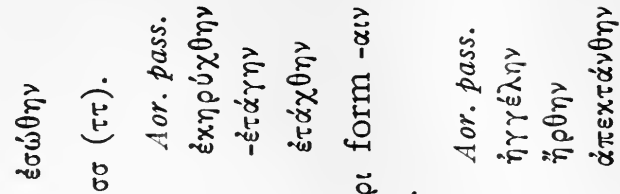

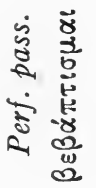

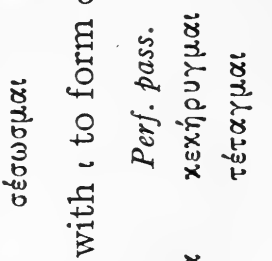

훙

च

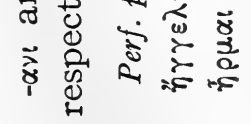

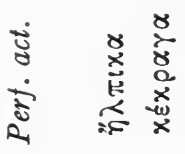

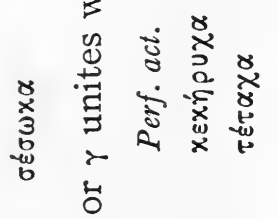

¿ 12

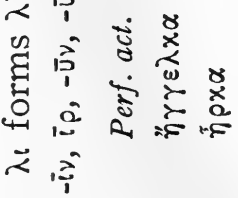

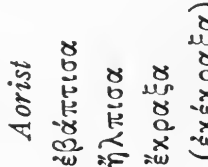

ชิ

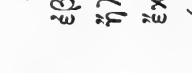

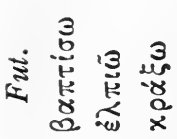

离

वे $\hat{x}$ रे

व $\hat{x}$ ये

8

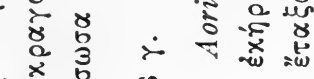

a

兽

(1)

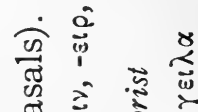

疍 党

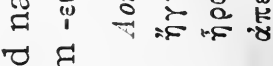

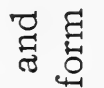

ก

is

¿

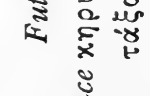

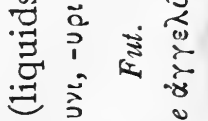

司

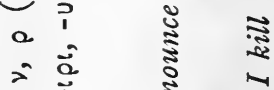

त है हो

$$
\text { co }
$$

点它㸃

ङ

$2 \sqrt[3]{3} 3$

点苋

范

- $\dot{\sim} \dot{~}$

点

7

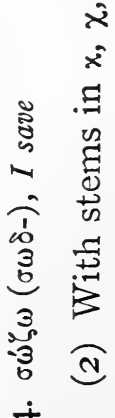

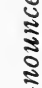

竎

2

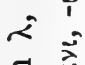

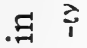

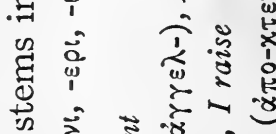

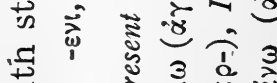

5. 35

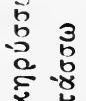

म

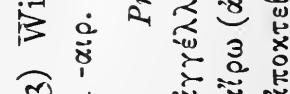

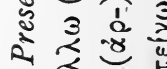

ल)

म $\dot{\text { ते }}$ 


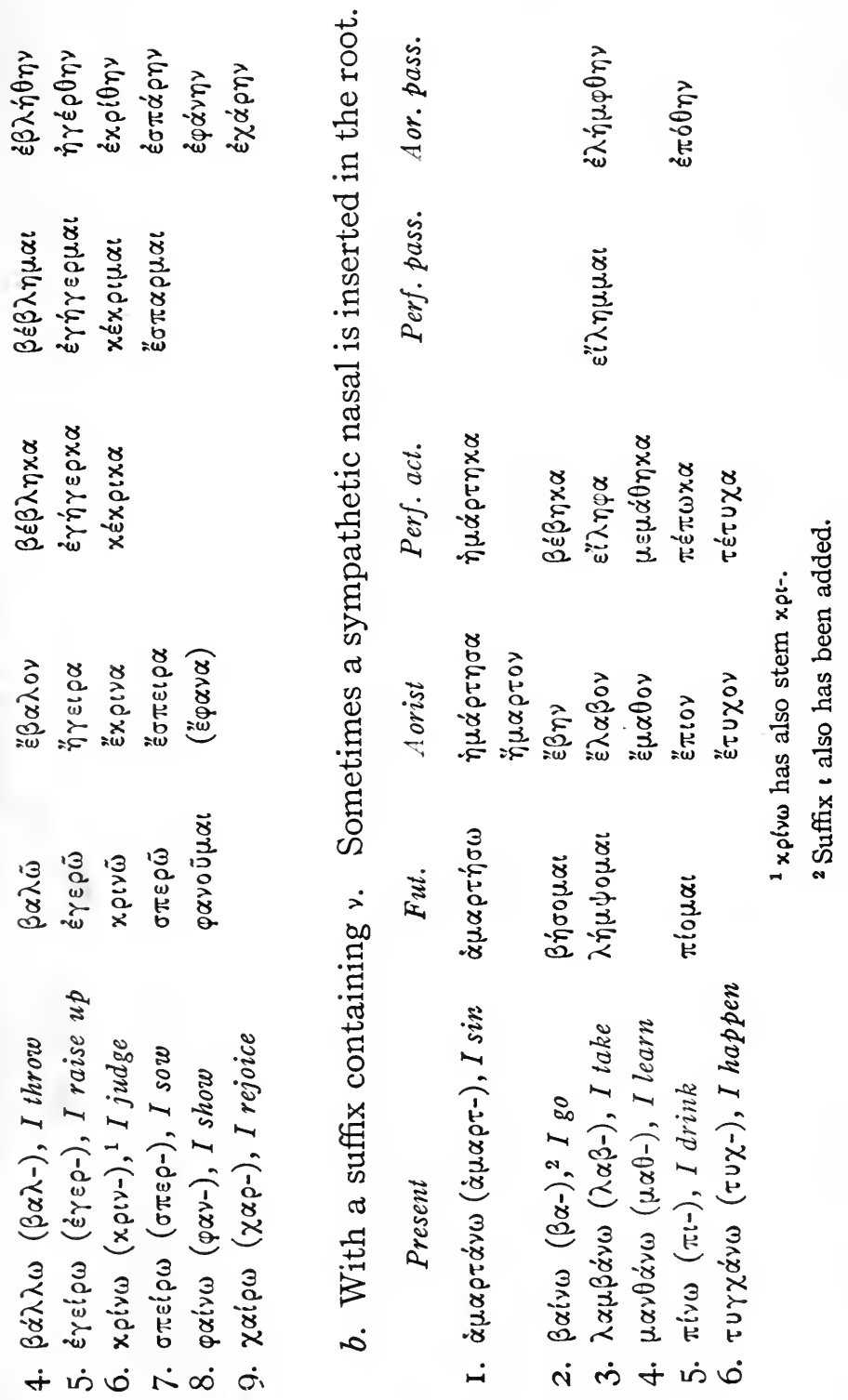




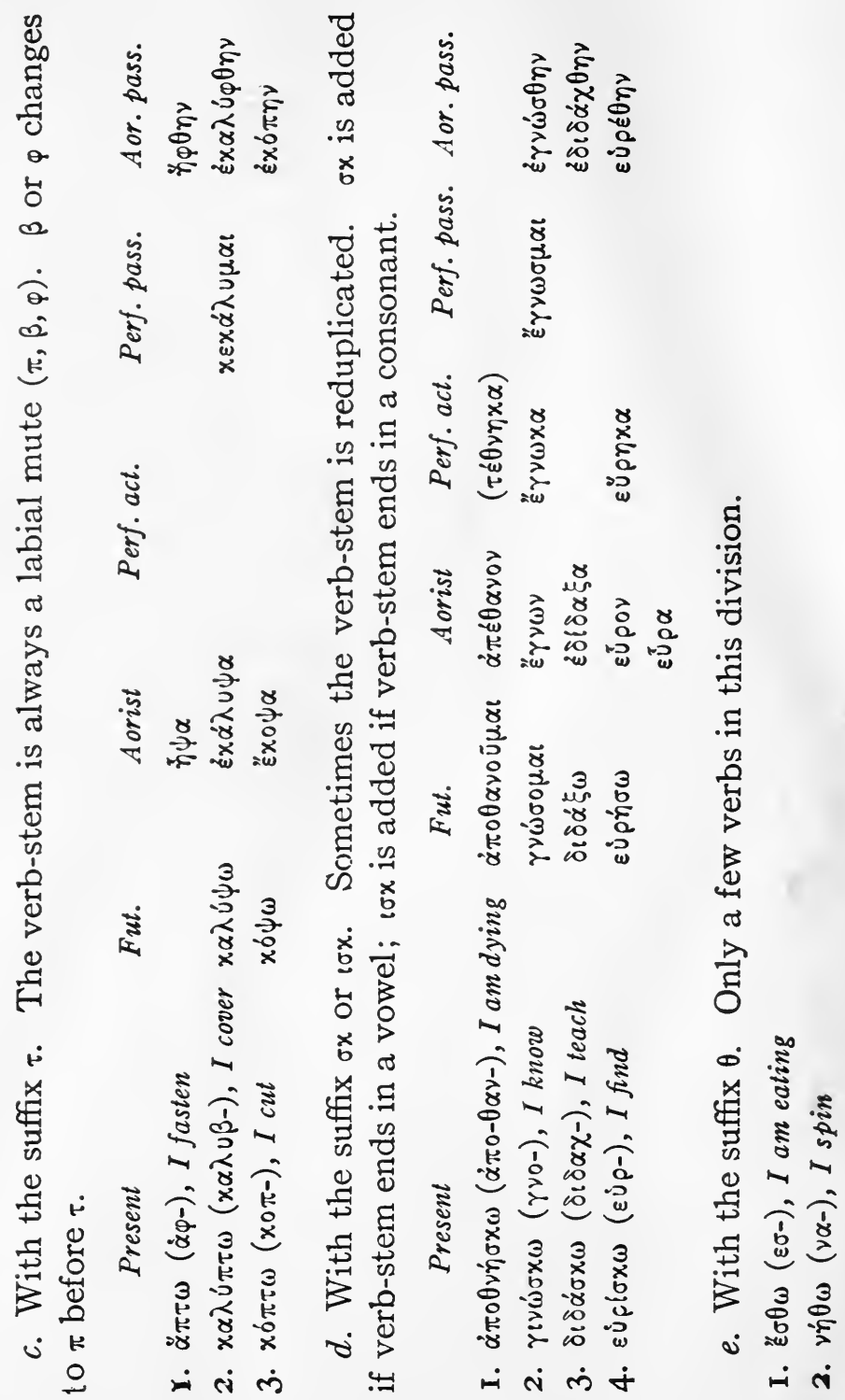




\section{CLASSES OF VERBS}

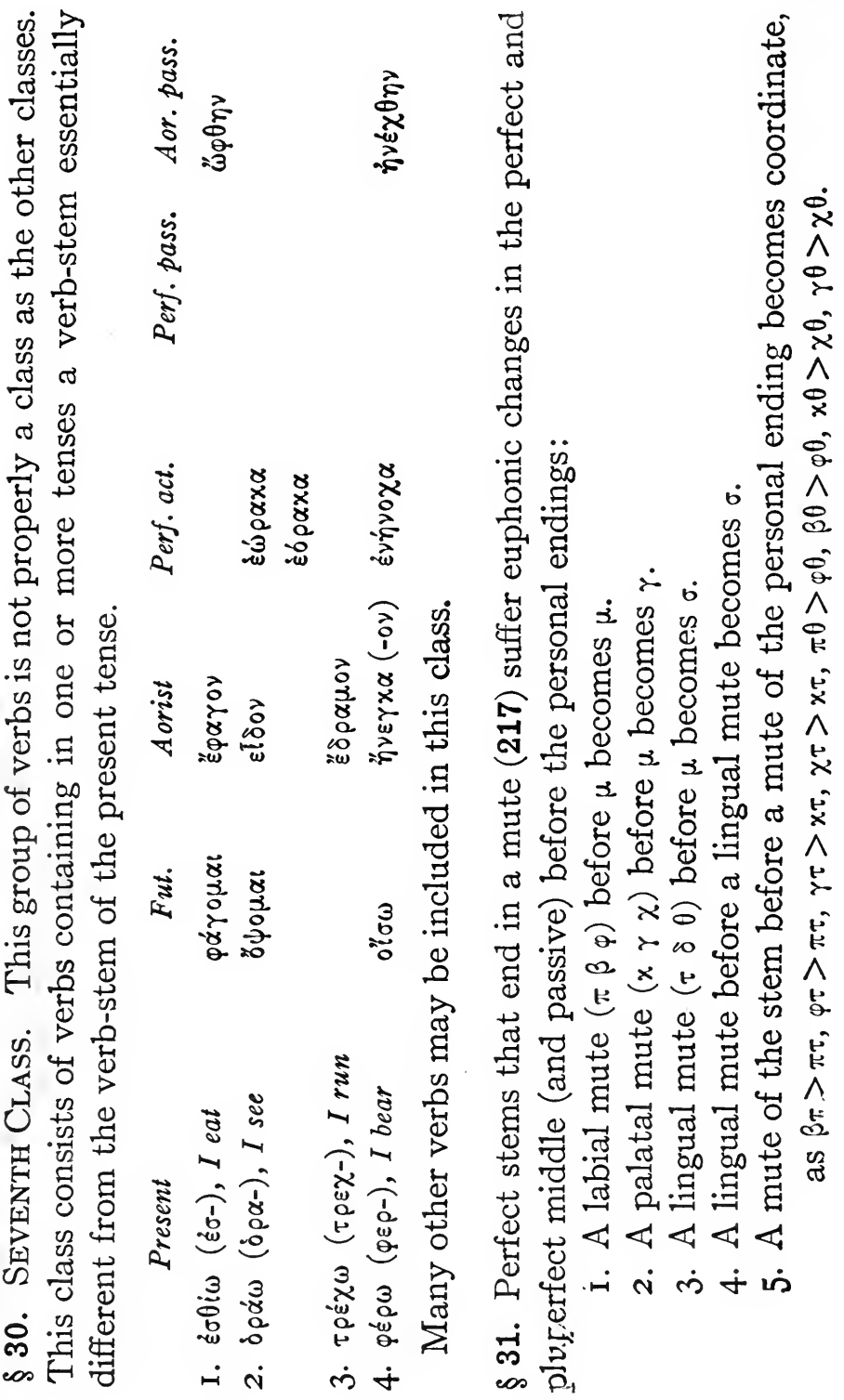


్ㅓㅇ

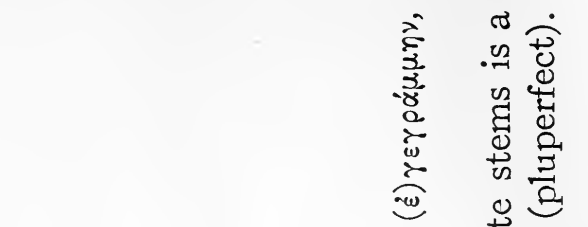

总哭节高

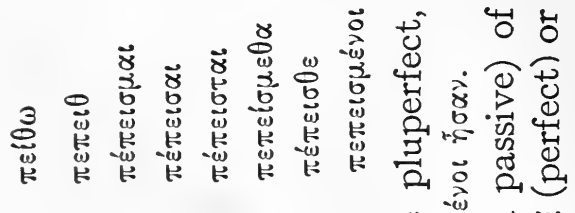

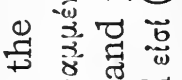

ช

$\exists$ ¿0

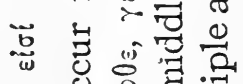

片

范

荡 券

है

0 .

点

4 $\stackrel{\omega}{x}$ م

-

की

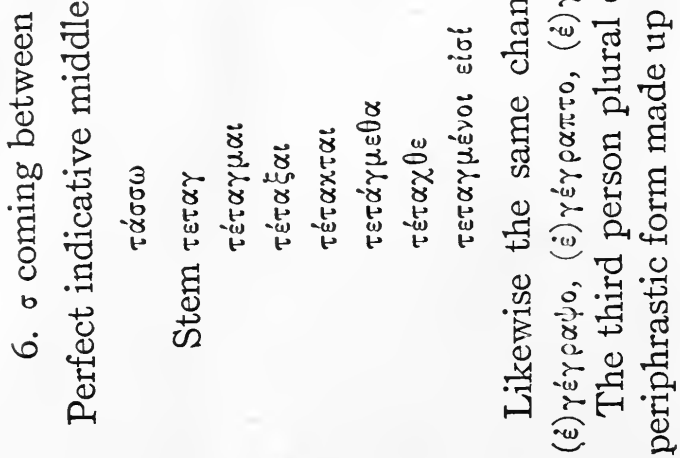

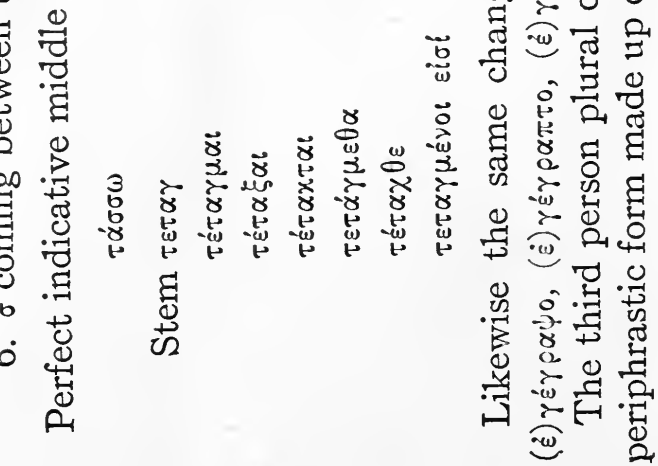


ENGLISH INDEX 


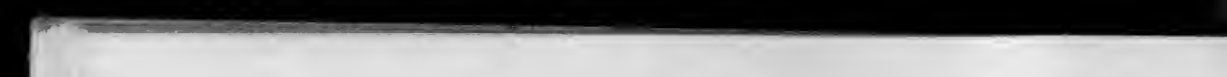




\section{ENGLISH 'INDEX ${ }^{1}$}

A

Ablative case: meaning, 29; many examples

Accent: kinds, 22; meaning, 22; where and why placed, 23 f.; recessive, 23; in declensions, 29; oxytones, 50; proclitics, 44, 50; enclitics, 63 f.; $2 \mathrm{~d}$ Aorist Inf., 79; participles, 98; comparatives and superlatives, I79; contract verbs, I $78 \mathrm{f}$.

Accusative case: meaning, 29; general reference, 132; many examples

Action: expression of, 24, 73; kind of, 25, 82, 158; durative, 25, 4I, 78, etc.; linear, 25, 4I, etc.; punctiliar, 78, etc.; Aorist, 78; participle, 99, 103, 104, I 56 f.; imperfect, 4I, 168; perfective, I 52, I 56; constative, ingressive, effective, I 24

Active voice: meaning, 36; examples, 24-27, 62 f., I 75, 73-5, $96-8$, I67, I76; 4I-3,68; 77-8I, I63, 8I-3, гог-5;86-93, I62 f.; I 20-5, I25-7, I28-30, I69; I49-52, I 54 f.; I 52 f.; I 53 ; I 55

Acute accent, 22, 31, 32, 65; many examples

Adjectives: gender, number, case, 56; agree with substantive, 32, 57; attributive and predicate use, $57 \mathrm{f}$; substituted by phrase and adverb, 59; ist declension,-endings, 56, 59; áratós, 56; forms, 56; accent, 57; 3d declension,- $-\pi \tilde{a} s$, r30; accent,

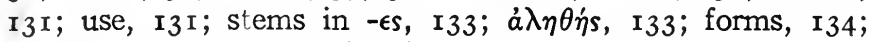

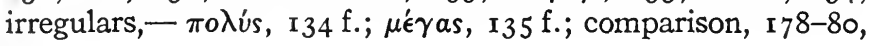
I 82

Adverbs: formation, I80; comparison, I80 f., I84

Affirmation, 24, 74, 21 2

Agent, 48

Agreement: adjectives, 32 ; pronouns, 67

Aktionsart: I 23 f., I 25, I27, I30, I 39 f., I 52, I 56, I69

Alphabet, ig $\mathrm{f}$.

Antecedent, 67, I 2

Antepenult, 23; many examples

1 All references are made to pages. 


\section{4 BEGINNER'S GREEK GRAMMAR}

Aorist: First - Ind. Act. and Mid., I20-5; Subj. Act. and Mid., I 25-8; Participle Act. and Mid., I28-30; Ind. and Subj. Pass., I38-43; Pass. Participle, I 43

Second - Ind. Act. and Mid., 77-8I, I98 f.; Subj. Act. and Mid., 8I-3; Pass., I4I

Apodosis in conditional sentence: First Class, 68; Second Class, I 57 f.; Third Class, 88; Fourth Class, 214

Article, 30 f., 32, 33, 34, 49, 50

Aspiration, 217

Attributive use: adjectives, 57, 58, I3I; participles, roo, I03

Augment, 42, 47, 79, I II, I39, I 53

B

Breathing, 21, 23; many examples

C

Cardinals, $\mathbf{7} 72$

Cases: names and meaning, 29; endings, 30; with verbs, $76 \mathrm{f}$, $5 \mathrm{I}, 56$; of infinitive, 148

Case-endings, 30,84

Causal use of infinitives, 148

Circumflex accent: $22,32,33,35 \mathrm{f} ., 50,54,74$; many examples

Circumstantial participle, 105, 108

Commands, 170

Comparative degree: adjectives, I79f., I82-4; adverbs, I80 f.

Comparison of adjectives and adverbs, I $78-84$

Comparison, standard of, I8o

Compensatory vowel lengthening, 26, 95

Complement, Infinitive as, 60

Compound verbs, 44,70

Conditional sentence: First Class, 68; Second Class, $157 \mathrm{f}$; Third Class, 88; Fourth Class; 214

Conjugation: meaning, 25; see examples and paradigms

Connective, 63

Consonant declension, 84

Constative action, $\mathrm{I} 24$

Contract verbs, 90 ; present system of $-a \dot{\omega} \omega$, I85-9, $-6 \omega$, I92-4. $-\dot{\epsilon} \omega, \mathbf{I} 74-8$ 
Contraction, $90 \mathrm{f} ., \mathrm{I} 63, \mathrm{r} 75, \mathrm{r} 78, \mathrm{r} 88, \mathrm{I} 94$

Copula, 63,70

Crasis, 217

Dative case: meaning, 29; many examples

Declension, accent in, 29

Declensions:

(a) Substantives:

First (a-stems), 49-55: gender, 49, 5I ; examples, 49, $52,53,54,55$; forms explained, 49 f., 52 f.; accent, 50 , 54 ; contracts, 54

Second (o- stems), 27-36: stems, 28; accent, 28, 29, 3 If., 33 ; examples, 28,3 I, 33 f.; forms explained, 28 f., $3 \mathrm{I}, 33,34$; gender, 30

Third (consonantstems): neuters in - $\mu \alpha \tau-, 83-6$, - name, 84 ; endings, 84 ; forms explained, 84 f.; gender, 85 ; stem, 85 f.; examples, 85; Lingual Mutes, 93-96,- stem, 93; forms explained, $94 \mathrm{f}$.; accent, $94 \mathrm{f}$.; examples, $94 \mathrm{f}$.; Mutes and Liquids, ro6-9, - examples, $106 \mathrm{f}$.; forms explained, I07 f.; labial mutes, I07; Liquids in - $\epsilon \rho$ (syncopated), r ro f.,- examples, I го; forms explained, I го; Stems in ᄂ, II3 f., - examples, II4; forms explained, II4; Stems in $-\epsilon \nu$ and $-\epsilon s$, II 7-20, - examples, II 8 f.; forms explained, II8f.; gender, II8; accent, II 9 ; neuters in $-o$, I 19 ; Irregulars, 146

(b) Adjectives:

First and Second, 56-60

Third, I 33 f.

Irregular, $\mathrm{I}_{34}-6$

Comparative degree, 183

(c) Pronouns:

Personal: Ist person, 6I; 2d person, 6I; 3d person, 66 Demonstrative, $7 \mathrm{I}$

(d) Participles, 97 f., I02, I43, I 54, I77, I87 f.

(e) Numerals, I7 I-3

Defective (deponent) verbs, $69 \mathrm{f}$.

Demonstrative pronouns, 7 I f., I8I

Diaeresis, 217

Digamma, I I 8, footnote

Diphthongs, 2 I 
Discourse, Indirect, $136 \mathrm{f}$.

Dissyllabic Enclitic, 64

Durative action, 25, 4I, 75, 78, 80, 82, 87, 99, 103, 123, I68

$\mathrm{E}$

Effective action, I 24

Elative sense of superlative, 180

Elision, 217

Emphasis, 62

Enclitics, 62, 63, 64 f., II 6

Endings; verbs, see personal endings; cases, see case endings

Entreaties, 170

Exercises, English and Greek: see each lesson

Exhortations, 76, I 70

Feminine gender, 49, 5I f.; many examples

First ( $a$ - stems) declension: see declensions

Formative vowel lengthening, 95, I 10

Future tense: Ind. Act. and Mid., 83-93; Pass. Ind., I40 f.; Ind. Act. and Mid. of liquid stems, I62 f.; Sec. Future Pass., I4I; see paradigms

\section{G}

Gender, 49, 51, 52, 56, 86; many examples

Genitive absolute, $108 \mathrm{f}$.

Genitive case: meaning, 29; many examples

Gradation of vowels, 218

Grave accent, 22, 31; many examples

$\mathrm{H}$

Hesitating affirmation, 212

Historical tenses, 42 ; see paradigms

Identical pronoun, 66

\section{I}

Imperative mode: development, r66; personal endings, 166 , I68; how formed, $\mathrm{I}_{7}$; Pres. and Aor. compared, 168 ; kind of action, I68 f.; Aktionsart, r69 (see Aktionsart); significance, I70; negative, I70; persons, I70; personal endings, I66; examples, $167-70$; see paradigms 
Imperfect tense: stem, $4 \mathrm{I}$; kind of action, $4 \mathrm{I}$; personal endings, 4I, 46, see personal endings; thematic vowel, 42, 47, see thematic vowel; augment, $42 \mathrm{f}$., 47 , see augment; examples, $4 \mathrm{I}-43,46 \mathrm{f} ., 68$, I 76 ; see paradigms

Impersonal verbs, $\mathrm{I} 8 \mathrm{~g} \mathrm{f}$.

Indefinite pronoun, Ir 5-7

Indefinite relative pronoun, $16 \mathrm{I}$

Indicative mode: Present, 24-27, 36-40, 62 f., I75; Imperfect, 4I-3, 46 f., 68, I76; Future, 86-93, I40-2, I45, I62 f.; Second Future, I4I; Aorist, I 20-5, I38 f., I45, I'63; Second Aorist, 77-8I, I4I, I44, I63; Perfect, I49-52, I 55 f.; Pluperfect, I 52 f., r56; Second Pluperfect, I52f. See paradigms

Indirect discourse: assertions, $136 \mathrm{f}$; questions, 136 ; commands, I 37

Infinitive: no personal endings, $27,132,149$; as complement, 60; action of, $79 \mathrm{f}$; future, 87 footnote; voice and tense but not mode, I46; verbal substantive, I $46 \mathrm{f}$; with neuter article, I 47; purpose, I $47 \mathrm{f}$; in substantive constructions, I $47 \mathrm{f}$; temporal, I48 f.; causal, I48; time, I49; tense, I49; no augment, 79; no subject, I32, I47; case, I 48 f.; negative, I48; examples, see paradigms

Ingressive action, 124,169

Instrumental case: meaning, 29; many examples

Intensive pronoun, 66

Interchange of vowels, 218

Interrogative pronoun, II $5-7$

Iota-subscript, 21, 28, 49, 74, 76; many examples

$\mathrm{K}$

Koiné, I9

\section{$\mathrm{L}$}

Labial mutes, 9I, I07, I23, I42

Linear action, 25, 4I, 75, 78, 82, I 23

Lingual mutes, 9 I, $93-6$, I 23, I 5 I, I 56

Lingual mute stems, 93-6

Liquids, I06-8, I 10 f., I42, I 5 I, I56, I62-5

Locative case: meaning, 29; many examples 
M

Masculine gender, 30, 5I f., 54; many examples

$\mu \iota$ - verbs: $\omega$ - and $\mu \iota$-conjugations, 63 , I95 f.; examples, I96-9, I99, $20 \mathrm{I}-3,203 \mathrm{f}$, 205-8, $209 \mathrm{f}$; ; compounds, I 96 footnote

Middle voice: meaning, $36 \mathrm{f}$; primary personal endings, 37, 40; thematic vowel and personal endings, 38; examples, 36-9, $46 \mathrm{f} ., 75-7,77-8$ I, 8I-3, 86-93, 98 f., I 20-5, I25-30, I 55 f.; I67-9, I 75 f.; see paradigms

Monosyllables, 94

Monosyllabic Enclitic, 64

Mode, 24-73; see indicative, subjunctive, imperative, optative; see paradigms

Movable $\nu, 65$ footnote

Mutes, 9I f., 93-6, I06-8, I23, I42, I 5 I, I 56

Nasal stems, I07, I 50

N

Negatives: in conditional sentence, 68, I58; with participle, Ior; with infinitive, I48; with imperative, I70; in ordinary sentence, I73; in direct questions, 173

Neuter gender, 5I f., I I9; many examples

Nominative case: meaning, 29; many examples

Number, 48, 5r; many examples

Numerals: list, I 7 I ; declension, I 7 I-3

Old forms of verbs, 2 Io $f$.

$\mathrm{O}$

Optative mode: meaning, 2 г2; N. T. use, 2r 3 ; examples, 2 I 3 f., see paradigms; wish about present, past, future, 2 I4; fourth class condition, 2 I 4

Oxytones, 50

$\omega$ conjugation, 63; verbs, 195

Palatals, 9 I, I 23, I42

$\mathrm{P}$

Paradigms:

Nouns: (a) Adjectives. - $a$ - and o-declension, 22 I f.; $3 \mathrm{~d}$ de-

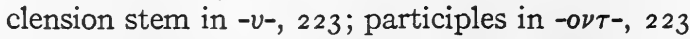

(b) Substantives - Masculines in -as of Ist decl., 219; 
$3 \mathrm{~d}$ declension stems in $-v-, 2 \mathrm{Ig} \mathrm{f}$., in $-0 v-, 220$; some peculiar forms, $220 \mathrm{f}$.

Verbs: $\lambda \dot{v} \omega, 225-9 ; \kappa \dot{\theta} \theta \eta \mu a \iota, 230 ; \kappa \epsilon i \tilde{\mu} a \iota, 230$ f.; $\epsilon \tilde{i} \mu \iota, 23 \mathrm{I}$

Participles: gender, 97; accent, 98; verbal adj., 99; time, 99; tense, 99; no personal endings and mode, roo; attributive use, IOO, I03; negative, I0I; position, I04; declension, 97 f., I02, I43, I 54, I77, I87 f.; formation, 99; endings, IoI, I 56; predicate use, I03; action, I04, I 56; Aktionsart, I30, I56; circumstantial, ro5; examples, $98 \mathrm{f}$., 96-Ior, 105-5, I28-30, $143 \mathrm{f}$., I54 f., 156

Passive voice: meaning, 36 ; form, 39 , I40; examples, $39 \mathrm{f}$., 46 f., $75-7,98$ f., I 38 f., I 40 f., I $42-5$, I 55 f., I67, I69 f., I 75 f.; see paradigms

Penult, 23; many examples

Perfect tense: meaning, I 52 ; formation, $\mathrm{I} 50 \mathrm{f}$., I $55 \mathrm{f}$.; Aktionsart, I 52 ; examples, I49-52, I 54-6; see paradigms

Perfective: action, I 52, I 56; force, 45

Person, 48

Personal endings: meaning, 24,48

(a) Primary - 26, 37, 38, 40, 74, 76, 82, 88, I20, I26, I39, I40, I 55

(b) Secondary - 4I, 46, 79, I20, I21, I39, I56

Personal endings of imperative, 166

Personal pronouns, 60-2; 65-7

Pluperfect tense: formation, I 53 ; meaning, 153 ; examples, $152 \mathrm{f}$., I 56 ; second, I 53; see paradigms

Plural subject with singular verb, 86

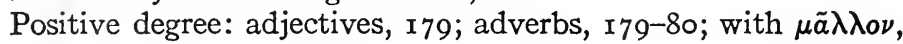
I8r

Postpositives, 6I

Predicate nominative, $6_{3}$

Predicate position: adjectives, $57 \mathrm{f}$., I3I; participles, I03

Prepositions: meaning, 44; proclitics, 44 ; with cases, 44 f.; in compound verbs, 45 ; final vowel dropped, 45

Present tense, 24-27, 62 f., 73-5, 75-7, I67, I 75 f.; see paradigms

Present system of contract verbs: in $-\dot{\epsilon} \omega, 174-8$; in $-\dot{a} \omega, 185-9$; in $-o ́ \omega, 19 I-5$

Primary personal endings: see personal endings]

Primary tenses, 26 footnote, 46 footnote

Principal parts of verb, 165 ; see paradigms

Proclitics, 44, 50, 65 
Prohibitions, I27, I70

Pronoun: meaning, 6r; use, 6r ; personal, 60-2; 65-7; identical, 66 ; intensive, 66; interrogative, I I 5 f.; indefinite, I I 5-7; relative, II If., I6I; reciprocal, I60; reflexive, I58-60; indefinite relative, I6r; demonstrative, $66,7 \mathrm{I}$ f., 224

Protasis: first class condition, 68; second class, $\mathrm{I}_{57} \mathrm{f}$.; third class, 88 ; fourth class, $2 \mathrm{I} 4$

Punctiliar action, $78,79,82,87,99$, I03, I23, I24, I27, I39 f., I 68

Punctuation, 218

Purpose: clauses of, 74 ; with infinitive, $147 \mathrm{f}$.

\section{Q}

Questions: direct, I16; negative, I73; 1ndirect, I16; doubt, 8 :

Question mark, 75 footnote

$\mathrm{R}$

Recessive accent, 23, $178 \mathrm{f}$.

Reciprocal pronoun, 160

Reduplication, $\mathrm{I} 50 \mathrm{f}$., $\mathrm{I} 55$

Reflexive pronoun, $158-60$

Relative pronoun: antecedent, III f.; attraction, II 2; declension, III

Result: $\omega \sigma \tau \epsilon$ with infinitive, $\mathbf{1} 32$

Rough breathing, 21, 33; many examples

$\mathrm{S}$

Second declension, 27-36; see declensions

Secondary personal endings: see personal endings

Secondary tenses, 26 footnote, 42, 46 footnote

Sounds and writings, 2 I $7 \mathrm{f}$.

Strong perfects, I 5 I

Stem: meaning; (a) Substantives - 85, 93-6, 107; (b) Verbs $25 \mathrm{f} ., 4 \mathrm{I}, 80,87,92,93, \mathrm{I}_{2}$, I $5 \circ \mathrm{f}$., I $55 \mathrm{f}$.

Stem, Thematic, 80 , Ior f.; see thematic vowel

Stress of voice, 22

Subjunctive mode: meaning, 74 ; tense, 74 ; clauses of purpose, 74 ; mode sign, 74,76 ; personal endings, 74,76 ; negative, 75 ; action, 75; time, 75 ; examples, $73-5,75-7,81-3$, I 25-7, I39, I 75; see paradigms 


\section{ENGLISH INDEX}

Suffix, 88,90

Superlative degree, $179 \mathrm{f}$.

Supplement to Part I, 21 7-39

Syllables, 20

Syllabic augment, 42

Syncope, 217

Temporal use of infinitive, 148

Temporal augment, 42

Tense: meaning, 25,73 ; systems, I64f.; see paradigms

Thematic stem, 8o, IoI f.

Thematic vowel, $25,26,38,42,47,74,76,80,82,88,96,99$, I26, I39, I40, I 53, I62, I68

Third declension: see declensions

Time, 25, 82, 96; see various tenses

Tone of voice, 22

U

U1tima, 23, 33; many examples

Verbal adjective, 99

Verbs: personal endings, 24 (see personal endings); tense, mode, and voice, 24; primitive form, 26; classes of, 232-9; see paradigms

Vocative case: meaning, 29; numerous examples

Vocabularies: see each lesson

Voice, 36 f., 73, 48; see various conjugations

Vowels, 21; lengthening, 26, I 22, I40, I79; contraction, I63, I 88, I 94

Vowel stems, I40f., I42, I $50 \mathrm{f}$.

Writings and sounds, 2 I $7 \mathrm{f}$. 


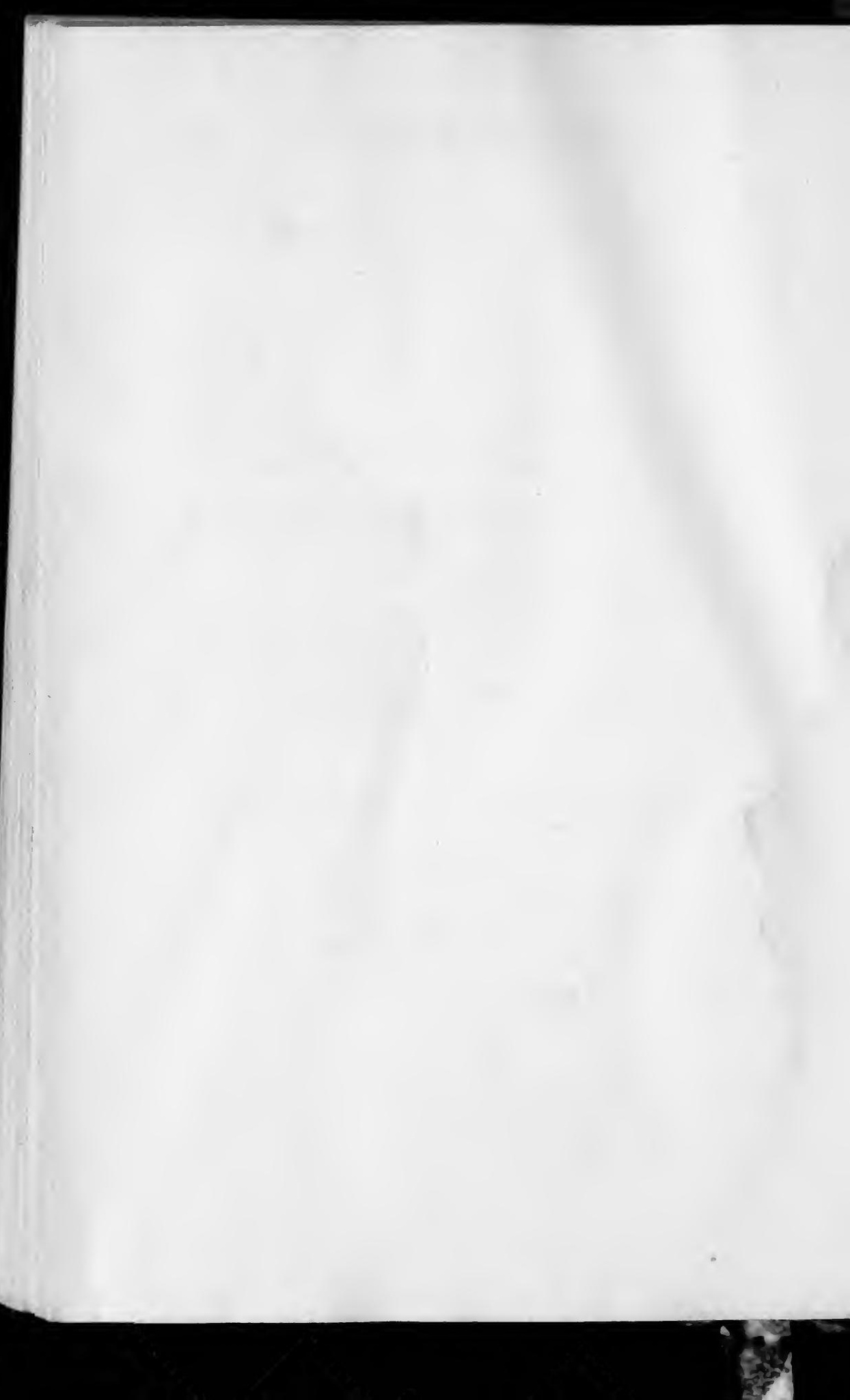




\section{VOCABULARIES}




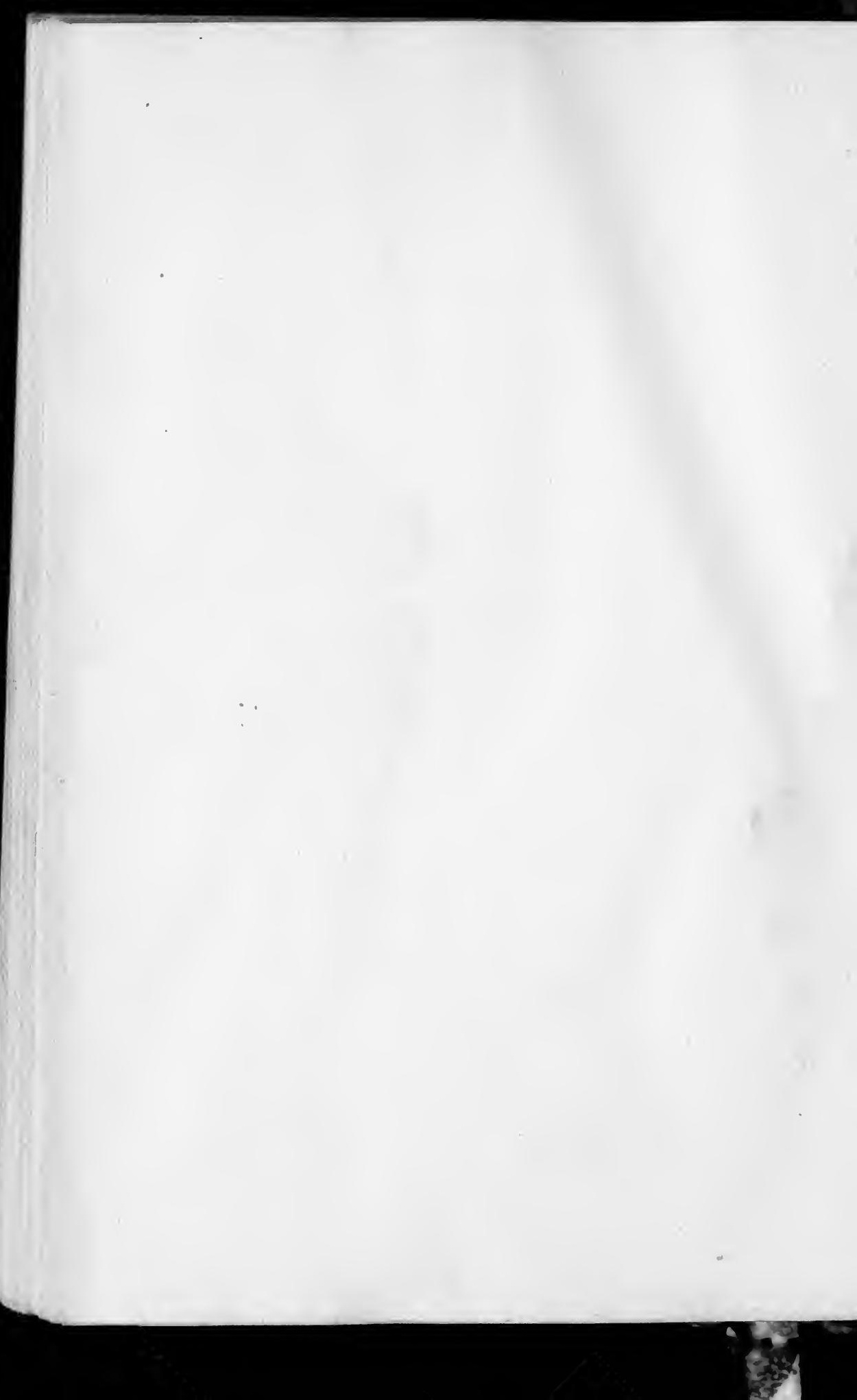




\section{GREEK-ENGLISH VOCABULARY}

A

$\dot{\alpha} \gamma \alpha \theta b \varsigma,-\eta \dot{\eta},-6 \nu$, good

$\dot{\alpha} \gamma \alpha \pi \dot{\alpha} \omega, I$ love

$\dot{\alpha} \gamma \dot{\alpha} \pi \eta,-\eta s, \dot{\eta}$, love

$\dot{\alpha} \gamma \alpha \pi \eta \tau \sigma o ́ s,-\dot{\eta},-o ́ v$, beloved

$\ddot{\alpha} \gamma \gamma \varepsilon \lambda \circ \varsigma,-0 \cup, \dot{\delta}$, messenger,

$$
\text { angel }
$$

$\dot{\alpha} \gamma(\alpha \dot{\zeta} \zeta \omega$, I sanctify

$\ddot{\alpha} \gamma 105,-\alpha,-0 \nu$, holy

$\dot{\alpha} \gamma \circ p \alpha \dot{b} \omega, I$ buy

«̋ $\gamma \omega$, I lead, bring, go; sec. aor., "irarov.

$\dot{\alpha} \delta \varepsilon \lambda \varphi^{\delta} \varsigma,-0 \tilde{u}, \dot{\delta}$, brother

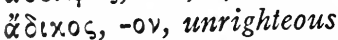

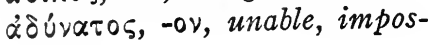
sible

$\alpha i \mu \alpha,-\alpha \tau \circ \varsigma, \tau o ́, b l o o d$

$\alpha " \rho \omega$, I take up, bear

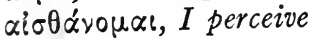

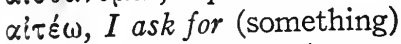

$\alpha i \dot{\omega} y,-\tilde{\omega} v o s, \dot{\delta}$, age (space of time), world

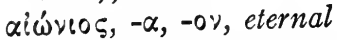

$\dot{\alpha} x \alpha \dot{\theta} \theta \rho \tau \sigma \varsigma \varsigma,-0 \nu$, unclean

$\dot{\alpha} \times 0 \lambda$ ०० $\theta \varepsilon \dot{\varepsilon} \omega, I$ follow

$\dot{\alpha} \times 0$ ú $\omega, I$ hear

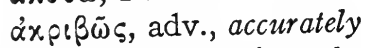

$\dot{\alpha} \lambda \dot{\varepsilon} \% \tau \omega \rho,-0 \rho \circ 5, \delta$, cock

$\dot{\alpha} \lambda \dot{r} \theta \varepsilon \iota \alpha,-\alpha \varsigma, \dot{\eta}$, truth

$\dot{\alpha} \lambda r_{i} \theta \dot{\eta} s,-\varepsilon \varepsilon_{s}$, true

$\dot{\alpha} \lambda r, \theta \in v o ́ s,-\dot{\eta},-o ́ v$, true

$\dot{\alpha} \lambda r_{i} \theta \tilde{\omega} s$, adv., truly, surely

$\dot{\alpha} \lambda \lambda \dot{\alpha}$, adversative conj., but. $\dot{\alpha} \lambda \lambda \dot{n} \lambda \omega \nu$, (gen. masc. plu.), of one another

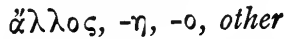

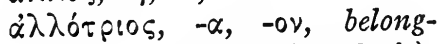
ing to another (another's),

strange

$\dot{\alpha} \mu \alpha \rho \tau \alpha \dot{\nu} \nu \omega, I \sin$

$\dot{\alpha} \mu \alpha \rho \tau i \alpha,-\alpha \varsigma, \dot{\eta}, \sin$

$\dot{\alpha} \mu \dot{n} \nu$, adv., truly, verily

$\dot{\alpha} \mu \pi \varepsilon \lambda \omega \dot{\omega} \nu,-\tilde{\omega} \nu \circ \varsigma, \dot{o}$, vineyard

$\ddot{\alpha} \nu$, see page 157

$\dot{\alpha} \vee \dot{\alpha}$, prep., on, upon, along; only used with acc. in N. T. $\alpha v \alpha \beta \alpha i v \omega, I$ go $u p$, come $u p$, ascend

$\alpha \dot{\nu} \alpha \beta \lambda \varepsilon \dot{\varepsilon} \pi \omega, I$ look up, recover sight

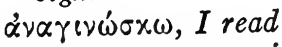

$\dot{\alpha} \vee \alpha \dot{\sigma} \tau \alpha \sigma \iota \varsigma,-\varepsilon \omega \varsigma, \dot{\eta}$, resurrection

$\ddot{\alpha} \nu \varepsilon \mu \circ \varsigma,-0 \cup, \dot{\delta}$, wind

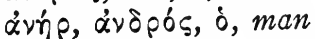

$\dot{\alpha} \nu \theta i \sigma \tau \eta \mu,, I$ set against, withstand

$\ddot{\alpha} \nu \theta p \omega \pi \circ \varsigma,-\circ \cup, \dot{o}$, man

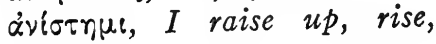
arise

¿voi $\gamma \omega, I$ open

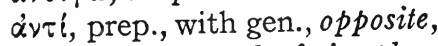
against; instead of, in place of, for

$\ddot{\alpha} \nu \omega$, adv., $u p$, above

$\ddot{\alpha} v(\omega \theta \varepsilon v$, adv., from above, again

$\ddot{\alpha} \xi \circ 0 \varsigma,-\alpha,-0 \nu$, fitting, worthy 
$\dot{\alpha} \pi \alpha \gamma \gamma \varepsilon \hat{\varepsilon} \lambda \lambda \omega, I$ announce, declare

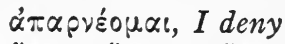

$\ddot{\alpha} \pi \alpha \varsigma, \ddot{\alpha} \pi \alpha \sigma \alpha, \ddot{\alpha} \pi \alpha \nu$, all, altogether

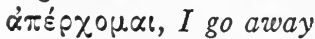

$\dot{\alpha} \pi \varepsilon ́ \chi \omega, I$ keep off, have in full (of receipts); midd., I keep myself from, abstain

$\dot{\alpha} \pi \dot{0}$, prep., from off, used only

with the abl. in the N. T.

¿ं $\pi 0 \delta 1 \delta \omega \mu$, I give up, give back,

restore; pay; midd., sell

$\dot{\alpha} \pi 0 \theta \nu \dot{r} \sigma \times \omega, I$ die

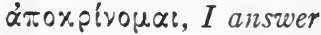

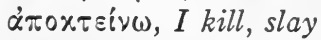

$\dot{\alpha} \pi 0 \lambda \dot{u} \omega$, I release

$\dot{\alpha} \pi 0 \sigma \tau \varepsilon \hat{\varepsilon} \lambda \omega, I$ send forth

$\dot{\alpha} \pi \delta \sigma \tau 0 \lambda \circ \varsigma,-0 \cup, \dot{\delta}$, apostle

$\ddot{\alpha} \pi \tau \omega$, I fasten to; midd., $\check{\alpha} \pi \tau-$

op $\alpha$, I touch

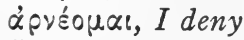

äprt, adv., now, just now, this

moment

äpros, -ou, ó, bread

$\dot{\alpha} p x \dot{\eta},-\bar{\eta} s, \dot{\eta}$, beginning

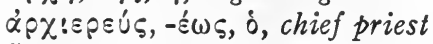

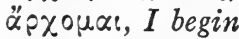

«p $\omega \nu \nu,-0 \vee \tau \circ \varsigma, \dot{\delta}$, ruler, prince

$\dot{\alpha} \theta \theta \dot{\varepsilon} v \varepsilon i \alpha,-\alpha_{\mathcal{S}}, \dot{\eta}$, weakness

$\dot{\alpha} \sigma \theta \varepsilon \nu \eta \dot{s} \varsigma,-\varepsilon ́ \varsigma$, weak, sick

aüprov, adv., tomorrow

aữós, -ฑ́, -ó, self, very, same; he, she, it

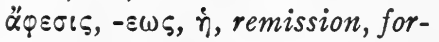
giveness

a'pinul, I send away, forgive, leave, let

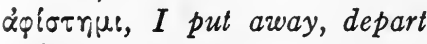
from

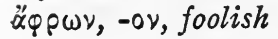

B

$\beta \alpha i v \omega, I$ go

$\beta \alpha \lambda \lambda \lambda \omega, I$ throw, cast

$\beta \alpha \pi \tau i \zeta \omega$, I baptize

$\beta \alpha \sigma i \lambda \varepsilon i \alpha,-\alpha s, \dot{\eta}$, kingdom

$\beta \alpha \sigma i \lambda \varepsilon u ́ \varsigma,-\varepsilon \dot{\omega} \omega s, \delta$, king

$\beta \alpha \sigma i \lambda \varepsilon u ́ \omega, I$ am king, I reign

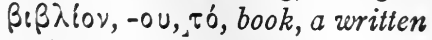
document

$\beta$ ios, $-0 \cup, \delta$, life, manner of life $\beta \lambda \varepsilon \pi \omega$, I see, look at, behold

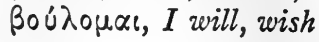

\section{$\Gamma$}

$\gamma \alpha \dot{\mu} \mu \circ \varsigma,-o u, \delta$, marriage

$\gamma \alpha \dot{p}$, coördinating conj., for.

$\gamma \hat{\varepsilon}$, enclitic, postpositive parti-

cle giving especial promi-

nence to a word, indeed, at last

$\gamma \varepsilon v \vee \alpha ́ \omega, I$ beget

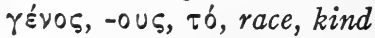

$\gamma \tilde{\eta}, \gamma \tilde{\eta} s, \dot{\eta}$, earth

rivouat, I become, be

$\gamma(\nu \omega \dot{\sigma} \sigma \omega, I$ know

$\gamma \lambda \tilde{\omega} \sigma \sigma \alpha,-\gamma, s, \dot{\eta}$, tongue

$\gamma \nu \bar{\omega} \sigma \mathrm{s},-\varepsilon \omega \varsigma, \dot{\eta}$, knowledge

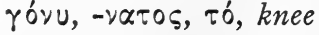

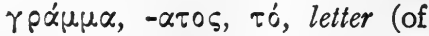

alphabet), writing

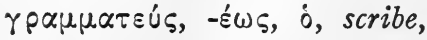
town-clerk

$\gamma \rho \alpha \varphi \dot{\eta},-\tilde{\eta} s, \dot{\eta}$, writing, scripture

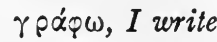

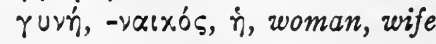

$\Delta$

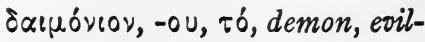
spirit 


\section{GREEK-ENGLISH VOCABULARY}

$\delta \varepsilon$, copulative and adversative (milder than $\dot{\alpha} \lambda \lambda \dot{\alpha}$ ) conj., postpositive, in the next place, and; but, on the other hand

$\delta \varepsilon i$, it is necessary

$\delta \varepsilon u ́ \tau \varepsilon \rho \circ \varsigma,-\alpha,-0 \nu$, second

$\delta \iota \dot{\alpha}$, prep., with gen., through, by; with acc., because of, on account of, for the sake of

$\delta \iota \alpha ́ \beta 0 \lambda \circ \varsigma,-0 \cup, \dot{0}$, devil

$\delta \iota \alpha \theta \dot{\eta} \times \eta,-\eta \varsigma, \dot{\eta}$, covenant, testament

$\delta$ ¿ $\alpha \times \circ \vee \varepsilon \dot{c} \omega$, I serve, minister

$\delta$ ¿laxovía, $-\alpha \varsigma, \dot{\eta}$, service, ministry

$\delta \_\alpha ́ x o$ vos, -ou, ó, servant, minister, deacon

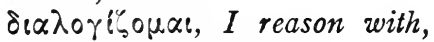
discuss, consider

$\delta\llcorner\alpha \dot{v} 0<\alpha,-\alpha \varsigma, \dot{r}$, mind, understanding

$\delta \iota \delta \dot{\alpha} \sigma \times \alpha \lambda \circ \varsigma,-0 u, \dot{\delta}$, teacher

$\delta\llcorner\delta \dot{\alpha} \sigma \times \omega, I$ teach

$\delta i \delta \omega \mu$, I give, deliver

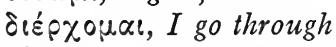

$\delta(x<105,-\alpha,-0 v$, righteous

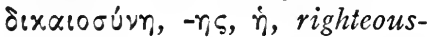
ness

óxaı́ów, I declare righteous, justify

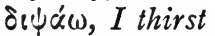

$\delta\llcorner\omega \dot{x} \omega$, I follow after, pursue, persecute

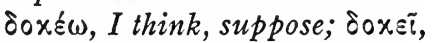
it seems good

$\delta \delta \xi \alpha,-\eta s, \dot{\eta}$, glory

$\delta \circ \xi \alpha^{\prime} \zeta \omega$, I glorify

$\delta \circ \cup \lambda \varepsilon \cup \cup \omega, I$ am a servant, $I$ serve

$\delta \circ \tilde{\lambda} \mathrm{O},-\circ \cup, \delta$, servant
Súvaual, I am able, can

$\delta u ́ v \alpha \mu l \varsigma,-\varepsilon \omega \varsigma, \dot{\eta}$, power

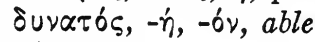

¿úo, two

$\delta \omega \delta \delta \varepsilon \times \alpha$, twelve

$\delta \tilde{\omega} \rho \circ \nu,-o u, \tau \delta$, gift

$\mathrm{E}$

$\varepsilon \dot{\varepsilon} \nu$, conditional particle, if

$\varepsilon \dot{\varepsilon} \alpha \mu \dot{n}$, with a substantive $=$ except, unless

$\dot{\varepsilon} \alpha \cup \tau \circ \tilde{u}, \quad-\tilde{\eta} \varsigma, \quad-\circ \tilde{u}, \quad$ (rarely

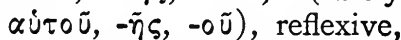
himself, herself, itself

" $\beta \alpha \lambda \circ \mathrm{\nu}$, I threw, cast; sec. aor. of $\beta \alpha \dot{\alpha} \lambda \omega$.

$\varepsilon \beta \eta \nu, I$ went; $\mu \iota$ - aorist of $\beta \alpha i v \omega$.

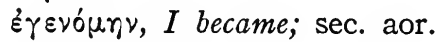
of $\gamma^{\prime}$ ivouas.

है $\gamma \nu \omega \nu, I$ knew; $\mu i$ - aorist of $\gamma(\nu \omega \dot{\sigma} \times \omega$.

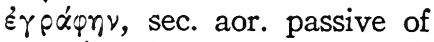

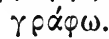

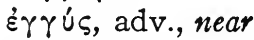

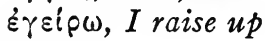

$\varepsilon \nvdash \hat{\omega}, I$

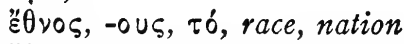

"ै $0 \circ \varsigma,-\circ \cup \varsigma, \tau \delta$, custom

$\varepsilon l$, conditional particle, if

el $\mu$, , with a substantive $=$ except, unless

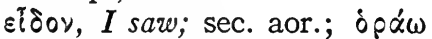
used in present

Eipi, I am

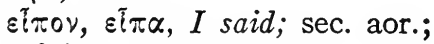
$\lambda \varepsilon \dot{\varepsilon} \gamma \omega$ used in present

sip $\eta_{\eta} \eta,-\eta s, \dot{\eta}$, peace

eis, prep., into, used only with the acc.

sic, $\mu\{\alpha$, हैv, one 


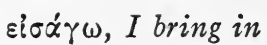

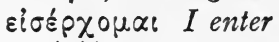
$\varepsilon \times(\xi \xi)$, prep., out, out of, from within, used only with the abl.

$\varepsilon \times \beta \alpha^{\prime} \lambda \lambda, \omega, I$ throw out, cast out $\varepsilon \times \varepsilon \bar{\imath}$, adv., there

Éxeivos, $-r_{i},-0$, demons. pron., that (one)

$\varepsilon \dot{x} \times \lambda \gamma_{r} \sigma i \alpha,-\alpha \varsigma, \dot{\eta}$, assembly, church

Éx

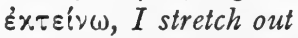

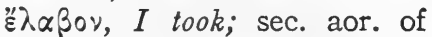
$\lambda \alpha \mu \beta \dot{\alpha} v \omega$.

हैं

$\varepsilon \lambda \varepsilon \dot{\theta} \theta \varepsilon \rho \circ \varsigma,-\alpha,-0 \nu$, free

$\varepsilon \lambda \pi \zeta_{\zeta}^{\prime \prime} \omega, I$ hope

$\varepsilon \lambda \pi i \varsigma,-i \delta \circ \varsigma, \dot{\eta}$, hope

हैं $\lambda \varepsilon i \pi \omega$.

$\varepsilon \dot{\varepsilon} \alpha \cup \tau 0 \bar{u},-\tilde{r}_{\zeta}$, , reflexive pron., myself

É $\mu \beta \alpha i v \omega, I$ go into, embark

$\varepsilon \dot{\varepsilon} \delta^{\prime} s,-\dot{\eta}$, óv, poss. pron., $m y$, mine

$\varepsilon$ 'v, prep., in; used only with the loc.

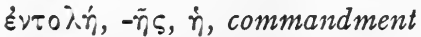

$\varepsilon \dot{\varepsilon} \omega \pi$ เoy, prep. with gen., before, in the presence of

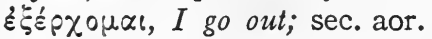
$\dot{\varepsilon} \xi \tilde{\eta} \lambda \theta 0 \%$.

"' $\xi \varepsilon \sigma \tau l y$, it is laweful, is possible

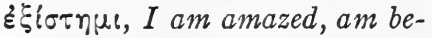
side myself

$\varepsilon \xi \xi \cup \cup \sigma^{\prime} \alpha,-\alpha \varsigma, \dot{\eta}$, authority, power

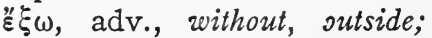
used with abl., without, outside

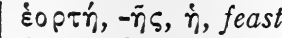

$\dot{\varepsilon} \pi \alpha \gamma \gamma \varepsilon \lambda \hat{\imath} \alpha,-\alpha \varsigma, \dot{\eta}$, promise

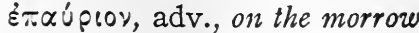

$\varepsilon \dot{\varepsilon} \varepsilon \rho \omega \tau \alpha \dot{\alpha} \omega, I$ question, ask (a question)

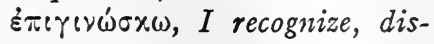
cover

$\varepsilon \pi \_\delta \delta \delta \omega \mu, I$ give over

$\varepsilon \pi: \theta \cup \mu\{\alpha,-\alpha \varsigma, \dot{\eta}$, desire

$\varepsilon \pi \operatorname{\varepsilon } \theta u \tilde{\varepsilon} \omega, I$ desire

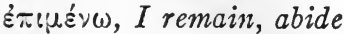

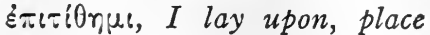
upon

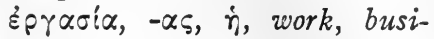
ness

Ëpyov, -ou, tó, work

हैpruos, -ou, $\dot{\eta}$, wilderness, desert

हैp\%oual, I go, come

$\varepsilon p \omega \tau \alpha \dot{\alpha} \omega, I$ ask (question)

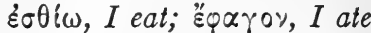

हैं\% $\alpha \tau 0 \varsigma,-\eta,-0 \%$, last

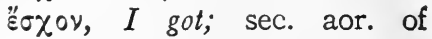
है $\chi \omega$.

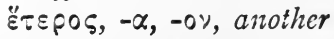

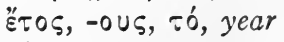

$\varepsilon \bar{j}$, adv., well

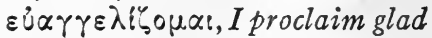
tidings (preach the gospel)

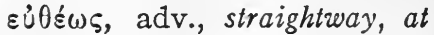
once

Eupiox., I find

sípov, I found; sec. aor. of Eupi $\sigma \% \omega$.

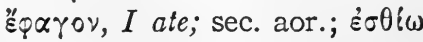
used in present

'Ę̣́́olos, -x, -0y, Ephesian Epirir,ul, I stand upon or by, come upon

ĚquYov, sec. aor. of $\rho \varepsilon u ́ \gamma \omega$.

" $\chi \omega$, I have, hold, get 


\section{GREEK-ENGLISH VOCABULARY}

\section{$\mathrm{Z}$}

$\zeta \alpha$ ' $\omega$, I live

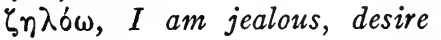
eagerly

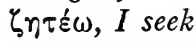

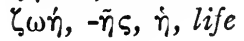

\section{$\mathrm{H}$}

ضै, conj., or

$\dot{\eta} \gamma \varepsilon \mu \omega \dot{\nu} \nu,-o ́ v o s, \delta$, leader, governor

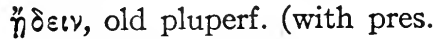
meaning) of $0 \tilde{i} \delta \alpha$.

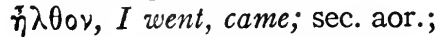

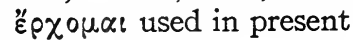

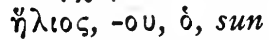

$\dot{\eta} \mu \varepsilon p \alpha,-\alpha \varsigma, \dot{\eta}$, day

$\dot{\eta} \mu \varepsilon \dot{\tau \varepsilon \rho \circ \varsigma, ~} \alpha,-\circ \nu$, poss. pron., our

\section{$\Theta$}

$\theta \dot{\alpha} \lambda \alpha \sigma \sigma \alpha,-\eta \varsigma, \dot{\eta}$, sea

$\theta \alpha \dot{v \alpha \tau o \varsigma, ~-0 \cup, \delta, ~ d e a t h ~}$

$\theta \alpha v \alpha \tau o ́ \omega, I$ put to death

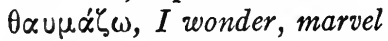

$\theta \varepsilon \alpha \dot{o} \mu \alpha \mathrm{l}, I$ behold, see, look at

$\theta \varepsilon \dot{\varepsilon} \eta \eta \mu \alpha,-\alpha \tau \circ \varsigma, \tau \delta$, will

$\theta \xi \lambda \omega, I$ wish, will

$\theta \varepsilon o ́ s,-o u \tilde{~ o ́, ~ G o d ~}$

$\theta \varepsilon \rho \propto \pi \varepsilon \dot{u} \omega$, I heal, doctor

$\theta \varepsilon \omega \rho \varepsilon \dot{\omega}$, I look at, gaze, see

$\theta \lambda(\dot{\imath} \iota \varsigma,-\varepsilon \omega \varsigma, \dot{\eta}$, tribulation, distress

$\theta \rho i \xi, \tau \rho \imath \% o ́ s, \dot{\eta}$, hair

Opóvos, -ou, o, throne

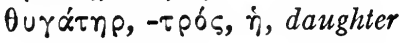

\section{I}

láoual, I heal

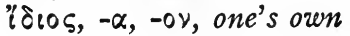

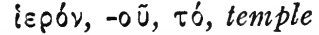

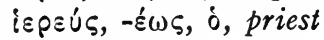

inue, I send

iv $\alpha$, conj. generally with subjunctive, in order that, that

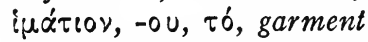

"ornue, I make to stand, place, stand

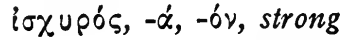

\section{$\mathrm{K}$}

$x \alpha \theta \alpha \rho \zeta_{\sigma}^{\prime} \omega, I$ purify

$x \propto \theta$ i $\sigma \tau \eta \mu$, , I set down, appoint xal, conj., and; also; even; $x \alpha i$...xai, both ... and $x \propto \times o ́ s,-\hat{n},-o ́ v$, evil, bad $x \propto \lambda \varepsilon^{\prime} \omega, I$ call

$x \propto \lambda \dot{\sigma} s,-\dot{\eta},-o v$, good, beautiful $x \propto \lambda \tilde{\omega} \varsigma$, adv., well, finely

$x \alpha \rho \delta\{\alpha,-\alpha \varsigma, \dot{\eta}$, heart

$x \alpha \rho \pi \delta s,-o \tilde{u}, \dot{\delta}$, fruit

$x \propto \tau \dot{\alpha}$, prep., with gen., down (upon), against; with abl., down (from); with acc., down (along), through, according to $x \propto \tau \alpha \beta \alpha i v \omega, I$ am going down, $x \propto \tau \alpha \lambda \cup \omega, I$ destroy

$x \alpha \tau \varepsilon \sigma \theta i \omega, I$ eat $u p$

$x \in i \mu \alpha \iota, I$ lie (am laid)

$x \varepsilon \varphi \alpha \lambda \dot{\eta},-\tilde{\eta} s, \dot{\eta}$, head

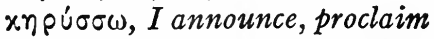
xolvós, -ń, -óv, common, unclean

xoevów, I make common, unclean

$x . \delta \pi \tau \omega, I$ beat, strike

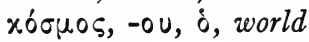

$x p \alpha \dot{\beta} \alpha \tau \tau$ os, -ou, $\delta$, pallet, bed $x \rho \alpha{ }^{\prime} \omega, I$ cry out

$x \rho\{\mu \alpha,-\alpha \tau \circ \varsigma, \tau \delta, j u d g m e n t$ 


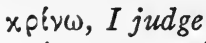

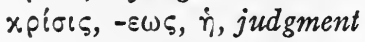

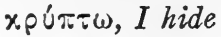

$x \tau$ icts, $-\varepsilon \omega \varsigma, \dot{\eta}$, creation

xupısíw, I am lord of, rule

(over) with gen.

xúpros, -ou, ó, Lord

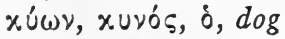

$x \omega \lambda \cup u \omega, I$ hinder

$x \dot{\omega} \mu \eta,-\eta \varsigma, \dot{\eta}$, village

\section{$\Lambda$}

$\lambda \alpha \lambda \varepsilon \dot{\varepsilon} \omega, I$ speak

$\lambda \alpha \mu \beta \alpha^{\prime} \nu \omega$, I take, receive; sec. aor., है $\lambda \alpha \beta \circ \nu$. $\lambda \alpha o_{s},-0 \tilde{u}, \dot{\delta}$, people

$\lambda \varepsilon \hat{\gamma} \omega$, I say, speak

$\lambda \varepsilon i \pi \omega, I$ leave, abandon; sec. aor., "ै $\lambda_{\imath}$ เ 0 v.

$\lambda \eta \sigma \tau \dot{\eta} \varsigma,-o \tilde{u}, \delta$, robber

$\lambda\{0 \circ 5,-\circ \cup, \delta$, stone

$\lambda\left(4, \lambda_{\bullet} \beta \sigma s, \delta\right.$, the $S . W$. wind

$\lambda \circ \gamma \circ 5,-0 u, \delta$, word

$\lambda \circ u$ w, $I$ wash

$\lambda \cup \pi \hat{\varepsilon} \omega, I$ grieve

$\lambda u ́ \omega$, I loose

\section{M}

$\mu \alpha \theta \eta \tau \eta \dot{s}$, , - ũ, $\dot{o}$, disciple

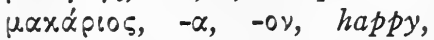
blessed

$\mu \tilde{\alpha} \lambda \lambda_{0} \%$, adv., more, rather

$\mu \alpha \nu \theta \dot{\alpha} \nu \omega$, I learn; sec. aor., है $\mu \alpha \theta 0 \nu$.

$\mu \alpha \rho \tau \cup \rho \varepsilon ́(\omega, I$ bear witness, testify

$\mu \dot{\alpha} \sigma \tau \iota \xi,-\iota \gamma \circ \varsigma, \dot{\eta}$, whip, scourge, plague

$\mu \dot{\alpha} \chi \alpha, p \alpha,-\alpha \varsigma, \dot{\eta}$, sword

$\mu \varepsilon^{\prime} \gamma \alpha \xi, \mu \varepsilon \gamma \alpha^{\prime} \lambda \eta, \mu \varepsilon^{\prime} \gamma \alpha$, great $\mu \varepsilon \lambda_{\lambda \varepsilon}$, it concerns, is a care, with dat.

$\mu \varepsilon \hat{\varepsilon} \lambda \omega \omega, I$ am about (or going) to do something

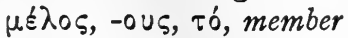

$\mu \varepsilon \dot{v} \omega$, I remain

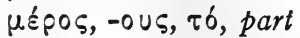

$\mu \varepsilon \tau \dot{\alpha}$, prep., with gen., with; with acc., after; $\mu \varepsilon \tau \dot{\alpha} \tau \alpha \tilde{u} \tau \alpha$, after these things, after this

$\mu \varepsilon \tau \alpha \beta \alpha i v \omega, I$ pass over, depart

$\mu \varepsilon \tau \alpha$ ขอย่ $\omega$, I repent

$\mu \dot{r}$, not

$\mu \gamma_{\gamma} \delta \varepsilon i \varsigma, \mu \eta_{\eta} \delta \varepsilon \mu i \alpha, \mu \eta \delta \varepsilon \varepsilon$, no one, nothing

$\mu \dot{r}_{i} \tau \varepsilon \ldots \mu \dot{\gamma} \tau \varepsilon$, neither ... nor

$\mu \hat{n} \tau \eta \rho,-\tau \rho o ́ s, \dot{\eta}$, mother

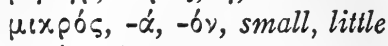

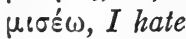

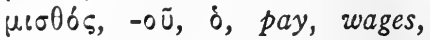
reward

$\mu \nu \tilde{\alpha}, \mu \nu \tilde{\alpha} \varsigma, \dot{\eta}$, mina (a weight and a sum of money)

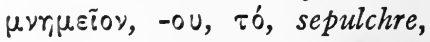
tomb

$\mu \nu$ r. gen.

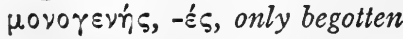

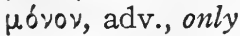

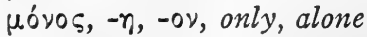

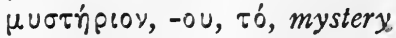

\section{$\mathrm{N}$}

vaós, -oũ, ó, temple

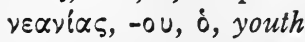

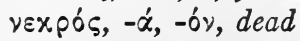

vย́os, $-\alpha,-0 \nu$, young, new

$\nu \varepsilon \varphi \hat{\varepsilon} \lambda \eta,-\eta \varsigma, \dot{\eta}$, cloud 


\section{GREEK-ENGLISH VOCABULARY}

$\nu \varepsilon \omega \times b \rho o s,-0 u, \delta$ or $\dot{\eta}$, templekeeper

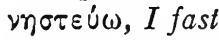

$\nu$ vx $\alpha \omega, I$ conquer

$\nu i \pi \tau \omega, I$ wash

vouiš $\omega, I$ think, suppose

vópos, -ov, i, law

ขŨข, adv., now

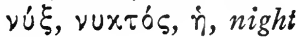

$\mathrm{O}$

$\dot{\delta}, \dot{\eta}, \tau \delta$, the definite article the

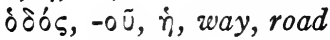

оi $\delta \alpha, I$ know

oixi $\alpha,-\alpha \varsigma, \dot{\eta}$, house

oixodopis $\omega$, I build

oixos, -ou, ó, house

olvos, -ou, o, wine

ठ $\lambda \dot{i}$ $\circ 5,-\eta,-0 \nu$, few, little, small

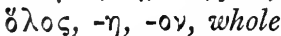

onoców, I make like

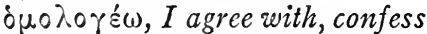

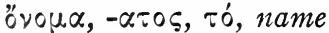

ö тоu, rel. adv., where

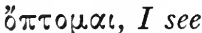

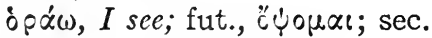
aor., Eiंठov

ö, r̆, ö, rel. pron., who, which, that, what

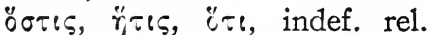
pron., who, which, that, what

ö $\tau \alpha \nu$, rel. temporal adv. used with the subj. and ind., whenever, when

ถ้ $\tau \varepsilon$, rel. temporal adv. used with the ind., when

ö $\tau$, conj., because, that

ou, not; oux before vowels; o ux before rough breathing ou $\mu$ '́, see page $I 73$

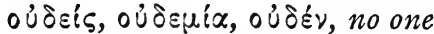

(nobody), nothing

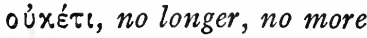

oúpavós, -oũ, ó, heaven

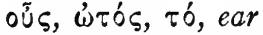

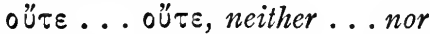

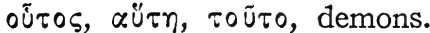

pron., this (one)

b $\varphi \varepsilon\{\lambda \omega, I$ owe, ought; sec. aor.

without augment, "̋ $\varphi \varepsilon \lambda \circ \nu$

o $\phi \theta \alpha \lambda \mu \sigma_{\varsigma},-0 \tilde{u}, \dot{o}$, eye

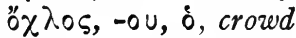

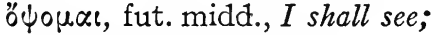
opá $\omega$ used in present

\section{$\Pi$}

$\pi \alpha\llcorner\delta i o v,-o v, \tau \delta$, little child

$\pi \alpha \lambda \alpha เ o ́ \varsigma,-\alpha$, -óv, old, ancient

$\pi \dot{\alpha} \lambda เ \nu$, adv., again

$\pi \alpha \rho \alpha$, prep., with the loc., by

or at (the side of); with the

abl., from (the side of); with

the acc., along (side of)

$\pi \alpha p \alpha \beta \circ \lambda \dot{\eta},-\tilde{n} s, \dot{\eta}$, parable

$\pi \alpha \rho \alpha \delta i \delta \omega \mu l, I$ give over (to

another), deliver up, betray

$\pi \alpha p \alpha \times \alpha \lambda \varepsilon \omega, I$ beseech, exhort,

encourage

$\pi \alpha \rho \alpha \dot{x} \lambda \eta \nabla \iota s,-\varepsilon \omega \varsigma, \dot{\eta}$, exhortation

$\pi \alpha p \alpha \lambda \alpha \mu \beta \alpha \dot{\nu} \omega$, I take, receive;

sec. aor., $\pi \alpha \rho \varepsilon \lambda \alpha \beta \circ \nu$

$\pi \alpha p \alpha \tau i \theta \eta \mu \iota, I$ set before, commit

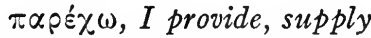

$\pi \alpha \rho^{\prime \sigma \sigma \eta \eta \mu t, ~ I ~ p l a c e ~ b e s i d e, ~ s t a n d ~}$ by

$\pi \alpha \rho \circ \cup \sigma i \alpha,-\alpha \varsigma, \dot{\eta}$, coming, presence

$\pi \tilde{\alpha} \varsigma, \pi \tilde{\alpha} \sigma \alpha, \pi \tilde{\alpha} \nu$, all, every 
$\pi \dot{\alpha} \sigma \alpha \alpha$, (indeclinable), $\tau$ ó, the Passover

$\pi \dot{\alpha} \sigma \chi \omega, I$ suffer; sec. aor., हैं $\alpha \theta 0 \nu$

$\pi \alpha \tau \dot{n} \rho,-\tau \rho \dot{s} s, \dot{\delta}$, father

$\pi \alpha u ́ \omega$, I stop; midd., $\pi \alpha \dot{u} \circ \mu \alpha \iota$, I cease

$\pi \varepsilon i \theta \omega, I$ persuade

$\pi \varepsilon เ \nu \alpha$ á, I hunger, am hungry

$\pi \varepsilon เ \rho \alpha \zeta^{\prime} \omega$, I test, tempt

$\pi \varepsilon \dot{\varepsilon} \mu \omega \omega, I$ send

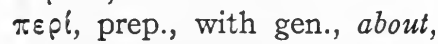

concerning; with abl., from

around; with acc., round

about, about, concerning

$\pi \varepsilon p\llcorner\alpha \dot{\gamma} \gamma \omega$, I go about, carry about

$\pi \varepsilon p(\pi \alpha \tau \varepsilon \dot{\varepsilon} \omega$, I walk, live

$\pi \varepsilon p r \sigma o o ́ s,-\dot{\eta},-\delta v$, abundant

$\pi เ \sigma \tau \varepsilon \dot{\omega} \omega, I$ believe

$\pi i \sigma \tau \iota \varsigma,-\varepsilon \omega \varsigma, \dot{\eta}$, faith

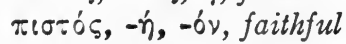

$\pi \lambda \alpha v \alpha \dot{\omega} \omega, I$ cause to wander (to err), lead astray

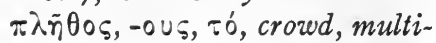
tude

$\pi \lambda \eta p o ́ \omega$, I make full, fill

$\pi \lambda$ noiov, adv., near; $\delta$ iो roio\%, neighbor

$\pi \lambda$ तoiov, -ou, $\tau$ ó, boat

$\pi \lambda \circ \operatorname{ú\sigma los},-\alpha$, - $0 \nu$, rich

$\pi \nu \varepsilon \tilde{u} \mu \alpha,-\alpha \tau \circ \varsigma, \tau o ́$, spirit

$\pi \delta \theta \varepsilon v$, interrog. adv., whence

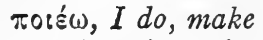

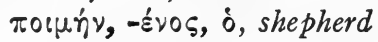

$\pi \dot{\gamma} \lambda เ \varsigma,-\varepsilon \omega \varsigma, \dot{\eta}$, city

$\pi 0 \lambda \dot{u}$, adv., much

$\pi 0 \lambda \dot{u} s, \pi 0 \lambda \lambda \dot{\eta}, \pi 0 \lambda \dot{u}$, much, many

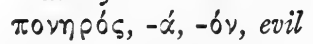

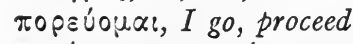

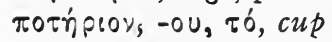

Toũ, interrog. adv., where

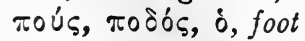
$\pi \rho \varepsilon \sigma \beta u ́ \tau \varepsilon \rho \circ \varsigma,-\circ u, \dot{o}$, elder $\pi$ piv ( $\ddot{\eta})$, before; see page I9o $\pi \rho \dot{0}$, prep. with abl., before $\pi \rho \circ \alpha \gamma \omega$, I lead forth, go before $\pi$ pó $\alpha \tau 0 \nu,-0 u, \tau o ́$, sheep $\pi$ ós, prep., with loc., near, by; with acc., towards, to; with abl. (once), "from the point of view of"

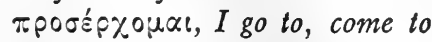

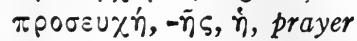
$\pi \rho \circ \sigma \tau i \theta r, \mu, I$ add, give in addi. tion $\pi \rho \delta \sigma \omega \pi 0 \nu,-o u, \tau o ́$, face

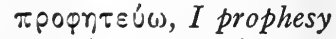

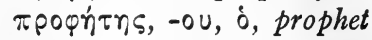
$\pi p \tilde{\omega} \tau \circ \varsigma_{,}, \eta,-0 \nu$, first $\pi \omega \pi 0 \tau \varepsilon, \mathrm{adv}$, ever yet $\pi \tilde{\omega} s, \mathrm{adv} .$, how

\section{$\mathrm{P}$}

pंr $\mu \alpha,-\alpha \tau \circ \varsigma, \tau \delta$, word

$\Sigma$

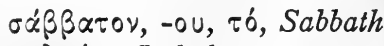
$\sigma \alpha \lambda \varepsilon \cup \dot{ } \omega$, I shake

$\sigma \alpha \dot{\lambda} \pi เ \gamma \xi,-\imath \gamma \gamma \circ \varsigma, \dot{\eta}$, trumpet $\sigma \alpha \dot{\rho} \xi$, $\sigma \alpha \rho x o ́ \varsigma, \dot{\eta}$, flesh

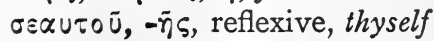

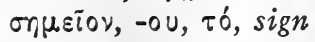
ớuєpov, adv., to-day, this day

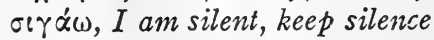
$\sigma \omega \omega \alpha \dot{\alpha}, I$ am silent, keep silence

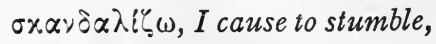
offend

oxrvów, I dwell (as in a tent) 
oxó $\lambda \circ \psi,-0 \pi \circ \varsigma$, o stake, thorn

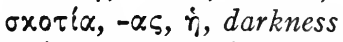

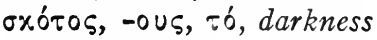

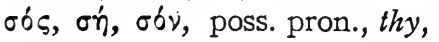
thine

$\sigma o \varphi i \alpha,-\alpha . s, \dot{r}$, wisdom ooọós, -ŕn, -óv, wise

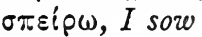
$\sigma \pi \dot{\varepsilon} p \mu \alpha,-\alpha \tau 0 \varsigma, \tau o ́$, seed

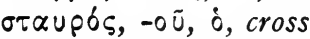

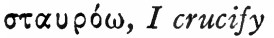
$\sigma \tau \varepsilon \dot{\lambda} \lambda \omega, I$ send $\sigma \tau \varepsilon \nu \alpha^{\prime \prime} \omega, I$ groan

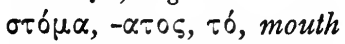

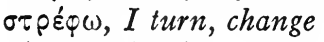
oú, thou (you) ou'v, prep., with, used only with the instrumental

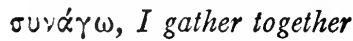

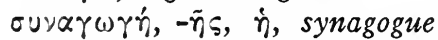

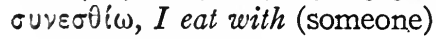
ouvirul, I perceive

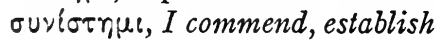
oúpw, I drag, draw $\sigma \omega, \zeta \omega, I$ save $\sigma \tilde{\omega} \mu \alpha,-\alpha \tau \circ \varsigma, \tau \delta$, body

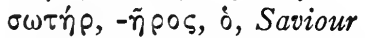

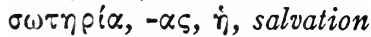

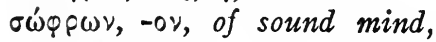
sober-minded

$\mathrm{T}$

$\tau \alpha \pi \varepsilon เ v o ́ \omega$, I make low, humble $\tau \alpha \chi \varepsilon \dot{\varepsilon} \omega \varsigma$, adv., quickly

$\tau \alpha \varkappa \dot{y}$, adv., quickly

$\tau \grave{\varepsilon}$. . . x $\alpha i$, both . . . and

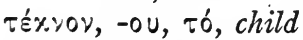

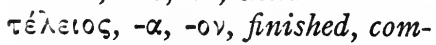
plete

$\tau \varepsilon \lambda \varepsilon เ o ́ \omega, I$ end, complete, fulfill $\tau \varepsilon \lambda \varepsilon \cup \tau \dot{\alpha} \omega$, (I finish), I die $\tau \varepsilon \lambda \varepsilon \dot{ } \omega, I$ finish, end, complete

$\tau \varepsilon^{\prime} \lambda \circ \varsigma,-\circ \cup \varsigma, \tau o ́$, end

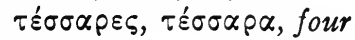

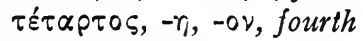

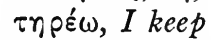

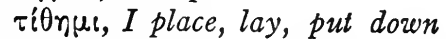

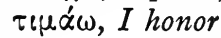

$\tau i \varsigma, \tau i$, interrog. pron., who, which, what

$\tau \iota \varsigma, \tau$, indef. pron., one, a certain one, a certain thing; some one, something

$\tau 0 \lambda \mu \alpha \omega, I$ dare

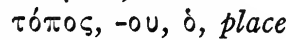

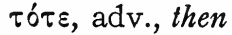

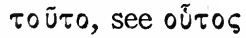

$\tau p \varepsilon i \varsigma, \tau p i \alpha$, three

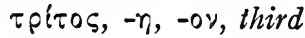

$\tau \cup \varphi \lambda \sigma_{s},-\dot{\eta}$, -óv, blind

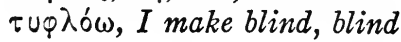

\section{$\Upsilon$}

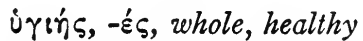



viós, -oũ, $\dot{0}$, son

¿ $\mu \varepsilon \varepsilon \varepsilon \rho \circ \varsigma,-\alpha,-0 \nu$, poss. pron., your

ن $\pi \dot{\gamma} \gamma \omega$, I go away, depart

uं $\varepsilon p$, prep., with abl., in behalf of, in the interest of; instead of; in place of; for the sake of; about, concerning; with acc., over, above, beyond

iтó, prep., with abl., by; with acc., under

نтoxpitńs, -оj, j, pretender, hypocrite 
$\Phi$

Qqivw, I shine, appear

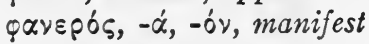
qavepó $\omega$, I make manifest

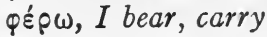
$\varphi \varepsilon u ́ \gamma \omega$, I flee, take flight; sec. aor., हैquro:

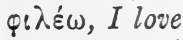

ólios, -ou, ó, friend coßśou, al, I am afraid, I fear óó̧०द, -ou, ó, fear

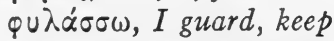
 $\varphi \omega \nu \dot{\eta}_{1},-\tilde{\eta} \varsigma, \dot{\eta}$, voice

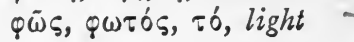

$\mathrm{X}$

$\chi \alpha i \rho \omega, I$ rejoice $\chi \alpha \rho \alpha \dot{\alpha},-\tilde{\alpha} s, \dot{\eta}, j o y$ $\chi \dot{\alpha} \rho \bullet \varsigma,-\imath \tau \circ \xi, \dot{\eta}$, grace $\chi \alpha \dot{\alpha}\llcorner\sigma \mu \alpha,-\alpha \tau \circ \varsigma, \tau \delta$, gift, free gift

$\chi \varepsilon l \rho, \chi \varepsilon\llcorner\rho \delta ́ s, \dot{\eta}$, hand

$\chi p \varepsilon i \alpha,-\alpha s, \dot{\eta}$, need

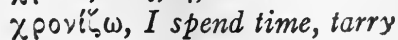

xpóvos, -ou, ó, time

$\Psi$

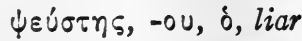

$\dot{\varphi} \cup \chi_{\dot{\eta}},-\tilde{\eta} s, \dot{\eta}$, soul

\section{$\Omega$}

$\omega \delta \varepsilon$, adv., here, hither

$\ddot{\omega} p \alpha,-\alpha \varsigma, \dot{\eta}$, hour

is, rel., comp., and temporal adv., as, when

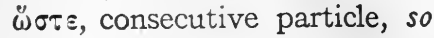
that

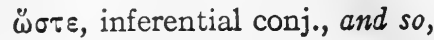
therefore 


\section{ENGLISH-GREEK VOCABULARY}

A

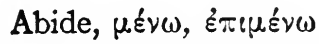

After these things, $\mu \varepsilon \tau \dot{\alpha} \tau \alpha \tilde{v} \tau \alpha$ Am, $\varepsilon i \mu i$

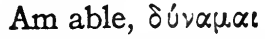

Am about (to do something). $\mu \varepsilon^{\prime} \lambda \lambda \omega$

And, $x \alpha$ l

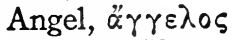

All, $\pi \tilde{\alpha} \varsigma$, , $\lambda \circ \varsigma^{\circ}$

Announce, xฤpúrow

Answer, $\dot{\alpha} \pi 0 x \rho i v o \mu \alpha$ i

Apostle, $\dot{\alpha} \pi \dot{\sigma} \sigma 0 \lambda \circ \varsigma$

Appoint, $x \alpha \theta i \sigma \tau \eta \mu \iota$

Ask (a question), है $\rho \omega \tau \alpha \dot{\alpha} \omega$

\section{B}

\section{Bad, xaxbs}

Baptize, $\beta \alpha \pi \tau i \zeta \omega$

Bear witness, $\mu \alpha \rho \tau \cup \rho \varepsilon ́ \omega$

Because (conj.), ö $\tau \imath$

Become, $\gamma^{\text {ivouat }}$

Before, $\pi$ pb with abl.

Beget, $\gamma \varepsilon \nu \nu \alpha ́ \omega$

Beginning, $\dot{\rho} \rho \times \dot{n}$

Behold, $\beta \lambda \varepsilon \pi \omega$

Believe, $\pi \iota \sigma \tau \varepsilon \dot{\omega} \omega$

Blind (adj.), $\tau \cup \varphi \lambda b s$

Blind (verb), $\tau \cup \varphi \lambda \sigma \omega$

Boat, $\pi$ तoiov

Bread, äpros

Brother, $\alpha \delta \varepsilon \lambda \varphi b \varsigma$
But, $\alpha \lambda \lambda \dot{\alpha}$

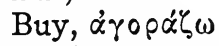

By (denoting agent), i $\pi \delta$ witk $a b l$.

- $\mathrm{C}$

Call, $x \propto \lambda \varepsilon \hat{\varepsilon} \omega$

Came to pass, है $\gamma \varepsilon v \varepsilon \tau o$

Care, it is a, $\mu \varepsilon \varepsilon_{\lambda \varepsilon \ell}$

Cast out, Éx $\beta \dot{\alpha} \lambda \lambda \omega$

Cease, $\pi \alpha \dot{0} 0 \mu \alpha \iota$

Certain (indef.), $\tau \iota \varsigma, \tau \iota$

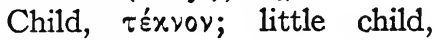
$\pi \alpha i \delta i o v$

Christ, Xplorbs

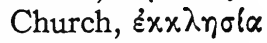

Come, "̈pxoual

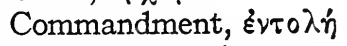

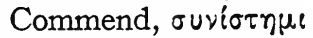

Concerning, $\pi \varepsilon \rho l$ with gem

Covenant, $\delta\llcorner\alpha \theta \dot{\eta} \times \eta$

Crowd, oै $\chi$ 入os

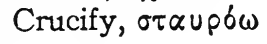

\section{D}

Darkness, $\sigma \times 0 \tau\{\alpha, \sigma \times 6 \tau \circ \varsigma$

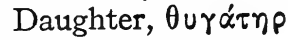

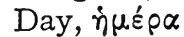

Dead, vexpós

Defile, xouvbo

Demon, satubviov

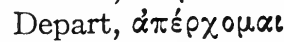

Destroy, $x \alpha \tau \alpha \lambda u ́ \omega$ 
Devil, $\delta\llcorner\alpha \dot{\beta} 0 \lambda \circ \varsigma$

Die, $\alpha \pi 0 \theta \nu \dot{\sigma} \sigma x \omega$

Disciple, $\mu \alpha \theta \eta \tau \dot{\eta} \varsigma$

Do, $\pi 0 เ \varepsilon ́ \omega$

\section{$\mathrm{E}$}

Eat, $\varepsilon^{\circ} \sigma 0 i \omega$

Eat up, $x \alpha \tau \varepsilon \sigma \theta i \omega$

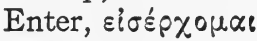

Eternal, aióvios

Every, $\pi \tilde{\alpha} \varsigma$

Evil, xaxós

Eye, $\delta \varphi \theta \alpha \lambda \mu \delta s$

\section{F}

Face, $\pi \rho \delta \sigma \omega \pi 0 \nu$

Faith, $\pi i \sigma \tau l \varsigma$

Faithful, $\pi เ \sigma \tau \delta \varsigma$

Father, $\pi \alpha$ rinp

Fear, oßśou

Find, eu pioxw

First, $\pi \rho \tilde{\omega} \tau 0 \varsigma$

Follow, $\alpha \times 0 \lambda 0 u \theta \dot{\varepsilon} \omega$

Foot, $\pi 0$ ús

For (conj.), $\gamma \alpha \dot{p} \rho$

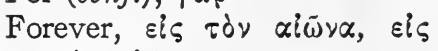

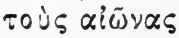

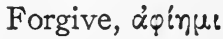

Friend, $\varphi$ i $\lambda \circ 5$

From, $\alpha$ đó with abl., $\pi \alpha p \alpha$ with $a b l$.

Fulfill, $\pi \lambda$ npów

\section{G}

Garment, i $\mu \dot{\alpha} \tau i 0 \nu$

Give, $\delta i \delta \omega \mu t$

Glorify, $\delta \circ \xi \dot{\alpha} \zeta_{\omega} \omega$

Glory, $\delta \delta \xi \alpha$

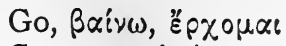

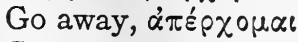

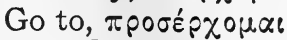

God, $\Theta \varepsilon o ́ s$

Good, $\alpha \gamma \alpha \theta \sigma s, x \alpha \lambda \sigma \sigma$

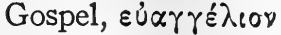

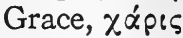

Great, $\mu \varepsilon \hat{\varepsilon} \gamma \alpha \varsigma$

Guard, $\varphi \cup \lambda \alpha ́ \sigma \sigma \omega$

$\mathrm{H}$

Hand, $\chi$ eip

Hate, $\mu\llcorner\sigma \varepsilon ́ \omega$

Have, "ै $\chi \omega$

$\mathrm{He}$ (in the oblique cases), aũós

$\mathrm{He}$ himself (intensive), aưrós

Heal, $\theta \varepsilon \rho \alpha \pi \varepsilon u ́ \omega$

Hear, $\alpha \times 0 \dot{\omega} \omega$

Heart, $x \alpha \rho \delta i \alpha$

Heaven, oupavós

Himself, herself (reflexive), $\dot{\varepsilon} \alpha \cup \tau \circ \tilde{u}, \dot{\varepsilon} \alpha \cup \tau \tilde{\eta} \varsigma$

Holy, ärios

Hope, $\dot{\varepsilon} \lambda \pi i \varsigma$

Hope (verb), हैं $\lambda i \zeta \omega$

Hour, "̈pa

House, oixos, oixi $\alpha$

How, $\pi \tilde{\omega} \varsigma$

\section{I}

I, हैं $\dot{\omega}$

If, si with the ind., है') with the subj.

In, év with loc.

In order that, iv $\alpha$ with subj.

Into, sis with acc.

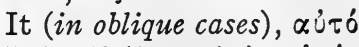

It itself (intensive), aن่ $\tau \dot{0}$

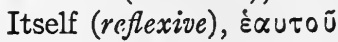




\section{ENGLISH-GREEK VOCABULARY}

$\mathrm{J}$

Joy, $\chi \alpha \rho \alpha$

Judge, $x p i v \omega$

Judgment, xpiols

Justify, $\delta เ x \alpha \imath o \omega$

K

Keep, $\tau \eta \rho \varepsilon ́ \omega$

Kill, $\dot{\alpha} \pi 0 x \tau \varepsilon i v \omega$

King, $\beta \alpha \sigma i \lambda \varepsilon \dot{v} \varsigma$

Kingdom, $\beta \alpha \sigma i \lambda \varepsilon i \alpha$

Know, $\gamma(v \dot{\omega} \sigma \times \omega$, ol $\delta \alpha$

\section{$\mathrm{L}$}

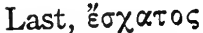

Law, vópos

Lawful, is, है $\xi \varepsilon \sigma \tau !$

Lay, $\tau(\theta \eta \mu \mathrm{e}$

Lay down, $\tau\{\theta \eta \mu$ s

Lead, $\ddot{\alpha} \gamma \omega$

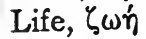

Light, $\varphi \tilde{\omega} \varsigma$

Look, $\beta \lambda \varepsilon \pi \omega$

Look up, $\alpha$ ข $\alpha \beta \lambda \varepsilon \dot{\varepsilon} \pi \omega$

Lord, xúplos

Love, $\dot{\alpha} \gamma \dot{\alpha} \pi \eta$

Love (verb), $\alpha \gamma \alpha \pi \alpha \dot{\alpha} \omega$

\section{M}

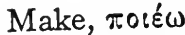

Man, $\ddot{\alpha} \nu \theta \rho \omega \pi 0 \varsigma$

Manifest, make, $\varphi \alpha v \varepsilon p b \omega$

Many, $\pi 0 \lambda u ́ s$

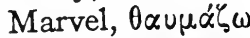

Master, xúplos

Mercy, है

Month, $\sigma \tau \delta \mu \alpha$
Mother, $\mu \dot{n} \tau \eta p$

Multitude, $\pi \lambda \tilde{n} \theta 0 \varsigma$

Myself (reflexive, in oblique

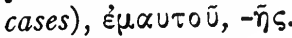

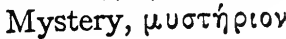

\section{$\mathrm{N}$}

Name, oैvoux

Nation, है $\theta$ v०s

Necessary, is, $\delta \varepsilon \tilde{\varepsilon}$

New, xaเvós, ขย์os

Night, $\nu u ̛ \xi$

No one, oujsis

Not, ou, oux, oux; $\mu$ in with subj., inf., and partic.

Nothing, oủ $\delta \varepsilon^{2}$

Now, ขŨ

\section{$\mathrm{O}$}

Obtain, है $\chi \omega^{-}$

On, हैे with loc., ह่ $\pi$ l with loc.

One another, $\alpha \lambda \lambda \dot{\eta} \lambda \omega \nu$

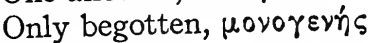

Other, $\ddot{\alpha} \lambda \lambda \circ \varsigma$

Out of, ह'x with abl.

$\mathrm{P}$

Parable, $\pi \alpha \rho \alpha \beta 0 \lambda \eta \dot{~}$

Part, $\mu$ épos

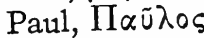

Peace, sipńvn

People, $\lambda \alpha$ ós

Persuade, $\pi \varepsilon^{i} \theta \omega$

Place, ró $\pi \circ s$

Place upon, ह่ $\pi \iota \tau\{\theta \eta \mu \iota$

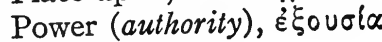

Power, súvauls

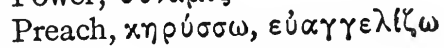


Priest, iєpєús

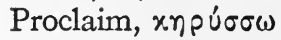

Promise, ह่ $\pi \alpha \gamma \gamma \varepsilon \lambda \hat{\imath} \alpha$

Prophet, $\pi$ popríns (5t)

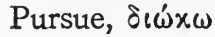

\section{Q}

Quickly, $\tau \alpha \chi \varepsilon ́ \omega \varsigma, \tau \alpha ́ \chi \alpha$

\section{$\mathbf{R}$}

Raise, है $\gamma \varepsilon i \rho \omega$

Rather, $\mu \tilde{\alpha} \lambda \lambda \circ \nu$

Receive, $\lambda \alpha \mu \beta \alpha \dot{\nu} \omega$

Remain, $\mu \varepsilon ́ v \omega$

Righteous, $\delta i x \alpha 10 s$

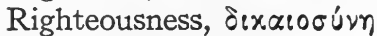

Ruler, $\ddot{\rho} \rho \chi \omega \nu$

\section{S}

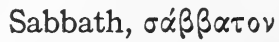

Salute, $\dot{\alpha} \sigma \pi \dot{\alpha} \zeta o \mu \alpha \iota$

Same, aưós with the article

Sanctify, $\dot{\alpha} \gamma \prec \dot{\alpha} \zeta \omega$

Save, $\sigma \omega^{\prime} \prime \omega$

Saviour, $\sigma \omega \tau \dot{p} p$

Scribe, $\gamma \rho \alpha \mu \mu \alpha \tau \varepsilon u ́ s$

Scripture, $\gamma \rho \alpha \varphi \eta ́ n$

Sea, $\theta \dot{\alpha} \lambda \alpha \sigma \sigma \alpha$

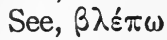

Seed, $\sigma \pi \varepsilon \hat{\varepsilon} p \mu \alpha$

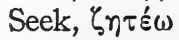

Send, $\pi \dot{\varepsilon} \mu \pi \omega, \sigma \tau \dot{\varepsilon} \lambda \lambda \omega$

Send forth, $\alpha \pi 0 \sigma \tau \varepsilon \hat{\varepsilon} \lambda \omega \omega$

Servant, $\delta \circ \tilde{u} \lambda \circ 5$

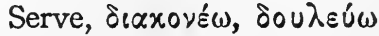

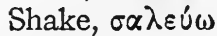

She (in the oblique cases), aúrós
She herself (intensive), aủrós

Sheep, $\pi$ pó $\beta \alpha \tau$

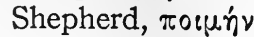

Sin, $\dot{\alpha} \mu \alpha \rho \tau i \alpha$

So that, $\ddot{\omega} \sigma \tau \varepsilon$

Son, viós

Soul, $\cup \times \times \dot{n}$

Sow, $\sigma \pi \varepsilon i \rho \omega$

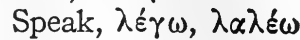

Spirit, $\pi \nu \varepsilon \tilde{u} \mu \alpha$

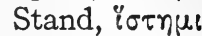

Stone, $\lambda: \theta 0 s$

Strong, ioxupos

Sword, $\mu \alpha^{\prime} \chi \alpha \iota p \alpha$

Synagogue, $\sigma u \nu \alpha \gamma \omega \gamma \dot{\eta}$

$\mathrm{T}$

Take, $\lambda \alpha \mu \beta \alpha \dot{\nu} \omega$

Take away, $\alpha$ l’ $\omega, \alpha \pi \alpha ! \rho \omega$

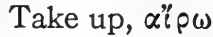

Teach, $\delta \iota \delta \dot{\alpha} \sigma \times \omega$

Teacher, $\delta เ \delta \dot{\alpha} \sigma x \alpha \lambda \circ \varsigma$

Temple, iєpóv

Testament, $\delta \prec \alpha \theta \dot{\eta} \times \eta$

Than, $\ddot{\eta}$

That, Éxeivos

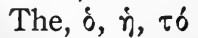

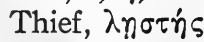

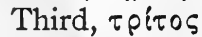

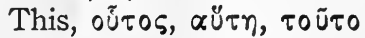

Thou, oú

Throne, $\theta$ póvos

Through, $\delta\llcorner\dot{\alpha}$ with genitive

Throw, $\beta \dot{\alpha} \lambda \lambda \omega$

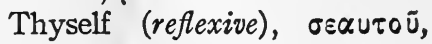
$-\tilde{n} s$

Touch, $\ddot{\alpha} \pi \tau \mu \alpha \iota$

Tribulation, $\theta \lambda i$ i 15

Truth, $\alpha \lambda \dot{\gamma} \theta \varepsilon \iota \alpha$

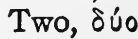


U

Under, ijob with acc.

V

Village, $x \omega \dot{\omega} \mu \eta$

Voice, $\varphi \omega \nu \dot{n}$

W

Walk, $\pi \varepsilon \rho \iota \pi \alpha \tau \varepsilon \dot{\varepsilon} \omega$

Wash, $\lambda \circ u^{\prime} \omega$, vi $\pi \tau \omega$

Way, óós

We, $\eta \dot{\mu} \mu \tilde{i} s$

Well, $x \alpha \lambda \omega \tilde{\omega}, \varepsilon \tilde{u}$

What, see Who

When, $\check{\tau} \tau \nu$, ö $\tau \varepsilon$

Where, rou
Who, which, that, what (relative), ös, "̈, ö

Who, which, what? (interrog.), $\tau i \varsigma, \tau i$

Whole, ö $\lambda \circ 5$

Why, $\tau i$

Will, $\theta \varepsilon \dot{\lambda} \eta \eta \mu \alpha$

Wisdom, oop̣ia

Wish, $\theta \dot{\varepsilon} \lambda \omega$

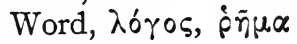

Work, हैp pov

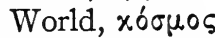

Worthy, $\ddot{\xi} \xi 05$

Write, $\gamma \rho \alpha \dot{\alpha} \omega$

Y

You (sing.), бú

You (plu.), úpeis 
Infinition((ev) Nuddelata) re a nume jg. $211 \$ 132$ 

mparcosiopg. $178-$ 


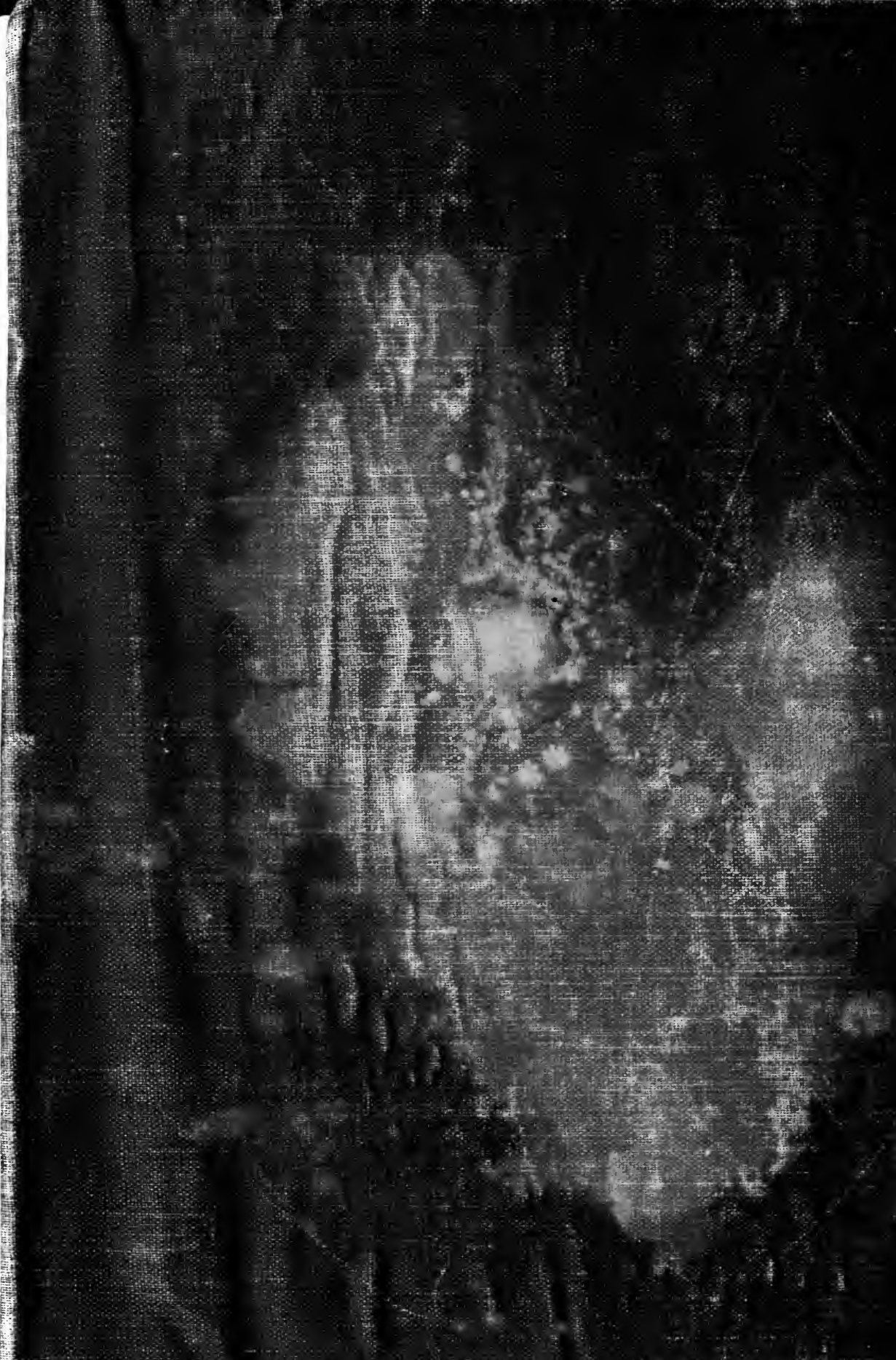

45 